\title{
BTS guideline for emergency oxygen use in adult patients
}

\author{
B R O'Driscoll, ${ }^{1}$ L S Howard, ${ }^{2}$ A G Davison ${ }^{3}$ on behalf of the British Thoracic Society
}

${ }^{1}$ Department of Respiratory Medicine, Salford Royal University Hospital, Salford, UK; ${ }^{2}$ Hammersmith Hospital, Imperial College Healthcare NHS Trust, London, UK; ${ }^{3}$ Southend University Hospital, Westcliff on Sea, Essex, UK

Correspondence to:

Dr B R O'Driscoll, Department of Respiratory Medicine, Salford Royal University Hospital, Stott Lane, Salford M6 8HD, UK; ronan.o'driscoll@srft.nhs.uk

Received 11 June 2008 Accepted 11 June 2008

\section{EXECUTIVE SUMMARY OF THE GUIDELINE}

Philosophy of the guideline

- Oxygen is a treatment for hypoxaemia, not breathlessness. (Oxygen has not been shown to have any effect on the sensation of breathlessness in non-hypoxaemic patients.)

- The essence of this guideline can be summarised simply as a requirement for oxygen to be prescribed according to a target saturation range and for those who administer oxygen therapy to monitor the patient and keep within the target saturation range.

- The guideline suggests aiming to achieve normal or near-normal oxygen saturation for all acutely ill patients apart from those at risk of hypercapnic respiratory failure or those receiving terminal palliative care.

\section{Assessing patients}

- For critically ill patients, high concentration oxygen should be administered immediately (table 1 and fig 1) and this should be recorded afterwards in the patient's health record.

- Oxygen saturation, "the fifth vital sign", should be checked by pulse oximetry in all breathless and acutely ill patients (supplemented by blood gases when necessary) and the inspired oxygen concentration should be recorded on the observation chart with the oximetry result. (The other vital signs are pulse, blood pressure, temperature and respiratory rate).

- Pulse oximetry must be available in all locations where emergency oxygen is used.

- All critically ill patients should be assessed and monitored using a recognised physiological track and trigger system.

\section{Oxygen prescription}

- Oxygen should be prescribed to achieve a target saturation of $94-98 \%$ for most acutely ill patients or $88-92 \%$ for those at risk of hypercapnic respiratory failure (tables 1-3).

- The target saturation should be written (or ringed) on the drug chart (guidance in fig 1).

\section{Oxygen administration}

- Oxygen should be administered by staff who are trained in oxygen administration.

- These staff should use appropriate devices and flow rates in order to achieve the target saturation range (fig 2).
Monitoring and maintenance of target saturation

- Oxygen saturation and delivery system should be recorded on the patient's monitoring chart alongside the oximetry result.

- Oxygen delivery devices and flow rates should be adjusted to keep the oxygen saturation in the target range.

- Oxygen should be signed for on the drug chart on each drug round.

\section{Weaning and discontinuation of oxygen therapy}

- Oxygen should be reduced in stable patients with satisfactory oxygen saturation.

- Oxygen should be crossed off the drug chart once oxygen is discontinued.

Oxygen is one of the most widely used drugs and is used across the whole range of specialities. The Guideline Group recognises that many clinicians will initially wish to read an abbreviated version of this guideline which is available to download from the BTS website (www.brit-thoracic.org.uk).

\section{SUMMARY OF KEY RECOMMENDATIONS FOR EMERGENCY OXYGEN USE}

Achieving desirable oxygen saturation ranges in acute illness (sections 6.7 and 6.8)

1. This guideline recommends aiming to achieve a normal or near-normal oxygen saturation for all acutely ill patients apart from those at risk of hypercapnic respiratory failure. [Grade D]

2. The recommended target saturation range for acutely ill patients not at risk of hypercapnic respiratory failure is $94-98 \%$. Some normal subjects, especially people aged $>70$ years, may have oxygen saturation measurements below $94 \%$ and do not require oxygen therapy when clinically stable. [Grade D]

3. Most non-hypoxaemic breathless patients do not benefit from oxygen therapy, but a sudden reduction of more than $3 \%$ in a patient's oxygen saturation within the target saturation range should prompt fuller assessment of the patient (and the oximeter signal) because this may be the first evidence of an acute illness. [Grade D]

4. For most patients with known chronic obstructive pulmonary disease (COPD) or other known risk factors for hypercapnic respiratory failure (eg, morbid obesity, chest wall deformities or neuromuscular disorders), a target saturation range of $88-92 \%$ is suggested pending the availability of blood gas results. [Grade $\mathbf{C}$ ] 
5. Some patients with COPD and other conditions are vulnerable to repeated episodes of hypercapnic respiratory failure. In these cases it is recommended that treatment should be based on the results of previous blood gas estimations during acute exacerbations because hypercapnic respiratory failure can occur even if the saturation is below $88 \%$. For patients with prior hypercapnic failure (requiring non-invasive ventilation or intermittent positive pressure ventilation) who do not have an alert card, it is recommended that treatment should be commenced using a $28 \%$ Venturi mask at $4 \mathrm{l} / \mathrm{min}$ in prehospital care or a $24 \%$ Venturi mask at $2-4 \mathrm{l} / \mathrm{min}$ in hospital settings with an initial target saturation of $88-92 \%$ pending urgent blood gas results. These patients should be treated as a high priority by emergency services and the oxygen dose should be reduced if the saturation exceeds $92 \%$. [Grade D]

6. Because oxygenation is reduced in the supine position, fully conscious hypoxaemic patients should ideally be allowed to maintain the most upright posture possible (or the most comfortable posture for the patient) unless there are good reasons to immobilise the patient (eg, skeletal or spinal trauma). [Grade C]

\section{Clinical and laboratory assessment of hypoxaemia and hypercapnia (section 7.1)}

7. Fully trained clinicians should assess all acutely ill patients by measuring pulse, blood pressure, respiratory rate and assessing circulating blood volume and anaemia. Expert assistance from specialists in intensive care or from other disciplines should be sought at an early stage if patients are thought to have major life-threatening illnesses and clinicians should be prepared to call for assistance when necessary, including a call for a 999 ambulance in prehospital care or a call for the resuscitation team or ICU outreach team in hospital care. [Grade C-D]

8. Initial clinical assessment and subsequent monitoring of acutely unwell patients should include the use of a recognised physiological "track and trigger" system, such as the Modified Early Warning Scoring System (mEWS), and a change in this score should require medical review even if there is no change in oxygen saturation. [Grade C]

9. Oxygen saturation, "the fifth vital sign", should be checked by trained staff using pulse oximetry in all breathless and acutely ill patients (supplemented by blood gases when necessary) and the inspired oxygen concentration should be recorded on the observation chart with the oximetry result. [Grade D]

10. The presence of a normal oxygen saturation (arterial oxygen saturation measured by pulse oximetry $\left(\mathrm{SpO}_{2}\right)$ does not always negate the need for blood gas measurements because pulse oximetry will be normal in a patient with normal oxygen tension but abnormal blood $\mathrm{pH}$ or carbon dioxide tension $\left(\mathrm{PCO}_{2}\right)$ or with a low blood oxygen content due to anaemia). Blood gas measurements and full blood counts are therefore required as early as possible in all situations where these measurements may affect patient outcomes. [Grade D]

\section{Arterial and arteriolised blood gases (sections 7.1.3 and 8.4)}

11. For critically ill patients or those with shock or hypotension (systolic blood pressure $<90 \mathrm{~mm} \mathrm{Hg}$ ), the initial blood gas measurement should be obtained from an arterial specimen. However, for most patients who require blood gas sampling, either arterial blood gases or arteriolised earlobe blood gases may be used to obtain an accurate measure of
$\mathrm{pH}$ and $\mathrm{PCO}_{2}$. However, the arterial oxygen tension $\left(\mathrm{PaO}_{2}\right)$ is less accurate in earlobe blood gas samples (it underestimates the oxygen tension by $0.5-1 \mathrm{kPa}$ ), so oximetry should be monitored carefully if earlobe blood gas specimens are used. [Grade B]

12. Local anaesthesia should be used for all arterial blood gas specimens except in emergencies or if the patient is unconscious or anaesthetised. [Grade B]

13. Blood gases should be checked in the following situations: - All critically ill patients.

- Unexpected or inappropriate hypoxaemia $\left(\mathrm{SpO}_{2}<94 \%\right)$ or any patient requiring oxygen to achieve this target range. (Allowance should be made for transient dips in saturation to $90 \%$ or less in normal subjects during sleep). [Grade D]

- Deteriorating oxygen saturation or increasing breathlessness in a patient with previously stable hypoxaemia (eg, severe COPD). [Grade D]

- Any previously stable patient who deteriorates and requires a significantly increased fraction of inspired oxygen $\left(\mathrm{FIO}_{2}\right)$ to maintain a constant oxygen saturation. [Grade D]

- Any patient with risk factors for hypercapnic respiratory failure who develops acute breathlessness, deteriorating oxygen saturation or drowsiness or other symptoms of $\mathrm{CO}_{2}$ retention. [Grade D]

- Breathless patients who are thought to be at risk of metabolic conditions such as diabetic ketoacidosis or metabolic acidosis due to renal failure. [Grade D]

- Acutely breathless or critically ill patients with poor peripheral circulation in whom a reliable oximetry signal cannot be obtained. [Grade D]

- Any other evidence from the patient's medical condition that would indicate that blood gas results would be useful in the patient's management (eg, an unexpected change in "track and trigger" systems such as a sudden rise of several units in the mEWS or an unexpected fall in oxygen saturation of $3 \%$ or more, even if within the target range). [Grade D]

Oxygen use in specific illnesses

See tables 1-4 and figs 1 and 2 (and section 8 in main text)

- Critical illness requiring high levels of supplemental oxygen: see table 1 and section 8

- Serious illness requiring moderate levels of supplemental oxygen if a patient is hypoxaemic: see table 2 and section 8

- COPD and other conditions requiring controlled or low-dose oxygen therapy: see table 3 and section 8 .

- Conditions for which patients should be monitored closely but oxygen therapy is not required unless the patient is hypoxaemic: see table 4 and section 8 .

\section{Oxygen therapy in pregnancy (section 8.13.3)}

14. Women who suffer from major trauma, sepsis or acute illness during pregnancy should receive the same oxygen therapy as any other seriously ill patients, with a target oxygen saturation of $94-98 \%$. The same target range should be applied to women with hypoxaemia due to acute complications of pregnancy (eg, collapse related to amniotic fluid embolus, eclampsia or antepartum or postpartum haemorrhage). [Grade D] 
15. Women with underlying hypoxaemic conditions (eg, heart failure) should be given supplemental oxygen during labour to achieve an oxygen saturation of $94-98 \%$. [Grade D]

16. All women with evidence of hypoxaemia who are more than 20 weeks pregnant should be managed with left lateral tilt to improve cardiac output. [Grade B]

17. The use of oxygen during labour is widespread but there is evidence that this may be harmful to the fetus. The use of oxygen during labour is therefore not currently recommended in situations where the mother is not hypoxaemic (except as part of a controlled trial). [Grade A]

\section{Emergency use of oxygen in prehospital and hospital care (sections 8 and 9)}

18. Pulse oximetry must be available in all locations where emergency oxygen is being used (see also the limitations of using pulse oximetry, section 7.1.2). [Grade D]

19. Emergency oxygen should be available in primary care medical centres, preferably using oxygen cylinders with integral high-flow regulators. Alternatively, oxygen cylinders fitted with high-flow regulators (delivering over $6 \mathrm{l}$ / min) must be used. [Grade D]

20. All documents which record oximetry measurements should state whether the patient is breathing air or a specified dose of supplemental oxygen. [Grade C]

21. The oxygen saturation should be monitored continuously until the patient is stable or arrives at hospital for a full assessment. The oxygen concentration should be adjusted upwards or downwards to maintain the target saturation range. [Grade D]

22. In most emergency situations, oxygen is given to patients immediately without a formal prescription or drug order. The lack of a prescription should never preclude oxygen being given when needed in an emergency situation. However, a subsequent written record must be made of what oxygen therapy has been given to every patient (in a similar manner to the recording of all other emergency treatment). [Grade D]

23. Patients with COPD (and other at-risk conditions) who have had an episode of hypercapnic respiratory failure should be issued with an oxygen alert card and with a $24 \%$ or $28 \%$ Venturi mask. They should be instructed to show the card to the ambulance crew and emergency department staff in the event of an exacerbation. [Grade C]

24. The content of the alert card should be specified by the physician in charge of the patient's care, based on previous blood gas results. [Grade D]

25. The primary care team and ambulance service should also be informed by the responsible clinician that the patient has had an episode of hypercapnic respiratory failure and carries an oxygen alert card. The home address and ideal oxygen dose or target saturation ranges of these patients can be flagged in the ambulance control systems and disseminated to ambulance crews when required. [Grade D]

26. Out-of-hours services providing emergency primary care services should be informed by a responsible clinician that the patient has had an episode of hypercapnic respiratory failure and carries an oxygen alert card. Use of oxygen in these patients will be guided by the instructions on the alert card. [Grade D]

27. During ambulance journeys oxygen-driven nebulisers should be used for patients with asthma and may be used for patients with COPD in the absence of an air-driven compressor system. If oxygen is used for patients with known COPD, its use should be limited to $6 \mathrm{~min}$. This will deliver most of the nebulised drug dose but limit the risk of hypercapnic respiratory failure (section 10.8.2). [Grade D]

28. If a patient is suspected to have hypercapnia or respiratory acidosis due to excessive oxygen therapy, the oxygen therapy should not be discontinued but should be stepped down to $28 \%$ or $24 \%$ oxygen from a Venturi mask depending on oxygen saturation and subsequent blood gas results. [Grade C]

\section{Equipment used to deliver emergency oxygen therapy (see section 10)}

29. (a) It is recommended that the following delivery devices should be available in prehospital settings where oxygen is administered: [Grade D]

- high concentration reservoir mask (non-rebreathe mask) for high-dose oxygen therapy;

- nasal cannulae (preferably) or a simple face mask for medium-dose oxygen therapy;

- $28 \%$ Venturi mask for patients with definite or likely COPD (patients who have an oxygen alert card may have their own $24 \%$ or $28 \%$ Venturi mask);

- tracheostomy masks for patients with tracheostomy or previous laryngectomy.

(b) Most hospital patients can be managed with the same delivery device as in 29a, but $24 \%$ Venturi masks should also be available. [Grade D]

30. For many patients Venturi masks can be substituted with nasal cannulae at low flow rates $(1-21 / \mathrm{min})$ to achieve the same target range once patients have stabilised. [Grade D]

31. The flow rate from simple face masks should be adjusted between 5 and $10 \mathrm{l} / \mathrm{min}$ to achieve the desired target saturation. Flow rates below $5 \mathrm{l} / \mathrm{min}$ may cause carbon dioxide rebreathing and increased resistance to inspiration. [Grade C]

32. Patients with COPD with a respiratory rate of $>30$ breaths/min should have the flow rate set to $50 \%$ above the minimum flow rate specified for the Venturi mask and/or packaging (increasing the oxygen flow rate into a Venturi mask increases the total gas flow from the mask but does not increase the concentration of oxygen which is delivered). [Grade C]

33. Trusts should take measures to eliminate the risk of oxygen tubing being connected to the incorrect wall oxygen outlet or to outlets that deliver compressed air or other gases instead of oxygen. Air flow meters should be removed from the wall sockets or covered with a designated air outlet cover when not in use. Special care should be taken if twin oxygen outlets are in use. [Grade D]

34. Humidification is not required for the delivery of low-flow oxygen or for the short-term use of high-flow oxygen. It is not therefore required in prehospital care. Pending the results of clinical trials, it is reasonable to use humidified oxygen for patients who require high-flow oxygen systems for more than $24 \mathrm{~h}$ or who report upper airway discomfort due to dryness. [Grade B]

35. In the emergency situation humidified oxygen use can be confined to patients with tracheostomy or an artificial airway, although these patients can be managed without humidification for short periods of time (eg, ambulance journeys). [Grade D] 
36. Humidification may also be of benefit to patients with viscous secretions causing difficulty with expectoration. This benefit can be achieved using nebulised normal saline. [Grade C]

37. Bubble bottles should not be used because there is no evidence of clinically significant benefit but there is a risk of infection. [Grade C]

38. When oxygen is required by patients with prior tracheostomy or laryngectomy, a tracheostomy mask (varying the flow as necessary) should achieve the desired oxygen saturation (tables 1-4). An alternative delivery device, usually a two-piece device fitted directly to the tracheostomy tube, may be necessary if the patient deteriorates. [Grade D]

\section{Oxygen therapy during nebulised treatments (see section 10)}

39. For patients with asthma, nebulisers should be driven by piped oxygen or from an oxygen cylinder fitted with a highflow regulator capable of delivering a flow rate of $>6 \mathrm{l} / \mathrm{min}$. The patient should be changed back to his/her usual mask when nebuliser therapy is complete. If the cylinder does not produce this flow rate, an air-driven nebuliser (with electrical compressor) should be used with supplemental oxygen by nasal cannulae at $2-61 / \mathrm{min}$ to maintain an appropriate oxygen saturation level. [Grade D]

40. When nebulised bronchodilators are given to patients with hypercapnic acidosis, they should be driven by compressed air and, if necessary, supplementary oxygen should be given concurrently by nasal cannulae at $2-4 \mathrm{l} / \mathrm{min}$ to maintain an oxygen saturation of $88-92 \%$. The same precautions should be applied to patients who are at risk of hypercapnic respiratory failure prior to the availability of blood gas results. Once the nebulised treatment is completed for patients at risk of hypercapnia, controlled oxygen therapy with a fixed concentration (Venturi) device should be reinstituted. [Grade D]

- During ambulance journeys, oxygen-driven nebulisers should be used for patients with asthma and may be used for patients with COPD in the absence of an air-driven compressor system. If oxygen is used for patients with known COPD, its use should be limited to $6 \mathrm{~min}$. This will deliver most of the nebulised drug dose but limit the risk of hypercapnic respiratory failure (see recommendation 27).

\section{Prescription, administration, monitoring and discontinuation of} oxygen therapy (see sections 11 and 12)

Oxygen should always be prescribed or ordered on a designated document. In emergencies, oxygen should be given first and documented later. See recommendations 41-76 in section 11 of the main guideline for prescription, administration and monitoring of oxygen therapy and recommendations 77-84 in section 12 for guidance on meaning and discontinuation of oxygen therapy.

All primary care trusts, ambulance trusts and hospital trusts should take specific measures to institute safe and effective administration and documentation of oxygen as described in recommendations $41-84$ in sections 11 and 12 of this guideline.

Table 1 Critical illnesses requiring high levels of supplemental oxygen (see section 8.10)

- The initial oxygen therapy is a reservoir mask at $15 \mathrm{l} / \mathrm{min}$.

- Once stable, reduce the oxygen dose and aim for target saturation range of $94-98 \%$

- If oximetry is unavailable, continue to use a reservoir mask until definitive treatment is available.

- Patients with COPD and other risk factors for hypercapnia who develop critical illness should have the same initial target saturations as other critically ill patients pending the results of blood gas measurements, after which these patients may need controlled oxygen therapy or supported ventilation if there is severe hypoxaemia and/or hypercapnia with respiratory acidosis.

\section{Additional comments}

Cardiac arrest or resuscitation

Shock, sepsis, major trauma, near-drowning, anaphylaxis, major pulmonary haemorrhage Major head injury Carbon monoxide poisoning
Use bag-valve mask during active resuscitation

Aim for maximum possible oxygen saturation until the patient is stable

Also give specific treatment for the underlying condition

Grade of recommendation Grade D

Grade D

Early intubation and ventilation if comatose

Grade D

Give as much oxygen as possible using a bag-valve mask or reservoir mask. Check carboxyhaemoglobin levels

A normal or high oximetry reading should be disregarded because saturation monitors cannot differentiate between carboxyhaemoglobin and oxyhaemoglobin owing to their similar absorbances. The blood gas $\mathrm{PaO}_{2}$ will also be normal in these cases (despite the presence of tissue hypoxia) 
Table 2 Serious illnesses requiring moderate levels of supplemental oxygen if the patient is hypoxaemic (section 8.11)

- The initial oxygen therapy is nasal cannulae at 2-6 $\mathrm{l} / \mathrm{min}$ (preferably) or simple face mask at 5-10 $\mathrm{l} / \mathrm{min}$ unless stated otherwise.

- For patients not at risk of hypercapnic respiratory failure who have saturation $<85 \%$, treatment should be commenced with a reservoir mask at $10-15 \mathrm{l} / \mathrm{min}$.

- The recommended initial oxygen saturation target range is $94-98 \%$.

- If oximetry is not available, give oxygen as above until oximetry or blood gas results are available.

- Change to reservoir mask if the desired saturation range cannot be maintained with nasal cannulae or simple face mask (and ensure that the patient is assessed by senior medical staff).

- If these patients have co-existing COPD or other risk factors for hypercapnic respiratory failure, aim at a saturation of $88-92 \%$ pending blood gas results but adjust to $94-98 \%$ if the $\mathrm{PaCO}_{2}$ is normal (unless there is a history of previous hypercapnic respiratory failure requiring NIV or IPPV) and recheck blood gases after 30-60 min.

Acute hypoxaemia (cause not yet diagnosed)

Acute asthma

Pneumonia

Lung cancer

Postoperative breathlessness

Acute heart failure

Pulmonary embolism

Pleural effusions

Pneumothorax

Deterioration of lung fibrosis or other interstitial lung disease

Severe anaemia

Sickle cell crisis

\section{Additional comments}

Reservoir mask at $10-15 \mathrm{l} / \mathrm{min}$ if initial $\mathrm{SpO}_{2}<85 \%$, otherwise nasal cannulae or simple face mask

Patients requiring reservoir mask therapy need urgent clinical assessment by senior staff

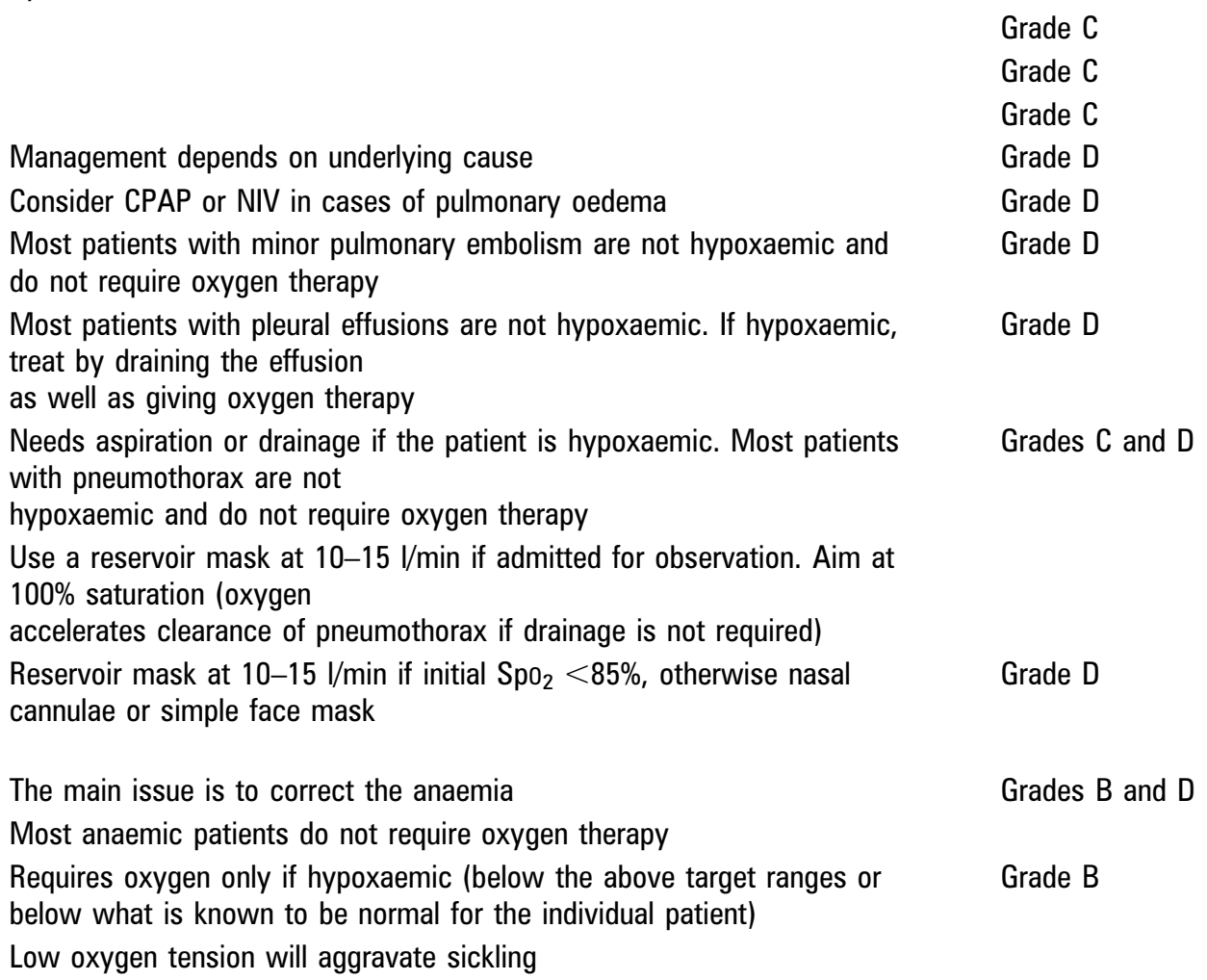

COPD, chronic obstructive pulmonary disease; CPAP, continuous positive airway pressure; IPPV, intermittent positive pressure ventilation; NIV, non-invasive ventilation; $\mathrm{PaCO}_{2}$, arterial carbon dioxide tension; $\mathrm{SpO}_{2}$, arterial oxygen saturation measured by pulse oximetry.

\section{Grade of recommendation} Grade D

Grade C
Grade C
Grade C
Grade D
Grade D
Grade D

Most patients with pleural effusions are not hypoxaemic. If hypoxaemic as well as giving oxygen therapy

Needs aspiration or drainage if the patient is hypoxaemic. Most patients with pneumothorax are not

hypoxaemic and do not require oxygen therapy

$100 \%$ saturation (oxygen

accelerates clearance of pneumothorax if drainage is not required)

cannulae or simple face mask 
Table 3 COPD and other conditions requiring controlled or low-dose oxygen therapy (section 8.12)

- Prior to availability of blood gases, use a $28 \%$ Venturi mask at $4 \mathrm{l} / \mathrm{min}$ and aim for an oxygen saturation of $88-92 \%$ for patients with risk factors for hypercapnia but no prior history of respiratory acidosis. [Grade D]

- Adjust target range to $94-98 \%$ if the $\mathrm{Paco}_{2}$ is normal (unless there is a history of previous NIV or IPPV) and recheck blood gases after 30 60 min [Grade D]

- Aim at a prespecified saturation range (from alert card) in patients with a history of previous respiratory acidosis. These patients may have their own Venturi mask. In the absence of an oxygen alert card but with a history of previous respiratory failure (use of NIV or IPPV), treatment should be commenced using a $28 \%$ oxygen mask at $4 \mathrm{l} / \mathrm{min}$ in prehospital care or a $24 \%$ Venturi mask at $2-4 \mathrm{l} / \mathrm{min}$ in hospital settings with an initial target saturation of $88-92 \%$ pending urgent blood gas results. [Grade D]

- If the saturation remains below $88 \%$ in prehospital care despite a $28 \%$ Venturi mask, change to nasal cannulae at 2-6 $1 /$ min or a simple mask at $5 \mathrm{l} / \mathrm{min}$ with target saturation of $88-92 \%$. All at-risk patients with alert cards, previous NIV or IPPV or with saturation $<88 \%$ in the ambulance should be treated as a high priority. Alert the A\&E department that the patient requires immediate senior assessment on arrival at the hospital. [Grade D]

- If the diagnosis is unknown, patients aged $>50$ years who are long-term smokers with a history of chronic breathlessness on minor exertion such as walking on level ground and no other known cause of breathlessness should be treated as if having COPD for the purposes of this guideline. Patients with COPD may also use terms such as chronic bronchitis and emphysema to describe their condition but may sometimes mistakenly use "asthma". FEV 1 should be measured on arrival in hospital if possible and should be measured at least once before discharge from hospital in all cases of suspected COPD. [Grade D]

- Patients with a significant likelihood of severe COPD or other illness that may cause hypercapnic respiratory failure should be triaged as very urgent and blood gases should be measured on arrival in hospital. [Grade D]

- Blood gases should be rechecked after 30-60 min (or if there is clinical deterioration) even if the initial $\mathrm{PacO}_{2}$ measurement was normal. [Grade D]

- If the $\mathrm{PaCO}_{2}$ is raised but $\mathrm{pH}$ is $\geqslant 7.35\left(\left[\mathrm{H}^{+}\right] \leqslant 45 \mathrm{nmol} / \mathrm{l}\right)$, the patient has probably got long-standing hypercapnia; maintain target range of 88-92\% for these patients. Blood gases should be repeated at 30-60 min to check for rising $\mathrm{PaCO}_{2}$ or falling pH. [Grade D]

- If the patient is hypercapnic $\left(\mathrm{PacO}_{2}>6 \mathrm{kPa}\right.$ or $\left.45 \mathrm{~mm} \mathrm{Hg}\right)$ and acidotic $\left(\mathrm{pH}<7.35\right.$ or $\left.\left[\mathrm{H}^{+}\right]>45 \mathrm{nmol} / \mathrm{l}\right)$ consider non-invasive ventilation, especially if acidosis has persisted for more than 30 min despite appropriate therapy. [Grade A]

\section{COPD}

Exacerbation of $\mathrm{CF}$

Chronic neuromuscular disorders

Chest wall disorders

Morbid obesity

\section{Additional comments}

May need lower range if acidotic or if known to be very sensitive to oxygen therapy. Ideally use alert cards to guide treatment based on previous blood gas results. Increase flow by $50 \%$ if respiratory rate is $>30$ (see recommendation 32)

Admit to regional CF centre if possible; if not, discuss with regional centre or manage according to protocol agreed with regional CF centre

Ideally use alert cards to guide therapy. Increase flow by $50 \%$ if respiratory rate is $>30$ (see recommendation 32 )

May require ventilatory support. Risk of hypercapnic respiratory failure

For acute neuromuscular disorders and subacute conditions such as Guillain-Barré syndrome (see table 4)
Grade D

Grade of recommendation Grade C

Grade D

Grade D

Grade D

$\mathrm{CF}$, cystic fibrosis; $\mathrm{COPD}$, chronic obstructive pulmonary disease; CPAP, continuous positive airway pressure; IPPV, intermittent positive pressure ventilation; NIV, non-invasive ventilation; $\mathrm{PaCO}_{2}$, arterial carbon dioxide tension; $\mathrm{SpO}_{2}$, arterial oxygen saturation measured by pulse oximetry. 
Table 4 Conditions for which patients should be monitored closely but oxygen therapy is not required unless the patient is hypoxaemic (section 8.13)

- If hypoxaemic, the initial oxygen therapy is nasal cannulae at $2-6 \mathrm{l} / \mathrm{min}$ or simple face mask at $5-10 \mathrm{l} / \mathrm{min}$ unless saturation is $<85 \%$ (use reservoir mask) or if at risk from hypercapnia (see below).

- The recommended initial target saturation range, unless stated otherwise, is $94-98 \%$

- If oximetry is not available, give oxygen as above until oximetry or blood gas results are available

- If patients have COPD or other risk factors for hypercapnic respiratory failure, aim at a saturation of $88-92 \%$ pending blood gas results but adjust to $94-98 \%$ if the $\mathrm{PacO}_{2}$ is normal (unless there is a history of respiratory failure requiring NIV or IPPV) and recheck blood gases after $30-60 \mathrm{~min}$

\section{Additional comments}

Myocardial infarction and acute coronary syndromes

Stroke

Pregnancy and obstetric emergencies

Hyperventilation or dysfunctional breathing

Most poisonings and drug overdoses (see table 1 for carbon monoxide poisoning)

Poisoning with paraquat or bleomycin

Metabolic and renal disorders

Acute and subacute neurological and muscular conditions producing muscle weakness recommended in this condition patients)
Most patients with acute coronary artery syndromes are not hypoxaemic and the benefits/harms of oxygen therapy are unknown in such cases

Most stroke patients are not hypoxaemic. 0xygen therapy may be harmful for non-hypoxaemic patients with mild to moderate strokes.

Oxygen therapy may be harmful to the fetus if the mother is not hypoxaemic (see recommendations 14-17)

Exclude organic illness. Patients with pure hyperventilation due to anxiety or panic attacks are unlikely to require oxygen therapy

Rebreathing from a paper bag may cause hypoxaemia and is not

Hypoxaemia is more likely with respiratory depressant drugs, give antidote if available (eg, naloxone for opiate poisoning)

Check blood gases to exclude hypercapnia if a respiratory depressant drug has been taken. Avoid high blood oxygen levels in cases of acid aspiration as there is theoretical evidence that oxygen may be harmful

Monitor all potentially serious cases of poisoning in a level 2 or level 3 environment (high dependency unit or ICU)

Patients with paraquat poisoning or bleomycin lung injury may be harmed by supplemental oxygen

Avoid oxygen unless the patient is hypoxaemic

Target saturation is $88-92 \%$

Most do not need oxygen (tachypnoea may be due to acidosis in these

These patients may require ventilatory support and they need careful monitoring which includes spirometry. If the patient's oxygen level falls below the target saturation, they need urgent blood gas measurements and are likely to need ventilatory support
Grade of recommendation

Grade D

Grade B

Grades A-D

Grade C

Grade D

Grade C

Grade D

Grade C

COPD, chronic obstructive pulmonary disease; ICU, intensive care unit; IPPV, intermittent positive pressure ventilation; NIV, non-invasive ventilation; $\mathrm{PaCO}_{2}$, arterial carbon dioxide tension; $\mathrm{SpO}_{2}$, arterial oxygen saturation measured by pulse oximetry. 


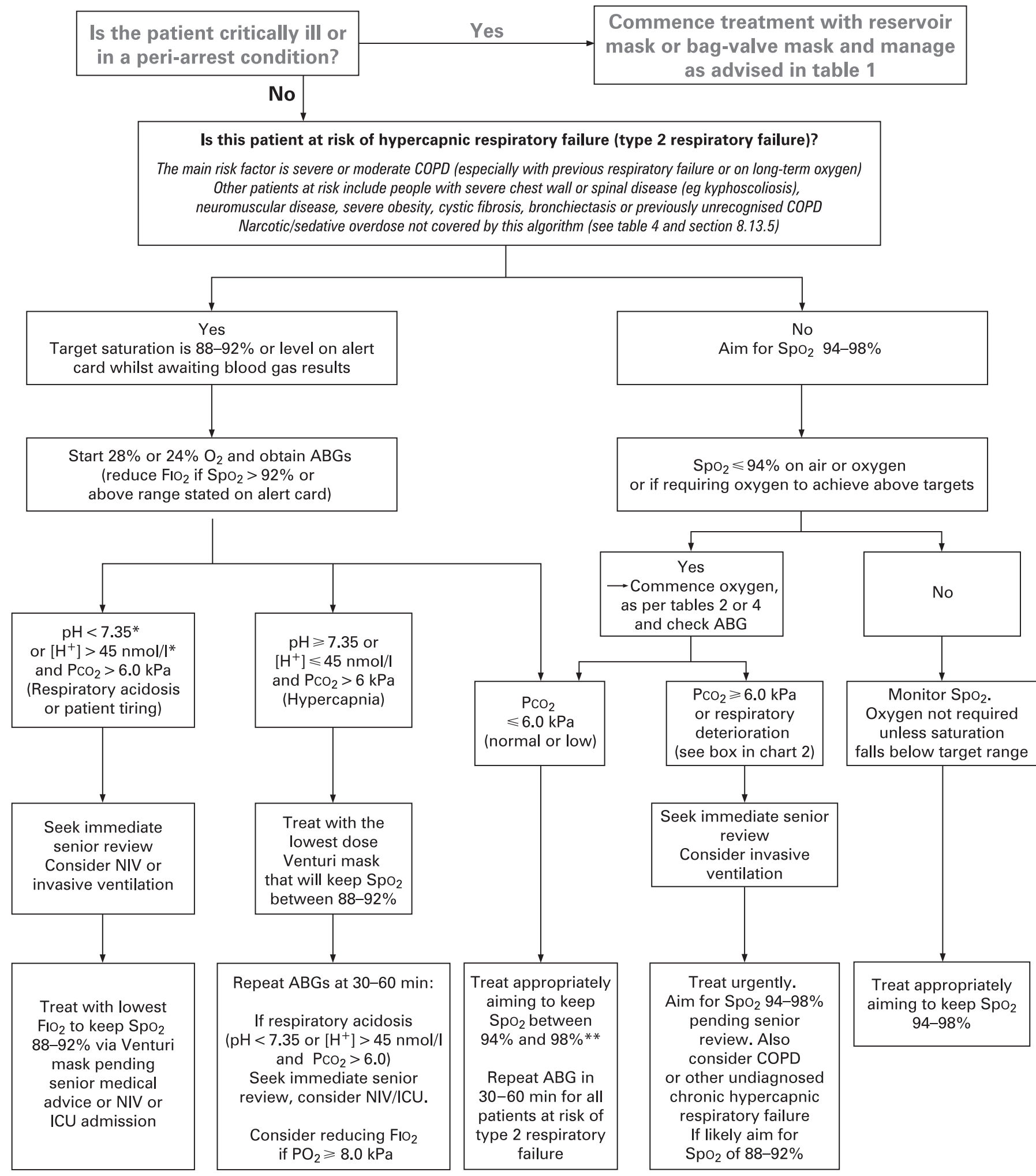

Any increase in $\mathrm{FIO}_{2}$ must be followed by repeat $\mathrm{ABGs}$ in $1 \mathrm{~h}$ (or sooner if conscious level deteriorates)

*If $\mathrm{pH}$ is $<7.35\left(\left[\mathrm{H}^{+}\right]>45 \mathrm{nmol} / /\right)$ with normal or low $\mathrm{PacO}_{2}$, investigate and treat for metabolic acidosis and keep Spo $294-98 \%$

**Patients previously requiring NIV or IPPV should have a target range of $88-92 \%$, even if the initial $\mathrm{PaCO}_{2}$ is normal.

Figure 1 Chart 1: Oxygen prescription for acutely hypoxaemic patients in hospital. ABG, arterial blood gas; COPD, chronic obstructive pulmonary disease; $\mathrm{FIO}_{2}$, fraction of inspired oxygen; ICU, intensive care unit; NIV, non-invasive ventilation; $\mathrm{PCO}_{2}$, carbon dioxide tension; $\mathrm{SpO}_{2}$, arterial oxygen saturation measured by pulse oximetry. 
See patient's drug chart and chart 1 and tables 1-4 for starting dose and target saturation

Choose the most suitable delivery system and flow rate

Titrate oxygen up or down to maintain the target oxygen saturation.

The table below shows available options for stepping dosage up or down. The chart does not imply any equivalence of dose between Venturi masks and nasal cannulae.

Allow at least 5 minutes at each dose before adjusting further upwards or downwards (except with major and sudden fall in saturation).

Once your patient has adequate and stable saturation on minimal oxygen dose, consider discontinuation of oxygen therapy.

Venturi $24 \% 2-41 / \mathrm{min}^{*}$

Seek medical advice
if patient appears
to need increasing
oxygen therapy or if
there is a rising
mEWS or Track and
Trigger score

\section{All patients must have} $A B G$ or earlobe blood gases (ELBG) within $1 \mathrm{~h}$ of requiring increased oxygen dose

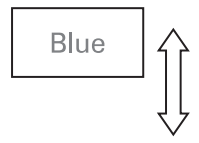

Venturi $28 \% 4-61 / \mathrm{min}$

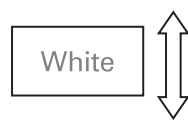

Venturi 35\% 8-10 I/min

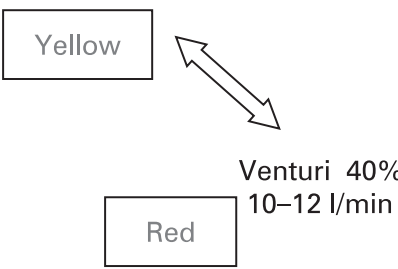

Red

Venturi $60 \%$ $12-15 \mathrm{l} / \mathrm{min}$
Nasal cannulae $1 \mathrm{l} / \mathrm{min}$

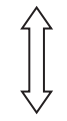

Nasal cannulae $2 \mathrm{l} / \mathrm{min}$

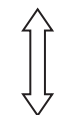

Nasal cannulae $4 \mathrm{l} / \mathrm{min}$

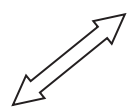

or simple face mask at $5-6 \mathrm{l} / \mathrm{min}$<smiles>c1ccccc1</smiles>

or simple face mask

$7-10 \mathrm{l} / \mathrm{min}$<smiles>[CH]C[Tl]</smiles>

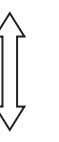

Reservoir mask at $15 \mathrm{l} / \mathrm{min}$ oxygen flow<smiles>[C]=C</smiles>

If reservoir mask required, seek senior medical input immediately

* For Venturi masks, the higher flow rate is required if the respiratory rate is $>30$

Patients in a peri-arrest situation and critically ill patients should be given maximal oxygen therapy via reservoir mask or bag-valve mask whilst immediate medical help is arriving

(except for patients with COPD with known oxygen sensitivity recorded in patient's case notes and drug chart or in the EPR: keep saturation at $88-92 \%$ for this subgroup of patients)

Figure 2 Chart 2: Flow chart for oxygen administration on general wards in hospitals. ABG, arterial blood gas; EPR, electronic patient record; EWS, Early Warning Score; $\mathrm{SpO}_{2}$, arterial oxygen saturation measured by pulse oximetry. 


\section{HIERARCHY OF EVIDENCE AND GRADING OF RECOMMENDATIONS}

\section{Levels of evidence and grades of recommendation}

Levels of evidence and grades of recommendation are based on the levels of evidence used in the NICE COPD guideline ${ }^{25}$ (see tables 5 and 6 ). For most of the topics covered by the guideline there were either no randomised trials or just a handful of observational studies. Members of the group reviewed the evidence for each topic and assigned the most appropriate grading which was usually grade $\mathrm{C}$ evidence (case-control or cohort studies) or grade $\mathrm{D}$ evidence (expert opinion or case reports).

Each recommendation has been allocated a grading which directly reflects the hierarchy of evidence upon which it is based.

Please note that the hierarchy of evidence and the recommendation gradings relate to the strength of the literature, not to clinical importance. This is especially important in the field of oxygen therapy where there are very few controlled trials.

Table 5 Hierarchy of evidence

\begin{tabular}{ll}
\hline $\begin{array}{l}\text { Level of } \\
\text { evidence }\end{array}$ & Type of evidence \\
\hline la & $\begin{array}{l}\text { Evidence from systematic reviews or meta-analysis } \\
\text { of randomised controlled trials } \\
\text { Evidence from at least one randomised controlled } \\
\text { trial }\end{array}$ \\
Ib & $\begin{array}{l}\text { Evidence from at least one controlled study without } \\
\text { randomisation }\end{array}$ \\
Ila & $\begin{array}{l}\text { Evidence from at least one other type of quasi- } \\
\text { experimental study } \\
\text { IIb }\end{array}$ \\
III & $\begin{array}{l}\text { Evidence from non-experimental descriptive studies } \\
\text { such as comparative studies, correlation studies and } \\
\text { case-control studies } \\
\text { Evidence from expert committee reports or opinions } \\
\text { and/or clinical experience of respected authorities }\end{array}$ \\
\hline
\end{tabular}

Table 6 Grading of recommendations

\begin{tabular}{ll}
\hline Level of evidence & Type of evidence \\
\hline A & Based on hierarchy I evidence \\
B & $\begin{array}{l}\text { Based on hierarchy II evidence or extrapolated from hierarchy I } \\
\text { evidence }\end{array}$ \\
C & $\begin{array}{l}\text { Based on hierarchy III evidence or extrapolated from hierarchy I or } \\
\text { II evidence }\end{array}$ \\
D & $\begin{array}{l}\text { Directly based on hierarchy IV evidence or extrapolated from } \\
\text { hierarchy I, II or III evidence }\end{array}$
\end{tabular}

\section{SECTION 1: INTRODUCTION}

\subsection{Clinical context}

Oxygen is probably the commonest drug to be used in the care of patients who present with medical emergencies. Currently, ambulance teams and emergency department teams are likely to give oxygen to virtually all breathless patients and to a large number of patients with other conditions such as ischaemic heart disease, sepsis or trauma. The North West Ambulance Service serves a population of about 7.25 million people and transports about 700000 people to hospital emergency departments each year. About 34\% of these journeys involve oxygen use at some stage. ${ }^{1}$ This translates to about two million instances of emergency oxygen use per annum by all UK ambulance services, with further use in patients' homes, GP surgeries and in hospitals.

At present, oxygen is administered for three main indications of which only one is evidence-based. First, oxygen is given to correct hypoxaemia as there is good evidence that severe hypoxaemia is harmful. Second, oxygen is administered to ill patients in case they might become hypoxaemic. Recent evidence suggests that this practice may actually place patients at increased risk if severe hypoxaemia does actually develop (see section 6.3.4). Third, a very high proportion of medical oxygen is administered because most clinicians believe that oxygen can alleviate breathlessness. However, there is no evidence that oxygen relieves breathlessness in non-hypoxaemic patients and there is evidence of lack of effectiveness in non-hypoxaemic breathless patients with chronic obstructive pulmonary disease (COPD) and advanced cancer (see sections 6.6 and 8.11.4).

\subsection{Prescription of oxygen}

Most clinicians who deal with medical emergencies will encounter adverse incidents and occasional deaths due to underuse and overuse of oxygen. Audits of oxygen use and oxygen prescription have shown consistently poor performance in many countries. ${ }^{2-8}$ One major problem is that healthcare professionals receive conflicting advice about oxygen use from different "experts" during their training and during their clinical careers, and many are confused about the entire area of oxygen prescription and use.

\subsection{Need for a guideline for emergency oxygen therapy and purpose of the guideline}

There is considerable controversy concerning the benefits and risks of oxygen treatment in virtually all situations where oxygen is used. Unfortunately, this is an area of medicine where there are many strongly-held beliefs but very few randomised controlled trials. The only published UK guideline for emergency oxygen therapy is the North West Oxygen Guideline published in 2001, based on a systematic literature review by the same authors. ${ }^{9}{ }^{10}$ Against this background, the Standards of Care Committee of the British Thoracic Society (BTS) established a working party in association with 21 other societies and colleges listed at the front of this document. The objective was to produce an evidence-based and up-to-date guideline for emergency oxygen use in the UK.

\subsection{Intended users of the guideline and scope of the guideline}

This guideline is intended for use by all healthcare professionals who may be involved in emergency oxygen use. This will include ambulance staff, paramedics, doctors, nurses, midwives, physiotherapists, pharmacists and all other healthcare professionals who may deal with ill or breathless patients. 
Specific versions of this guideline will be available on the BTS website for use in the following situations:

- Hospital use

- Primary care use

- Ambulance use

- Version for use by nursing staff

These abbreviated versions of the guideline will contain the key recommendations and tables and charts that are relevant to the particular situation. The "mini-guidelines" can be downloaded by health care trusts for use on trust intranets and to produce paper versions of the guideline for key staff.

\subsection{Areas covered by this guideline}

The guideline will address the use of oxygen in three main categories of adult patients in the prehospital and hospital setting:

- Critically ill or hypoxic patients.

- Patients at risk of hypoxaemia.

- Non-hypoxic patients who might benefit from oxygen (eg, carbon monoxide poisoning).

\subsection{Areas not covered by this guideline}

- Oxygen use in paediatrics: the present guideline applies only to subjects aged $\geqslant 16$ years.

- Oxygen use for high altitude activities.

- Oxygen use during air travel.

- Underwater diving and diving accidents.

- Oxygen use in animal experiments.

- Oxygen use during surgery and anaesthesia or during endoscopy.

- Oxygen use in high-dependency units.

- Oxygen use in intensive care units.

- Interhospital level 3 transfers.

- Hyperbaric oxygen.

- Use of heliox mixtures.

- Use of nitrous oxide/oxygen mixtures (eg, Entonox).

- Respiratory support techniques including intubation, invasive ventilation, non-invasive ventilation (NIV) and continuous positive airway pressure (CPAP).

- Self-initiated use of oxygen by patients who have home oxygen for any reason (this is covered by the guidelines for home oxygen use).

- Ongoing care of hypoxaemic patients at home.

\subsection{Limitations of the guideline}

This guideline is based on the best available evidence concerning oxygen therapy. However, a guideline can never be a substitute for clinical judgement in individual cases. There may be cases where it is appropriate for clinicians to act outwith the advice contained in this guideline because of the needs of individual patients. Furthermore, the responsibility for the care of individual patients rests with the clinician in charge of the patient's care and the advice offered in this guideline must, of necessity, be of a general nature and should not be relied upon as the only source of advice in the treatment of individual patients. In particular, this guideline gives very little advice about the management of the many medical conditions that may cause hypoxaemia (apart from the specific issue of managing the patients' hypoxaemia). Readers are referred to other guidelines for advice on the management of specific conditions such as COPD, pneumonia, heart failure, etc. Some of these disease-specific guidelines suggest slightly different approaches to emergency oxygen therapy whereas the present guideline aims to provide simple all-embracing advice. All differences involving oxygen therapy for common medical emergencies are discussed in detail in section 10 of this guideline.

\section{SECTION 2: METHODOLOGY OF GUIDELINE PRODUCTION 2.1 Establishment of guideline team}

The need for a national guideline for emergency oxygen use was recognised by the BTS Standards of Care Committee in 2003. A working party was established with representatives from a wide range of professions involved in oxygen therapy and a lay representative (see full list of guideline group members in section 16). The original group was expanded in 2006 because it became clear that the development and implementation of a national guideline would require input from a very wide range of professional groups. Most development and editing of the guideline took place subsequent to this expansion of the group. The group agreed the remit of this guideline and a series of key questions as shown below. The group devised a search strategy for relevant studies. A Medline search for "oxygen" yielded over a quarter of a million "hits", most of which were not relevant to this guideline. For this reason, the BTS commissioned the Centre for Reviews and Dissemination and Centre for Health Economics at the University of York to undertake bespoke literature searches using the search strategies shown in detail in Appendix 14 on the BTS website (www.brit-thoracic.org.uk).

\subsection{Summary of key questions}

Key question 1: Physiology and pathophysiology of oxygen

- What are the dangers of hypoxia/hypoxaemia (ie, what happens to the human body)?

- What level of hypoxaemia is dangerous to all patients (even healthy adults)?

- What level of hypoxaemia is dangerous to vulnerable groups (eg, ischaemic heart disease, stroke, elderly)?

- Repeat the above searches with additional key words: elderly, stroke, myocardial infarction, heart failure, chronic obstructive pulmonary disease (COPD), trauma, renal failure.

- Same questions for hypercarbia/hypercapnia:

- Search for "hypercapnia" combined with terms implying a harmful outcome (death/tissue injury/brain damage/ coma).

- What level of hypercapnia is dangerous to all patients?

- What level of hypercapnia is dangerous to vulnerable groups (as above)?

- Same questions for respiratory acidosis:

- Search for "respiratory acidosis" combined with terms implying a harmful outcome (death/tissue injury/brain damage/coma).

- What level of respiratory acidosis is dangerous to all patients?

- What level of respiratory acidosis is dangerous to vulnerable groups (as above)?

Key question 2: Clinical aspects of hypoxaemia and oxygen therapy for common medical emergencies

- How to assess hypoxaemia (clinical, early warning score systems, oximetry, arterial and capillary blood gases).

- How to assess hypercarbia/hypercapnia. 
- Use of oxygen to relieve symptomatic breathlessness.

- Use of oxygen in acute COPD.

- Use of oxygen in acute asthma.

- Use of oxygen in pneumonia.

- Use of oxygen for pulmonary embolus.

- Use of oxygen in trauma.

- Use of oxygen in heart failure.

- Use of oxygen in myocardial infarction.

- Use of oxygen in angina.

- Use of oxygen for other patients with less common conditions were searched individually (eg, cystic fibrosis, muscular dystrophy, motor neurone disease, severe kyphoscoliosis, anaphylaxis, hyperventilation).

Key question 3: Oxygen prescription, oxygen delivery systems and oxygen transport

- Oxygen carriage in transport (practical issues; safety issues).

- Oxygen delivery systems in ambulances.

- Prescription of oxygen.

- Local hospital guidelines for oxygen use.

- Oxygen delivery systems in hospitals.

- Advantages/disadvantages of each delivery system (Venturi masks, simple face masks, nasal cannulae, high-flow masks such as non-rebreathing reservoir masks).

- Use of oxygen-driven nebulisers.

- Use of "alert cards", alert bracelets or similar hazard warning systems for patients who are known to be at risk of hypercapnia.

\subsection{How the evidence was assimilated into the guideline}

The initial search strategy was devised at two meetings of the group in 2004 and 2005. The searches in October 2005 yielded 3306 papers, the abstracts of which were checked for relevance by group members. One hundred and eighty-four of these abstracts were considered to be relevant to the present guideline. Full reprints of all relevant papers were obtained. Further references were obtained from the group's personal literature collections and from the references contained within the papers which the search yielded and by focused literature searches by members of the guideline group. The group continued to monitor the literature up to the end of 2007 for important new publications or very high quality abstracts from international meetings that were thought to be relevant to this guideline.

The group was divided into three subgroups to work on specific areas of oxygen use: (1) emergency care; (2) hospital care; (3) oxygen physiology and devices. Evidence from the literature searches was graded according to the levels of evidence used in the NICE COPD guideline (see tables 5 and 6).

The Guideline Development Group corresponded by email on a regular basis (usually at least once weekly) for most of 2006 to discuss the evidence and to produce an initial outline of the guideline and its key recommendations. The guideline was consolidated over the course of 2006 and early 2007 with each section being led by nominated group members but taking into account feedback from the complete group. Meetings of the full group were held in February 2006, September 2006 and February 2007. Between November 2006 and February 2007 the group had an intensive review and email discussion of one guideline section per week with the objective of achieving a consensus on all of the key points before the final meeting of the group in February 2007. The draft guideline was first submitted to the BTS Standards of
Care Committee in March 2007. The guideline was further refined by email discussion following comments by this committee. The resulting draft was sent to 17 peer reviewers (see section 17) and was posted on the BTS website for 4 weeks in August 2007 and comments were invited. The document was then sent back to the Standards of Care Committee and the 21 other Societies and Colleges for endorsement.

\subsection{Piloting the guideline}

The principles of the guideline (target saturation ranges, etc) have been piloted since 2004 at Salford Royal University Hospital and Southend University Hospital. The pilot projects have included the following elements:

- Discussion with senior colleagues and management to agree the need for an oxygen guideline (and the content).

- Trust-wide introduction of the agreed hospital policy.

- Educational programme for doctors, nurses and other users of oxygen.

- Designing prescription charts and patient observation charts to facilitate the standardisation of oxygen therapy (charts 3 and 4 in figs 17 and 18 in the guideline).

- Production of a detailed implementation document which has become hospital policy in both hospitals (web appendix 3).

- The charts which are necessary to guide the prescription and administration of oxygen (charts 1 and 2 in figs 1 and 2) have been piloted successfully at both hospitals.

- The educational materials and lecture presentations in web appendix 9 have been piloted in both hospitals.

There was a lot of discussion with colleagues about the ideal target saturation range and about how to implement safe oxygen prescribing. These issues should not arise with implementation of this national guideline as the key issues are already agreed by all of the relevant specialties and are as evidence-based as is possible. Implementation proceeded smoothly at both hospitals and audit showed improved practice. However, a lot of effort is required to maintain good quality prescribing of oxygen and the role of "oxygen champions" has been piloted successfully in both hospitals (see section 14.6).

\subsection{Planned review and updating of the guideline}

The guideline will be reviewed by the BTS and by the endorsing organisations within 5 years from publication (2013).

\section{SECTION 3: NORMAL VALUES AND DEFINITIONS}

- Normal blood levels of oxygen and carbon dioxide.

- Normal oxygen saturation $\left(\mathrm{SaO}_{2}\right)$ and normal blood $\mathrm{pH}$.

- Definitions of hypoxaemia, hypoxia, hypercapnia, acidosis, respiratory failure.

Oxygen is essential for mammalian life; severe hypoxaemia such as that seen during cardiac arrest, suffocation or drowning will cause loss of consciousness, rapid organ failure and death. Oxygen is carried in the bloodstream bound to the haemoglobin molecule and delivered to the tissues. Oxygen demand and oxygen delivery increase during exercise and reduce during rest and sleep.

\subsection{Blood levels of oxygen and carbon dioxide in health and disease}

The human lung delivers oxygen to the blood and removes carbon dioxide. Several mechanisms exist to regulate breathing in such a way that both gases are maintained within quite a narrow range. 


\subsubsection{Normal ranges for oxygen saturation $\left(\mathrm{SaO}_{2}\right)$ and oxygen tension} $\left(\mathrm{PaO}_{2}\right)$ in the blood at sea level

For adults aged $<70$ years, the two standard deviation (2SD) range for $\mathrm{SaO}_{2}$ is approximately $94-98 \%$ at sea level but this may decline gradually within this age range. ${ }^{11}$ The normal range for $\mathrm{PaO}_{2}$ in the blood in seated adults at sea level is shown in table 7. However, the $\mathrm{PaO}_{2}$ is $0.8 \mathrm{kPa}(6 \mathrm{~mm} \mathrm{Hg})$ lower in the supine position than in the upright position ${ }^{12}$ and most emergency measurements are made in the supine position.

\subsubsection{Oxygen saturation in elderly patients}

The mean $\mathrm{SaO}_{2}$ may be lower in older people than in young adults. However, it is difficult to dissociate the effects of advancing age from the effects of the diseases that become commoner in old age. Some papers have reported a fall in the blood $\mathrm{PaO}_{2}$ in older subjects but others have failed to confirm this observation. ${ }^{13-15}$ The mean $\mathrm{SaO}_{2}$ in seated adults aged $>64$ years in one published study was $95.5 \%$ compared with $96.9 \%$ in adults aged 18-24 years, and the standard deviation was wider in the older age group with a 2SD range of $92.7-98.3 \%$ (table 7). ${ }^{11}$ The mean (SD) $\mathrm{SaO}_{2}$ for recumbent healthy men aged $\geqslant 70$ years in another study was 95.3 (1.4)\% giving a 2SD range of $92.5-98.1 \%$ for men of this age. ${ }^{13}$ The mean (SD) $\mathrm{SaO}_{2}$ was 94.8 (1.7)\% for recumbent healthy women aged $\geqslant 70$ years with a $2 \mathrm{SD}$ range of 91.5-98.2\%. The authors of this study did not observe any agerelated decline in $\mathrm{SaO}_{2}$ beyond the age of 70 years. The mean $\mathrm{SaO}_{2}$ in this study of approximately $95.0 \%$ for recumbent healthy men and women aged $\geqslant 70$ years was below the normal range for seated healthy young adults. The mean $\mathrm{PaO}_{2}$ in elderly subjects in this study was $10.3 \mathrm{kPa}$ for men and $9.8 \mathrm{kPa}$ for women, which is lower than two other studies which reported mean $\mathrm{PaO}_{2}$ values of $11.2 \mathrm{kPa}$ and $11.1 \mathrm{kPa}$ in healthy elderly subjects. ${ }^{14}{ }^{16}$ Some of these differences are probably due to different selection of subjects, but there are also variations in the results obtained by different blood gas analysers. ${ }^{17}$ Unfortunately there are no published data which can provide a normal range for the $\mathrm{SaO}_{2}$ in the elderly population in the UK. However, an as yet unpublished audit of 320 stable hospital patients in Salford and Southend with no history of lung disease found a mean (SD) $\mathrm{SaO}_{2}$ of $96.7(1.77) \%$ (2SD range $95.2-100 \%)$ in patients aged $\geqslant 71$ years (R O'Driscoll, A Davison, L Ward, personal communication). These values were measured by pulse oximetry in UK hospitals in 2008 and are more likely to represent the expected normal range of pulse oximetry measurements in the elderly UK population than

Table 7 Mean (SD) $\mathrm{PaO}_{2}$ and $\mathrm{SaO}_{2}$ values (with range) in $\mathrm{kPa}$ and $\mathrm{mm} \mathrm{Hg}$

\begin{tabular}{lllll}
\hline Age & $\begin{array}{l}\text { Mean (SD) } \mathrm{PaO}_{2} \\
(\mathbf{k P a} \text { and } \mathbf{m m ~ H g})\end{array}$ & $\begin{array}{l}\text { Range } \pm \mathbf{2 S D} \mathrm{PaO}_{2} \\
(\mathbf{k P a} \text { and } \mathbf{m m ~ H g})\end{array}$ & $\begin{array}{l}\mathrm{Mean}^{(S D)} \\
\mathrm{Sa0}_{2}(\%)\end{array}$ & $\begin{array}{l}\mathrm{SaO}_{2} \\
\pm 2 S D\end{array}$ \\
\hline $18-24$ & $13.4(0.71)$ & $11.98-14.82$ & $96.9(0.4)$ & $96.1-97.7$ \\
& $99.9(5.3)$ & $89.3-110.5$ & & \\
$25-34$ & $13.4(0.66)$ & $12.08-14.72$ & $96.7(0.7)$ & $95.3-98.1$ \\
& $99.8(4.9)$ & $90-109.6$ & & \\
$35-44$ & $13.18(1.02)$ & $11.14-15.22$ & $96.7(0.6)$ & $95.5-97.9$ \\
& $98.3(7.6)$ & $83.1-113.5$ & & \\
$45-54$ & $13.0(1.07)$ & $10.86-15.14$ & $96.5(1)$ & $94.4-98.5$ \\
& $97(8)$ & $81-113$ & & \\
$55-64$ & $12.09(0.60)$ & $10.89-13.29$ & $95.1(0.7)$ & $94.5-97.3$ \\
& $90.24 .5)$ & $81.2-99.2$ & & \\
$>64$ & $11.89(1.43)$ & $9.02-14.76$ & $95.5(1.4)$ & $92.7-98.3$ \\
& $88.7(10.7)$ & $67.3-110.1$ & & \\
\hline
\end{tabular}

$\mathrm{PaO}_{2}$, arterial oxygen tension; $\mathrm{SaO}_{2}$, arterial oxygen saturation.

Values shown for seated healthy men and women non-smoking volunteers at sea level (adapted from Crapo et $\mathrm{al}^{11}$ ). previous North American studies based on blood gas estimations. The variation with age, sex and posture makes it difficult to give a precise target range that will apply to all adults who might require oxygen therapy, but the guideline development committee believe that a target range of $94-98 \%$ will achieve normal or near-normal $\mathrm{SaO}_{2}$ for most adults in the UK.

- Normal daytime haemoglobin $\mathrm{SaO}_{2}$ is $96-98 \%$ in young adults in the seated position at sea level but the lower limit falls slightly with age and is about $95 \%$ in adults aged $>70$ years. [Evidence III]

\subsubsection{0xygen saturation at altitude}

The partial pressure of oxygen in the atmosphere is substantially lower at high altitude, even at altitudes where large populations live. The $\mathrm{SaO}_{2}$ at a given altitude varies with age, sex, ethnic group and degree of acclimatisation to altitude. For example, a sample of 3812 people of all ages living in Tibet at an altitude of about $4000 \mathrm{~m}$ had a mean $\mathrm{SaO}_{2}$ of only $88.2 \%$, but people native to the Andes had an $\mathrm{SaO}_{2}$ about $2.6 \%$ higher than Tibetans living at the same altitude. ${ }^{18} 19$ Millions of people live at these altitudes with $\mathrm{SaO}_{2}$ values that would cause serious concern at sea level. The city of La Paz in Bolivia has a mean altitude of $3600 \mathrm{~m}$ and a population of approximately 1.5 million people. The $\mathrm{SaO}_{2}$ of climbers on Mount Everest $(8848 \mathrm{~m})$ can fall below $70 \% .^{20}$ Sudden exposure to altitudes above about $4000 \mathrm{~m}$ can cause mountain sickness, high altitude pulmonary oedema and high altitude cerebral oedema in unacclimatised individuals. Long-term exposure to high altitude (or to hypoxaemia for any other reason) can lead to pulmonary hypertension.

\subsubsection{0xygen saturation in acute and chronic disease}

If the blood oxygen level falls to extremely low levels for even a few minutes (eg, during cardiac arrest), tissue hypoxia and cell death will occur, especially in the brain. The brain appears to be the most vulnerable organ during profound hypoxaemia; brain malfunction is the first symptom of hypoxia and brain injury is the most common long-term complication in survivors of cardiac arrests and other episodes of profound hypoxaemia. Sudden exposure to low arterial oxygen saturations below about $80 \%$ can cause altered consciousness even in healthy subjects. It is likely that other organs in patients with critical illness or chronic organ damage are vulnerable to the risk of hypoxic tissue injury at oxygen levels above $80 \%$.

Most experts emphasise the importance of keeping the $\mathrm{SaO}_{2}$ above $90 \%$ for most acutely ill patients. ${ }^{21-24}$ However, the degree of hypoxia that will cause cellular damage is not well established and probably is not an absolute value. Healthy older adults, for instance, have lower $\mathrm{SaO}_{2}$ values at rest than younger adults. Patients with chronic lung diseases may tolerate low levels of $\mathrm{SaO}_{2}$ chronically. However, although chronically hypoxaemic patients may tolerate an abnormally low $\mathrm{SaO}_{2}$ at rest when in a clinically stable condition, these resting oxygen levels may not be adequate for tissue oxygenation during acute illness when the tissue oxygen demand may increase (eg, sepsis, trauma, pneumonia, head injury; see section 8).

Acute hypoxaemia with $\mathrm{SaO}_{2}<90 \%$ and sometimes $<80 \%$ is seen in many acute illnesses such as pneumonia and heart failure and it is likely that the clinical manifestations of hypoxaemia in illness would be similar to those of experimental hypoxaemia in hypobaric chambers (impaired mental function followed by loss of consciousness). However, the clinical manifestations of the illness itself make it difficult to identify 
which symptoms and signs are due to hypoxaemia. Pure hypoxaemia, as seen in hypobaric chambers and at altitude, does not seem to cause breathlessness in resting subjects.

Patients with chronic diseases such as COPD, lung fibrosis, neuromuscular disorders or congenital heart disease may routinely attend outpatient clinics with $\mathrm{SaO}_{2}$ levels well below $90 \%$ even at a time when their disease is stable. In an emergency a clinician who was not familiar with such a patient (when stable) might interpret the low saturation as having occurred acutely and aim to achieve an oxygen saturation that was well above the patient's usual oxygen saturation level. Many such patients would qualify for long-term oxygen therapy. The UK COPD guideline ${ }^{25}$ recommends a threshold of $7.3 \mathrm{kPa}(55 \mathrm{~mm} \mathrm{Hg})$ below which most patients with COPD will benefit from long-term oxygen therapy (equivalent to a $\mathrm{SaO}_{2}$ of about $88-89 \%$ ) and an arterial oxygen tension $\left(\mathrm{PaO}_{2}\right)$ threshold below $8.0 \mathrm{kPa}(60 \mathrm{~mm} \mathrm{Hg})$ for patients with established cor pulmonale and some other subgroups.

- Many patients with chronic lung disease, congenital cyanotic heart disease or chronic neuromuscular conditions have oxygen saturations substantially below the normal range, even when clinically stable. [Evidence III]

\subsubsection{Variation in oxygen saturation during sleep}

Healthy subjects in all age groups have greater variation in $\mathrm{SaO}_{2}$ when sleeping than while awake. A study of 330 people referred to a sleep laboratory with normal results of overnight polysomnography (patients with cranial facial or neurological abnormalities or previously diagnosed pulmonary disease were excluded) showed that desaturation routinely occurred with a mean (SD) minimum $\mathrm{SaO}_{2}$ or "nadir" of 90.4 (3.1)\% during the night (2SD range 84.2-96.6\%). ${ }^{26}$ The mean (SD) overnight $\mathrm{SaO}_{2}$ "nadir" was 89.3 (2.8)\% for subjects aged $>60$ years. ${ }^{26}$ In this study subjects aged $20-30$ years spent $10 \%$ of the night with $\mathrm{SaO}_{2}$ levels below $94.8 \%$ and half the night below $96.3 \%$, and those aged $>60$ years spent $10 \%$ of the night below $92.8 \%$ and half the night below $95.1 \%$. Furthermore, the authors of this study excluded obese patients with any features of sleep apnoea or hypopnoea because these patients are known to desaturate to very low levels during sleep (often below 70\%). The variation in $\mathrm{SaO}_{2}$ during sleep is exaggerated by alcohol and by sedative drugs. This makes it difficult to evaluate a "spot reading" of $\mathrm{SaO}_{2}$ on a sleeping subject. It is suggested that $\mathrm{SaO}_{2}$ measurements of sleeping subjects should be interpreted with caution and ideally observed for a few minutes to see if the subject has got sustained hypoxaemia or just a transient normal "nocturnal dip".

\section{- All subjects have transient dips in oxygen saturation at night with a mean nadir of $90.4 \%$ (2SD range 84.2-96.6\%) in healthy subjects in all age groups. [Evidence III]}

\subsubsection{Normal range for carbon dioxide tension $\left(\mathrm{PaCO}_{2}\right)$ in the blood} The reference range for arterial carbon dioxide tension $\left(\mathrm{PaCO}_{2}\right)$ is approximately $4.6-6.1 \mathrm{kPa}(34-46 \mathrm{~mm} \mathrm{Hg})$ for healthy adult men aged $18-38$ years. ${ }^{27}$ Although this study was undertaken in 1948 , it is consistent with the clinical experience of the guideline group members and with most modern reference values for $\mathrm{PaCO}_{2}$. Although different laboratories and textbooks give slightly different reference values, all are within $0.2 \mathrm{kPa}$ of the above reference range. Any value of $\mathrm{PaCO}_{2}$ of $>6.1 \mathrm{kPa}$ (45 $\mathrm{mm} \mathrm{Hg}$ ) should be considered abnormal, but values up to $6.7 \mathrm{kPa}(50 \mathrm{~mm} \mathrm{Hg})$ may be obtained by breath-holding.
3.2 Definitions of hypoxaemia, hypoxia, type 1 respiratory failure and hyperoxia

\section{Hypoxaemia}

Hypoxaemia refers to low oxygen tension or partial pressure of oxygen $\left(\mathrm{PaO}_{2}\right)$ in the blood. For practical reasons, hypoxaemia can also be measured in relation to oxyhaemoglobin saturation. In adults the normal range is influenced by age and comorbidity and the normal ranges for healthy adults are given in section 3.1.1. The precise level at which a patient becomes hypoxaemic is debatable. One could argue that any saturation below the lower limit of normal constitutes hypoxaemia. Various authors have defined hypoxaemia as $\mathrm{SaO}_{2}$ of (1) $<94 \%$; (2) <92\%; (3) <90\%; or (4) $\mathrm{PaO}_{2}<60 \mathrm{~mm} \mathrm{Hg}$ or $8 \mathrm{kPa}{ }^{2}{ }^{28-30}$ Most authors who have studied this area have defined hypoxaemia as $\mathrm{PaO}_{2}<60 \mathrm{~mm} \mathrm{Hg}\left(8 \mathrm{kPa}\right.$ ) or $\mathrm{SaO}_{2}$ $<90 \%$. ${ }^{31}$ There is no known risk of hypoxic tissue injury above this level and many guidelines on critical care set $90 \%$ as the minimum below which $\mathrm{SaO}_{2}$ should not be allowed to fall. ${ }^{23}{ }^{24}$

\section{Type 1 respiratory failure}

Type 1 respiratory failure is most widely defined as $\mathrm{PaO}_{2}<8 \mathrm{kPa}$ or $60 \mathrm{~mm} \mathrm{Hg}$ (equivalent to $\mathrm{SaO}_{2}$ of approximately $90 \%$ ) with a normal or low $\mathrm{PaCO}_{2}$ level. ${ }^{32}$

\section{Hypoxia}

The term hypoxia is less specific and refers to lack of oxygen in a particular compartment (eg, alveolar or tissue hypoxia). Tissue hypoxia may result from four main causes (see below). It should be noted that the first two causes-hypoxaemia and anaemiado not always result in tissue hypoxia as oxygen delivery to tissues can be augmented in other ways such as increasing cardiac output.

\section{Hypoxaemic hypoxia}

Hypoxaemic hypoxia (sometimes also referred to as hypoxic hypoxia) is present when the oxygen content in the blood is low due to reduced partial pressure of oxygen. This occurs naturally at altitude and in many diseases such as emphysema which impair the efficiency of gas exchange in the lungs.

\section{Anaemic hypoxia}

Anaemic hypoxia results from a reduced level of haemoglobin available for oxygen transport. Although the patient may not be hypoxaemic (with a normal $\mathrm{PaO}_{2}$ and oxygen saturation measured by oximetry $\left(\mathrm{SpO}_{2}\right)$ ), the reduced oxygen content of the blood may lead to tissue hypoxia. Carbon monoxide poisoning may also produce a form of anaemic hypoxia by impairing the ability of haemoglobin to bind oxygen, thereby reducing oxygen-carrying capacity.

\section{Stagnant hypoxia}

Stagnant hypoxia is a low level of oxygen in the tissues due to inadequate blood flow (either globally or regionally). This condition may occur in the extremities if a person is exposed to cold temperatures for prolonged periods of time and it is the cause of gangrene in tissue that is deprived of blood in severe peripheral vascular disease. Stagnant hypoxia may occur in low cardiac output states.

\section{Histotoxic hypoxia}

Histotoxic hypoxia is an inability of the tissues to use oxygen due to interruption of normal cellular metabolism. The best known example of this occurs during cyanide poisoning which 
impairs cytochrome function. It is increasingly thought that mitochondrial dysfunction may lead to decreased oxygen utilisation in sepsis despite adequate oxygen delivery. This has also been termed "cytopathic dysoxia". 33

\section{Hyperoxia and hyperoxaemia}

Hyperoxia and hyperoxaemia are the counterparts to the above terms and in this guideline refer to high oxygen content in the blood and high oxygen tension in the blood, respectively. As stated above, for practical purposes the oxygen tension in the blood is often measured as oxyhaemoglobin saturation. Furthermore, this guideline is centred on providing target saturations for various conditions, but it should be noted that above a $\mathrm{PaO}_{2}$ of approximately $16 \mathrm{kPa}(120 \mathrm{~mm} \mathrm{Hg})$ the oxyhaemoglobin saturation will obviously not change from $100 \%$, yet the effects of further increases in $\mathrm{PaO}_{2}$ may be important in certain conditions such as COPD. This is discussed in further detail in sections 5 and 6 .

\subsection{Definition of hypercapnia and type 2 respiratory failure}

Hypercapnia is present when the $\mathrm{PaCO}_{2}$ is above the normal range of $4.6-6.1 \mathrm{kPa}(34-46 \mathrm{~mm} \mathrm{Hg})$ and patients with hypercapnia are said to have type 2 respiratory failure even if the oxygen saturation is in the normal range. ${ }^{32}$

\subsection{Definition of acidosis (respiratory acidosis and metabolic acidosis)}

\section{Acidosis}

Acidity in any fluid is determined by the concentration of hydrogen ions $\left[\mathrm{H}^{+}\right]$, and this is normally regulated between 35 and $45 \mathrm{nmol} / \mathrm{l}$. Acidity is more often expressed in terms of $\mathrm{pH}$ where $\mathrm{pH}=-\log _{10}\left[\mathrm{H}^{+}\right]$. The normal $\mathrm{pH}$ range of the blood in humans is between 7.35 and 7.45 units. Acidosis is defined as a $\mathrm{pH}<7.35\left(\left[\mathrm{H}^{+}\right]>45 \mathrm{nmol} / \mathrm{l}\right)$ and alkalosis is defined as a $\mathrm{pH}$ $>7.45\left(\left[\mathrm{H}^{+}\right]<35 \mathrm{nmol} / \mathrm{l}\right)$. Acidosis can be caused by respiratory or metabolic disorders.

\section{Respiratory acidosis}

Carbon dioxide $\left(\mathrm{CO}_{2}\right)$ can combine with water $\left(\mathrm{H}_{2} \mathrm{O}\right)$ to form carbonic acid $\left(\mathrm{H}_{2} \mathrm{CO}_{3}\right)$ in the blood which, in turn, dissociates to bicarbonate $\left(\mathrm{HCO}_{3}{ }^{-}\right)$and a hydrogen ion $\left(\mathrm{H}^{+}\right)$. Acute respiratory acidosis occurs if the $\mathrm{pH}$ of the blood falls below $7.35\left(\left[\mathrm{H}^{+}\right]>45 \mathrm{nmol} / \mathrm{l}\right)$ in the presence of a raised $\mathrm{CO}_{2}$ level.

If respiratory acidosis has been present for more than a few hours the kidney retains bicarbonate to buffer the acidity of the blood and, over hours to days, this may be sufficient to produce a normal $\mathrm{pH}$. This situation (high $\mathrm{PaCO}_{2}$ with high bicarbonate and normal pH) is known as "compensated respiratory acidosis". This situation is common in patients with chronic severe but stable COPD, but they may have an additional acute rise in $\mathrm{PaCO}_{2}$ during an acute exacerbation giving rise to "acute on chronic" respiratory acidosis despite their high bicarbonate level. This happens because the bicarbonate level was equilibrated with the previous $\mathrm{CO}_{2}$ level and is insufficient to buffer the sudden further increase in $\mathrm{CO}_{2}$ level that may occur during an exacerbation of COPD. Respiratory acidosis is common in clinical practice. Plant and colleagues showed that about $20 \%$ of patients with acute exacerbations of COPD requiring hospital admission have respiratory acidosis. ${ }^{34}$

\section{Metabolic acidosis}

This can be caused by failure to excrete acid produced by the body's normal metabolic processes (eg, during renal failure) or by increased production of acid from abnormal metabolic conditions such as diabetic ketoacidosis. Alternatively, it may result from direct loss of bicarbonate from the kidney or gut (eg, during chronic diarrhoea). In all forms of metabolic acidosis there is a low blood bicarbonate level, either due to loss of bicarbonate or due to buffering of excess acid by bicarbonate which is excreted as $\mathrm{CO}_{2}$. A common cause of metabolic acidosis is lactic acidosis caused by tissue hypoxia. This may result from decreased oxygen delivery such as occurs in hypoxaemia, or low cardiac output states or conditions such as sepsis where oxygen consumption is impaired in the face of adequate oxygen delivery. In health, metabolic acidosis will occur at peak exercise where oxygen delivery is insufficient to meet demand.

\section{SECTION 4: GENERAL BLOOD GAS PHYSIOLOGY}

A full understanding of blood gas physiology in the body requires a detailed understanding of the anatomy, physiology and biochemistry of respiration and gas exchange. It is recognised that most readers of this guideline may not have had full training in all of these specialties, so this physiology section contains a brief overview of basic principles for the nonspecialist reader (section 4) followed by a more detailed overview of the pathophysiology of oxygen for the expert reader (section 5 ). The rationale for targeted oxygen therapy is discussed in detail in section 6 .

\subsection{Oxygen physiology}

Oxygen is the main "fuel" of the cells in mammalian bodies and it is essential for humans to maintain a safe level of oxygen in the bloodstream. Most of the oxygen carried in the blood is bound to an oxygen-carrying protein in red blood cells called haemoglobin. Oxygen itself does not dissolve easily in blood so only a small amount is carried dissolved in the bloodstream. As there is a fixed amount of haemoglobin circulating in the blood, the amount of oxygen carried in the blood is often expressed in terms of how saturated with oxygen the circulating haemoglobin is. This is what is meant by "oxygen saturation level". If this is measured directly from an arterial blood sample, it is called the $\mathrm{SaO}_{2}$. If the measurement is calculated from a pulse oximeter it is called the $\mathrm{SpO}_{2}$. Alternatively, one can measure the oxygen tension of the blood $\left(\mathrm{PaO}_{2}\right)$, known as the "partial pressure of oxygen" in the blood. This measurement can be expressed in kilopascals $(\mathrm{kPa})$ (normal range 12.0-14.6 kPa) or in millimetres of mercury (normal range $90-110 \mathrm{~mm} \mathrm{Hg}$ for young adults). ${ }^{11}$

The normal $\mathrm{SaO}_{2}$ in healthy adults at sea level is maintained within a narrow range of about $95-98 \%$ as discussed in section 3.1 above. This means that almost all of the oxygen-carrying capacity of haemoglobin in the blood is used when the $\mathrm{SaO}_{2}$ is in the normal range. Therefore, giving supplementary oxygen to a healthy young person will increase the saturation level only slightly from about $97 \%$ to $99 \%$ or a maximum of $100 \%$, thus producing only a very small increase in amount of oxygen made available to the tissues.

Sudden exposure to low $\mathrm{SaO}_{2}$ levels (below about $80 \%$ ) can cause impaired mental functioning even in healthy subjects. The brain is the most sensitive organ to the adverse effects of hypoxia, but it is possible that other organs in patients with critical illness may be vulnerable to the risk of hypoxic tissue injury at oxygen levels above this range. Most experts emphasise the importance of keeping the $\mathrm{SaO}_{2}$ above $90 \%$ for the majority of acutely ill patients. ${ }^{21-24}$ The present guideline suggests a desirable target saturation range of $94-98 \%$. This 
range mirrors the normal range for UK adults with a wide margin of safety above the $90 \%$ threshold which is mentioned above.

Oxygen passes from inspired air in the lungs into the bloodstream and is delivered to the tissues. If oxygen levels fall in the blood, this is sensed by receptors in the carotid body (connected to the carotid artery in the neck) and ventilation is stimulated to increase the amount of oxygen coming into the lung and therefore the blood. The lung has the ability to divert blood flow away from areas which are poorly ventilated, so that blood returning from the body can be replenished with oxygen and can also clear carbon dioxide. This occurs through a process called "hypoxic vasoconstriction" whereby localised low oxygen levels in the lung airspaces cause constriction of feeding blood vessels, therefore diverting blood to areas of the lung with more normal oxygen levels.

If the oxygen-carrying capacity of the blood is low as, for example, in anaemia, this is detected by the kidneys which produce a hormone, erythropoietin, to stimulate red blood cell production. As one of the goals of the circulation is to deliver oxygen to the tissues of the body, the heart also responds to low oxygen levels by increasing its output, so increasing "oxygen delivery".

Hypoxaemia, low $\mathrm{PaO}_{2}$, can be caused by a number of mechanisms. The most common form of hypoxaemia occurs when there is sufficient oxygen-carrying capacity (in patients with a normal level of haemoglobin) but insufficient oxygen taken up in the lungs. This can be the result of poor aeration of areas of lung or due to abnormalities of gas exchange within the lung during serious illnesses such as pneumonia. This form of hypoxaemia is the easiest to treat with oxygen therapy. Oxygen therapy is less effective in other causes of hypoxaemia including anaemia where there is a low carrying capacity or where the carrying capacity of haemoglobin has been reduced by a toxic substance because oxygen availability is not the limiting feature in these conditions. For example, carbon monoxide blocks oxygen binding to haemoglobin despite having a normal level of oxygen in the lungs and in the blood.

\subsection{Carbon dioxide physiology}

Carbon dioxide is a product of the body's metabolism. It is cleared from the body by being transferred from the bloodstream into the alveoli in the lungs and then exhaled from the lungs. In a similar way to oxygen, carbon dioxide levels in the blood are controlled by chemical sensors (both in the carotid body and brainstem).

Carbon dioxide is highly soluble in the blood and is carried in three forms: bicarbonate (70\%), dissolved carbon dioxide $(10 \%)$ and bound to haemoglobin (20\%). As carbon dioxide carriage is not limited by a carrier molecule such as haemoglobin, it is not expressed as a saturation. Because its carriage is approximately proportional to the partial pressure (gas tension) of carbon dioxide in the blood within the physiological range, carbon dioxide carriage is usually expressed in terms of its partial pressure. The normal range is $4.6-6.1 \mathrm{kPa}$ or $34-46 \mathrm{~mm} \mathrm{Hg}$.

Increased levels of carbon dioxide will stimulate ventilation, thus increasing clearance from the lungs and therefore from the bloodstream. However, this mechanism is less effective in some respiratory diseases such as COPD where increased airway resistance and respiratory muscle weakness can restrict this response. Hypercapnia will occur when there is decreased ventilation for any reason. Safe elimination of carbon dioxide is as important to the body as the intake of oxygen.
Too little oxygen can give rise to organ failure but too much oxygen can also be harmful in some situations, especially to some vulnerable patients with COPD, chest wall deformities or muscle weakness. About a quarter of patients with acute flare-ups of COPD are at risk of carbon dioxide retention if they are given an excessively high dose of oxygen. If high concentrations of oxygen are given to these patients, the oxygen level in the blood will rise but the level of carbon dioxide will also rise and this can cause acidosis with subsequent organ dysfunction and, when severe, coma. In the past it was thought that the main problem was that these patients were dependent on the stimulus of a low blood oxygen level — called "hypoxic drive" to stimulate breathing. It was thought that giving oxygen would cause a rise in the carbon dioxide level by simply reducing the stimulus to breathe due to "lack of hypoxic drive". It is now known that the mechanisms for carbon dioxide retention in some patients are much more complex than this simple model suggested. Much of the rise in carbon dioxide which occurs during high-dose oxygen therapy is due to deterioration in the matching of blood flow and gas flow in the lungs. This can be avoided by giving controlled lower concentration oxygen therapy to vulnerable patients (see table 3).

\subsection{Concept of target oxygen saturation $\left(\mathrm{SaO}_{2}\right)$ ranges}

One might ask why one should not aim for an $\mathrm{SaO}_{2}$ of $100 \%$ (hyperoxaemia) in all acutely ill patients (and some clinicians took this view in the past). This policy would clearly be risky for vulnerable patients with COPD and chest wall problems, but it could also harm other patients in a variety of ways. The more controversial risks of hyperoxaemia include coronary and cerebral vasoconstriction and decreased cardiac output. Although these physiological effects are well documented, their significance in clinical practice is almost unknown owing to a lack of clinical trials of oxygen therapy.

High oxygen concentrations lead to an increase in reactive oxygen species which may cause tissue damage and may be responsible for some of the detrimental effects observed with high-flow oxygen in myocardial infarction and stroke. It is recognised that very high inhaled oxygen levels can give rise to partial collapse of some lung units, a condition known as "absorption atelectasis". There is also the potential concern that a high oxygen saturation produced by high concentration oxygen therapy could mask a major deterioration in the patient's clinical condition causing dangerous delays in treatment. An example of this is a patient who has taken an opiate overdose which has produced respiratory depression and the patient is underbreathing. If the patient is given excessive oxygen therapy, high or normal oxygen saturations may be recorded at a time when the carbon dioxide levels are dangerously high. The high oxygen saturation could mask the real situation and give the health professionals a false sense of confidence.

As alluded to above, acutely raised carbon dioxide levels can be dangerous. In acute circumstances where carbon dioxide levels have risen rapidly, the kidneys are unable to compensate for the consequent increased acid load. There are good data to show that the lower the $\mathrm{pH}$ of the blood, the higher the risk of intubation or death in patients with exacerbations of COPD. ${ }^{34}$

The purpose of oxygen therapy is to increase oxygen delivery to tissues, not just to increase oxygen carried by the blood. It must therefore be remembered that there may be other physiological disturbances that need correcting to increase oxygen delivery such as low cardiac output and severe anaemia. For example, improving these factors will improve oxygen 


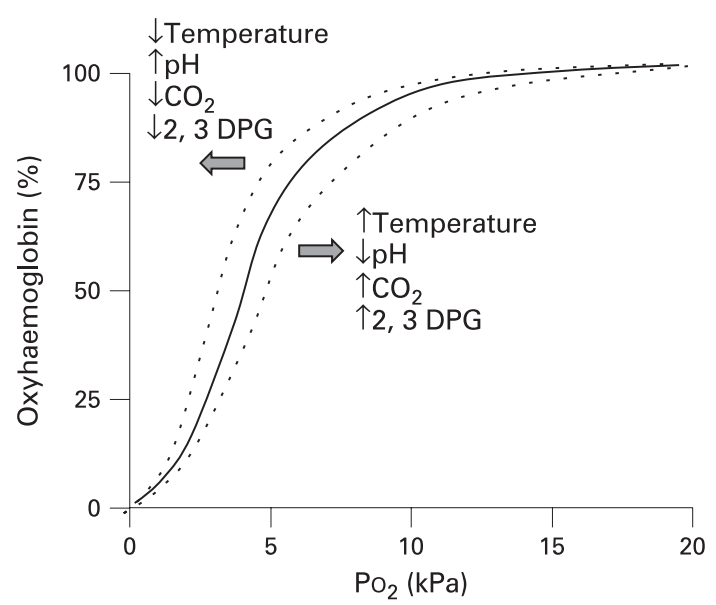

Figure 3 Oxygen dissociation curve with the Bohr effect. 2, 3 DPG, 2, 3 diphosphoglycerate; $\mathrm{Po}_{2}$, oxygen tension.

delivery much more than administering oxygen to a patient with a saturation of $90 \%$ which, at most, will produce a $10 \%$ rise in delivery. In addition to optimising oxygen delivery from the lungs to the tissues, it is important also to treat problems that might impair delivery of oxygen to the lungs themselves such as upper airway obstruction, bronchoconstriction and pulmonary oedema (remember the "ABC" of resuscitation airway, breathing, circulation).

There is uncertainty about defining the ideal target saturation and this is one of the core debates in oxygen therapy. This uncertainty is largely due to a lack of evidence from clinical trials. In some specific disease areas such as COPD there are good data to inform the ideal target saturation and these will be covered in sections 8 and 9 . In the general population without a specific indication for running high or low saturations, historically there has been a tendency to apply oxygen therapy even when saturations are in the "normal" range. There are no data to support this practice in common conditions such as ischaemic heart disease or stroke and, indeed, some studies show harm. It should be reassuring that supraphysiological levels of oxygen delivery are not required in critical illness unless specifically indicated (eg, carbon monoxide poisoning). The

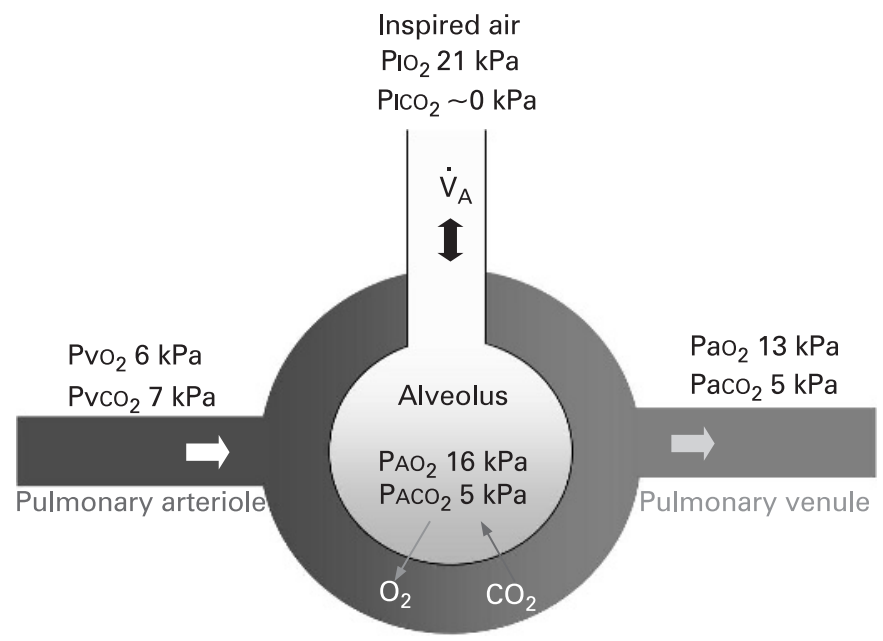

Figure 4 Alveolar-capillary unit. $\mathrm{PaCO}_{2}, \mathrm{PaO}_{2}$, arterial carbon dioxide and oxygen tensions; $\mathrm{PACO}_{2}, \mathrm{PAO}_{2}$, alveolar carbon dioxide and oxygen tensions; $\mathrm{PICO}_{2}, \mathrm{PlO}_{2}$, inspired carbon dioxide and oxygen tensions; $\mathrm{PvCO}_{2}$, $\mathrm{PvO}_{2}$, venous carbon dioxide and oxygen tensions. consensus among the members of the guideline group is that one should aim for a normal or near-normal $\mathrm{SaO}_{2}$ range of 94 $98 \%$ for acutely ill patients except those at risk of hypercapnic respiratory failure (see recommendations $1-5$ in section 6 of this guideline).

\section{SECTION 5: ADVANCED BLOOD GAS PHYSIOLOGY AND PATHOPHYSIOLOGY AND PHYSIOLOGY OF OXYGEN THERAPY}

Many of the issues discussed in this section are of a technical nature and may not be easily comprehensible to the general reader. However, recommendations $1-5$ in section 6 of the guideline will follow logically from this section and from the brief overview of oxygen physiology in section 4 .

The neurocardiopulmonary axis is designed to optimise global oxygen delivery and carbon dioxide clearance and the local tissue vascular beds are responsible for the distribution of blood flow.

Oxygen delivery $\left(\mathrm{DO}_{2}\right)$ is expressed by the equation:

$\mathrm{DO}_{2}=\mathrm{CaO}_{2} \times 0$

where $\mathrm{CaO}_{2}$ is the oxygen content of the arterial blood and $\mathrm{Q}$ is the cardiac output. $\mathrm{CaO}_{2}$ is the sum of oxygen dissolved in the blood and the amount of oxygen carried by haemoglobin. The solubility of oxygen in the blood is very low and therefore $\mathrm{CaO}_{2}$ is largely determined by the total amount of haemoglobin and the proportion which is bound by oxygen, namely saturation. The relationship between haemoglobin $\mathrm{SaO}_{2}$ and $\mathrm{PaO}_{2}$ is shown in fig 3 and table 8 . In health and disease, haemoglobin saturation is also influenced by other factors such as $\mathrm{pH}, \mathrm{PCO}_{2}$, temperature and 2,3 diphosphoglycerate (Bohr effect; fig 3 and section 5.1.3). Consequently, there is not an exact relationship between $\mathrm{SaO}_{2}$ and $\mathrm{PaO}_{2}$ but table 8 gives approximate equivalents.

\subsection{Regulation of blood oxygen content $\left(\mathrm{CaO}_{2}\right)$}

Figure 4 shows the level of oxygen and carbon dioxide in the pulmonary artery, in the alveolus and room air and in the pulmonary venous circulation which leads directly to the arterial circulation. The $\mathrm{PaO}_{2}$ of mixed systemic venous blood rises markedly from a low level in the pulmonary artery (about $6 \mathrm{kPa}$ or $45 \mathrm{~mm} \mathrm{Hg}$ ) to about $16 \mathrm{kPa}(120 \mathrm{~mm} \mathrm{Hg})$ by the end of the pulmonary capillary. However, because the lung is not homogeneously made up of alveolar capillary units that are matched for perfusion and ventilation, the $\mathrm{PaO}_{2}$ in the larger pulmonary veins is lower $(13 \mathrm{kPa}, 100 \mathrm{~mm} \mathrm{Hg})$. This is explained in more detail below. The gradient of carbon dioxide is much more gradual, falling from about $7 \mathrm{kPa}(52 \mathrm{~mm} \mathrm{Hg})$ in the venous system and pulmonary artery to about $5 \mathrm{kPa}$ $(37 \mathrm{~mm} \mathrm{Hg}$ ) in the pulmonary vein and in the arterial system.

\subsubsection{Arterial oxygen tension $\left(\mathrm{PaO}_{2}\right)$}

The pulmonary vasculature maximises $\mathrm{PaO}_{2}$ by ensuring that the well ventilated areas of the lung receive most of the pulmonary blood flow, a process called ventilation/perfusion $(\mathrm{V} / \mathrm{Q})$ matching. This is largely achieved through a process called hypoxic pulmonary vasoconstriction (HPV). ${ }^{35}$ The pulmonary circulation is unique in this regard compared with all the other vascular beds in the body which dilate in response to hypoxia. In poorly ventilated areas of lung the precapillary pulmonary arterioles constrict in response to sensing low alveolar $\mathrm{PO}_{2}\left(\mathrm{PAO}_{2}\right)$. This is a compensating process and, despite it, some deoxygenated blood may still leave poorly ventilated alveolar capillary units. Deoxygenated blood leaving poorly ventilated alveolar capillary units cannot be compensated for by 


\section{Example 1}

- Before oxygen therapy assume $50 \%$ of pulmonary flow is passing through an area of low $\mathrm{V} / 0$ and that the pulmonary venous oxyhaemoglobin saturation $\left(\mathrm{Spvo}_{2}\right)$ from this compartment is $80 \%$ (ie, just above mixed venous $\mathrm{SO}_{2}$ ). The other $50 \%$ is passing through an area of matched $\mathrm{V} / \mathrm{Q}$, resulting in a $\mathrm{Spvo}_{2}$ of $97 \%$. The final mixed $\mathrm{Spvo}_{2}$ will be $88.5 \%$.

- Following maximal oxygen therapy, assuming no change in flow as a result of release of HPV, $\mathrm{SpvO}_{2}$ from the low V/O compartment rises to $85 \%$ and $\mathrm{Spvo}_{2}$ from the matched compartment rises to a maximum of $100 \%$. The resulting mixed $\mathrm{Spvo}_{2}$ will now only be $92.5 \%$. This has occurred because fully saturated blood cannot increase its oxygen content beyond full saturation despite an increase in $\mathrm{PO}_{2}$, apart from the minimal contribution from dissolved oxygen; ie, the relationship between $\mathrm{PO}_{2}$ and oxyhaemoglobin saturation/ blood oxygen content is not linear.

mixing with blood from well ventilated units as the relationship between $\mathrm{PaO}_{2}$ and $\mathrm{CaO}_{2}$ is not linear. This physiological phenomenon is often not fully appreciated and therefore is worth a theoretical worked example (see box).

A much less studied phenomenon that regulates $\mathrm{V} / \mathrm{O}$ matching is hypoxic bronchodilation. This effect increases ventilation to poorly ventilated areas of the lung. ${ }^{36}$

If $\mathrm{PaO}_{2}$ falls, the peripheral chemoreceptors in the carotid body drive an increase in ventilation to increase $\mathrm{PaO}_{2} \cdot{ }^{37}$ This will not increase $\mathrm{PaO}_{2}$ leaving already well ventilated units, but will increase $\mathrm{PaO}_{2}$ leaving less well ventilated alveolar units by increasing $\mathrm{PAO}_{2}$ in these units. Although the ventilatory response to $\mathrm{SaO}_{2}$, and therefore $\mathrm{CaO}_{2}$, is linear (fig 5), the carotid body senses $\mathrm{PaO}_{2}$ and not $\mathrm{CaO}_{2}$. This prevents excessive ventilation in response to anaemia which would be ineffective in increasing $\mathrm{CaO}_{2}$. The peripheral chemoreceptors are able to do this because the very high ratio of $\mathrm{DO}_{2}$ to oxygen consumption of the carotid body means that the tissue $\mathrm{PO}_{2}$ in the carotid body continues to reflect $\mathrm{PaO}_{2}$ and will not fall even in the presence of anaemic hypoxia. ${ }^{38}{ }^{39}$

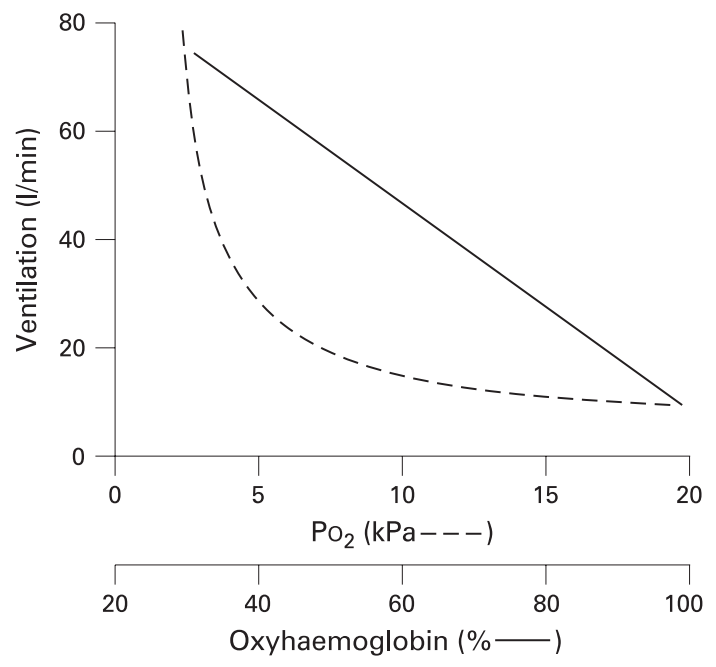

Figure 5 Ventilatory response to hypoxaemia. The relationship is inversely linear when plotted against oxyhaemoglobin (solid line) saturation but inversely exponential when plotted against arterial oxygen tension $\left(\mathrm{PO}_{2}\right)$ (dashed line).

\subsubsection{Haematocrit}

Erythropoiesis is controlled by a negative feedback system involving erythropoietin. By contrast with the carotid bodies, the peritubular cells in the kidney are well suited to sensing oxygen delivery as oxygen extraction is relatively high compared with oxygen delivery. ${ }^{40}{ }^{41}$ Although oxygen delivery to the kidneys as a whole organ is high due to high renal blood flow, $\mathrm{DO}_{2}$ is reduced to the renal medulla as oxygen can pass from arterioles to the post-capillary venous system by shunt diffusion due to the parallel organisation of arterial and venous systems. ${ }^{42}$ Consequently, the peritubular cellular $\mathrm{Po}_{2}$ is low. It falls to even lower levels following reductions in $\mathrm{Do}_{2}$ either as a result of hypoxaemia or low haematocrit.

\subsubsection{The Bohr effect}

The oxygen-carrying capacity of haemoglobin is regulated in response to other metabolic factors to increase the efficiency of oxygen pick-up and delivery. ${ }^{43}$ Acidosis and hypercapnia shift the oxygen dissociation curve to the right (fig 3), thus favouring the dissociation of oxygen from haemoglobin in metabolically active tissues. The converse would hold true for the lungs where lower carbon dioxide levels favour oxygen loading of haemoglobin. Chronic hypoxaemia increases 2,3-diphosphoglycerate (2,3-DPG) in erythrocytes, shifting the dissociation curve to the right and therefore increasing oxygen delivery to the tissues.

\subsubsection{Regulation of $\mathrm{Do}_{2}$ (oxygen delivery from the lungs to the tissues)}

Acutely, the cardiovascular effects of hypoxaemia will tend to counter the impact of lower $\mathrm{CaO}_{2}$ on $\mathrm{DO}_{2}$ by increasing cardiac output through increased heart rate and myocardial contractility and by decreasing afterload by reducing systemic vascular resistance. ${ }^{44}{ }^{45}$ Anaemic hypoxia is sensed in the aortic body, presumably owing to lower perfusion relative to oxygen consumption. Consequently, the aortic body can act as a sensor of reduced oxygen delivery as a result of either low oxygen tension or low haematocrit (unlike the carotid body). ${ }^{38}$

At local tissue level, oxygen delivery can be adjusted to changes in local oxygen consumption. For example, exercising skeletal muscle receives a greater proportion of total cardiac output than resting skeletal muscle. This relates in part to hypoxaemia recruiting a larger proportion of the capillary bed by the relaxation of pericytes, and also through arteriolar vasodilatation. $^{46}$

\subsection{Pathophysiology of hypoxia and hyperoxia}

Hypoxia may result from a number of different diseases discussed in section 8 of this guideline. In each case one or more of the following pathophysiological mechanisms may apply:

- Hypoxaemic hypoxia

- Other mechanisms of hypoxia

- Hyperoxia

\subsubsection{Hypoxaemic hypoxia (see definition in section 3.1.2)}

Hypoxaemic hypoxia in blood leaving an alveolar capillary unit in the lung may be induced by alveolar hypoxia or incomplete gas exchange. The alveolar gas equation calculates the oxygen level in the alveolus using the following formula:

$\mathrm{PAO}_{2} \approx \mathrm{PIO}_{2}-\mathrm{PACO}_{2} / \mathrm{RER}$

where $\mathrm{PAO}_{2}$ and $\mathrm{PACO}_{2}$ represent alveolar levels of oxygen and carbon dioxide, RER is the respiratory exchange ratio or the ratio of carbon dioxide production to oxygen consumption and 
inspired $\mathrm{PO}_{2}\left(\mathrm{PIO}_{2}\right)=\mathrm{FIO}_{2} \times($ barometric pressure $[100 \mathrm{kPa}$, $750 \mathrm{~mm} \mathrm{Hg}$ - water vapour pressure [ $\sim 6 \mathrm{kPa}, 45 \mathrm{~mm} \mathrm{Hg}]$ ).

Considering this equation, alveolar hypoxia can be induced by decreased $\mathrm{PIO}_{2}$ or increased $\mathrm{PACO}_{2}$. If an alveolar capillary unit is relatively underventilated for its degree of perfusion (low V/O ratio), $\mathrm{PACO}_{2}$ will rise due to inadequate clearance and thus $\mathrm{PAO}_{2}$ will fall. This may happen for a number of reasons such as increased dead space ventilation during the non-fatiguing pattern of shallow respiration in respiratory failure or abnormal lung mechanics in advanced COPD. In diseases that cause global hypoventilation such as respiratory muscle weakness, effectively all areas of lung have low $\mathrm{V} / \mathrm{O}$ ratios and this explains the hypercapnia and hypoxaemia associated with these conditions.

An extreme form of low $\mathrm{V} / \mathrm{O}$ pathophysiology occurs in intrapulmonary and extrapulmonary shunt where no gas exchange occurs at all. An example of intrapulmonary shunt is when the airway to a lung segment is obstructed by mucus creating an area of lung tissue that is perfused but not ventilated, thus acting as a right-to-left shunt. An example of extrapulmonary shunt is a ventricular septal defect with rightto-left shunting in Eisenmenger's syndrome.

In health and at rest, oxygen has equilibrated across the alveolar capillary membrane one-third of the way along the length of the capillary. With increased thickness of this membrane, as in fibrotic lung disease, equilibration may take longer and an oxygen gradient may persist between the alveolus and blood at the end of the capillary. The overall effect of this when multiple alveolar capillary units are affected will lead to an increased alveolar-to-arterial (A-a) gradient. This is exacerbated during exercise, when capillary transit time decreases.

5.2.2 Other mechanisms of hypoxia (see definitions in section 3.1.2) Anaemia and carbon monoxide poisoning may result in "anaemic hypoxia" by reducing oxygen-carrying capacity. A low cardiac output state will reduce oxygen delivery even in the absence of hypoxaemia. Tissue hypoxia may develop in these circumstances and this is often termed "stagnant hypoxia".

\subsubsection{Hyperoxia}

Hyperoxia can be caused by hyperoxaemia and polycythaemia. Considering again the alveolar gas equation in the previous section, hyperoxaemia can only exist in the presence of high inspired $\mathrm{PO}_{2}$ or low $\mathrm{PACO}_{2}$ (resulting from hyperventilation). The term "hyperoxia" could technically be used to describe a patient with polycythaemia without hyperoxaemia, but most clinicians use the term only to describe situations in which the $\mathrm{PaO}_{2}$ is raised.

\subsection{Physiology of carbon dioxide}

\subsubsection{Normal carbon dioxide homeostasis}

Carbon dioxide is principally carried in the blood in three forms: carbon dioxide, bicarbonate and as a carbamino compound. ${ }^{47}$ In the normal physiological range of $4.5-6.0 \mathrm{kPa}(34-45 \mathrm{~mm} \mathrm{Hg})$ the relationship between $\mathrm{PaCO}_{2}$ and $\mathrm{CCO}_{2}$ (carbon dioxide content) can be considered linear (fig 6).

\subsubsection{Regulation of carbon dioxide}

$\mathrm{PaCO}_{2}$ is sensed at the peripheral ${ }^{37}$ and central chemoreceptors (in the medulla oblongata) by its effect on intracellular $\mathrm{pH}^{48}$ Consequently, the regulation of $\mathrm{PaCO}_{2}$ is intimately related to $\mathrm{pH}$ homeostasis (fig 7).

It is often not appreciated how $\mathrm{V} / \mathrm{O}$ matching relates to $\mathrm{PaCO}_{2}$. As discussed in section 5.2.1, alveolar capillary units with a low $\mathrm{V} / \mathrm{O}$ ratio have increased $\mathrm{PACO}_{2}$. Because of the high

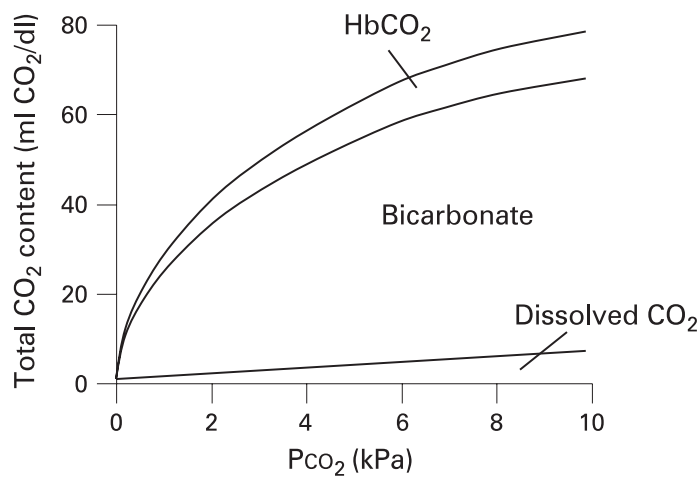

Figure 6 Total carbon dioxide dissociation curve. $\mathrm{PCO}_{2}$, carbon dioxide tension; $\mathrm{HbCO}_{2}$, carbamino haemoglobin.

solubility and diffusibility of carbon dioxide, there is little A-a gradient for carbon dioxide at the end of the capillary, so blood leaving low $\mathrm{V} / \mathrm{Q}$ alveolar capillary units has a high $\mathrm{PCO}_{2}$.

As described above, areas of low $\mathrm{V} / \mathrm{O}$ are usually minimised through hypoxic pulmonary vasoconstriction. It is also thought that a high $\mathrm{PCO}_{2}$ can cause pulmonary vasoconstriction, adding to the homeostatic mechanisms of the lung, matching perfusion to ventilation. ${ }^{49}{ }^{50}$ As the relationship between $\mathrm{PCO}_{2}$ and carbon dioxide dissolved in the blood is approximately linear over the physiological range (unlike oxygen), blood does not become saturated with carbon dioxide and therefore a high pulmonary venous $\mathrm{PCO}_{2}$ from low $\mathrm{V} / \mathrm{Q}$ areas can be partially balanced by a low pulmonary venous $\mathrm{PCO}_{2}$ from high $\mathrm{V} / \mathrm{Q}$ areas. Consequently, by increasing overall alveolar ventilation, the cardiopulmonary system is able to prevent hypercapnia despite significant $\mathrm{V} / \mathrm{Q}$ mismatch or shunt, unless respiratory mechanics are limiting.

As with the carriage of oxygen (Bohr effect), there is a reciprocal relationship between $\mathrm{PO}_{2}$ and carbon dioxide carriage. This is known as the Haldane effect. ${ }^{43}$ Deoxygenated haemoglobin has a higher carbon dioxide buffering capacity than oxygenated haemoglobin. This favours carbon dioxide pick-up in the systemic venous circulation and carbon dioxide offloading in the lungs.

Acutely, carbon dioxide acts as a sympathomimetic on the heart: it increases heart rate and stroke volume, increasing cardiac output. Peripherally it causes vasodilation, reducing systemic vascular resistance. Locally, carbon dioxide acts as a

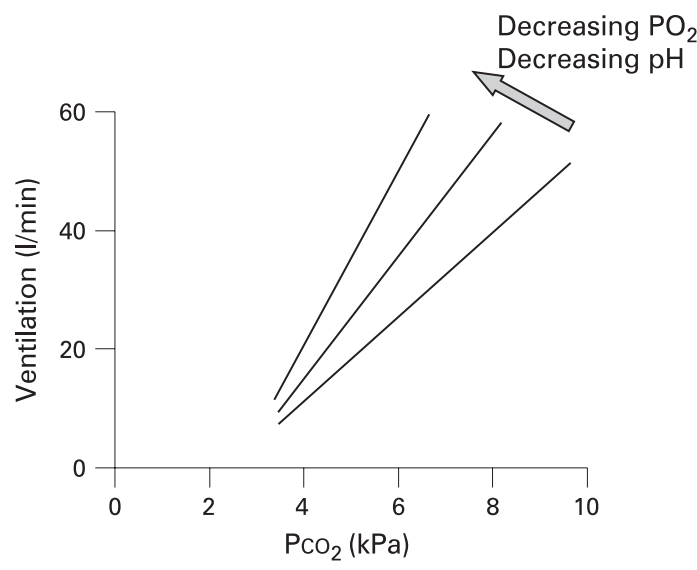

Figure 7 Effect of arterial carbon dioxide tension $\left(\mathrm{PaCO}_{2}\right)$ on ventilation with interaction of acidosis and hypoxaemia. 
vasodilator, thus diverting blood flow to tissues with high metabolic demand. The resulting physical signs of hypercapnia are described in section 7.2 .

\subsection{Pathophysiology of hypercapnia and hypocapnia}

\subsubsection{Mechanisms of hypercapnia and hypocapnia}

The mechanisms of hypercapnia are simpler than hypoxaemia and there are four possible causes: ${ }^{51}$

(1) Increased concentration of carbon dioxide in the inspired gas.

(2) Increased carbon dioxide production.

(3) Hypoventilation or ineffective ventilation.

(4) Increased dead space.

The mechanisms of hypercapnia in COPD (and other conditions predisposing to hypercapnic respiratory failure) are discussed in 6.3.1.

\section{Increased concentration of carbon dioxide in the inspired gas}

This iatrogenic cause of hypercapnia is uncommon but should be excluded at the outset in any patient unexpectedly found to be hypercapnic when breathing from, or being ventilated by, external equipment. The severity of hypercapnia due to rebreathing is limited by the rate at which the $\mathrm{PCO}_{2}$ can increase (no more than $0.4-0.8 \mathrm{kPa} / \mathrm{min}, 3-6 \mathrm{~mm} \mathrm{Hg} / \mathrm{min}$ ).

\section{Increased carbon dioxide production}

This is likely only to cause hypercapnia if the minute ventilation is fixed by artificial means and if carbon dioxide production is increased (eg, due to sepsis or increased work of breathing).

\section{Hypoventilation or ineffective ventilation}

Low alveolar minute ventilation is by far the most common cause of hypercapnia. In clinical practice, COPD is the most common disease to cause hypercapnia; the problem is secondary to alveolar hypoventilation rather than a reduced minute ventilation per se. Patients adopt a rapid shallow pattern of breathing during an acute exacerbation of COPD with the result that the ratio of dead space to tidal volume is increased with more ventilation therefore being "wasted". A rapid shallow pattern of breathing results in a bigger proportion of each breath being wasted because of the need to ventilate the anatomical dead space. Furthermore, during acute COPD exacerbations, V/ $\mathrm{Q}$ mismatch may lead to an increase in physiological dead space, exacerbating the problem further. It is important to note that this commonly occurs in the context of an apparent overall increase in minute ventilation. Alveolar hypoventilation due to a reduction in minute ventilation is seen following medullary respiratory centre depression by drugs, obstruction of a major airway or restriction of the lungs or chest wall or by respiratory muscle weakness, head injury, intracerebral haemorrhage or opioid narcosis.

\section{Increased dead space}

This would be most common in patients breathing through artificial apparatus which has been incorrectly configured. It can also be due to any cause of $\mathrm{V} / \mathrm{Q}$ mismatch in which the normal response to hypoxaemia (ie, to increase ventilation) is compromised because of lung disease. It is important to note therefore that hypercapnia sometimes may be seen in conditions more usually associated with hypocapnia (eg, pulmonary embolus, pneumonia) when it occurs in patients with lung disease and an increased physiological dead space. Although alveolar hypoventilation is the most common cause of hypercapnia, it is important to consider the other potential causes, particularly when patients are receiving assisted ventilation and an artificial breathing circuit is used.

\subsubsection{Hypoventilaton and hyperventilation}

Hypoventilation may be physiological-for example, in the face of a metabolic alkalosis. Pathological hypoventilation will occur either when the respiratory muscles are unable to ventilate the lungs sufficiently because they are pathologically weak or they are unable to overcome abnormal lung mechanics such as during an exacerbation of COPD. Reduced respiratory drive caused by drugs with sedative properties or by neurological injury will also produce hypoventilation.

Using the same physiological principles but in reverse, hyperventilation for any reason will produce hypocapnia. This may occur during pure hyperventilation during an anxiety attack or during physiological hyperventilation.

\subsection{Physiology of oxygen therapy}

Oxygen therapy increases $\mathrm{PAO}_{2}$ and is therefore only effective when alveolar capillary units have some functional ventilation. Oxygen therapy is ineffective if there is a pure shunt (such as pulmonary arteriovenous malformations) where mixed venous blood does not pass through an alveolar capillary unit. There will only be a small overall increase in $\mathrm{PaO}_{2}$ due to an increase in dissolved oxygen in the pulmonary venous blood from ventilated alveolar capillary units, which is small compared with the content of oxygen carried by haemoglobin. Despite this, there is good evidence that breath-hold times can be increased by breathing oxygen. ${ }^{52-54}$ One study found that the breath-hold time of 15 healthy subjects increased from $56 \mathrm{~s}$ after breathing air to $92 \mathrm{~s}$ after breathing 4 litres of nasal oxygen for $2 \mathrm{~min}$, and another study found that 31 healthy volunteers had an increase in breath-hold time from $32 \mathrm{~s}$ breathing air to $61 \mathrm{~s}$ after breathing oxygen whereas the breath-hold time of 29 patients with chronic pulmonary disease was $9 \mathrm{~s}$ compared with $22 \mathrm{~s}$ for a group of 29 similar patients after breathing oxygen. The same principles are used to preoxygenate patients before intubation during anaesthesia. It is thought that the additional breath-hold time is produced not by the marginal increase in blood oxygen levels but by the increased reservoir of oxygen in the lungs after breathing oxygen-enriched air.

In poorly ventilated units (ie, low $\mathrm{V} / \mathrm{O}$ ratio), $\mathrm{PAO}_{2}$ will be low. Increasing $\mathrm{FIO}_{2}$ will increase $\mathrm{PAO}_{2}$ and therefore $\mathrm{PaO}_{2}$. Hypoventilation disorders can be considered as lungs made up entirely of low $\mathrm{V} / \mathrm{O}$ units.

When there is diffusion limitation due to increased alveolar capillary membrane thickness such as in fibrotic lung disease, increasing $\mathrm{PAO}_{2}$ will augment the rate of diffusion across the alveolar capillary membrane by increasing the concentration gradient.

Increasing dissolved oxygen in plasma by oxygen therapy may also be used to offset the effects of hypoperfusion to some extent (stagnant hypoxia) and may well be important in certain situations (cardiogenic shock), although the effect is only marginal. Increased inspired oxygen will only marginally mitigate the effects of anaemic hypoxia but, because the $\mathrm{CaO}_{2}$ in patients with anaemia is less than that in patients with normal haemoglobin, the effect of additional oxygen carried in solution may become more important in these situations.

\subsection{Strategies for improving oxygenation and delivery}

Tissue oxygenation is dependent upon optimal or adequate oxygen delivery to the tissue $\left(\mathrm{DO}_{2}\right)$. This physiological process is composed of various components that independently and interdependently influence and determine $\mathrm{DO}_{2}$ and therefore 


\section{Example 2}

For a patient with sepsis and anaemia with a haemoglobin level of $50 \mathrm{~g} / \mathrm{l}(5 \mathrm{~g} / \mathrm{dl})$ and an oxygen saturation of $90 \%$, increasing oxygen saturation from $90 \%$ to $100 \%$ will increase the total haemoglobin oxygen content by about $10 \%$.

- Transfusing 2 units of packed red cells will increase the total haemoglobin oxygen content by about $40 \%$.

tissue oxygenation. These components can be considered sequentially.

\subsubsection{Optimising $\mathrm{PaO}_{2}$}

The physiology of oxygen therapy has already been discussed in the previous section. However, increasing $\mathrm{FIO}_{2}$ is only one component in increasing oxygen uptake in the lungs. Other key manoeuvres to ensure oxygen delivery to the alveolar capillary bed include:

- Maintaining a satisfactory airway.

- Ensuring adequate alveolar ventilation.

- Reversing any respiratory depressants such as narcotics.

- Invasive or non-invasive ventilation where necessary.

- Treating airflow obstruction by bronchodilation or sputum clearance techniques.

- Optimising transfer factor (diffusion capacity).

- Treatment of pulmonary oedema.

\subsubsection{Optimising oxygen carriage}

Oxygen is carried in blood mainly by haemoglobin with only a very small amount of oxygen dissolved in the blood itself. Adequate haemoglobin is therefore essential for optimal oxygen content $\left(\mathrm{CaO}_{2}\right)$ of blood. The ideal haemoglobin level for optimal $\mathrm{CaO}_{2}$ and therefore for optimal $\mathrm{DO}_{2}$ has long been a subject of debate. Previous practices have favoured haemoglobin levels close to $100 \mathrm{~g} / \mathrm{l}(10 \mathrm{~g} / \mathrm{dl})$, providing adequate $\mathrm{CaO}_{2}$ as well as reducing viscosity of blood for better perfusion in critically ill patients. However, studies by Canadian researchers in the late 1990s have shown that haemoglobin levels of $70 \mathrm{~g} / 1$ (7 g/dl) were as safe as higher levels and may produce fewer complications in the critically ill. ${ }^{55}$ However, this study was conducted using non-leucocyte depleted blood and it is possible that some of the infective complications in the group who were given more transfusions might have been avoided by the use of leucocyte-depleted blood. The optimal transfusion target for critically ill patients therefore remains the subject of ongoing discussion among experts in critical care medicine. Although the issue of optimal haemoglobin in patients with unstable or symptomatic coronary artery disease is not settled, haemoglobin levels of $100 \mathrm{~g} / \mathrm{l}(10 \mathrm{~g} / \mathrm{dl})$ are recommended for adequate $\mathrm{DO}_{2}$ (see box).

\subsubsection{Optimising delivery}

Besides adequate $\mathrm{CaO}_{2}$ and $\mathrm{PaO}_{2}$, delivery of oxygen depends upon adequate flow of oxygenated blood. Cardiac output in turn depends upon adequate blood (circulating) volume, adequate venous return and adequate and optimal myocardial function. To avoid tissue hypoxia, attention must therefore be paid to the volume status of the patient and the adequacy of cardiac function, as well as initiating oxygen therapy. In severely shocked patients (eg, cardiogenic shock, septic shock), invasive monitoring and inotropic/vasopressor therapy will usually be indicated in appropriate higher dependency environments. It has been shown that deliberately increasing oxygen delivery in critically ill patients as well as high-risk surgical patients reduces organ failure, reduces length of ICU stay and, most importantly, improves mortality. ${ }^{56-59}$ Increased oxygen delivery partly involves oxygen therapy, but these studies did not show any benefit from aiming at supraphysiological oxygen delivery.

The following worked example illustrates how minor abnormalities in each of the parameters discussed above, when occurring together, can result in dramatic falls in oxygen delivery.

Oxygen delivery in health can be calculated as follows where $\mathrm{CO}$ is cardiac output and $\mathrm{Hb}$ is haemoglobin (normal values: $\left.\mathrm{CO}=5 \mathrm{l} / \mathrm{min} ; \mathrm{SaO}_{2}=94-98 \%, \mathrm{Hb}=15 \mathrm{~g} / \mathrm{l}\right)$ :

$$
\begin{aligned}
\mathrm{DO}_{2} & =\mathrm{CO} \times \mathrm{CaO}_{2} \\
\mathrm{Do}_{2} & =\mathrm{CO} \times\left\{\left[\mathrm{SaO}_{2} / 100 \times \mathrm{Hb} \times 1.3\right]+\left[\mathrm{PaO}_{2} \times 0.003 \mathrm{~mm} \mathrm{Hg} \times\right.\right. \\
10]\} &
\end{aligned}
$$

\section{Therefore:}

$$
\begin{aligned}
& \mathrm{Do}_{2}=5 \times\{[0.98 \times 150 \times 1.3]+[100 \times 0.003 \times 10]\} \\
& \mathrm{Do}_{2}=970 \mathrm{ml} / \mathrm{min} \text { or } \approx 1000 \mathrm{ml} / \mathrm{min}
\end{aligned}
$$

This is well above the normal oxygen consumption $\left(\mathrm{VO}_{2}\right)$ of about $250 \mathrm{ml} / \mathrm{min}$.

Now consider an anaemic patient with a haemoglobin level of $10 \mathrm{~g} / \mathrm{dl}$, cardiac output $3.5 \mathrm{l} / \mathrm{min}$ and $\mathrm{SaO}_{2}$ of $90 \%$; the oxygen delivery becomes approximately $410 \mathrm{ml} / \mathrm{min}$ :

$\mathrm{Do}_{2}=3.5 \times\{[0.9 \times 100 \times 1.3]+[60 \times 0.003 \times 10]\}$

Although this value is still above the $\dot{\mathrm{V}}_{2}$ at resting physiology, in practice the $\mathrm{VO}_{2}$ would most likely have risen owing to a number of factors such as increased work of breathing and increased catabolic state of sepsis. This example is not rare and occurs daily in clinical practice. It is therefore important not to consider oxygen therapy in isolation. As many patients may not have adequate haemoglobin, cardiac output or blood volume, they may suffer from tissue hypoxia when they become acutely ill. All such patients should have supplemental oxygen therapy until they are evaluated by a responsible healthcare professional.

\section{SECTION 6: HYPOXIA, HYPEROXIA, HYPERCAPNIA AND THE RATIONALE OF TARGETED OXYGEN THERAPY}

\subsection{Effects and risks of hypoxia and rationale for target oxygen saturation range}

As this guideline is addressing emergency oxygen therapy, this section will only focus on the effects and risks of acute hypoxia. Section 8 will discuss the emergency treatment of acute hypoxia in patients with long-term diseases associated with chronic hypoxia. The approximate relationship between $\mathrm{PaO}_{2}$ and $\mathrm{SaO}_{2}$

\begin{tabular}{|c|c|c|c|c|c|c|c|c|c|c|c|c|c|c|}
\hline $\mathrm{PaO}_{2}(\mathrm{kPa})$ & 4 & 5 & 6 & 7 & 8 & 9 & 10 & 11 & 12 & 13 & 14 & 15 & 16 & $\geqslant 17$ \\
\hline $\mathrm{PaO}_{2}(\mathrm{~mm} \mathrm{Hg})$ & 30 & 37.5 & 45 & 52.5 & 60 & 67.5 & 75 & 82.5 & 90 & 97.5 & 104 & 112.5 & 120 & $\geqslant 127.5$ \\
\hline $\mathrm{SaO}_{2}(\%)$ & 57.4 & 71.4 & 80.7 & 86.8 & 90.7 & 93.2 & 94.9 & 96.2 & 97.0 & 97.8 & 98.2 & 98.6 & 98.8 & $\geqslant 99.0$ \\
\hline
\end{tabular}
is shown in table 8 and fig 3 (oxygen dissociation curve).

The effects and risks of hypoxia are summarised in table 9. Severe hypoxia may lead to brain damage and death. In general,

Table 8 Approximate relationship between arterial blood saturation $\left(\mathrm{SaO}_{2}\right)$ and arterial oxygen tension $\left(\mathrm{PaO}_{2}\right)^{60}$ 
Table 9 Physiological effects of acute hypoxia and hyperoxia

\begin{tabular}{|c|c|c|c|c|}
\hline & \multicolumn{2}{|l|}{ Hypoxia } & \multicolumn{2}{|l|}{ Hyperoxia } \\
\hline & Effects & Risks & Effects & Risks \\
\hline \multirow[t]{2}{*}{ Respiratory system } & $\begin{array}{l}\text { Increased ventilation } \\
\text { Pulmonary }\end{array}$ & \multirow[t]{2}{*}{ Pulmonary hypertension } & \multirow[t]{8}{*}{$\begin{array}{l}\text { Decreased ventilation } \\
\text { (minimal) }\end{array}$} & $\begin{array}{l}\text { Worsened ventilation/ } \\
\text { perfusion matching }\end{array}$ \\
\hline & vasoconstriction & & & \multirow{4}{*}{$\begin{array}{l}\text { Absorption atelectasis } \\
\text { Myocardial ischaemia (in } \\
\text { context of decreased } \\
\text { haematocrit) } \\
\text { Reduced cardiac output }\end{array}$} \\
\hline \multirow{6}{*}{$\begin{array}{l}\text { Cardiovascular } \\
\text { system }\end{array}$} & Coronary vasodilation & \multirow{2}{*}{$\begin{array}{l}\text { Myocardial ischaemia/ } \\
\text { infarction }\end{array}$} & & \\
\hline & Decreased systemic & & & \\
\hline & $\begin{array}{l}\text { vascular resistance } \\
\text { (transient) }\end{array}$ & \multirow{2}{*}{$\begin{array}{l}\text { Ischaemia/infarction of } \\
\text { other critically perfused } \\
\text { organs }\end{array}$} & & \\
\hline & Increased cardiac output & & & Reduced coronary blood flow \\
\hline & Tachycardia & Hypotension & & Increased blood pressure \\
\hline & & Arrhythmias & & $\begin{array}{l}\text { Increased peripheral } \\
\text { resistance }\end{array}$ \\
\hline \multirow[t]{2}{*}{ Metabolic system } & Increased 2,3-DPG & \multirow[t]{2}{*}{ Lactic acidosis } & Decreased 2,3-DPG & \multirow{5}{*}{$\begin{array}{l}\text { Increased reactive oxygen } \\
\text { species }\end{array}$} \\
\hline & $\begin{array}{l}\text { Increased } \mathrm{CO}_{2} \text { carriage } \\
\text { (Haldane effect) }\end{array}$ & & $\begin{array}{l}\text { Decreased } \mathrm{CO}_{2} \\
\text { carriage (Haldane } \\
\text { effect) }\end{array}$ & \\
\hline \multirow[t]{3}{*}{ Neurological system } & \multirow{3}{*}{$\begin{array}{l}\text { Increased cerebral blood } \\
\text { flow due to vasodilation }\end{array}$} & Confusion & \multirow{3}{*}{$\begin{array}{l}\text { Decreased cerebral } \\
\text { blood flow }\end{array}$} & \\
\hline & & Delirium & & \\
\hline & & Coma & & \\
\hline \multirow[t]{2}{*}{ Renal system } & $\begin{array}{l}\text { Renin-angiotensin axis } \\
\text { activation }\end{array}$ & \multirow[t]{2}{*}{ Acute tubular necrosis } & & \multirow[t]{2}{*}{ Reduced renal blood flow } \\
\hline & $\begin{array}{l}\text { Increased erythropoietin } \\
\text { production }\end{array}$ & & & \\
\hline
\end{tabular}

many of the physiological effects of hypoxia are mediated by low $\mathrm{PaO}_{2}$, irrespective of oxygen content. For example, even when the total blood oxygen content is normal in the presence of polycythaemia, hypoxaemia will still exert a physiological effect such as stimulation of ventilation. The risks of hypoxia, however, are usually mediated by low tissue $\mathrm{PO}_{2}$ which may occur as a consequence of a low $\mathrm{PaO}_{2}$ and other mechanisms such as severe anaemia and low cardiac output states.

These problems can be illustrated in the pathophysiology of myocardial ischaemia which will develop when there is an imbalance between myocardial $\mathrm{Do}_{2}$ and oxygen consumption $\left(\mathrm{VO}_{2}\right) . \mathrm{DO}_{2}$ is not only dependent on $\mathrm{PaO}_{2}$, but also coronary flow and haematocrit. $\mathrm{VO}_{2}$ will also depend on the stroke work of the heart. Defining a lower limit of $\mathrm{PaO}_{2}$ which is considered safe is therefore impossible given the other variables.

Hypoxaemia refers to an abnormally low oxygen tension in the blood (see section 3.1). However, it is not possible to define a single level of hypoxaemia that is dangerous to all patients. Some patients with chronic lung disease may be accustomed to living with $\mathrm{SaO}_{2}$ as low as $80 \%\left(\mathrm{PaO}_{2}\right.$ about $6 \mathrm{kPa}$ or $\left.45 \mathrm{~mm} \mathrm{Hg}\right)$ while other patients with acute organ failure may be harmed by shortterm exposure to $\mathrm{SaO}_{2}<90 \%\left(\mathrm{PaO}_{2}<8 \mathrm{kPa}\right.$ or $\left.60 \mathrm{~mm} \mathrm{Hg}\right)$.

It has been shown that medical patients with sustained desaturation $<90 \%$ have impaired medium-term survival compared with medical patients with saturations which stay $>90 \%{ }^{21}$ However, much of this survival disadvantage may be due to the underlying disease which has caused the low oxygen level (eg, severe COPD or pneumonia) and the degree of hypoxaemia may be a marker of disease severity, therefore the contribution of modest hypoxaemia to mortality rates is not known.

Mental functioning becomes impaired if the $\mathrm{PaO}_{2}$ falls rapidly to $<6 \mathrm{kPa}\left(45 \mathrm{~mm} \mathrm{Hg}, \mathrm{SaO}_{2}<80 \%\right.$ ) and consciousness is lost at $<4 \mathrm{kPa}\left(30 \mathrm{~mm} \mathrm{Hg}, \mathrm{SaO}_{2}<56 \%\right)$ in normal subjects. ${ }^{61-64}$ Young subjects tolerate acute hypoxaemia for longer than older subjects in terms of "time of useful consciousness". ${ }^{64}$ Safe levels of hypoxaemia in COPD have been discussed in detail in a review by Murphy and colleagues. ${ }^{10}$ Many patients with COPD have a $\mathrm{PaO}_{2}$ of $<5 \mathrm{kPa}$ (37.5 $\mathrm{mm} \mathrm{Hg}$ ) corresponding to a $\mathrm{SaO}_{2}$ of $<70 \%$ during an acute exacerbation. ${ }^{65}$ Furthermore, sudden hypoxaemia is more dangerous than hypoxaemia of gradual onset both in health and in disease. For example, millions of people live at altitudes above $3000 \mathrm{~m}$ despite an average $\mathrm{PaO}_{2}$ of about $7.3 \mathrm{kPa}(55 \mathrm{~mm} \mathrm{Hg}$, saturation about 88\%) and acclimatised climbers on Mount Everest can tolerate short-term exposure to an oxygen saturation of $70 \%$ or less with an estimated $\mathrm{PaO}_{2}$ of about $3.7 \mathrm{kPa}$ $(28 \mathrm{~mm} \mathrm{Hg}){ }^{18-20} 27$ Campbell summarised this issue eloquently in 1967 when he said "Better a year at a $\mathrm{PaO}_{2}$ of $50 \mathrm{~mm} \mathrm{Hg}$ $(6.7 \mathrm{kPa})$ than an hour at a $\mathrm{PaO}_{2}$ of $20 \mathrm{~mm} \mathrm{Hg}(2.7 \mathrm{kPa})$ ").66 Hypoxic hepatitis has been reported in patients with respiratory failure associated with oxygen levels below $4.5 \mathrm{kPa}$ or $34 \mathrm{~mm} \mathrm{Hg},{ }^{67}$ whereas hypoxic hepatitis in patients with cardiac disease is mainly due to decreased hepatic blood flow (stagnant hypoxia) and occurs at higher blood oxygen levels. ${ }^{68}$

An in-flight study of patients with COPD with mean $\mathrm{SaO}_{2}$ of $96 \%$ at sea level showed a fall to $90 \%$ in-flight in a commercial airliner and a further fall to a mean $\mathrm{SaO}_{2}$ of $87 \%$ while walking in the aircraft aisles. These patients had no symptoms during these hypoxaemic episodes. ${ }^{69}$ A study of 84 healthy airline passengers found that the mean $\mathrm{SaO}_{2}$ fell from $97 \%$ at ground level to $93 \%$ (1SD 85-98\%) at cruising altitude. ${ }^{70}$ A study of healthy airline cabin crew has shown that the $\mathrm{SaO}_{2}$ of flight attendants falls to a mean nadir of $88.6 \%$ without causing breathlessness or any other symptoms. Individual nadirs of $\mathrm{SaO}_{2}$ ranged from $93 \%$ down to $80 \% .^{71}$ Without any randomised evidence, the guideline production team have suggested that the level of saturation which is tolerated by healthy people without any symptoms (about $85 \%$ saturation) should be regarded as the safe lower limit of hypoxaemia. However, other co-morbidities may need to be taken into account and expert opinion recommends that the $\mathrm{SaO}_{2}$ should be maintained above $90 \%$ for seriously ill patients. ${ }^{21-24}$ For this reason, the present guideline recommends a target $\mathrm{SaO}_{2}$ (and $\mathrm{SpO}_{2}$ ) above $94 \%$ for most hypoxaemic patients to ensure that the actual oxygen level remains above $90 \%$ for most of the time with a $4 \%$ margin of safety to allow for oximeter error and artefact such as a weak signal or dark coloured skin. The accuracy of and pitfalls of 
oximetry are addressed in section 7.1.2. Specific targets for oxygen therapy in other diseases will be considered theoretically in this section and practically in sections 8 and 9. Some patients-especially older people or those with chronic lung disease-may have an $\mathrm{SaO}_{2}$ below $94 \%$ when clinically stable and oxygen should not be given just to maintain the $\mathrm{SaO}_{2}$ above $94 \%$ if the patient is clinically stable.

In assessing an ill patient, the $\mathrm{SaO}_{2}$ level is only one of several physiological variables that should be monitored. Many patients with sudden acute illness such as postoperative pulmonary emboli will have a sudden alteration in physiological variables as assessed by "track and trigger" systems such as the modified Early Warning Scoring systems (mEWS). ${ }^{72}$ Such patients may have only a small fall in $\mathrm{SaO}_{2}$ owing to physiological compensation mechanisms such as increased ventilation. Healthcare professionals therefore need to be alert for falls in $\mathrm{SaO}_{2}$ even within the recommended target ranges.

Because of the wide normal range for $\mathrm{SaO}_{2}$ and the uncertainty concerning the possible physiological consequences of minor hypoxaemia, there was more debate among the guideline group about desirable target ranges than about any other aspect of the guideline. If the $\mathrm{SaO}_{2}$ should fall slightly below $94 \%$, the key issue is to identify and treat the cause of the fall (eg, pulmonary embolism) rather than just correcting the hypoxaemia which is not of itself dangerous at this level. However, there is a danger that healthcare workers might fail to respond appropriately to abnormal hypoxaemia. After much debate it was concluded that the guideline would recommend a target range of $94-98 \%$ for all adult patients. This reflects the approximate normal range of $\mathrm{SaO}_{2}$ in healthy adults as discussed in section 3.1. However, a sustained fall in $\mathrm{SaO}_{2}$ of $>3 \%$, even within the normal range, may be an indicator of acute illness and should require clinical assessment of a patient while a minority or patients (especially those aged $>70$ years) may have an $\mathrm{SaO}_{2}$ of $<94 \%$ even when clinically stable.

\subsubsection{Desirable oxygen saturation ranges in acute illness}

- Acute hypoxaemia is considered dangerous to healthy subjects below a $\mathrm{PaO}_{2}$ of about $6 \mathrm{kPa}(45 \mathrm{~mm} \mathrm{Hg})$ or an $\mathrm{SaO}_{2}$ of about $80 \%$ due to impaired mentation and risk of tissue hypoxia, but patients with acute illness or chronic organ disease or ischaemia are likely to be at risk at $\mathrm{PaO}_{2}$ $>6 \mathrm{kPa}$. [Evidence level III]

- Changes in physiological "track and trigger" systems such as mEWS may occur in acute illness with either no change or only a small change in $\mathrm{SaO}_{2}$ levels. [Evidence level III]

- Critical illness may present initially with only a small fall in $\mathrm{SaO}_{2}$ level because of compensating mechanisms. [Evidence level IV]

- The upper end of the recommended range in this guideline $(98 \%)$ is the upper limit of $\mathrm{SaO}_{2}$ in healthy adults. [Evidence level III]

- The lower end of the suggested target saturation range $(94 \%)$ is about the lower end of the normal range and ensures that the $\mathrm{SaO}_{2}$ remains above $90 \%$ most of the time. [Evidence level III]

\subsection{Potential benefits of hyperoxaemia and supplemental oxygen therapy in non-hypoxaemic patients}

Supplemental oxygen therapy is most commonly given to correct hypoxaemia as discussed throughout this guideline. However, there are some circumstances where supplemental oxygen may be given to non-hypoxaemic patients.
6.2.1 Benefits of hyperoxaemia in carbon monoxide poisoning and pneumothorax

Hyperoxaemia is useful in some clinical situations. The best example of this is carbon monoxide (CO) poisoning. $\mathrm{CO}$ combines with haemoglobin and has a higher affinity for the haemoglobin molecule giving rise to carboxyhaemoglobin $(\mathrm{COHb})$. The half-life of $\mathrm{COHb}$ is about $4-5 \mathrm{~h}$ when breathing air but is reduced to about $40 \mathrm{~min}$ when breathing $100 \%$ oxygen. Hyperoxaemia may also be used to accelerate the resolution of pneumothorax in patients who do not require a chest drain. ${ }^{74} 75$

\subsubsection{Other potential benefits of oxygen therapy in non-hypoxaemic} patients

- Most guidelines for cardiopulmonary resuscitation and the care of patients with critical illness recommend the use of $100 \%$ oxygen in the initial stages of resuscitation. Although these recommendations are not evidence-based, it is unlikely that controlled trials would ever be undertaken using different levels of oxygen therapy in these emergencies and it seems intuitive to maximise oxygen delivery for critically ill patients with circulatory collapse. However, randomised trials have been undertaken of resuscitation of neonates breathing room air or oxygen and the unexpected outcome of a Cochrane review was that the outcome was possibly better when room air was used. ${ }^{76}$ This surprising finding cannot be extrapolated to adult patients, but it does emphasise the need for clinical trials even in areas where one might intuitively believe that oxygen would be beneficial. Furthermore, there is theoretical evidence that patients who have survived the initial stages of resuscitation may be managed more safely with $30 \%$ oxygen compared with $100 \%$ oxygen. ${ }^{77}$

- It has been shown that early intervention to increase oxygen delivery to the tissues in critically ill patients as well as highrisk surgical patients reduces organ failure, reduces length of ICU stay and, most importantly, improves survival. ${ }^{56-59}$ Increased oxygen delivery in part involves oxygen therapy, but these studies did not show any benefit from aiming at supraphysiological oxygen delivery.

- Short-term postoperative oxygen therapy (for $2 \mathrm{~h}$ ) has been shown to reduce the risk of surgical wound infections in double blind trials of patients having bowel surgery but not in general surgery. ${ }^{79-81}$

- Oxygen therapy has been reported to improve anastomotic integrity in animal models ${ }^{82}$ and to have potential benefit in human anastomotic surgery. ${ }^{83}$

- Reported benefits of oxygen therapy in healing of established wounds and in treatment of wound sepsis are controversial. Hyperbaric oxygen reduced the risk of amputation in patients with chronic diabetic foot ulcers and may improve the chance of healing over 1 year, but the Cochrane reviewers had concerns about the size and quality of existing studies and recommended further trials. ${ }^{84}$ It is not known if conventional oxygen therapy has any effect on wound healing. ${ }^{85}$

- Relief from cluster headaches has been reported in about $60 \%$ of cases but this observation is based on very small studies from the 1980s. ${ }^{86}{ }^{87}$ Although this could be considered as a form of emergency oxygen therapy, these patients are not breathless.

- Through relief of breathlessness and work of breathing, oxygen therapy may decrease carbon dioxide production and consequently offset some of the potential increase in $\mathrm{PaO}_{2}$ that might otherwise occur due to the mechanisms 
described in section 6.3.1. However, there are no controlled trials supporting the use of oxygen for this indication.

- Oxygen therapy may reduce nausea and vomiting in postoperative patients and in ambulances. Although some reports have suggested that oxygen may have a specific antiemetic effect during ambulance transfers and in the postoperative state, subsequent studies reported no effect on motion sickness and no anti-emetic effect in postoperative patients. ${ }^{8-92}$

\subsection{Potential adverse effects and risks of supplemental oxygen therapy and hyperoxaemia}

These are summarised in table 9 and in the review by Downs ${ }^{93}$ and in other sources. ${ }^{94-97}$ The following paragraphs will summarise the physiology and pathophysiology of supplemental oxygen therapy and hyperoxaemia.

\subsubsection{Respiratory system}

The most significant effect of excess oxygen on the respiratory system is hypercapnic respiratory failure in a population of vulnerable patients. This does not occur in the absence of significant pulmonary disease or musculoskeletal disease affecting the thorax, and it occurs while the $\mathrm{PaO}_{2}$ is still within the normal range or slightly below normal. There are at least five mechanisms responsible for this:

- V/Q mismatch.

- Ventilatory drive.

- Haldane effect.

- Absorption atelectasis

- Higher density of oxygen compared with air.

\section{V/O mismatch}

During air breathing, poorly ventilated alveolar capillary units will be hypoxic and therefore poorly perfused due to hypoxic pulmonary vasoconstriction (HPV). If high concentration oxygen is administered, the $\mathrm{PAO}_{2}$ will rise, thus reversing the HPV and increasing blood flow to that unit. However, although the oxygen in the unit has increased, it remains poorly ventilated with a high $\mathrm{PACO}_{2}$ and therefore a high pulmonary venous $\mathrm{PCO}_{2}$. As more blood is now passing through these units, the $\mathrm{PaCO}_{2}$ will rise. Normally when there is no significant lung disease or thoracic musculoskeletal disease, the respiratory system is able to compensate for these changes by increasing overall ventilation thereby lowering $\mathrm{PaCO}_{2}$. However, where respiratory mechanics are such that increased ventilation is not possible, $\mathrm{PaCO}_{2}$ will rise. Several authors have reported that this mechanism is more important than reduction in ventilatory drive in producing hypercapnia when supplementary oxygen is administered, but this continues to be a controversial area of respiratory physiology. ${ }^{98-105}$

\section{Ventilatory drive}

Hypoxaemia drives an increase in ventilation so it follows that relief of hypoxaemia will cause a decrease in ventilation. The consequent rise in $\mathrm{PaCO}_{2}$ is inversely proportional to the decrease in ventilation such that a halving of alveolar ventilation will lead to a doubling in $\mathrm{PaCO}_{2}$, assuming constant carbon dioxide production. As shown in fig 5, any increase in $\mathrm{PaO}_{2}$ above $8 \mathrm{kPa}(60 \mathrm{~mm} \mathrm{Hg})$ will not significantly reduce ventilation and increases above $13 \mathrm{kPa}(100 \mathrm{~mm} \mathrm{Hg})$ will have no impact on ventilaton as the carotid sinus discharge is largely attenuated above $13 \mathrm{kPa}$. This mechanism is therefore only of importance in explaining increases in $\mathrm{PaCO}_{2}$ when $\mathrm{PaO}_{2}$ rises to
$13 \mathrm{kPa}$, but not increases thereafter. This phenomenon is also seen in normal individuals. Several clinical studies have suggested that "hypoxic drive" makes only a small contribution to the rise in $\mathrm{PaCO}_{2}$ that is seen clinically when high-dose oxygen is given to patients with COPD, but one recent study has supported this mechanism. ${ }^{98-105}$

\section{Haldane effect}

The third effect of increasing $\mathrm{FIO}_{2}$ is to decrease the carbon dioxide buffering capacity of haemoglobin through the Haldane effect. $^{43}$

\section{Absorption atelectasis}

The fourth effect, absorption atelectasis, is thought to occur as a result of absorption of oxygen from alveoli with high $\mathrm{PAO}_{2}$ beyond obstructed airways. This can happen at $\mathrm{FIO}_{2}$ as low as $30-50 \%$ and will result in a shunt (increased $\mathrm{V} / \mathrm{O}$ mismatch). ${ }^{93}$

\section{Higher density of oxygen compared with air}

Johnson and colleagues ${ }^{106}$ have shown a reduction in forced expiratory volume in $1 \mathrm{~s}\left(\mathrm{FEV}_{1}\right)$ in patients who were breathing pure oxygen compared with breathing air. They concluded that this effect was probably related to the slightly increased density and viscosity of oxygen relative to air. This would increase the work of breathing which could contribute to hypercapnia in an exhausted patient.

It has been stated for several decades that "hyperoxaemia causes hypercapnic respiratory failure by producing decreased respiratory drive in patients with intrinsic lung disease, such as COPD", and many-if not most-medical textbooks from the 1960 s to the present time refer to "loss of hypoxic drive" as the main cause of hypercapnia and acidosis when high-dose oxygen is given to patients with an acute exacerbation of COPD. This assertion is usually attributed to Campbell who championed the concept of controlled oxygen therapy in the 1960s. However, Campbell has been widely misquoted. What he actually said in 1967 was as follows: "It is usual to attribute the rise in PaCO2 in these patients to removal of the hypoxic drive to ventilation but I share the doubts of Pain and co-workers ${ }^{103}$ that this is the whole story; changes in the pulmonary circulation may also be important". ${ }^{66}$ Most but not all subsequent studies have shown that Campbell was correct in this assumption..$^{98-105}$ Another curious feature of hypercapnia in acute exacerbations of COPD is that it is not universal. ${ }^{66}$ Some patients with COPD are prone to repeated episodes of hypercapnic respiratory failure and others may not ever suffer from this complication. Even among COPD patients with chronic hypercapnia, not all will develop an increased carbon dioxide level (and acidosis) during acute exacerbations.

The theory of "loss of hypoxic drive" as the cause of hypercapnia is further confounded by the observation that $\mathrm{PaCO}_{2}$ continues to rise as $\mathrm{PaO}_{2}$ is increased above $13 \mathrm{kPa}$ (100 mm Hg), which has little impact on decreasing ventilation and most patients with respiratory acidosis during an exacerbation of COPD have $\mathrm{PaO}_{2}$ above $10 \mathrm{kPa}$, equivalent to saturation above about $93 \% .^{34}$ Therefore, while a small reduction in ventilation may be a contributing factor to the rise in carbon dioxide levels during oxygen therapy in COPD, the major factor is the worsening of $\mathrm{V} / \mathrm{O}$ matching.

Additional effects of increasing $\mathrm{FIO}_{2}$ will relate to atelectasis and perhaps worsening airflow obstruction due to increased viscosity. In diseases where there is little intrinsic lung disease but significant respiratory muscle weakness, loss of hypoxic 
respiratory drive will be a greater factor in the development of hypercapnia. However, HPV remains a significant regulator of $\mathrm{V} / \mathrm{O}$ matching even in non-diseased lung.

\subsubsection{Rebound hypoxaemia following sudden cessation of supplementary oxygen therapy}

Patients who have developed worsened hypercapnic respiratory failure following high concentration oxygen therapy face a further significant danger of rebound hypoxaemia if oxygen is suddenly withdrawn in an attempt to correct the effects of excess oxygen therapy. Rebound hypoxaemia can be explained using the alveolar gas equation and, given its importance, is best illustrated with a working example (box).

For the purposes of simplicity, this example makes several assumptions such as a constant respiratory exchange ratio and alveolar-arterial gradient between stages 1 and 3 . It also assumes that ventilation remains unchanged. Although when $\mathrm{PaO}_{2}$ falls to $3.4 \mathrm{kPa}$ upon removal of oxygen, ventilation will rise, by definition it will not be able to rise sufficiently to meet the need to clear the carbon dioxide stores for the same reason that hypercanic respiratory failure developed in the first instance.

Rebound hypoxaemia is a major risk and may be more dangerous than the hypercapnic respiratory failure itself. Consequently, this guideline will recommend that oxygen therapy be stepped down gradually through sequential Venturi devices while monitoring saturation continuously.

- Sudden cessation of supplementary oxygen therapy can cause rebound hypoxaemia with a rapid fall in oxygen tension to below the tension that was present prior to the commencement of supplementary oxygen therapy. [Evidence level III]

\section{Example 3: Rebound hypoxaemia}

- Stage 1: Consider a patient with an exacerbation of COPD whose arterial blood gases are as follows: $\mathrm{PaO}_{2} 6.5 \mathrm{kPa} ; \mathrm{PaCO}_{2}$ $7.5 \mathrm{kPa}$ breathing room air. The $\mathrm{PAO}_{2}$ calculated from the alveolar gas equation (5.2.1) will be 11.6 assuming a respiratory exchange ratio of 0.8 , giving an alveolar-arterial gradient of $5.1 \mathrm{kPa}$.

- Stage 2: Given maximal oxygen through a reservoir bag mask, his blood gases become: $\mathrm{PaO}_{2} 32 \mathrm{kPa}$; $\mathrm{PaCO}_{2} 10 \mathrm{kPa}$. Because of the high solubility of carbon dioxide, the total body stores of carbon dioxide will have risen.

- Stage 3: If oxygen therapy is suddenly withdrawn, $\mathrm{PACO}_{2}$ and $\mathrm{PaCO}_{2}$ will remain high initially because of the high carbon dioxide stores and therefore $\mathrm{PAO}_{2}$ will fall further than it was initially before oxygen therapy to $8.5 \mathrm{kPa}$. Assuming the alveolar-arterial gradient for oxygen stays at $5.1 \mathrm{kPa}$ for room air breathing, then calculated $\mathrm{PaO}_{2}$ will become $3.4 \mathrm{kPa}$.

\subsubsection{Cardiovascular and cerebrovascular system}

The theoretical risks of hyperoxia have been summarised by Thomson and colleagues in an editorial which made a strong case for more trials. ${ }^{96}$ Hyperoxaemia causes coronary vasoconstriction and, if the haematocrit is sufficiently low, this may theoretically cause paradoxical myocardial hypoxia because of overall reduction in $\mathrm{DO}_{2}$.

One randomised double blind trial of oxygen in uncomplicated myocardial infarction found higher rates of sinus tachycardia and a significantly greater rise in myocardial enzyme in the oxygen group, suggesting a greater infarct size. ${ }^{107}$ There was a threefold increase in mortality in patients on oxygen therapy that did not reach statistical significance (3 deaths in 77 patients treated with air versus 9 deaths in 80 patients given oxygen at $6 \mathrm{l} / \mathrm{min}$ via simple face mask for $24 \mathrm{~h}$ ). This trial was published in 1976 and oxygen has been given routinely to millions of normoxaemic patients with myocardial infarction and chest pain for a further 30 years without any evidence to support the practice. Furthermore, a more recent trial showed increased mortality in patients with non-hypoxaemic strokes of mild to moderate severity in those randomised to treatment with oxygen. ${ }^{108}$ This creates an urgent need for large randomised trials of oxygen therapy for non-hypoxaemic patients with acute cardiac and cerebral ischaemia.

Thompson et $a^{96}$ have suggested that oxygen should be "prescribed, administered and monitored with care" in order "to achieve optimal tissue oxygenation", not maximal oxygenation. This view was proposed by other authors such as Bryan and Jenkinson ${ }^{109}$ in the 1980s, but standard medical practice has not taken note of this advice. Because there are no published data suggesting benefit from hyperoxaemia for most medical conditions and because of the theoretical risks, optimal management should aim for physiological oxygenation. Targets for oxygen therapy in specific circumstances, with evidence, are discussed in section 8 .

\subsubsection{Reactive oxygen species}

Aside from the potentially detrimental physiological effects of hyperoxaemia, the toxic effects mediated by reactive oxygen species (ROS) have potential risk. ${ }^{109}$ Excess ROS are generated in the presence of high tissue $\mathrm{PO}_{2}$ in the form of hydrogen peroxide and superoxide, causing oxidative stress and free radical damage. At physiological levels ROS act as signalling molecules, but at higher levels they are cytotoxic, notably being released by primed neutrophils as a host defence mechanism. It is thought that ROS are responsible for the development of bronchopulmonary dysplasia in ventilated hyperoxygenated premature infants ${ }^{110}$ and reperfusion injury post-myocardial infarction. ${ }^{111}$

\subsubsection{Delay in recognition of physiological deterioration}

It was previously believed that a high $\mathrm{FIO}_{2}$ is protective and gives patients a margin of safety. However, Downs and Beasley have argued that unstable patients may actually be placed at risk by the precautionary use of high-dose oxygen therapy. ${ }^{93} 97112$ During physiological deterioration, a patient given high-dose oxygen therapy would have a normal or high pulse oximeter reading masking a progressive decline in the $\mathrm{PaO}_{2} / \mathrm{FIO}_{2}$ ratio and therefore not alerting staff to impending deterioration requiring mechanical support. Furthermore, a patient who deteriorated physiologically while at a low $\mathrm{FiO}_{2}$ would be detected early by pulse oximetry and could have the $\mathrm{FIO}_{2}$ increased while being transferred to an intensive care unit whereas a patient who was already receiving a high $\mathrm{FIO}_{2}$ would desaturate more slowly but, when the oximeter eventually detected desaturation, there would be fewer treatment options because increasing the $\mathrm{FIO}_{2}$ further would have little effect. $^{93} 97112$

6.3.6 Lung injury in patients with acute paraquat poisoning, bleomycin lung injury and acid aspiration

Oxygen is known to be hazardous to patients with paraquat poisoning, ${ }^{113} 114$ and oxygen potentiates bleomycin lung injury and 
may potentiate lung injury from aspiration of acids. ${ }^{115-117}$ Further details concerning these conditions are given in section 8.13.5.

\subsubsection{Summary of risks of hyperoxia and supplemental oxygen therapy \\ Physiological risks}

(1) Worsened $\mathrm{V} / \mathrm{Q}$ mismatch.

(2) Absorption atelectasis.

(3) Coronary and cerebral vasoconstriction.

(4) Reduced cardiac output.

(5) Damage from oxygen free radicals.

(6) Increased systemic vascular resistance.

\section{Clinical risks}

(1) Worsening of hypercapnic respiratory failure.

(2) Delay in recognition of clinical deterioration.

(3) Worse outcomes in mild to moderate stroke.

(4) Specific risk in patients with previous bleomycin lung damage or with paraquat poisoning or acid aspiration.

(5) Unknown risk-benefit balance in acute coronary artery disease with normal oxygen saturation.

- Uncontrolled supplemental oxygen therapy can be harmful to patients who are at risk of hypercapnic respiratory failure, especially if the $\mathrm{PaO}_{2}$ is raised above $10 \mathrm{kPa}$. [Evidence level IIa]

- High-dose oxygen therapy to produce hyperoxaemia (above normal oxygen saturation) can cause absorption atelectasis, myocardial ischaemia and unfavourable outcomes in some patient groups (eg, patients with mild and moderate strokes). [Evidence levels Ib-III]

\subsection{Risks of hypercapnia (and respiratory acidosis)}

Hypercapnia and respiratory acidosis are inextricably linked and are best considered together. If hypercapnia develops slowly (over several days), a patient will have renal compensation (retention of bicarbonate) and acidosis will not occur in most such cases. However, acute elevation of the blood carbon dioxide level produces respiratory acidosis and symptoms of hypercapnia. Some of the consequences of an elevated carbon dioxide tension are a consequence of the resulting acidosis. Sometimes the effect of a raised carbon dioxide tension on a particular organ system is opposed by an opposite effect of acidosis.

Carbon dioxide is a vasodilator and patients with hypercapnia may appear flushed with dilated peripheral veins and a bounding pulse. Cranial vasodilation may cause headache. Carbon dioxide in high concentrations has hypnotic effects and patients with hypercapnia may progress from drowsiness to confusion to coma. ${ }^{10}{ }^{118-122} \mathrm{~A}$ link has been shown between severe respiratory acidosis in acute COPD and an increased risk of death or requirement for mechanical ventilation. ${ }^{34}$ However, the problem of respiratory acidosis is not confined to patients with COPD. Depressed respiration for any reason will give rise to hypercapnia. Examples are opiate overdoses, obesity with hypoventilation and neuromuscular disorders affecting the muscles of respiration.

\subsubsection{Effects of a raised blood carbon dioxide level Nervous system}

Carbon dioxide exerts its effect either directly or as a consequence of acidosis. Hypercapnia increases cerebral blood flow and thereby may influence the cerebrospinal fluid pressure.
It is the main factor influencing the intracellular $\mathrm{pH}$ which has an important effect on cellular metabolism. It exerts an inert gas narcotic effect similar to that of nitrous oxide. It influences the excitability of neurones particularly relevant in the reticular activating system. Carbon dioxide can induce narcosis when the $\mathrm{PaCO}_{2}$ rises above $12-16 \mathrm{kPa}(90-120 \mathrm{~mm} \mathrm{Hg}) .{ }^{118}$

\section{Pulmonary circulation}

An elevated $\mathrm{PACO}_{2}$ causes vasoconstriction in the pulmonary circulation although the effect is less marked than that of hypoxia. ${ }^{123}$ In healthy subjects an end expiratory $\mathrm{PCO}_{2}$ of $7 \mathrm{kPa}$ (52 $\mathrm{mm} \mathrm{Hg}$ ) increases pulmonary vascular resistance by $32 \%$ which, along with raised cardiac output, increases mean pulmonary artery pressure by $60 \% .{ }^{124}$ Changes in $\mathrm{pH}$ are thought to be the primary factor responsible for carbon dioxide-mediated changes in the pulmonary vasculature. ${ }^{125} 126$ Consequently, as with HPV, changes in $\mathrm{PACO}_{2}$ help to match perfusion to ventilation.

\section{Respiratory system}

As explained in section 6.2.1, a raised carbon dioxide level may worsen hypoxia and its effects because the concentration of carbon dioxide in the alveolar gas reduces that of oxygen if the concentration of nitrogen remains constant. Also an increase in $\mathrm{PaCO}_{2}$ shifts the oxygen dissociation curve to the right.

\section{Cardiovascular system}

In general, both hypercapnia and acidosis have direct depressant effects on cardiac myocytes and vascular smooth muscle cells. ${ }^{127}$ These effects are normally opposed by the increase in catecholamines caused by the raised $\mathrm{PaCO}_{2}$. The overall effect of carbon dioxide on the cardiovascular system is therefore unpredictable. In artificially ventilated children a rise in carbon dioxide increases cardiac output and reduces total peripheral resistance and blood pressure tends to rise. ${ }^{128}$ Although an increase in carbon dioxide depresses heart rate, tachycardia is more common because of the effects of catecholamine stimulation overriding the depressant effects on the heart. Arrhythmias have been reported but are seldom clinically significant in normal subjects. Carbon dioxide is a systemic vasodilator.

\section{Kidneys}

Renal blood flow and glomerular filtration rate are reduced in the presence of high levels of $\mathrm{PaCO}_{2}$. If severe, this can lead to anuria.

\section{Blood electrolyte levels}

The acidosis that accompanies hypercapnia may cause a rise in potassium if the acidosis is severe and sustained.

\section{Endocrine system}

Hypercapnia increases plasma levels of both adrenaline and noradrenaline.

\subsubsection{Clinical signs}

The clinical signs of hypercapnia are produced by the physiological changes described above and are described in detail in section 7.2.1.

\subsection{Risks of acidosis}

The major effect of acidosis is depression of the central nervous system with severe acidosis $\left(\mathrm{pH}<7.0\right.$ or $\left[\mathrm{H}^{+}\right]>100 \mathrm{nmol} / \mathrm{l}$ ) 
causing disorientation and later coma. However, as described above, the effects of $\mathrm{pH}$ are inextricably linked with both hypoxia and hypercapnia. As a consequence of opposing effects of acidosis, hypoxia and hypercapnia on different target organs in individual patients together with the fact that derangements of all three components may occur at the same time, it is very difficult to predict the effects of acidosis per se in an individual patient. Furthermore, tissue hypoxia will exacerbate acidosis. The consequences will depend upon the interplay of the three variables, complicated by the effects of co-morbid disease states. It is well known that, in patients with COPD, a $\mathrm{pH}$ of $<7.30$ or $\left[\mathrm{H}^{+}\right]>50 \mathrm{nmol} / \mathrm{l}$ during an acute exacerbation is associated with a much worse prognosis. ${ }^{34}$

\subsection{Rationale of oxygen therapy}

Oxygen therapy is usually defined as the administration of oxygen at concentrations greater than those found in ambient air. It is usually undertaken to treat or prevent hypoxaemia, thereby preventing tissue hypoxia which may result in tissue injury or even cell death. In some circumstances such as carbon monoxide poisoning or cluster headache, oxygen therapy is used to achieve hyperoxia. There are no published trials supporting the use of oxygen to relieve breathlessness in non-hypoxaemic patients and there is evidence from randomised studies that oxygen does not relieve breathlessness compared with air in non-hyoxaemic patients with COPD who are breathless following exertion or in non-hypoxaemic patients with advanced cancer. ${ }^{129-132}$

At the tissue level, mitochondrial activity requires oxygen for aerobic ATP synthesis for cellular activity. $\mathrm{PaO}_{2}$ of dry air at sea level is $21.2 \mathrm{kPa}(159 \mathrm{~mm} \mathrm{Hg})$, but at the mitochondrion, $\mathrm{PO}_{2}$ is in the range of $0.5-3.0 \mathrm{kPa}(4-22 \mathrm{~mm} \mathrm{Hg})$ depending on tissue type and local metabolic activity. This gradient from atmosphere to mitochondrion is known as the oxygen cascade. There are many factors in this cascade that affect the final mitochondrial $\mathrm{PO}_{2}$ including alveolar gas exchange, oxygen transport in the blood and tissue perfusion. Under pathological conditions, any change in one step in this cascade may result in hypoxia at the mitochondrial level. Therefore, although not necessarily addressing the underlying cause of tissue hypoxia, increasing $\mathrm{FIO}_{2}$ with oxygen therapy is the simplest and quickest way of avoiding hypoxic tissue damage. Besides oxygen therapy, other steps are usually necessary to improve the delivery of oxygen to the tissue (see section 5.6).

\subsection{Target oxygen saturation in acute illness (see also sections 4.3 and 6.1 )}

Many disease states lead to a reduced oxygen level and it is standard practice for breathless patients to be treated with oxygen. However, there have been few controlled trials comparing different levels of inspired oxygen for patients with any of the common diseases that lead to hypoxaemia. It must also be remembered that oxygen therapy is only one of several strategies that may be used to increase tissue oxygen delivery for critically ill patients (section 5.6).

In many clinical situations oxygen therapy is applied without a specific end point in mind. It has been suggested by many studies that hyperoxia can have deleterious physiological and clinical effects (see section 6.3), albeit such effects are not widely reported in conditions other than COPD. However, potential for harm may well exist with hyperoxia and good medical practice should be followed as in all drug prescriptions. As the actual $\mathrm{PO}_{2}$ at the mitochondrial level is so variable and dependent on many variables other than $\mathrm{PaO}_{2}$, it is often difficult to set a minimum level of $\mathrm{PaO}_{2}$ below which definite cell damage will occur or above which the host is safe from the effects of hypoxic cell damage. In addition, it is not possible to monitor mitochondrial $\mathrm{PO}_{2}$ clinically and the only clinically available surrogate of mitochondrial hypoxia is lactate production. Although blood lactate levels are useful and indicate tissue hypoxia, it is a late marker and therefore is an insensitive tool. Thus, targets set for "ideal" blood gas levels are based on arbitrary goals. Owing to the natural decline in normal arterial oxygen levels with age, it has been suggested that the ideal target $\mathrm{PaO}_{2}$ can be determined by the following equation: ${ }^{133}$

Ideal $\mathrm{PaO}_{2}=13.3 \mathrm{kPa}-0.04 \times$ age (in years) or $100 \mathrm{~mm} \mathrm{Hg}$ $-0.3 \times$ age (in years)

In terms of oxygen saturation measured by the bedside, this would translate into an $\mathrm{SaO}_{2}$ of $94-98 \%$ in most situations. This strategy avoids tissue hypoxia in almost all patients and also avoids potential deleterious effects of hyperoxia. Thus, the standard practice should be to prescribe oxygen to a specific saturation $\left(\right.$ or $\mathrm{PaO}_{2}$ ) rather than in terms of $\mathrm{FIO}_{2}$.

Clearly, consideration will need to be given to patients who have oxygen-sensitive carbon dioxide retention and targets may well have to be set lower for these patients to strike a balance between achieving a desirable and safe $\mathrm{SaO}_{2} / \mathrm{PaO}_{2}$ and carbon dioxide retention. Specific disease states will be addressed in section 8.

Patients with moderate to severe hypoxaemia are usually breathless and have an increased respiratory rate. Apart from causing physical tiredness, this also increases work of breathing, therefore increasing both oxygen consumption and carbon dioxide production. In these circumstances, oxygen therapy may reduce the work of breathing and therefore reduce carbon dioxide production. Therefore, oxygen therapy should theoretically improve breathlessness in hypoxaemic patients. However, this effect has not been demonstrated in clinical trials involving patients who were breathless but not hypoxaemic. For example, a recent meta-analysis of all published blinded studies of short burst oxygen therapy for patients with COPD with breathlessness failed to confirm any clinical benefit despite the widespread belief of doctors and patients that oxygen relieves breathlessness in this condition. ${ }^{129}$ A systematic review of oxygen and airflow on the relief of dyspnoea at rest in patients with advanced disease of any cause found only lowgrade scientific evidence that oxygen and airflow improve dyspnoea in some patients with advanced disease at rest, and almost all of these subjects were hypoxaemic and already using oxygen therapy. ${ }^{134}$

\section{Recommendations}

1. This guideline recommends aiming to achieve a normal or near-normal oxygen saturation for all acutely ill patients apart from those at risk of hypercapnic respiratory failure. [Grade D]

2. The recommended target saturation range for acutely ill patients not at risk of hypercapnic respiratory failure is $\mathbf{9 4 - 9 8 \%}$. Some normal subjects, especially people aged $>70$ years, may have oxygen saturation measurements below $94 \%$ and do not require oxygen therapy when clinically stable. [Grade D]

3. Most non-hypoxaemic breathless patients do not benefit from oxygen therapy, but a sudden reduction of more than $3 \%$ in a patient's oxygen saturation within the target saturation range should prompt 
fuller assessment of the patient (and the oximeter signal) because this may be the first evidence of an acute illness. [Grade D]

4. For most patients with known COPD or other known risk factors for hypercapnic respiratory failure (eg, morbid obesity, chest wall deformities or neuromuscular disorders), a target saturation range of 88-92\% is suggested pending the availability of blood gas results. [Grade $\mathrm{C}$ ]

5. Some patients with COPD and other conditions are vulnerable to repeated episodes of hypercapnic respiratory failure. In these cases it is recommended that treatment should be based on the results of previous blood gas estimations during acute exacerbations because hypercapnic respiratory failure can occur even if the saturation is below $88 \%$. For patients with prior hypercapnic failure (requiring non-invasive ventilation or intermittent positive pressure ventilation) who do not have an alert card, it is recommended that treatment should be commenced using a $28 \%$ Venturi mask at $41 / \mathrm{min}$ in prehospital care or a $24 \%$ Venturi mask at $2-41 / \mathrm{min}$ in hospital settings with an initial target saturation of $88-92 \%$ pending urgent blood gas results. These patients should be treated as a high priority by emergency services and the oxygen dose should be reduced if the saturation exceeds $92 \%$. [Grade D]

\subsection{Effects of body positioning including restraint systems}

Appropriate positioning of a patient can maximise $\mathrm{V} / \mathrm{O}$ matching. In the healthy self-ventilating adult lung, $\mathrm{V} / \mathrm{O}$ matching improves from non-dependent to dependent areas. In lung disease there is a disruption of this pattern and, in these instances, appropriate positioning may be advantageous in optimising V/O matching, therefore improving gas exchange, oxygenation and carbon dioxide clearance. For these reasons, breathless patients usually prefer to sit upright or near upright provided they are able to do so.

The relationship between dependency and $\mathrm{V} / \mathrm{O}$ matching is maintained irrespective of the position of the subject. The physiology is then transferable into alternate side lying positions; for example, in left side lying the dependent lung (left) will have the better $\mathrm{V} / \mathrm{O}$ matching. This is important in the presence of asymmetrical lung pathology as the "good lung down" principle will maximise $\mathrm{V} / \mathrm{Q}$ matching.

Many unwell patients are nursed in the semi-recumbent and supine positions. These positions do not facilitate $\mathrm{V} / \mathrm{O}$ matching as in the upright and full side lying position due to the hindrance to expansion of the dependent lung by the diaphragm and chest wall. Even in healthy subjects the oxygen tension is $0.7 \mathrm{kPa}(5 \mathrm{~mm} \mathrm{Hg}$ ) lower in the supine position than in the upright position. ${ }^{12}$ Similarly, $10 \%$ of patients with right hemiparesis and concomitant chest disease were more hypoxaemic in the left lateral position. ${ }^{135}$ Where there is pathological lung disease and hence already significant $V / O$ mismatch, gas exchange may be further impaired. This is discussed in a review of the effects of position on oxygen saturation in acute stroke. ${ }^{136}$ Patients with acute stroke without respiratory co-morbidities may be permitted to adopt any body position that they find most comfortable, while those with respiratory compromise should be positioned as upright as possible, avoiding slouched or supine positions to optimise oxygenation. ${ }^{136}$

The semi-recumbent/supine position is commonly adopted in an ambulance. In addition, for safety, the patient is strapped into the stretcher using abdominal and chest restraints with their arms by their side. While there are a lack of specific data regarding this, physiological principles suggest that the use of such positioning and restraints would compromise both respiratory muscle function and gas exchange.

Finally, there are some rare patients with liver disease, cardiac shunts or lung fibrosis who have "platypnoea and orthodeoxia" which means that they are more hypoxic in the upright position. ${ }^{137}$ Other patients with scoliosis or with a paralysed hemidiaphragm may feel more comfortable with the "good lung up". These patients should be allowed to choose the position in which their breathing is most comfortable for them.

\section{Recommendation}

6. Because oxygenation is reduced in the supine position, fully conscious hypoxaemic patients should ideally be allowed to maintain the most upright posture possible (or the most comfortable posture for the patient) unless there are good reasons to immobilise the patient (eg, skeletal or spinal trauma). [Grade C]

\section{SECTION 7: CLINICAL AND LABORATORY ASSESSMENT OF HYPOXAEMIA AND HYPERCAPNIA}

\subsection{Assessment of hypoxaemia}

7.1.1 Clinical assessment of breathless patients and assessment of cyanosis

Clinicians examining a critically ill patient should remember the "ABC" of emergency medicine (airway, breathing, circulation). In the case of critically ill patients it may be necessary to secure the airway and resuscitate a patient before a detailed history can be obtained and before a full physical examination can be undertaken.

In assessing an ill patient the $\mathrm{SaO}_{2}$ level is only one of several physiological variables that should be monitored. Many patients with sudden acute illness such as postoperative pulmonary emboli will have a sudden alteration in physiological "track and trigger" variables as assessed by the modified mEWS system. ${ }^{72} 73138$ Such patients may have only a small fall in $\mathrm{SaO}_{2}$ due to physiological compensation mechanisms such as increased ventilation. Clinicians therefore need to be alert for falls in $\mathrm{SaO}_{2}$ even within the recommended target ranges.

\section{Recommendations}

7. Fully trained clinicans should assess all acutely ill patients by measuring pulse, blood pressure, respiratory rate and assessing circulating blood volume and anaemia. Expert assistance from specialists in intensive care or from other disciplines should be sought at an early stage if patients are thought to have major life-threatening illnesses and clinicians should be prepared to call for assistance when necessary including a call for a 999 ambulance in prehospital care or a call for the resuscitation team or ICU outreach team in hospital care. [Grade C-D]

8. Initial clinical assessment and subsequent monitoring of acutely unwell patients should include the use of a recognised physiological "track and trigger" systems such as the modified Early Warning Scoring system (mEWS), and a change in this score should require medical review even if there is no change in oxygen saturation. [Grade $\mathrm{C}$ ] 
Traditional clinical assessment of hypoxaemia involves clinical inspection of the skin and buccal mucous membranes to decide whether central cyanosis is present or absent. This is a difficult clinical skill, especially in poor lighting conditions. Clinical assessment of hypoxaemia is made even more unreliable by the presence of anaemia or polycythaemia. Some patients may have peripheral cyanosis due to poor peripheral circulation in the presence of normal $\mathrm{SaO}_{2}$. Several studies have shown that hypoxaemia is often not recognised by emergency medical service providers, especially if the patient does not complain of respiratory distress. ${ }^{139-141}$ A systematic review of the literature in 2005 reported that most hypoxaemic patients had at least one vital sign abnormality but skin colour was a poor indicator of hypoxaemia compared with pulse oximetry. ${ }^{31}$ For these reasons it is recommended that clinicians should not rely on visual assessments of "cyanosis" but should instead use pulse oximetry to obtain an accurate assessment of a patient's oxygen saturation.

The nature of a patient's presenting illness may make hypoxaemia a likely outcome, thus prompting a careful clinical search for evidence of cyanosis complemented by urgent pulse oximetry. This situation applies to many common acute illnesses such as heart failure, COPD exacerbation, pneumonia and pulmonary embolism. A study of 2276 patients with pneumonia showed that hypoxaemia was independently associated with six risk factors: age $>30$ years (odds ratio (OR) 3.2), COPD (OR 1.9), congestive heart failure (OR 1.5), respiratory rate $>24 / \mathrm{min}$ (OR 2.3 ), altered mental status (OR 1.6) and chest radiographic infiltrate involving $>1$ lobe (OR 2.2) ${ }^{28}$ Acutely ill patients with significant hypoxaemia are likely to have an increased pulse rate or respiratory rate and, for this reason, usually score at least 3 points on a mEWS. ${ }^{72} 738$ The respiratory rate is the single best predictor of severe illness. ${ }^{73}$ However, many patients with marked hypoxaemia may present with non-specific findings such as restlessness and confusion rather than breathlessness, and oxygen saturation has been shown to be an independent predictor of mortality in multivariate analysis of the outcome of emergency medical admissions. ${ }^{142}$ Furthermore, the work of Thrush et al ${ }^{143}$ on normal volunteers has shown that heart rate, blood pressure and respiratory rate are not reliable indicators of hypoxaemia down to saturation levels as low as $70 \%$. This would suggest that the changes in vital signs which are seen in most hypoxic patients are due to the underlying illness rather than hypoxaemia per se.

Hypoxaemia may be associated with increased or decreased ventilation. Although some hypoxaemic patients may have reduced levels of ventilation as a causative factor, the majority of hypoxaemic patients have increased minute ventilation in an attempt to increase the blood oxygen level. For example, a patient with an opiate overdose may have reduced ventilation causing hypoxaemia despite having structurally normal lungs, whereas a patient with pneumonia or major pulmonary embolism may have significant hypoxaemia due to ventilation-perfusion mismatch despite an increased level of ventilation. The first patient in this example may appear peaceful and non-distressed despite significant hypoventilation and hypoxaemia, while the second patient is likely to have increased ventilation and tachycardia. The clinician therefore needs to make separate assessments of a patient's oxygen saturation and level of ventilation.

Having completed the history and rapid assessment of the patient, more detailed physical examination may reveal signs of an illness such as major pleural effusion, major pneumothorax or unexpected heart failure that may prompt the clinician to anticipate the presence of hypoxaemia.

\section{Advice and recommendations for clinical assessment of patients with suspected hypoxaemia}

- The medical history should be taken when possible in an acutely breathless patient and may point to the diagnosis of a particular acute illness such as pneumonia or pulmonary embolism or an exacerbation of a chronic condition such as COPD, asthma or heart failure. [Evidence level IV]

- Physical examination may provide evidence of a specific diagnosis such as heart failure or a large pleural effusion, but it is common for the cause of breathlessness to remain undiagnosed until the results of tests such as chest radiographs are available. [Evidence level IV]

- Patients with severe hypoxaemia may present with a nonrespiratory manifestation such as confusion or agitation rather than breathlessness and cyanosis is a difficult physical sign to record confidently (especially in poor light or with an anaemic or plethoric patient). [Evidence level IV]

- Tachycardia and tachypnoea are commoner than a physical finding of cyanosis. [Evidence level III]

- Physiological "track and trigger" systems such as the Early Warning Scoring system (EWS or mEWS) are extremely valuable in identifying patients with life-threatening illness even if this is not immediately obvious from the patient's history. [Evidence level III]

\subsubsection{Value and limitations of pulse oximetry}

Clinical assessment of hypoxaemia has been revolutionised by the advent of pulse oximetry in much the same manner as the clinical assessment of blood pressure was transformed by the invention of the sphygmomanometer. However, it is common to see patients with acute respiratory illness who have had multiple measurements of their blood pressure but no record made of their oxygen saturation, peak expiratory flow or $\mathrm{FEV}_{1}$. In addition to the clinical consequences of underassessment, Howes et $a l^{144}$ and Macnab et $a l^{145}$ have reported that the availability of a pulse oximeter was highly cost-effective because the finding of normal oximetry (>94\%) in many patients allowed paramedics to use oxygen less frequently with a potential financial saving of up to $\$ 2324$ (approximately $£ 1200)$ per ambulance per annum.

Pulse oximetry measures haemoglobin oxygen saturation by detecting the absorption of light at two specific wavelengths that correspond to the absorption peaks of oxygenated and deoxygenated haemoglobin. Oximeters are less reliable at low saturation such as $80 \%$, but modern oximeters reflect the arterial oxygen saturation accurately at saturation above about $88 \% .{ }^{146-150}$ In almost all clinical circumstances covered by this guideline, patients with a saturation below $88 \%$ will be given intensive therapy to bring the saturation up to at least $90 \%$, so the inaccuracy of the instruments at very low saturation levels should not affect patient management.

In one study of 123 adult patients who had simultaneous measurements of pulse oximetry and arterial oxygen saturation measured in arterial blood gases, the $95 \%$ confidence interval for the median difference ranged from -0.6 to $+0.5 \%{ }^{148}$ It has been estimated that an oxygen saturation of $92 \%$ or above measured by pulse oximetry has a sensitivity of $100 \%$ and specificity of $86 \%$ for excluding hypoxaemia defined as an arterial oxygen saturation below $60 \mathrm{~mm} \mathrm{Hg}(8 \mathrm{kPa}) .{ }^{151}$ 
Oximetry may be less accurate in acutely ill patients on intensive care units, but there are no direct comparisons of the accuracy of pulse oximetry in critically ill patients compared with stable patients and healthy individuals. The study of Perkins and colleagues showed a mean $\mathrm{SpO}_{2}$ of $94.6 \%$ compared with a mean $\mathrm{SaO}_{2}$ of $95.9 \%$ from 1132 simultaneous oximeter and arterial blood gas measurements on an intensive care unit. ${ }^{152}$ Fortunately, this average difference of $1.3 \%$ was lower for pulse oximeter readings, thus allowing a margin of safety in most cases. This study also showed that fluctuations in oxygen saturation measured by oximetry tended to be greater than changes in arterial oxygen saturation measured with samples from an indwelling radial artery catheter.

Although oximetry is widely used, there are few clinical studies examining its utility. The Cochrane meta-analysis of the use of oximetry in perioperative monitoring of more than 20000 patients failed to show any reduction in complications or deaths where oximetry was used, although oxygen was given more often to patients who were monitored with pulse oximetry. ${ }^{153}$ The authors suggested that the correction of modest hypoxaemia probably does not have much effect on clinical outcomes.

Pulse oximetry gives no information concerning $\mathrm{pH}, \mathrm{PCO}_{2}$ or haemoglobin level. Blood gases and full blood count tests are therefore required as early as possible in all situations where these measurements may affect patient outcomes.

The accuracy of pulse oximetry is diminished in patients with poor peripheral perfusion which may occur chronically in conditions such as systemic sclerosis or acutely in patients with hypotension or hypovolaemia. However, it has been suggested that many types of oximeter may remain accurate at arterial pressures as low as $20 \mathrm{~mm} \mathrm{Hg}$ so long as the machine is able to obtain a reading despite the low pulse pressure. ${ }^{154}$ Most oximeters give an indication of the pulse signal strength. It is important to ensure that the oximeter has a good signal if technically possible, and the probe may need to be tried on different fingers or toes or on the earlobe to obtain the best available signal for the individual patient. There are some patients with poor perfusion for whom pulse oximetry measurements cannot be made. This includes patients with cold peripheries, severe hypotension and peripheral "shut down".

It must be remembered that oximetry gives a normal reading for oxygen saturation in most patients with anaemia because the oxygen saturation of the available haemoglobin is normal although the total amount of haemoglobin available for oxygen transport is reduced. These patients have normal oxygen saturation levels despite having "anaemic hypoxia" which may cause considerable reduction in the total oxygen content of the blood. It is often not recognised that a patient with an $\mathrm{SpO}_{2}$ of $98 \%$ but a haemoglobin of $7 \mathrm{~g} / \mathrm{dl}((7 \times 0.98 \times 1.34)=$ $9.2 \mathrm{ml} \mathrm{O}_{2} / \mathrm{dl}$ ) will have a greatly reduced blood oxygen content compared with a patient with a haemoglobin of $15 \mathrm{~g} / \mathrm{dl}$ and a saturation of $85 \%((15 \times 0.85 \times 1.34)=17 \mathrm{ml} \mathrm{O} / \mathrm{dl})($ each $\mathrm{g} / \mathrm{dl}$ haemoglobin when fully saturated carries $1.34 \mathrm{ml}$ oxygen).

The accuracy of oximetry is unreliable in the presence of carbon monoxide or methaemoglobin. Both of these substances have similar light absorption characteristics to oxyhaemoglobin so an apparently normal $\mathrm{SpO}_{2}$ in a patient with carbon monoxide poisoning or methaemoglobinaemia may be falsely reassuring. Carboxyhaemoglobin levels above $2 \%$ may cause falsely elevated $\mathrm{SpO}_{2}$ measurements. ${ }^{155}$ Many smokers will have carboxyhaemoglobin levels above this level shortly after smoking a cigarette, and the carboxyhaemoglobin level may be elevated to $15 \%$ in some smokers and up to $50 \%$ or more in acute carbon monoxide poisoning. It is not known if the reduced blood oxygen content in smokers who develop sudden illness within a few hours of smoking cigarettes has any effect on clinical outcomes, or if heavy smokers might benefit from a slightly higher target saturation range than non-smokers during the first few hours of a serious illness in an effort to maintain a similar blood oxygen content.

Skin pigmentation may also influence the accuracy of pulse oximetry readings (usually overestimation but sometimes underestimation). In particular, the accuracy of pulse oximetry is impaired in dark skinned subjects at saturation levels below 80-85\%. ${ }^{156-158}$ However, this should rarely be a problem in clinical practice if the saturation is maintained in the range suggested in the present guideline (94-98\% for most patients), although the work of Jubran and Tobin on ventilated subjects suggested that an oxygen saturation of $92 \%$ was useful in predicting a $\mathrm{PaO}_{2}$ above $60 \mathrm{~mm} \mathrm{Hg}(8 \mathrm{kPa})$ in ventilated white subjects but was less reliable in ventilated black subjects who sometimes had an $\mathrm{SpO}_{2}$ reading that was more than $4 \%$ above the directly measured $\mathrm{PaO}_{2} \cdot{ }^{23}$ In the case of sickle cell crisis, pulse oximetry may underestimate the level of oxygenation. ${ }^{159}$ In these circumstances, under-reading is safer than over-reading because no truly hypoxaemic patient would be denied oxygen therapy. However, another study found that pulse oximeters did not misdiagnose either hypoxaemia or normoxaemia during a sickle cell crisis provided a good wave signal was present. ${ }^{160}$

Oximeters can be affected by motion of the patient's hand, but this is less of a problem with modern oximeters than with older devices. ${ }^{161}$ Motion artefact is more of a problem if the patient also has reduced perfusion of the measuring site. ${ }^{162}$ A malpositioned oximeter sensor can cause artefact which can overestimate or underestimate the true oxygen saturation; this can be a particular problem during repositioning of ill patients. ${ }^{163}$

The site of oximetry is also important. Finger and earlobe measurements are more accurate than measurements from a probe applied to the toe, and finger probes may be more accurate than ear probes. ${ }^{164} 165$ Finally, clinical staff need to remember to remove nail varnish and false nails to avoid artefacts in oximetry measurements.

- Pulse oximeters are accurate to within 1-2\% of directly measured arterial oxygen saturation in most subjects but the error (usually overestimation but sometimes underestimation) is greater in dark skinned subjects, especially with very low saturation (below 80-85\%). [Evidence level IIa]

- The accuracy of oximeters in shock, sepsis and hypotension is largely unknown, but most errors are likely to result in falsely low readings which would result in additional oxygen being given. Most errors in oximetry are therefore not likely to place patients at risk, but it is important to ensure that the oximeter has a good signal and it is important to avoid artefact due to motion, nail varnish or other potential sources of error. [Evidence level IIa]

- Oximetry is a valuable clinical tool but subject to artefact and errors of interpretation. All clinical staff who use oximeters must therefore be trained in their use and made aware of the limitations of oximetry. [Evidence level IV]

- It is advised that oximetry measurements on sleeping patients should be recorded over several minutes to avoid the possibility of being misled by a normal transient nocturnal dip in oxygen saturation. [Evidence level III]

- Pulse oximetry can be misleadingly normal in smokers because of raised blood carboxyhaemoglobin levels which 
will cause a reduced blood oxygen content despite an apparently normal oxygen saturation and a normal oxygen tension. Patients who have smoked cigarettes in the previous $10 \mathrm{~h}$ may therefore be at increased risk from hypoxia. [Evidence level III]

\section{Recommendations}

9. Oxygen saturation, "the fifth vital sign", should be checked by trained staff using pulse oximetry in all breathless and acutely ill patients (supplemented by blood gases when necessary) and the inspired oxygen concentration should be recorded on the observation chart with the oximetry result. [Grade D]

10. The presence of a normal $\mathrm{SpO}_{2}$ does not always negate the need for blood gas measurements because pulse oximetry will be normal in a patient with normal oxygen tension but abnormal blood $\mathrm{pH}$ or $\mathrm{PCO}_{2}$ or with a low blood oxygen content due to anaemia. Blood gases and full blood count tests are therefore required as early as possible in all situations where these measurements may affect patient outcomes. [Grade D]

7.1.3 Arterial and arteriolised blood gases (indications for blood gas sampling are given in section 8.4 and recommendation 13)

Arterial blood gases are the "gold standard test" for assessing respiratory failure. However, recent studies have shown that arteriolised capillary gases from the earlobe (but not from the finger) can provide an assessment of $\mathrm{pH}$ and $\mathrm{PaCO}_{2}$ that is almost identical to that obtained from an arterial sample. ${ }^{166-170}$ In both acute and stable situations the earlobe specimen gives a $\mathrm{PO}_{2}$ measurement which is $0.5-1 \mathrm{kPa}(3.7-7.5 \mathrm{~mm} \mathrm{Hg})$ lower than the simultaneous arterial measurement with most of the divergence occurring at oxygen tensions above 8-10 $\mathrm{kPa}(60-$ $75 \mathrm{~mm} \mathrm{Hg}$. ${ }^{167} 170$ This means that most patients can be managed safely based on the $\mathrm{pH}$ and $\mathrm{PCO}_{2}$ levels measured from earlobe blood gases supplemented by oxygen saturation measured by a pulse oximeter. ${ }^{167}{ }^{170}$ In critically ill patients the initial specimen should be an arterial specimen to guarantee an accurate initial assessment, but capillary gases are especially valuable for monitoring progress of the blood gases as a patient stabilises.

Patients who have had simultaneous arterial and earlobe samples rated the earlobe puncture procedure as being considerably less painful than arterial puncture. ${ }^{171}$ However, the administration of local anaesthesia before arterial blood gas sampling produced a significant reduction in pain. ${ }^{172}$ There is a very small risk of arterial damage from arterial puncture, especially if the radial site is used. Most reports of hand ischaemia have involved indwelling radial artery cannulae, but the vessel could also be injured by needle puncture. ${ }^{173}$ The guideline therefore recommends that arteriolised earlobe specimens should be used more widely than at present as a safer and less painful alternative to arterial blood gas sampling and local anaesthetic should be used wherever possible for arterial blood gas sampling, but this is often not practical in medical emergencies and blood gas sampling should not be delayed in these circumstances. However, the accuracy of earlobe samples in shock or hypotension is not known and it is recommended that arterial blood gases should be used in all cases of shock or hypotension (systolic blood pressure $<90 \mathrm{~mm} \mathrm{Hg}$ ).

The technique of patient preparation, sample acquisition and sample processing for arteriolised capillary gases is complex and should only be undertaken by fully trained staff. Capillary gases are very vulnerable to errors in technique and they should only be implemented for emergency use in units where staff have been fully trained in their use and where the quality of the technique is monitored constantly.

\section{Use of arteriolised capillary blood gas measurements}

- Patients find earlobe specimens less painful than arterial puncture without local anaesthesia. [Level IIa]

- Arteriolised earlobe blood gases will provide accurate information about $\mathrm{PaCO}_{2}$ and $\mathrm{pH}$ but do not provide an accurate measurement of $\mathrm{PaO}_{2}$. [Level IIa]

- The earlobe specimen gives a $\mathrm{PO}_{2}$ measurement which is $0.5-1 \mathrm{kPa}$ (4-7.5 $\mathrm{mm} \mathrm{Hg}$ ) lower than the simultaneous arterial measurement with greater divergence at oxygen levels above $8-10 \mathrm{kPa}(60-75 \mathrm{~mm} \mathrm{Hg})$. [Level IIa]

- However, a combination of earlobe gases (to monitor $\mathrm{pH}$ and $\mathrm{PCO}_{2}$ ) and oximetry (to measure oxygen levels) will allow safe management of most patients, even in emergency settings. (The only published evidence is for patients with COPD but this finding is likely to be generalisable to most patients other than those with shock or poor peripheral circulation). [Level IV]

- The technique of patient preparation, sample acquisition and sample processing for arteriolised capillary gases is complex and should only be undertaken by fully trained staff. [Level IV]

\section{Recommendations}

11. For critically ill patients or those with shock or hypotension (systolic blood pressure $<90 \mathrm{~mm} \mathrm{Hg}$ ), the initial blood gas measurement should be obtained from an arterial specimen. However, for most patients who require blood gas sampling, either arterial blood gases or arteriolised earlobe blood gases may be used to obtain an accurate measure of $\mathrm{pH}$ and $\mathrm{PCO}_{2}$. However, the $\mathrm{PaO}_{2}$ is less accurate in earlobe blood gas samples (it underestimates the oxygen tension by $0.5-$ $1 \mathrm{kPa}$ ) so oximetry should be monitored carefully if earlobe blood gas specimens are used. [Grade B]

12. Local anaesthesia should be used for all arterial blood gas specimens except in emergencies or if the patient is unconscious or anaesthetised. [Grade B]

\subsubsection{Transcutaneous oxygen assessments}

Transcutaneous oxygen devices give different information from pulse oximetry. They are more sensitive to reduced perfusion and may be used to monitor tissue oxygenation in trauma patients but their use is beyond the scope of this guideline. ${ }^{174}$

\subsection{Assessment of hypercapnia and acidosis}

\subsubsection{Clinical assessment}

In patients with lung disease hypercapnia may be accompanied by visible respiratory distress, but this will be absent when hypercapnia is a consequence of a reduction in minute ventilation. Patients may have a flushed face, a full and bounding pulse and muscle twitching together with the characteristic flap of the outstretched hands. In severe cases consciousness may be depressed and convulsions may occur. Gross hypercapnia usually occurs with profound hypoxaemia and it is therefore difficult to disentangle the direct effect of hypercapnia per se. Coma will usually occur when the $\mathrm{PaCO}_{2}$ is in the range $12-16 \mathrm{kPa} \quad(90-120 \mathrm{~mm} \mathrm{Hg})$. 
Survival has been seen following a $\mathrm{PaCO}_{2}$ of $67 \mathrm{kPa}$ (500 mm Hg). ${ }^{175}$

The presence of hypercapnic respiratory failure can be anticipated in patients with severe exacerbations of COPD or other diseases such as severe neuromuscular disorders. Carbon dioxide is a vasodilator so patients with hypercapnia may develop headache. Carbon dioxide in high concentrations has hypnotic effects and patients with hypercapnia may progress from drowsiness with flapping tremor to confusion to coma. ${ }^{10}{ }^{118-122}$ A study of 127 episodes of acute respiratory acidosis showed that the best clinical predictors of respiratory acidosis were drow-

siness (OR 7.1), flushing (OR 4.1), the presence of known COPD (OR 3.3) and the presence of intercostal retraction (OR 2.9). ${ }^{176}$

Clinical signs of carbon dioxide retention include:

- Vasodilation producing flushing and warm peripheries with dilated blood vessels (including retinal veins).

- Bounding pulse.

- Drowsiness.

- Flapping tremor.

- Confusion.

- Coma.

7.2.2 Blood arterial and arteriolar gases (see section 7.1.3 for further details)

Arterial or arteriolised earlobe capillary blood gases will give an accurate estimation of $\mathrm{pH}$ and $\mathrm{PaCO}_{2} \cdot{ }^{166-168}$ The blood gases will need to be repeated in 30-60 min in patients with significant hypercapnia or acidosis to monitor the response to treatment. Patients with COPD who remain acidotic despite 30-60 min of standard treatment (including controlled low-dose oxygen therapy) are likely to need non-invasive ventilation. ${ }^{34}$

\subsubsection{Venous $\mathrm{PCO}_{2}$ sampling}

It has been suggested that the venous $\mathrm{PCO}_{2}$ level can be used to screen for hypercapnia in patients with acute respiratory disease. A study of 196 paired samples of arterial and venous blood from patients with acute respiratory disease showed that the $\mathrm{PCO}_{2}$ in the venous sample was an average of $0.77 \mathrm{kPa}$ (5.8 $\mathrm{mm} \mathrm{Hg}$ ) higher than the simultaneous arterial sample. ${ }^{177} \mathrm{~A}$ venous $\mathrm{PCO}_{2}$ below $6 \mathrm{kPa}(45 \mathrm{~mm} \mathrm{Hg}$ ) had 100\% sensitivity for eliminating the risk of hypercapnia (arterial $\mathrm{PCO}_{2}$ above $6 \mathrm{kPa}$ or $45 \mathrm{~mm} \mathrm{Hg}$ ), although the specificity was low at 57\% and there was more variation in other studies. ${ }^{178} 179$ For patients who are not at risk of metabolic acidosis, the presence of a satisfactory oxygen saturation measured by pulse oximetry and a venous $\mathrm{PCO}_{2}$ below $6 \mathrm{kPa}(45 \mathrm{~mm} \mathrm{Hg}$ ) can exclude the possiblility of significant arterial hypoxia or hypercapnia and may obviate the need for arterial blood gas measurements. However, venous $\mathrm{PCO}_{2}$ sampling is not widely used in clinical practice at present and the guideline committee have therefore made no recommendations on its use.

\subsubsection{Carbon dioxide monitors and non-invasive assessments of hypercapnia}

End-tidal carbon dioxide monitors are used primarily to confirm tracheal intubation during anaesthesia, intensive care and for any patients requiring endotracheal intubation. They are considered the "gold standard" by the Royal College of Anaesthetists. The absence of any detectable carbon dioxide output indicates a failed intubation. The management of intubated patients is outside the remit of this guideline.
End-tidal carbon dioxide monitors are also useful in the management of cardiac arrest and circulatory collapse. Very low levels of carbon dioxide excretion indicate very low (or absent) cardiac output and a low likelihood of survival. ${ }^{180-182}$ These devices are also useful in the care of intubated patients in the emergency department because, through visualising a typical "box wave form", they can confirm that the tube is in the airway even in the absence of carbon dioxide production during a cardiac arrest. The appearance of carbon dioxide may be the first sign of spontaneous circulation. ${ }^{183}$

End-tidal carbon dioxide measurements correlate poorly with arterial carbon dioxide levels in patients with COPD, but they may be useful in some research studies of hyperventilation syndromes. However, these devices are inaccurate in patients with airways disease and those with a high respiratory rate, so they should not be used in the management of patients with respiratory failure and they will not be discussed further in this guideline.

An exciting new possibility is the development of probes that can assess $\mathrm{PCO}_{2}$ as well as $\mathrm{SpO}_{2}$ from a single probe. Early studies indicate that such devices can be accurate in normal volunteers and there have been some encouraging preliminary studies in patients with respiratory disease. ${ }^{184} 185$ Transcutaneous carbon dioxide monitors are also being developed in association with transcutaneous oxygen monitors for use in patients with shock and critical illness. ${ }^{174}$

\section{SECTION 8: EMERGENCY OXYGEN USE IN HOSPITAL SETTINGS}

The hospital management of hypoxaemic patients is presented before the prehospital management because it represents the "ideal" management. Some readers may prefer to read section 9 (prehospital care) first because most patients receive prehospital care before hospital care, but the Guideline Development Group preferred to present the "ideal management" first.

\subsection{Assessment and immediate management of breathless patients on arrival in hospital}

Breathless patients may arrive in hospital directly (without prior assessment) or in ambulances where they will usually have been assessed by paramedics who may also have initiated emergency treatments including oxygen therapy. As discussed in section 7 of this guideline, assessment, triage and resuscitation of critically ill patients must be undertaken in parallel with the initiation of oxygen therapy and specific treatment must be given for the underlying medical condition. All critically ill patients and all patients at risk of hypercapnic respiratory failure should be triaged as very urgent and should have blood gases taken on arrival in hospital. Furthermore, all seriously ill patients should be assessed by senior clinicians as early as possible. In many cases this may involve liaison with intensive care specialists or with appropriate other specialists who can deal effectively with the patient's major medical or surgical problems.

- Readers are referred to section 7.1.1 and to disease-specific guidelines for advice concerning the immediate assessment and management of seriously ill patients.

- Readers are referred to section 10 for advice concerning choice of oxygen delivery devices and systems.

- Readers are referred to tables 1-4 and charts 1 and 2 (figs 1 and 2) for a summary of the key elements of oxygen therapy in common medical emergencies.

- Remember to ask for senior advice or specialist advice early in the care of profoundly ill patients. 


\subsection{Differences in management in hospital compared with a prehospital setting}

The immediate management of medical emergencies in hospital settings before blood gas results are available is similar in principle to management in the prehospital setting (section 9). The main priorities are to avoid harmful levels of hypoxaemia for all patients and to avoid harmful levels of hypercapnia for patients who are at risk of this complication. However, the amount of information available to the healthcare professionals increases rapidly in the hospital environment. The hospital management is presented before the prehospital management because it represents the "ideal" management. This may also be achievable in some prehospital settings such as a well equipped primary care centre. However, in many prehospital settings there will usually be less information available concerning a patient's history and physiology and less equipment available to assess and treat the patient.

Differences between hospital settings and prehospital settings include:

- Pulse oximetry is almost always available in hospital at present. These guidelines also recommend that pulse oximetry must be available in all locations where emergency oxygen is used (section 9.1).

- Blood gas results can be available within minutes of arrival in hospital.

- Additional diagnostic information may be available from history, clinical examination, test results and from the patient's hospital records.

- Additional equipment and resources are available (eg, ability to ventilate).

Because of the universal availability of oximetry in hospitals, it is rare for the hospital medical team to have to administer oxygen on the basis that a patient "might be hypoxaemic". However, initial "blind management" is sometimes necessary for patients with shock or with very poor peripheral circulation where a reliable pulse oximetry trace cannot be obtained. Arterial blood gases should be obtained as a matter of urgency in all such cases.

\subsection{Which patients need oxygen therapy?}

Supplementary oxygen therapy is required for all acutely hypoxaemic patients and for many other patients who are at risk of hypoxaemia, including patients with major trauma and shock. Most acutely breathless patients will require supplementary oxygen therapy, but there are some situations such as acute hyperventilation or diabetic ketoacidosis where an apparently breathless patient will not benefit from oxygen therapy. There are some other clinical situations such as carbon monoxide poisoning where a patient may benefit from oxygen therapy despite a lack of hypoxaemia or breathlessness because carbon monoxide binds more avidly than oxygen to the haemoglobin molecule.

\section{Recommendations}

- Oxygen saturation should be measured in all breathless and acutely ill patients (see recommendation 9).

- Oxygen therapy should be given to hypoxaemic patients (see table 1). Patients do not require oxygen therapy if their oxygen saturation is $94 \%$ or above (exceptions are carbon monoxide poisoning and pneumothorax; see sections 8.10.7 and 8.11.6). Patients on oxygen with $\mathrm{SpO}_{2}>98 \%$ may not require oxygen therapy or may require a lower dose (see recommendatons $1-3$ and table 1 ).
- All patients with shock, major trauma, sepsis or other critical illness should be managed initially with high concentration oxygen therapy from a reservoir mask. The dose can be adjusted subsequently once the results of blood gas estimations are known and/or the patient is stable (see table 1). [Grade D]

\subsection{Which patients require blood gas measurements?}

Blood gases should be measured as soon as possible in most emergency situations involving hypoxaemic patients ${ }^{186}$ and are essential in patients who may develop type 2 respiratory failure (carbon dioxide retention with risk of respiratory acidosis). Blood gases should also be checked (and the clinical situation should be reviewed) if the oxygen saturation should fall by more than three percentage points, even if the saturation remains within the target range. For example, a fall from $98 \%$ to $93 \%$ might be due to a significant event such as a pulmonary embolus. In this situation the saturation of $93 \%$ will not harm the patient but the patient will remain at serious risk until the pulmonary embolism is diagnosed and treated. If oximetry shows a patient to be hypoxaemic, the initiation of oxygen therapy should not be delayed while awaiting the results of blood gas measurements.

Blood gas measurements are not usually required for patients with no risk factors for hypercapnic respiratory failure and an oxygen saturation of $94 \%$ or above breathing air unless the patient requires blood gas estimation for other reasons such as suspected metabolic acidosis or diabetic ketoacidosis. The BTS asthma guideline recommends that arterial blood gas measurements need not be recorded in patients with acute asthma and an oxygen saturation above $92 \%$ and no life-threatening features. ${ }^{187}$ Arterial blood gas sampling can be technically difficult, especially for poorly perfused patients, and junior staff should ask for assistance from more senior staff in difficult cases.

Following initial clinical assessment and the availability of a pulse oximetry measurement, a decision can be made regarding the need for blood gas estimation within a few minutes of arrival in the hospital environment or if a previously stable patient develops breathlessness within a hospital environment. Oximetry will give no information concerning carbon dioxide or $\mathrm{pH}$ levels and a normal pulse oximetry level may provide false reassurance in patients on oxygen therapy who may have unexpected hypercapnia and acidosis. However, careful clinical assessment supplemented by the use of oximetry will allow the setting of an appropriate oxygen saturation target for different groups of patients until blood gas results are available.

If repeated blood gas estimations are required, the timing will depend on the indication. In general, the oxygen saturation (and $\mathrm{PaO}_{2}$ ) stabilises at a new higher level within a few minutes of increasing the dose of oxygen but the $\mathrm{PaCO}_{2}$ can take 30-60 min to equilibrate. The rise in blood oxygen level can be monitored with oximetry, so repeat blood gas tests are done mostly to assess critical illness (immediate sampling required) or to monitor $\mathrm{pH}$ and $\mathrm{PCO}_{2}$ levels (best done 30-60 min after increasing the dose of oxygen).

\section{Recommendation}

13. Blood gases should be checked in the following situations:

- All critically ill patients.

- Unexpected or inappropriate hypoxaemia $\left(\mathrm{Spo}_{2}\right.$ $<94 \%$ in patients breathing room air or oxygen) or any patient requiring oxygen to achieve the above 
target range. (Allowance should be made for transient dips in saturation to $90 \%$ or less in normal subjects during sleep). [Grade D]

- Deteriorating oxygen saturation or increasing breathlessness in a patient with previously stable hypoxaemia (eg severe COPD). [Grade D]

- Any previously stable patient who deteriorates and requires a significantly increased $\mathrm{FIO}_{2}$ to maintain a constant oxygen saturation. [Grade D]

- Any patient with risk factors for hypercapnic respiratory failure who develops acute breathlessness, deteriorating oxygen saturation, drowsiness or other symptoms of carbon dioxide retention. [Grade D]

- Breathless patients who are thought to be at risk of metabolic conditions such as diabetic ketoacidosis or metabolic acidosis due to renal failure. [Grade D]

- Acutely breathless or critically ill patients with poor peripheral circulation in whom a reliable oximetry signal cannot be obtained. [Grade D]

- Any other evidence from the patient's medical condition that would indicate that blood gas results would be useful in the patient's management (eg, an unexpected change in "track and trigger" systems such as a sudden rise of several units in the mEWS score or an unexpected fall in oxygen saturation of $3 \%$ or more, even if within the target range). [Grade D]

\subsection{Can arteriolised earlobe gases be used as a substitute for arterial blood gases?}

Readers are referred to section 7.1.3 for advice concerning when to use arterial blood gases and when to use arteriolised earlobe blood gases.

\subsection{Should oxygen be prescribed at a fixed "dose" or to achieve a target saturation?}

In the past, oxygen was prescribed at a fixed $\mathrm{FIO}_{2}$ or at a fixed flow rate via nasal cannulae or variable performance face masks. However, several audits have shown that many (or most) patients do not receive the prescribed dose of oxygen..$^{2-8}$ Furthermore, a patient's oxygen requirement may vary over time so the prescribed oxygen dose may be too high or too low even a short time after the prescription was written. For this reason it is recommended that oxygen should be prescribed to a target saturation range rather than prescribing a fixed dose of oxygen or fraction of inspired oxygen. This is analogous to an insulin "sliding scale" where the prescriber specifies a variable dose of insulin to achieve a target blood glucose range rather than prescribing a fixed dose of insulin. This will allow the appropriate healthcare professional-usually a doctor, nurse or physiotherapist-to adjust each patient's dose of oxygen to achieve the safest oxygen saturation range for each patient.

The prescriber may indicate a starting dose, device or flow rate, but there needs to be an agreed system for adjusting the oxygen dose upwards or downwards according to a patient's needs (see charts 1 and 2 (figs 1 and 2); sections 11.3.6 and 11.3.7 and charts 3 and 4 (figs 17 and 18)). As a patient improves, he or she is likely to require a lower $\mathrm{FIO}_{2}$ over a time period that will vary between patients. Most recovering patients will eventually require no supplemental oxygen. On the other hand, a deteriorating patient may need an increased dose of oxygen. This increase can be initiated by nursing staff or physiotherapists, but the requirement for an increased dose of oxygen is an indication for urgent clinical reassessment of the patient (and repeat blood gas measurements in most instances).

- It is recommended that oxygen should be prescribed to a target saturation range rather than prescribing a fixed dose of oxygen or fraction of inspired oxygen (see recommendations 1, 2, 4 and 5).

\subsection{What should be the target oxygen saturation range for patients receiving supplementary oxygen?}

8.7.1 0xygen saturation target range for most patients

As discussed in sections 4-6 of this guideline, there is no evidence of benefit from above normal oxygen saturation in most medical emergencies and there is evidence that excessive doses of oxygen can have adverse effects, even in some patients who are not at risk of hypercapnic respiratory failure. A target oxygen saturation range of $94-98 \%$ will achieve normal or near normal oxygen saturation for most patients who are not at risk of hypercapnic respiratory failure. Furthermore, the suggested lower limit of $94 \%$ allows a wide margin of error in the oximeter measurement, thus minimising the risk of any patient being allowed to desaturate below $90 \%$ due to inaccurate oximetry.

\subsubsection{Oxygen requirements for specific groups of patients}

- Patients with critical illness requiring high dose oxygen therapy are discussed in section 8.10 .

- Patients with medical emergencies which frequently cause breathlessness and hypoxaemia are discussed in section 8.11.

- Patients with COPD and other conditions that may predispose to type 2 respiratory failure are discussed in section 8.12.

- Medical emergencies for which oxygen is commonly given at present but is not actually indicated unless the patient is hypoxaemic are discussed in section 8.13.

\subsection{Importance of blood gas measurements in guiding oxygen therapy}

As soon as blood gas measurements are available, a patient's further treatment can be guided by the results of this test. For patients with a normal or low $\mathrm{PaCO}_{2}$ and no risk factors for hypercapnic respiratory failure, it is safe to aim at an oxygen saturation in the normal range (94-98\%). For patients with a raised $\mathrm{PaCO}_{2}$, a lower oxygen saturation is indicated (88-92\%), especially if the patient is acidotic. Non-invasive ventilation is recommended for patients with COPD who have hypercapnia and a $\mathrm{pH}<7.35\left(\left[\mathrm{H}^{+}\right]>45 \mathrm{nmol} / \mathrm{l}\right)$ despite $1 \mathrm{~h}$ of standard medical treatment including controlled oxygen therapy. ${ }^{25} 32$

\subsection{What should be the initial choice of oxygen delivery system in hospital settings?}

The technical and practical aspects of different oxygen delivery systems are discussed in section 10. For major trauma cases and for severely hypoxaemic patients without risk factors for hypercapnic respiratory failure, a non-rebreathing mask (reservoir mask) at $10-15 \mathrm{l} / \mathrm{min}$ is the suggested first choice. The delivery system and $\mathrm{FIO}_{2}$ may be adjusted later to a lower dose of oxygen as a patient improves or towards supported ventilation if the patient deteriorates. The majority of patients with modest hypoxaemia can be treated with nasal cannulae or a simple face mask at a flow rate which is adjusted to maintain the oxygen saturation in the target range for their specific clinical presentation. Chart 2 (fig 2) shows a suggested scheme that allows the oxygen level to be adjusted upwards or downwards in gradual increments depending on a patient's 
clinical progress (see also sections 11.3.6 and 11.3.7). Venturi masks are recommended for low-dose oxygen therapy because they deliver a more reliable oxygen concentration than nasal cannulae or variable flow masks. ${ }^{188}$ They can also be combined with a humidifier system when necessary (see section 10.6.3). The mask and/or flow should be rapidly changed if the initial choice does not achieve the target saturation.

\subsubsection{Devices used in emergency oxygen therapy in hospitals (see section 10 for further details)}

- High concentration oxygen from reservoir mask (10-15 l/ min) or bag-valve mask for critical illness or severe hypoxaemia or during resuscitation.

- Nasal cannulae (2-6 l/min) or simple face masks (5-10 l/ min) for medium-dose oxygen therapy.

- $24 \%$ Venturi mask at $2 \mathrm{l} / \mathrm{min}$ or $28 \%$ Venturi masks at $4 \mathrm{l} /$ min for patients at risk of hypercapnic respiratory failure (change to nasal cannulae at 1-2 1/min when the patient has stabilised).

- Tracheostomy masks for patients with prior tracheostomy (adjust flow to achieve desired saturation).

\subsection{Recommended oxygen therapy for major medical emergencies and critical illness (see also table 1)}

There are a number of major medical emergencies where patients are very likely to suffer from hypoxaemia. High-dose oxygen therapy from a reservoir mask at $10-15 \mathrm{l} / \mathrm{min}$ is recommended in the initial management of all such patients prior to stabilisation in a critical care area or high dependency unit. Following stabilisation, the dose of oxygen can be titrated downwards to maintain a target saturation of $94-98 \%$. It is recommended that patients with COPD or other risk factors for hypercapnia who develop a critical illness should be treated by emergency services in the same manner as other critically ill patients until urgent blood gas results become available because the primary issue is the critical illness. Critically ill patients with hypercapnia, hypoxaemia and acidosis will require immediate assessment by intensive care teams and will usually require intubation and mechanical ventilation.

\subsubsection{Cardiac arrest and other conditions requiring cardiopulmonary resuscitation (CPR)}

The 2005 guideline for Adult Advanced Life Support issued by Resuscitation Council UK recommends the use of non rebreathing reservoir masks (or $100 \%$ oxygen via a self-inflating bag mask system) to deliver the highest possible inspired oxygen level to patients requiring resuscitation. ${ }^{189}$ The present guideline endorses these proposals during the period of resuscitation. Subsequent management will depend on the underlying condition and the patient's degree of recovery. There is theoretical evidence that patients who have survived the initial stages of resuscitation may be managed more safely with $30 \%$ oxygen than with $100 \%$ oxygen. ${ }^{77} 78$

Some patients will require invasive ventilation following CPR, but others will recover rapidly and an oxygen saturation target of $94-98 \%$ is recommended during the convalescent period.

\section{Recommendation (see table 1)}

- Use high-dose oxygen from a reservoir mask at $15 \mathrm{l} / \mathrm{min}$ or bag-valve mask during resuscitation. [Grade D]
8.10.2 Critically ill patients including major trauma, shock and major sepsis

There is evidence that early intervention to normalise oxygen delivery to the tissues using volume expansion and vasoactive agents is beneficial in the management of critically ill patients with shock or sepsis, but there is no evidence of benefit from attempts to achieve supranormal oxygen delivery. ${ }^{56-58}$ 190-194 In fact, there is evidence that hyperoxia can cause a paradoxical decrease in whole body oxygen consumption in critically ill patients, ${ }^{195}$ and it has been demonstrated recently that hyperoxia can impair oxygen delivery in septic patients. ${ }^{196}$ Most such patients are at risk of multiorgan failure and therefore require intensive care assessment as a matter of urgency. Critical care consensus guidelines set $90 \%$ saturation as the minimum level below which oxygen saturation should not be allowed to fall, and the Surviving Sepsis Campaign guideline recommends a target arterial oxygen saturation of $88-95 \%$ for patients with sepsis. ${ }^{23} 24194$ However, these recommendations are based on directly measured arterial oxygen saturations in critical care settings with intensive levels of nursing and monitoring. The present guideline recommends a slightly higher target saturation range prior to the transfer of these seriously ill patients to critical care facilities.

For most critically ill or severely hypoxaemic patients, initial oxygen therapy should involve the use of a reservoir mask, aiming at an oxygen saturation of $94-98 \%$. If the patient has concomitant COPD or other risk factors for hypercapnic respiratory failure, the initial saturation target should also be 94-98\% pending the results of blood gas estimations and assessment by intensive care specialists. If critically ill COPD patients have hypercapnia and acidosis, the correction of hypoxaemia must be balanced against the risks of respiratory acidosis and ventilatory support using non-invasive or invasive ventilation should be considered.

It is also recognised that many patients with long bone fractures may develop hypoxaemia even in the absence of injury to the airway or chest (possibly due to opiate treatment and fat embolism) and they should be monitored with oximetry and given oxygen if necessary. ${ }^{30}{ }^{197-199}$ These patients, if not critically ill, should have a target oxygen saturation of $94-98 \%$ or $88-92 \%$ if they have co-existing COPD or other risk factors for hypercapnic respiratory failure.

\section{Recommendation (see table 1)}

- In critical illness, including major trauma and sepsis, initiate treatment with a reservoir mask at 10-15 $1 / \mathrm{min}$ and aim at a saturation range of $94-98 \%$. [Grade D]

\subsubsection{Near-drowning}

Survivors of near-drowning may have suffered inhalation of fresh or sea water into the lungs and may become hypoxaemic. Supplemental oxygen should be given to all patients with saturation below $94 \%$, aiming at a target saturation of $94-98 \%$.

\section{Recommendation (see table 1)}

- In cases of near-drowning, aim at an oxygen saturation of 94-98\%. [Grade D]

\subsubsection{Anaphylaxis}

Patients with anaphylaxis are likely to suffer from tissue hypoxia due to a combination of upper and/or lower airway obstruction together with hypotension. In addition to specific 
treatment of these problems, the Resuscitation Council UK recommends high concentration oxygen (10-15 l/min, presumably by reservoir mask if available) for patients with anaphylaxis. ${ }^{189}$ The present guideline would endorse this practice in the immediate management of anaphylaxis followed by a target saturation of $94-98 \%$ once the patient's condition has stabilised.

\section{Recommendation (see table 1)}

- In anaphylaxis, initiate treatment with a reservoir mask at $10-15 \mathrm{l} / \mathrm{min}$ and aim at a saturation range of $94-98 \%$. [Grade D]

\subsubsection{Major pulmonary haemorrhage or massive haemoptysis} Major pulmonary haemorrhage and massive haemoptysis can occur for a large number of reasons ranging from acute pulmonary vasculitis to erosion of a blood vessel by a lung tumour. In addition to specific treatment of the causative condition, most such patients require supplementary oxygen treatment. A target saturation range of $94-98 \%$ is recommended. Treatment should be initiated with high concentration oxygen via a reservoir mask and subsequently adjusted according to Chart 2 (fig 2) to maintain a saturation of 94 $98 \%$ pending the results of blood gas measurements.

\section{Recommendation (see table 1)}

- In pulmonary haemorrhage, aim at an oxygen saturation of 94-98\%. [Grade D]

\subsubsection{Major head injury}

Patients with major head injury are at risk of hypoxaemia and hypercapnia. They require urgent assessment and maintenance of airway patency, either through positioning, simple adjuncts or early intubation and ventilation to avoid further brain injury due to brain oedema which may be aggravated by hypercapnia. These patients should be referred immediately to appropriately trained specialists, even if this requires an interhospital transfer. Initial treatment should include high concentration oxygen via a reservoir mask pending availability of satisfactory blood gas measurements or until the airway is secured by intubation. Although hypoxaemia is common in patients with head injury, the relative contribution of hypoxaemia to outcome is not yet established. ${ }^{29}{ }^{200-204}$ All authors agree that hypoxaemia should be corrected, but a recent review of the literature concluded that there is no evidence of clinical benefit from hyperoxia in braininjured patients and a subsequent clinical study showed that normobaric hyperoxia did not improve brain metabolism in five patients with acute severe brain injury. ${ }^{205} 206$ There are no UK guidelines for oxygen therapy in the immediate phase after head injury, but US guidelines recommend maintaining an oxygen saturation above $90 \%$ for patients with acute brain injury. ${ }^{22}$ The present guideline advises giving supplementary oxygen if required to maintain an oxygen saturation in the range of 94$98 \%$.

\section{Recommendation (see table 1)}

- In cases of major head injury, aim at an oxygen saturation of 94-98\%. Initial treatment should involve high concentration oxygen from a reservoir mask at $10-151 /$ min pending availability of satisfactory blood gas measurements or until the airway is secured by intubation. [Grade D]

\subsubsection{Carbon monoxide poisoning}

Patients with carbon monoxide poisoning have a normal level of $\mathrm{PaO}_{2}$ but a greatly reduced level of oxygen bound to haemoglobin because this has been displaced by carbon monoxide. ${ }^{207}$ Pulse oximetry cannot screen for carbon monoxide exposure as it does not differentiate carboxyhaemoglobin from oxyhaemoglobin and blood gas measurements will show a normal $\mathrm{PaO}_{2}$ in these patients. The blood carboxyhaemoglobin level must be measured to assess the degree of carbon monoxide poisoning. The half-life of carboxyhaemoglobin in a patient breathing room air is approximately $300 \mathrm{~min}$; this decreases to 90 min with high concentration oxygen via a reservoir mask. The most important treatment for a patient with carbon monoxide poisoning is therefore to give high-dose oxygen via a reservoir mask. Comatose patients or those with severe mental impairment should be intubated and ventilated with $100 \%$ oxygen. The role of hyperbaric oxygen remains controversial. A 2005 Cochrane review concluded that existing randomised trials did not establish whether the administration of hyperbaric oxygen to patients with carbon monoxide poisoning reduced the incidence of adverse neurological outcomes. ${ }^{208}$ However, a randomised trial published in 2007 has suggested that patients with loss of consciousness or high carboxyhaemoglobin levels may have less cognitive sequelae if given hyperbaric oxygen. ${ }^{209}$

\section{Recommendation (see table 1)}

- In cases of carbon monoxide poisoning, an apparently "normal" oximetry reading may be produced by carboxyhaemoglobin, so aim at an oxygen saturation of $100 \%$ and use a reservoir mask at $15 \mathrm{l} / \mathrm{min}$ irrespective of the oximeter reading and $\mathrm{PaO}_{2}$. [Grade $\mathbf{C}$ ]

\subsection{Serious illnesses requiring moderate levels of supplemental oxygen if the patient is hypoxaemic (see also table 2)}

Patients who present with acute medical emergencies who are not critically ill or grossly hypoxic can be treated with medium-dose oxygen therapy from nasal cannulae or a simple face mask with a target saturation range of $94-98 \%$. Some of these patients (eg, patients with pneumonia) may subsequently deteriorate, requiring high concentration oxygen from a reservoir mask or requiring respiratory support such as invasive ventilation. Others may turn out to have an additional diagnosis of COPD or neuromuscular disease with a risk of hypercapnic respiratory failure and they should be managed with a Venturi mask or 2 litres of oxygen via nasal cannulae, aiming at a target saturation of $88-92 \%$. There are no published trials supporting the use of oxygen to relieve breathlessness in non-hypoxaemic patients, and there is evidence from randomised studies that oxygen does not relieve breathlessness compared with air in non-hypoxaemic patients with COPD who are breathless following exertion. ${ }^{129}$

8.11.1 Patients with acute onset of hypoxaemia of unknown cause with no pre-existing respiratory disorders or risk factors

It is common for breathless and hypoxaemic patients to have no firm diagnosis at the time of presentation. For most acutely hypoxaemic patients whose medical problem is not yet diagnosed, an oxygen saturation range of $94-98 \%$ will avoid the potential hazards associated with hypoxaemia or hyperoxia (see sections 4-6 and table 1). Aiming for an oxygen saturation in the normal range (rather than an abnormally high oxygen 
level) will also have the effect of allowing the lowest effective $\mathrm{FIO}_{2}$ to be used, thus avoiding risks such as absorption atelectasis and $\mathrm{V} / \mathrm{O}$ mismatch that may be associated with the use of very high fractions of inspired oxygen (see sections 5 and 6). The priority for such patients is to make a specific diagnosis as early as possible and to institute specific treatment for the underlying condition. Early blood gas measurement is mandatory in the management of patients with sudden unexplained hypoxaemia.

\section{Recommendations (see table 2)}

- For acutely breathless patients not at risk of hypercapnic respiratory failure who have saturations below $85 \%$, treatment should be commenced with a reservoir mask at $10-15 \mathrm{l} / \mathrm{min}$ in the first instance. The oxygen dose can be adjusted downwards (using nasal cannulae or a simple face mask) to maintain a target saturation of $94-98 \%$ once the patient has stabilised. [Grade D]

- In all other cases without risk factors for hypercapnic respiratory failure, treatment should be commenced with nasal cannulae (or a simple face mask if cannulae are not tolerated or not effective) with the flow rate adjusted to achieve a saturation of 94-98\%. [Grade D]

- If medium-dose therapy with nasal cannulae or a simple face mask does not achieve the desired saturation, change to a reservoir mask and seek senior or specialist advice. [Grade D]

\subsubsection{Acute asthma}

The BTS/SIGN guideline for the management of acute asthma recommends that the oxygen saturation should be maintained above $92 \% .{ }^{187}$ The present guideline suggests a target saturation of $94-98 \%$ for most disease conditions, including asthma. The lower limit of $94 \%$ in this guideline is recommended in order to maintain consistency throughout the guideline. The rationale for this approach is explained in section 6.7 and in recommendation 3. Although there is no danger of tissue hypoxia at any saturation above $90 \%$, a drop of oxygen saturation below $94 \%$ may indicate deterioration and should prompt a further assessment. Supplementary oxygen should be started using nasal cannulae at $2-4 \mathrm{l} / \mathrm{min}$ or a simple face mask at $5 \mathrm{l} / \mathrm{min}$ or $35-40 \%$ Venturi mask and adjusted as necessary to maintain a saturation of $94-98 \% .{ }^{210}$ The BTS asthma guideline recommends giving high-flow oxygen to all patients with acute severe asthma. However, this aspect of the guideline was written in the early 1990s, before oximetry was in routine use. A study which was published in 2003 showed that the administration of $100 \%$ oxygen to patients with acute severe asthma produced an increased $\mathrm{PaCO}_{2}$ and a decreased peak expiratory flow compared with patients treated with $28 \%$ oxygen. ${ }^{211}$ The authors of that study recommended the use of targeted oxygen therapy rather than giving high concentration oxygen to all patients with acute severe asthma. It remains appropriate to give oxygen to patients with acute severe asthma in the absence of oximetry or blood gas results, but there is no evidence of benefit from giving oxygen to patients who are not hypoxaemic. Oxygen should not be withheld from hypoxaemic patients with severe asthma because of concerns about possible hypercapnia, although there is some evidence that this phenomenon does occur. ${ }^{211} 212$ Hypercapnia in acute asthma indicates a near-fatal attack and indicates the need for consideration of intensive care admission and ventilation. ${ }^{187}$

\section{Recommendation (see table 2)}

- In acute asthma, aim at an oxygen saturation of $94-98 \%$. [Grade C]

\subsubsection{Pneumonia}

The BTS guideline for pneumonia recommends aiming at an oxygen saturation above $92 \%$ and $\mathrm{PaO}_{2}>8 \mathrm{kPa}(60 \mathrm{~mm} \mathrm{Hg})$ in uncomplicated pneumonia with appropriate adjustments for patients with COPD, guided by blood gas measurements. ${ }^{213}$ The present guideline endorses these principles. For internal consistency, a saturation range of $94-98 \%$ is recommended for most adults and it is recommended that patients with COPD complicated by pneumonia should be managed in accordance with the COPD section of the present guideline.

\section{Recommendation (see table 2)}

- In cases of pneumonia, aim at an oxygen saturation of 9498\%. [Grade D]

8.11.4 Lung cancer and other cancers with pulmonary involvement Most patients with cancer who present with acute breathlessness have a specific causative factor such as a pleural effusion, pneumonia, COPD, anaemia or collapse of a lobe or of the left or right lung. ${ }^{214} 215$ One small double blind trial reported that hypoxaemic patients with advanced cancer $\left(\mathrm{SpO}_{2} \quad<90 \%\right)$ had reduced dyspnoea breathing oxygen compared with air, but a larger and more recent study failed to show benefit from oxygen compared with air, even when hypoxaemia was present. ${ }^{130} 216$ A single blind study involving 38 hospice patients with dyspnoea at rest showed a reduction in breathlessness when oxygen or air was given. ${ }^{217}$ Other studies have shown improvements in breathlessness in patients with cancer given opiates or benzodiazepines but not with oxygen. ${ }^{131} 132215218219$ A systematic review of oxygen and air flow on the relief of dyspnoea at rest in patients with advanced disease of any cause found low-grade scientific evidence that oxygen and airflow improve dyspnoea in some patients with advanced disease at rest. ${ }^{134}$ This systematic review could only find evidence involving a total of 83 patients and most were hypoxaemic and already receiving oxygen therapy. Based on the existing evidence, it is likely that cancer patients with significant hypoxaemia may have some relief from breathlessness if given oxygen, but there is no evidence for any benefit in patients who are breathless but not hypoxaemic, and there is evidence that opiates are effective in palliating breathlessness in this group of patients. In addition to specific management of the causative factor, oxygen should be given to maintain a saturation of $94-98 \%$ except for patients with co-existing COPD who should be treated in accordance with the COPD section of this guideline. Monitoring of oxygen saturation is not necessary when the patient is in the last few days of life.

\section{Recommendations (see table 2)}

- In breathlessness due to lung cancer, oxygen therapy may be beneficial and a trial of oxygen therapy is recommended. Aim at an oxygen saturation of $94-98 \%$ unless there is coexisting COPD. However, monitoring of oxygen saturation is not necessary when the patient is in the last few days of life. [Grade D] 
8.11.5 Deterioration of fibrotic lung conditions and other conditions involving parenchymal lung disease or alveolitis

It is recognised that patients with fibrosing lung conditions such as idiopathic pulmonary fibrosis may have acute deteriorations or exacerbations, often during intercurrent chest infections. Other patients may present acutely with breathlessness due to extrinsic allergic alveolitis, sarcoidosis or other types of parenchymal lung disorders. These patients often have a high degree of $\mathrm{V} / \mathrm{O}$ mismatch and a requirement for high oxygen concentrations to achieve satisfactory blood gases and they are not at risk of hypercapnia. It is recommended that treatment is started with $60 \%$ oxygen from a Venturi mask or $6 \mathrm{l} / \mathrm{min}$ via nasal cannulae if the patient can tolerate a high nasal flow rate. The oxygen level should be adjusted upwards or downwards to maintain an oxygen saturation in the range of $94-98 \%$, but this level may not be achievable or only achievable with a reservoir mask. Patients with end-stage pulmonary fibrosis are rarely suitable for invasive or non-invasive ventilation because of the progressive nature of the condition.

\section{Recommendation (see table 2)}

- In acute deterioration of pulmonary fibrosis or other parenchymal lung diseases, aim at an oxygen saturation of 94-98\% or the highest possible if these targets cannot be achieved. [Grade D]

\subsubsection{Pneumothorax}

As with pleural effusions, patients with a large pneumothorax may be breathless and hypoxaemic and may require supplementary oxygen for symptom relief pending definitive treatment by aspiration or drainage. However, high concentration inhaled oxygen can also increase the rate of reabsorption of air from a pneumothorax up to fourfold. ${ }^{74}$ For this reason, the BTS guideline on the management of pneumothorax recommends the use of high concentration oxygen (reservoir mask) in all non-COPD patients who require hospital admission for observation due to a moderate-sized pneumothorax that does not require drainage. ${ }^{75}$ Once a pneumothorax is drained or aspirated successfully, the patient should not require oxygen therapy unless there is additional pathology such as pneumonia, asthma or COPD requiring specific treatment.

\section{Recommendations (see table 2)}

- In most cases of pneumothorax, aim at an oxygen saturation of $94-98 \%$ if the patient is at risk of hypercapnic respiratory failure. [Grade D]

- In patients having hospital observation without drainage, the use of high concentration oxygen $(15 \mathrm{l} / \mathrm{min}$ flow rate via reservoir mask) is recommended. [Grade $\mathbf{C}$ ]

\subsubsection{Pleural effusion}

If a pleural effusion is causing significant breathlessness, the most effective treatment is to drain the effusion (but not too quickly in view of the risk of re-expansion pulmonary oedema). Hypoxaemic patients with pleural effusions are likely to benefit from supplementary oxygen therapy. The BTS guidelines for management of pleural effusions do not give any specific advice concerning oxygen therapy, but it seems reasonable to give supplementary oxygen to hypoxaemic patients to maintain a saturation of $94-98 \%$.

\section{Recommendation (see table 2)}

- In pleural effusion, aim at an oxygen saturation of $94-98 \%$ (or $88-92 \%$ if the patient is at risk of hypercapnic respiratory failure). [Grade D]

\subsubsection{Pulmonary embolism}

Most patients with suspected pulmonary embolism have normal oxygen saturation and the main focus of treatment is to reach a specific diagnosis and to commence anticoagulant treatment. These patients do not require oxygen therapy unless there is hypoxaemia. In these cases, the lowest dose of oxygen that will achieve a target saturation of $94-98 \%$ is recommended. However, patients with massive or multiple pulmonary embolism may be profoundly hypoxaemic and should initially be given high concentration oxygen via a reservoir mask to achieve an oxygen saturation of $94-98 \%$ pending definitive treatment such as thrombolysis. It has been suggested that the blood oxygen saturation may underestimate the severity of pulmonary artery obstruction in acute pulmonary embolism if shock is present. ${ }^{220}$

\section{Recommendation (see table 2)}

- In pulmonary embolism, aim at an oxygen saturation of 94$98 \%$ or $88-92 \%$ if the patient is at risk of hypercapnic respiratory failure. [Grade D]

\subsubsection{Acute heart failure}

Most patients with acute heart failure are breathless, usually due to pulmonary oedema or low cardiac output, especially if cardiogenic shock is present. The pathophysiology of oxygen transport in cardiogenic shock has been discussed in detail by Creamer and colleagues. ${ }^{221}$ It has been shown in an animal model that the ventilatory failure of cardiogenic shock may be due to an impairment of the contractile process of the respiratory muscles. ${ }^{222}$

In addition to specific treatment for heart failure, patients should be given supplementary oxygen to maintain a saturation of $94-98 \%$. This is consistent with the European Society of Cardiology Task Force and European Society of Intensive Care recommendation that patients with acute heart failure should receive oxygen to maintain $\mathrm{SpO}_{2}$ of $92-96 \% .{ }^{223}$ It is reasonable to initiate treatment with $40 \%$ or $60 \%$ oxygen for hypoxaemic patients with heart failure, followed by upward or downward adjustment to maintain saturation in the desired range. Patients with marked hypoxaemia (saturation $<85 \%$ ) should be treated with a reservoir mask initially and patients with co-existing COPD will require a lower target saturation of $88-92 \%$ pending the availability of blood gas results.

In hospital settings, patients with acute pulmonary oedema may benefit from continuous positive airway pressure and from non-invasive ventilatory support. ${ }^{224-227}$

\section{Recommendations (see table 2)}

- In acute heart failure, aim at an oxygen saturation of 94$98 \%$ or $88-92 \%$ if the patient is at risk of hypercapnic respiratory failure. [Grade D]

- Consider treatment with continuous positive airway pressure if there is hypoxaemia and treatment with non-invasive ventilation (BiPAP) if there is co-existent hypercapnia. [Grade C] 


\subsubsection{Postoperative breathlessness or hypoxaemia on general surgical wards}

These guidelines do not cover immediate postoperative care in post-anaesthetic recovery units, high dependency units or intensive care units (ICUs). Some recent trials have shown a reduced incidence of wound infection when high-dose oxygen was given perioperatively to patients having bowel surgery but not general surgery. ${ }^{79-81}$ This planned use of oxygen postoperatively is also outside the scope of this guideline.

There is some controversy about the use of "routine" supplemental oxygen postoperatively and no good evidence supporting such a policy. . $^{153}$ 228-230 The SIGN guideline on postoperative care recommends supplemental oxygen therapy for certain high-risk groups such as those with coronary artery disease, obesity, thoracic and upper abdominal surgery, but acknowledges lack of evidence to support these suggestions and does not specify an oxygen dose or target saturation for such patients. ${ }^{229}$ This SIGN guideline recommends maintaining an oxygen saturation above $92 \%$ for postoperative patients, which fits well with the suggested target saturation in the present guideline of $94-98 \%$ for most patients who require supplementary oxygen therapy.

Patients on general surgical wards can develop sudden breathlessness or hypoxaemia due to a variety of postoperative complications such as pneumonia, pulmonary embolism, opiate analgesia and atelectasis. The use of oxygen for specific postoperative complications such as pneumonia should follow the guidance for each condition (for most patients the target will be 94-98\%). Special care must be taken in cases of COPD and other risk factors for hypercapnic respiratory failure. Management of these cases can be enhanced by early specialist referral or the input of expert assistance from ICU Outreach Teams. These cases should be identified as being at risk during preoperative assessment and a target saturation of $88-92 \%$ is suggested pending the availability of blood gas results.

\section{Recommendations (see table 2)}

- For postoperative surgical patients, aim at a saturation of $94-98 \%$ or $88-92 \%$ if at risk of hypercapnic respiratory failure. [Grade D]

- For postoperative surgical patients with COPD or other risk factors for hypercapnic respiratory failure, aim at a saturation of $88-92 \%$ pending results of blood gas analysis. If the $\mathrm{PaCO}_{2}$ is normal, adjust target range to $94-98 \%$ and repeat blood gas measurements after 30-60 min (see table 3 and chart 1 (fig 1)).

\subsubsection{Breathlessness due to severe anaemia}

If breathlessness is due to severe anaemia, the specific treatment is blood transfusion. Studies by Canadian researchers in the late 1990s have shown that haemoglobin levels of $70 \mathrm{~g} / 1$ (7 g/dl) were as safe as higher levels and may produce fewer complications in the critically ill. ${ }^{55}$ However, this study was conducted using non-leucocyte-depleted blood and it is possible that some of the infective complications in the group who were given more transfusions might have been avoided by the use of leucocyte-depleted blood. The optimal transfusion target for critically ill patients therefore remains the subject of ongoing discussion among experts in critical care medicine (section 5.6.2). Giving oxygen to increase an already normal oxygen saturation will have very little effect on the oxygen-carrying power of the blood, but it is reasonable to administer supplemental oxygen to maintain a saturation of $94-98 \%$ (if the saturation is below these levels breathing air of if breathlessness is a very prominent symptom).

\section{Recommendations (see table 2)}

- In anaemia, aim at an oxygen saturation of $94-98 \%$ or $88-$ $92 \%$ if the patient is at risk of hypercapnic respiratory failure. [Grade D]

- Give packed red cells if the haemoglobin level falls below 70 $80 \mathrm{~g} / \mathrm{l}(7-8 \mathrm{~g} / \mathrm{dl})$ in most cases or $100 \mathrm{~g} / \mathrm{l}(10 \mathrm{~g} / \mathrm{dl})$ if the patient has unstable or symptomatic ischaemic heart disease. [Grade B]

\subsubsection{Sickle cell crisis}

Patients with sickle cell disease frequently present with an acute painful crisis and less frequently with an "acute chest syndrome" comprising breathlessness, chest pain and fever with pulmonary infiltrates on the chest radiograph. The exact causes and mechanisms are not well understood, but oxygen should be given to all hypoxaemic patients with sickle cell crisis to avoid further intravascular sickling. There are no randomised studies of oxygen therapy in acute chest syndrome and no randomised studies of acute painful crisis in adults, but two small randomised trials showed no clinical benefit in nonhypoxaemic children with acute painful crisis. ${ }^{231} 232$ Patients with sickle cell disease may have a reduced oxygen saturation even when clinically stable. Homi and colleagues reported a mean saturaton of only $92.5 \%$ (95\% CI $92.0 \%$ to $93.0 \%$ ) in a group of children and young adults (age 9-18 years) with stable sickle cell disease compared with an average saturation of $97.1 \%$ (95\% CI 98.8\% to 97.3\%) in a local control group. ${ }^{233}$ The British Committee for Standards in Haematology have recommended that oxygen should be given if the oxygen saturation falls below what is normal for the individual patient or a default target of $95 \%$ if the usual saturation is unknown. ${ }^{234}$ This is consistent with the advice in the present guideline to aim at a normal or near-normal oxygen saturation for non-hypoxaemic patients with a target saturation of $94-98 \%$. Readers are referred to the guideline on sickle cell disease for disease-specific management of this condition. ${ }^{234}$

\section{Recommendation (see table 2)}

- In sickle cell crisis and acute chest syndrome, aim for an oxygen saturation of $94-98 \%$ or aim at the saturation level that is usual for the individual patient. [Grade B]

\subsection{Recommended oxygen therapy for patients who may be} vulnerable to medium or high doses of oxygen (see also table 3 )

COPD is the best known condition that can predispose to hypercapnic (type 2) respiratory failure with acidosis, especially if the blood oxygen level is increased above $10 \mathrm{kPa}(75 \mathrm{~mm} \mathrm{Hg}) \cdot{ }^{34} 235$ However, there are a number of other conditions which can render patients vulnerable to hypercapnic respiratory failure. The emphasis for such patients is to avoid clinically harmful levels of hypoxaemia or hypercapnia by giving carefully titrated oxygen therapy or, if necessary, by supporting the patient with the use of non-invasive or invasive mechanical ventilation.

Non-COPD patients at risk of hypercapnic respiratory failure include the following:

- Cystic fibrosis.

Non-CF bronchiectasis (often in association with COPD or severe asthma)

- Severe kyphoscoliosis or severe ankylosing spondylitis. 
- Severe lung scarring from old tuberculosis (especially with thoracoplasty).

- Morbid obesity (body mass index $>40 \mathrm{~kg} / \mathrm{m}^{2}$ ).

- Musculoskeletal disorders with respiratory muscle weakness, especially if on home ventilation.

- Overdose of opiates, benzodiazepines or other respiratory depressant drugs.

\subsubsection{COPD exacerbations}

There is an extensive literature documenting the effects of highdose oxygen therapy in acute COPD. ${ }^{34} 656694102106118$ 235-251 These reports show that the administration of supplemental oxygen to patients with exacerbated COPD often causes a rise in $\mathrm{PaCO}_{2}$ with subsequent respiratory acidosis for reasons summarised in sections 5.3, 5.4 and 6.3.1. The literature is summarised in detail in the review by Murphy et al. ${ }^{10}$ Some patients with COPD are prone to repeated episodes of hypercapnic respiratory failure and others may not ever suffer from this complication. Even among patients with COPD with chronic hypercapnia, not all will develop an increased carbon dioxide level (and acidosis) during acute exacerbations. Apart from patients with recurrent hypercapnic respiratory failure, it is not possible to predict if individual patients with COPD will develop hypercapnia during an acute exacerbation, so all patients with moderate or severe COPD should be considered to be at risk of this complication until the results of blood gas measurements are available. It is therefore essential that patients who are at risk of having COPD should be diagnosed accurately, and this can only be done by measurement of $\mathrm{FEV}_{1}{ }^{25}$

Patients with acute severe exacerbations of COPD may be too breathless to undertake spirometry on arrival in hospital, but many patients are able to perform spirometry on arrival in hospital and all patients should have the test performed before discharge from hospital to confirm the diagnosis of COPD and to assess the severity of the condition. There is very little literature describing the effects of oxygen therapy in the other conditions listed above, but they are recognised to be at risk of hypercapnic respiratory failure and should be treated in a manner analogous to patients with COPD.

It has been shown that patients with COPD with a $\mathrm{pH}$ reading $<7.35\left(\left[\mathrm{H}^{+}\right]>45 \mathrm{nmol} / \mathrm{l}\right)$ despite controlled oxygen therapy are more likely to die and more likely to meet criteria for intubation and ventilation. ${ }^{34} 237241$ One of these reports also showed that patients with a high $\mathrm{PaO}_{2}$ on arrival in hospital $(>10.0 \mathrm{kPa}$ or $75 \mathrm{~mm} \mathrm{Hg}$ ) were more likely to meet criteria for ventilation and the severity of acidosis was related to high $\mathrm{PaO}_{2}$ values. ${ }^{34}$ Based on these results, Plant and colleagues recommended an upper limit of about $92 \%$ saturation for patients with exacerbations of $\mathrm{COPD}$ to prevent the $\mathrm{PaO}_{2}$ rising above $10 \mathrm{kPa}^{34}$ This report was supported by the recent work of Joosten et al which showed that a $\mathrm{PaO}_{2}$ of $>74.5 \mathrm{~mm} \mathrm{Hg}$ $(10 \mathrm{kPa})$ in acute COPD was associated with an increased likelihood of admission to a high dependency unit, increased need for non-invasive ventilation and a longer stay in hospital. ${ }^{235}$ Consequently, the guideline group has recommended a maximum saturation of $92 \%$ while awaiting blood gas results in acute exacerbations of COPD and other conditions that may predispose to type 2 respiratory failure. Although the rise in $\mathrm{PaCO}_{2}$ (and fall in $\mathrm{pH}$ ) is greatest in patients who are given sufficient oxygen therapy to elevate the $\mathrm{PaO}_{2}$ above $10 \mathrm{kPa}$, it is important to note that hypercapnia can occur in acute COPD even if the oxygen saturation is $<88 \%{ }^{249}$
The best management strategy for persistently acidotic COPD patients is a trial of non-invasive ventilation with supplementary oxygen therapy. ${ }^{25} 3234$

Some patients with previous hypercapnic respiratory failure will have alert cards or an entry in their electronic record to alert the emergency team to the optimal dose of oxygen required during the patient's previous hospital admissions (see section 9.7). In the absence of such information, it is suggested that a target of $88-92 \%$ should be set initially for patients with a history of previous non-invasive or invasive ventilation and, if necessary, modified later based on blood gas results. These patients should be categorised as very urgent by ambulance teams and emergency services, requiring immediate blood gas measurement and senior assessment on arrival at the hospital emergency department.

Unfortunately, many clinical studies have shown that patients with COPD are frequently given very high doses of oxygen, either because of misdiagnosis or because the risks of hyperoxia in patients with COPD have been overlooked. ${ }^{34} 235-237$ Many patients with COPD are unaware of the diagnosis or are mislabelled as having asthma (see section 9.5).

The consensus from the literature is that patients with acute exacerbations of COPD should be treated with Venturi masks to minimise the risks of hypercapnic respiratory failure and to achieve a high gas flow from the mask in patients with a high inspiratory flow rate. ${ }^{10}$ It is not yet known if it is better to start with a $28 \%$ Venturi mask or a $24 \%$ Venturi mask. Management with a 28\% Venturi mask appears to be safe. ${ }^{252}$ The current guideline recommends starting with a $28 \%$ Venturi mask in cases of COPD with no known history of hypercapnic respiratory failure, with downward adjustment to a $24 \%$ mask (in hospital) if the saturation rises above $92 \%$. In cases of prior hypercapnic failure who do not have an oxygen alert card, it is recommended that prehospital treatment should be commenced using a $28 \%$ Venturi mask at $4 \mathrm{l} / \mathrm{min}$ or a $24 \%$ Venturi mask in hospitals with a target saturation of $88-92 \%$. Observational studies in the $1960 \mathrm{~s}$ suggested that a $\mathrm{PaO}_{2}$ of $50 \mathrm{~mm} \mathrm{Hg}$ or $6.7 \mathrm{kPa}$ (saturation about $84 \%$ ) will prevent death from hypoxaemia in acute COPD exacerbations. ${ }^{242} 243$ If the saturation should fall below $88 \%$ despite treatment with a $24 \%$ or $28 \%$ Venturi mask, the patient should be treated with nasal cannulae or a simple face mask with the flow adjusted to maintain a saturation of $88-92 \%$ pending the availability of blood gas results. This small subgroup of patients is at very high risk of death and should be treated as a high priority on arrival in emergency departments, requiring immediate senior assessment and arterial blood gas measurements.

- Measurement of $\mathrm{FEV}_{1}$ may confirm (or exclude) a diagnosis of airflow obstruction and the $\mathrm{FEV}_{1}$ level is a useful indicator of disease severity in COPD. ${ }^{25}$ [Evidence level III]

- Patients with exacerbations of COPD are at risk of hypercapnic (type 2) respiratory failure with respiratory acidosis. [Evidence level IIa]

- The risk of respiratory acidosis in patients with hypercapnic respiratory failure is increased if the arterial oxygen tension is above $10.0 \mathrm{kPa}$ due to previous excessive oxygen use. [Evidence level IIa]

- These patients with chronic lung disease are usually "acclimatised" to living with an oxygen saturation which may be in the high 80s or low 90s and there is not likely to be any benefit from increasing the saturation above these levels during acute illness. [Evidence level III] 


\section{Recommendations (see table 3)}

- If the diagnosis is unknown, patients over 50 years of age who are long-term smokers with a history of chronic breathlessness on minor exertion such as walking on level ground and no other known cause of breathlessness should be treated as if having COPD for the purposes of this guideline. Patients with COPD may also use terms such as chronic bronchitis and emphysema to describe their condition but may sometimes mistakenly use "asthma". FEV 1 should be measured on arrival in hospital if possible and should be measured at least once before discharge from hospital in all cases of suspected COPD. [Grade D]

- Patients with a significant likelihood of severe COPD or other illness that may cause hypercapnic respiratory failure should be triaged as very urgent on arrival in hospital emergency departments and blood gases should be measured on arrival in hospital. [Grade D]

- Prior to availability of blood gas measurements, use a $28 \%$ Venturi mask at $4 \mathrm{1} / \mathrm{min}$ or $24 \%$ Venturi mask at $2 \mathrm{l} / \mathrm{min}$ and aim for an oxygen saturation of $88-92 \%$ for patients with risk factors for hypercapnia but no prior history of type 2 respiratory failure. [Grade D]

- For patients with known previous hypercapic respiratory failure but no oxygen alert card, aim at a saturation of 88$92 \%$ until the results of blood gas measurements are available (see recommendation 5 ).

- If the saturation remains below $88 \%$ in prehospital care despite a $28 \%$ Venturi mask, change to nasal cannulae at 2$6 \mathrm{l} / \mathrm{min}$ or a simple face mask at $5 \mathrm{l} / \mathrm{min}$ with target saturation of $88-92 \%$ and alert the $A \& E$ department that the patient is to be treated as a high priority. [Grade D]

- Patients with a respiratory rate $>30$ breaths/min should have the flow rate set to $50 \%$ above the minimum flow rate specified for the Venturi mask and/or packaging. Increasing the oxygen flow rate into a Venturi mask does not increase the concentration of oxygen which is delivered (see recommendation 32 ).

- Aim at a prespecified target saturation range (if available) in patients with a history of previous respiratory acidosis. In many cases the ideal target saturation will be specified on the patient's alert card. If no information is available, aim at a saturation level of $88-92 \%$ pending blood gas results.

\section{[Grade D]}

- Patients with prevous hypercapnic respiratory failure should have a personalised oxygen alert card and this information should be available to primary care staff, ambulance staff and hospital staff (see recommendations 23-25).

- If following blood gas measurements the $\mathrm{pH}$ and $\mathrm{PCO}_{2}$ are normal, aim for an oxygen saturation of $94-98 \%$ unless there is a history of previous hypercapnic respiratory failure requiring non-invasive ventilation or intermittent positive pressure ventilation. [Grade D]

- Recheck blood gases after 30-60 min (or if there is evidence of clinical deterioration) for all patients with COPD or other risk factors for hypercapnic respiratory failure even if the initial $\mathrm{PaCO}_{2}$ measurement was normal. [Grade D]

- If the $\mathrm{PaCO}_{2}$ is raised but $\mathrm{pH}$ is $\geqslant 7.35\left(\left[\mathrm{H}^{+}\right] \leqslant 45 \mathrm{nmol} / \mathrm{l}\right)$, the patient has probably got long-standing hypercapnia; maintain target range of $88-92 \%$ for these patients. Blood gas measurements should be repeated at 30-60 min to check for rising $\mathrm{PaCO}_{2}$ or falling $\mathrm{pH}$. [Grade D]

- If the patient is hypercapnic $\left(\mathrm{PaCO}_{2}>6 \mathrm{kPa}\right.$ or $\left.45 \mathrm{~mm} \mathrm{Hg}\right)$ and acidotic ( $\mathrm{pH}<7.35$ or $\left[\mathrm{H}^{+}\right]>45 \mathrm{nmol} / \mathrm{l}$ ), consider noninvasive ventilation, especially if the acidosis has persisted for more than 30 min despite appropriate therapy. [Grade A]

- Once patients have stabilised, consider changing from Venturi mask to nasal cannulae at 1-2 1/min (see recommendation 31).

\subsubsection{Exacerbation of cystic fibrosis}

Patients with breathlessness due to cystic fibrosis should be managed in a Cystic Fibrosis Centre unless this is not possible for geographical reasons. If not possible, all cases should be discussed with the Cystic Fibrosis Centre or managed according to a protocol that has been agreed with the regional centre. Patients with advanced cystic fibrosis may suffer from exacerbations which are similar to exacerbations of advanced COPD with associated hypoxaemia and hypercapnia. The principles of management are similar to those in acute exacerbations of COPD, including a need to maintain adequate oxygen saturation and avoiding excessive hypercapnia and acidosis. As in COPD, non-invasive ventilation may be of value in severe cases. ${ }^{253}$ Non-invasive ventilation in cystic fibrosis may also be helpful to reduce symptoms (eg, work of breathing and dyspnoea) and assist in airway clearance.

It is recommended that patients with acute exacerbations of cystic fibrosis should be managed on similar lines to patients with acute exacerbations of COPD with a target oxygen saturation of $88-92 \%$ for most patients, but recognition that individual patients may need to be managed differently on the basis of previous and current blood gas measurements. One study has shown that patients with a respiratory rate above 30 breaths/min often have an inspiratory flow rate above the minimum flow rate specified on the mask packaging. ${ }^{254}$ However, there is no direct experimental evidence of the clinical effectiveness of increased flow rates from Venturi devices. It is possible that patients with very high inspiratory flow rates might benefit from a $28 \%$ Venturi mask with the flow rate set at $6-8 \mathrm{l} / \mathrm{min}$ to minimise the risk of the inspiratory flow rate exceeding the gas flow rate (see table 10 in section 10). Patients with cystic fibrosis who have had previous episodes of hypercapnic respiratory failure should be issued with an oxygen alert card with recommendations based on previous blood gas measurements (see recommendations 23-25).

\section{Recommendation (see table 3)}

- Initial treatment of cystic fibrosis exacerbations should be similar to the initial treatment of COPD exacerbations (see section 8.12.1). [Grade D]

\subsubsection{Chronic musculoskeletal and neurological disorders}

Hypoxaemia due to musculoskeletal and neurological disorders is usually associated with acute illness (such as a chest infection) superimposed on a chronic neuromuscular condition. However, muscle weakness can be acute or subacute (eg, Guillain-Barré syndrome, see section 8.13.7). For most patients with inadequate ventilation due to neuromuscular weakness, non-invasive or invasive ventilatory support is more useful than supplementary oxygen and these patients are at risk of hypercapnic respiratory failure which may be aggravated by high doses of oxygen. For this reason it is recommended that spirometry should be monitored carefully and blood gases should be obtained as early as possible in all such cases. Pending the availability of blood gas results, a saturation target of 88$92 \%$ will avoid the risks of severe hypoxaemia or severe hypercapnia. 


\section{Recommendation (see table 3)}

- In the initial management of musculoskeletal and neurological disorders with acute respiratory failure, aim at an oxygen saturation of $88-92 \%$. Many such patients will be suitable for non-invasive ventilation. [Grade D]

\subsubsection{Obesity-hypoventilation syndrome}

Patients with the obesity-hypoventilation syndrome often develop chronic hypercapnic respiratory failure and they may decompensate acutely to produce hypercapnic respiratory failure with acidosis. ${ }^{255}$ For purposes of oxygen therapy, these patients should be treated in a similar manner to patients with hypercapnic respiratory failure due to an acute exacerbation of COPD (but they clearly do not require bronchodilator and steroid therapy). The initial target saturation will usually be 88$92 \%$ but, as with COPD, a lower target range may be appropriate for individual patients based on blood gas measurements during a previous exacerbation or due to acute acidosis. Assessment of patients with increasing shortness of breath or worsening oxygen saturation must include blood gases. As in COPD, patients with respiratory acidosis may benefit from noninvasive ventilation.

\section{Recommendations (see table 3)}

- In the initial management of the obesity-hypoventilation syndrome with acute exacerbation, aim at an oxygen saturation of $88-92 \%$. [Grade D]

- Non-invasive ventilation should be considered for all of the above groups of patients if the $\mathrm{pH}$ is $<7.35$ or $\left[\mathrm{H}^{+}\right]$ $>45 \mathrm{nmol} / \mathrm{l}$. [Grade C]

\subsection{Common medical emergencies for which oxygen therapy is indicated only if hypoxaemia is present (see also table 4)}

There are a number of conditions such as myocardial infarction, angina and stroke for which oxygen was traditionally given to all patients in an attempt to increase oxygen delivery to the heart or brain. However, the administration of supplemental oxygen to normoxaemic patients has very little effect on blood oxygen content but may reduce myocardial and cerebral blood flow due to vasoconstriction which is a physiological response to hypoxia in most organs. There is no evidence of benefit from the administration of supplemental oxygen to non-hypoxaemic patients with these conditions and there is some evidence of possible harm, so it is recommended that oxygen should only be given to patients with these conditions if hypoxaemia is present, usually due to complications such as heart failure or pneumonia. There are no published trials supporting the use of oxygen to relieve breathlessness in non-hypoxaemic patients, and there is evidence from randomised studies that oxygen does not relieve breathlessness compared with air in non-hyoxaemic COPD patients who are breathless following exertion. ${ }^{129}$

\subsubsection{Acute myocardial infarction, suspected myocardial infarction and acute coronary syndromes}

Some patients with acute myocardial infarction have heart failure and should be treated accordingly (see section 8.11.9). Most patients with suspected or confirmed myocardial infarction are not hypoxaemic and most are not breathless. In the case of non-hypoxaemic patients, it is not known if supplementary oxygen may be beneficial by increasing the amount of oxygen delivered to the hypoxaemic area of myocardium or whether it may actually cause vasoconstriction with increased systemic vascular resistance and reduced myocardial oxygen supply with worsened systolic myocardial performance. ${ }^{256-264}$ A recent study of patients having coronary arteriography found that breathing $100 \%$ oxygen reduced coronary blood flow velocity by $20 \%$ and increased coronary resistance by $23 \%{ }^{265}$ There is also a theoretical possibility that high oxygen levels might exacerbate reperfusion injury to the heart. ${ }^{263}$ Despite a multitude of large studies of intervention in myocardial infarction, there has been only one randomised study of oxygen therapy (in 1976) and this study did not identify any benefit from such therapy but found some evidence of potential harm. ${ }^{107}$ This trial reported a significantly greater rise in myocardial enzyme in the oxygen group, suggesting a greater infarct size. There was a threefold increase in mortality on oxygen therapy that did not reach statistical significance ( 3 deaths in 77 patients treated with air versus 9 deaths in 80 patients given oxygen at $6 \mathrm{l} / \mathrm{min}$ via a simple face mask for $24 \mathrm{~h}$ ). A systematic review and a historical review of oxygen therapy in acute myocardial ischaemia have both concluded that there was no evidence to support this practice in non-hypoxaemic patients and some evidence of possible harm. ${ }^{266} 267$ One study from 1969 showed that hypoxia did not affect the availability of oxygen for myocardial metabolism in normal subjects until the oxygen saturation fell to about $50 \%$, but evidence of myocardial ischaemia was seen at saturations of $70-85 \%$ in subjects with coronary artery disease. $^{268}$ In these circumstances it is advised that patients with myocardial infarction or chest pain suspicious of myocardial infarction should be given supplementary oxygen if required to maintain a saturation of $94-98 \%$.

The study by Lal and colleagues ${ }^{269}$ in the 1960 s showed that hypoxaemia was present in a high proportion of patients diagnosed with myocardial infarction and could usually be reversed by medium-dose oxygen, but sometimes required treatment with a reservoir mask to achieve a $\mathrm{PaO}_{2}$ oxygen tension $>60 \mathrm{~mm} \mathrm{Hg}(8 \mathrm{kPa})$. The study by Wilson and Channer $^{2}$ in 1997 showed that desaturation below 90\% was common in patients with myocardial infarction within the first $24 \mathrm{~h}$ of admission to a coronary care unit, but these authors may not have been aware that nocturnal desaturation to this level is very common in healthy individuals. ${ }^{26}$ Wilson and Channer did not demonstrate any correlation between hypoxaemic events and adverse cardiac events. ${ }^{2}$ They did, however, show that monitoring by oximetry was inadequate in UK coronary care units in the mid 1990s.

There are no UK guidelines for oxygen therapy in acute myocardial infarction. The 1998 European Society for Cardiology/European Resuscitation Council Task Force recommended the use of $3-5 \mathrm{l} / \mathrm{min}$ oxygen via face mask to all patients with chest pain of presumed cardiac origin, but no evidence was presented to support this advice. ${ }^{270}$ However, most of the papers that have raised concerns about the effects of oxygen on myocardial blood flow have been published since that date (see preceding paragraph). The European Society of Cardiology published subsequent guidance on the management of ST elevation myocardial infarction in $2003 .{ }^{271}$ This revised guidance recommended the use of oxygen at $2-41 / \mathrm{min}$ by mask or nasal cannulae for patients with heart attacks associated with breathlessness or heart failure. The 2007 SIGN guideline for acute coronary syndromes states that there is no evidence that routine administration of oxygen to all patients with acute coronary syndromes improves clinical outcome or reduces infarction size. ${ }^{272}$ The SIGN guideline gives a grade $\mathrm{D}$ recommendation that oxygen should be administered to patients with hypoxaemia, pulmonary oedema or continuing myocardial ischaemia. 
The European Resuscitation Council Guidelines for the management of acute coronary syndromes in 2005 recommended the use of supplementary oxygen at 4-8 1/min (device not specified) for patients with arterial oxygen saturation $<90 \%$ and/or pulmonary congestion. ${ }^{273}$ The guideline acknowledged the lack of evidence of benefit for non-hypoxaemic patients but recommended supplementary oxygen in case of unrecognised hypoxaemia. This situation might apply in the prehospital setting but not in the hospital setting. The limited available evidence therefore supports the suggestion that clinicians should aim at normal or near-normal oxygen saturation in patients with myocardial infarction, acute coronary syndrome and chest pain suspicious of coronary artery disease. A target saturation range of $94-98 \%$ will meet all of these goals, and further research of this topic should be prioritised because this is such a common medical problem and there is so little existing evidence. Most 999 calls to ambulance services because of chest pain are currently treated with high concentration oxygen in accordance with the Joint Royal Colleges Ambulance Liaison Committee (JRCALC) guidance. ${ }^{274}$ However, most such patients have a final diagnosis of undifferentiated chest pain rather than acute coronary artery syndrome and most patients with undifferentiated chest pain are normoxaemic. The clinical management of a very large number of patients will therefore be changed following the introduction of this guideline.

\section{Recommendation (see table 4)}

- In myocardial infarction and acute coronary syndromes, aim at an oxygen saturation of $94-98 \%$ or $88-92 \%$ if the patient is at risk of hypercapnic respiratory failure. [Grade D]

\subsubsection{Stroke}

In the past it was customary to give supplementary oxygen to all patients with stroke to try to improve cerebral oxygenation. However, there has been only one randomised trial of oxygen therapy in stroke. ${ }^{108}$ This trial found no difference in 1-year survival for the entire cohort of patients with stroke and no difference in survival for patients with more severe strokes. However, for patients with minor or moderate strokes, 1-year mortality was $18 \%$ in the group given oxygen and $9 \%$ in the group given air (OR 0.45; 95\% CI 0.23 to $0.90, p=0.023$ ). Based largely on the results of this trial, the Royal College of Physicians stroke guideline recommends that oxygen saturation should be maintained in the normal range in patients with stroke. ${ }^{275}$ It is recommended that patients with stroke should receive supplementary oxygen only if this treatment is required to achieve an oxygen saturation of $94-98 \%$ (88-92\% for patients with co-existing risk of COPD or other risk of respiratory acidosis)

There has also been some discussion concerning the optimal body position for the management of patients with stroke and potential hypoxaemia. A systematic review concluded that there was limited evidence that sitting in a chair had a beneficial effect and lying positions had a deleterious effect on oxygen saturation in patients with acute stroke with respiratory comorbidities, but patients with acute stroke without respiratory co-morbidities can adopt any body position. ${ }^{136}$ The authors of this review recommended that people with acute stroke and respiratory co-morbidities should be positioned as upright as possible.

\section{Recommendation (see table 4)}

- In stroke, aim at an oxygen saturation of $94-98 \%$ or $88-92 \%$ if the patient is at risk of hypercapnic respiratory failure. [Grade B]

\subsubsection{Obstetric emergencies and labour}

The use of oxygen has been recommended during many obstetric emergencies and, in particular, for collapse related to haemorrhage, pulmonary embolism, eclampsia or amniotic fluid embolism. Severe pre-eclampsia and eclampsia may occasionally present with pulmonary oedema and this can occur in the antenatal or postnatal periods. Medical problems such as pneumonia or acute exacerbations of asthma are not uncommon during pregnancy. Peripartum cardiomyopathy is rare but may present with heart failure in the postnatal period. Major trauma is increasingly common, particularly related to road traffic accidents.

The use of oxygen during pregnancy should follow the same general principles as the use of oxygen for other patients. Pregnant women suffering major trauma or severe hypoxaemia should be started on high concentration oxygen via a nonrebreathing reservoir mask and those with milder hypoxaemia can use nasal cannulae or a simple face mask or Venturi mask to achieve an oxygen saturation of $94-98 \%$ in most cases. If an undelivered woman is hypoxaemic, she should be managed with left lateral tilt applied. This will improve cardiac output ${ }^{276}$ and may also facilitate breathing for mechanical reasons.

Oxygen is commonly given as part of the treatment for many obstetric emergencies. However, it is recommended that, when oxygen is administered during pregnancy or labour, clinicians should aim to achieve normoxaemia (saturation 94-98\%). There is no randomised trial evidence to suggest that maternal "hyperoxaemia" is beneficial to mother or fetus.

Oxygen is often given when acute fetal compromise is suspected in labour in the hope of increasing oxygen delivery to the fetus. A Cochrane review found no trials addressing the use of oxygen for fetal compromise. However, two trials of prophylactic oxygen in labour found a significant increase in the incidence of cord blood acidosis $\left(\mathrm{pH}<7.20\right.$ or $\left[\mathrm{H}^{+}\right]$ $>63 \mathrm{nmol} / \mathrm{l}$ ) in the oxygenation group (RR 3.5 (95\% CI 1.34 to 9.19$))$. $^{277}$

It is recommended that pregnant women with evidence of hypoxaemia should have their blood oxygen saturation maintained in the normal range (94-98\%) using supplemental oxygen as necessary to achieve this effect. This applies before or during labour as well as in the postnatal period. The causes of maternal hypoxaemia may include trauma, pre-existing or de novo medical conditions as well as pregnancy-specific complications. In all of these situations the aim should be normoxaemia (saturation 94-98\%).

\section{Recommendations}

14. Women who suffer from major trauma, sepsis or acute illness during pregnancy should receive the same oxygen therapy as any other seriously ill patients, with a target oxygen saturation of 94$\mathbf{9 8 \%}$. The same target range should be applied to women with hypoxaemia due to acute complications of pregnancy (eg, collapse related to amniotic fluid embolus, eclampsia or antepartum or postpartum haemorrhage). [Grade D]

15. Women with underlying hypoxaemic conditions (eg, heart failure) should be given supplemental 
oxygen during labour to achieve an oxygen saturation of $94-98 \%$. [Grade D]

16. All women with evidence of hypoxaemia who are more than 20 weeks pregnant should be managed with left lateral tilt to improve cardiac output. [Grade B]

17. The use of oxygen during labour is widespread but there is evidence that this may be harmful to the fetus. The use of oxygen during labour is therefore not currently recommended in situations where the mother is not hypoxaemic (except as part of a controlled trial). [Grade A]

\subsubsection{Anxiety and hyperventilation or dysfunctional breathing}

Many patients who present to hospital with breathlessness are found to have no cardiopulmonary problems and many such patients have a specific diagnosis of hyperventilation, dysfunctional breathing, upper airway dysfunction or panic attacks, sometimes in addition to asthma or some other underlying respiratory disorder. ${ }^{278}$ Many such patients will have an abnormally high oxygen saturation of $99 \%$ or $100 \%$ and clearly do not require supplemental oxygen therapy. Many other nonhypoxaemic patients will present to hospital with acute breathlessness of unknown cause, and the majority of patients with an elevated respiratory rate are likely to have an organic illness. In some cases simple investigations will reveal a specific diagnosis such as pneumothorax or pneumonia or pulmonary embolism, but many cases remain undiagnosed. A policy of giving supplementary oxygen if the saturation falls below $94 \%$ will avoid exposing patients with undiagnosed medical illnesses to the risk of hypoxaemia while avoiding the unnecessary use of oxygen in patients with behavioural or dysfunctional breathlessness.

Studies in normal volunteers have demonstrated that compensatory desaturation may occur shortly after voluntary hyperventilation. ${ }^{279}$ The mean $\mathrm{PaO}_{2}$ of 10 male volunteers increased from $13.7 \mathrm{kPa} \quad(103 \mathrm{~mm} \mathrm{Hg})$ to $18.6 \mathrm{kPa}$ (140 $\mathrm{mm} \mathrm{Hg}$ ) during hyperventilation but fell to a nadir of $7.8 \mathrm{kPa}(58 \mathrm{~mm} \mathrm{Hg})$ about $7 \mathrm{~min}$ after cessation of hyperventilation and did not normalise until after a total of $17 \mathrm{~min}$ of observation. It is not known whether or not this occurs after pathological hyperventilation, but this phenomenon could cause considerable confusion if it should occur in an emergency department.

A traditional treatment for hyperventilation was to ask the subject to rebreathe from a paper bag to allow the carbon dioxide level in the blood to normalise. However, it has been shown that this practice can cause hypoxaemia with potentially fatal consequences. ${ }^{280}$ The average fall in oxygen tension during rebreathing was $26 \mathrm{~mm} \mathrm{Hg}(3.5 \mathrm{kPa})$ and the maximum fall was $42 \mathrm{~mm} \mathrm{Hg}(5.6 \mathrm{kPa})$. This guideline does not recommend rebreathing from a paper bag in cases of hyperventilation unless the patient has been shown to have hyperoxia and a low carbon dioxide level, and any such treatment should be monitored with continuous oximetry and discontinued if the patient should desaturate.

\section{Recommendations (see table 4)}

- Organic illness must be excluded before making a diagnosis of hyperventilation. [Grade C]

- Patients with a definite diagnosis of hyperventilation should have their oxygen saturation monitored. Those with normal or high $\mathrm{SpO}_{2}$ do not require oxygen therapy. [Grade B]

- Rebreathing from a paper bag can be dangerous and is NOT recommended as a treatment for hyperventilation. [Grade C]

\subsubsection{Poisoning with substances other than carbon monoxide} Many poisons and drugs can cause respiratory or cardiac depression or direct toxic effects on the lungs. The treatment of individual toxic agents is beyond the scope of this guideline. Specific antidotes such as naloxone should be given if available and oxygen saturation should be monitored closely. Supplementary oxygen should be given to achieve a target saturation of $94-98 \%$ pending the results of blood gas analysis (88-92\% if at risk of hypercapnic respiratory failure). All potentially serious cases of poisoning should be monitored in a level 2 or level 3 environment (high dependency unit or intensive care unit).

Three specific types of lung injury deserve special mention. Oxygen is known to be hazardous to patients with paraquat poisoning, ${ }^{113} 114$ and oxygen potentiates bleomycin lung injury. Because of these risks, oxygen should be given to patients with these conditions only if the oxygen saturation falls below $90 \%$. Some authors have suggested the use of hypoxic ventilation with $14 \%$ oxygen as a specific treatment for paraquat poisoning. ${ }^{281}$

Bleomycin lung injury can be potentiated by high-dose oxygen therapy, even if given several years after the initial lung injury. ${ }^{115}$ It is therefore recommended that high doses of oxygen should be avoided in patients with possible bleomycin-induced lung injury and a lower oxygen saturation target range should be accepted (eg, 88-92\%).

There is evidence from animal experiments that oxygen may potentiate lung injury from aspiration of acids. ${ }^{116} 117$ The effect in humans is not known so patients with acid inhalaton should have the usual adult target saturation range of $94-98 \%$, but it would appear prudent to aim in the lower half of the target range for these patients and clinical trials in humans are clearly required.

\section{Recommendations (see table 4)}

- In most poisonings, aim at an oxygen saturation of $94-98 \%$. [Grade D]

- In poisoning by paraquat and bleomycin, aim at a saturation of $88-92 \%$. [Grade D]

\subsubsection{Metabolic, endocrine and renal disorders}

Many metabolic and renal disorders can cause metabolic acidosis which increases respiratory drive as the body tries to correct the acidosis by increased excretion of carbon dioxide via the lungs. Although these patients have tachypnoea, they do not usually complain of breathlessness and most have a high oxygen saturation (unless there is a co-existing pulmonary or cardiac problem). Supplementary oxygen is not required for such patients unless the oxygen saturation is reduced. In such cases, oxygen should be given to maintain a saturation of 94$98 \%$.

\section{Recommendation (see table 4)}

- In most metabolic and renal disorders, aim at an oxygen saturation of $94-98 \%$. [Grade D] 
8.13.7 Acute and subacute neuromuscular disorders producing respiratory muscle weakness

Patients with acute or subacute conditons affecting the respiratory muscles are at risk of sudden onset of respiratory failure with hypoxaemia and hypercapnia and may require noninvasive or invasive ventilatory support. This applies especially to patients with Guillain-Barré syndrome for whom spirometry should be monitored carefully as this should detect the onset of severe respiratory failure prior to the development of hypoxaemia. If the oxygen level falls below the target saturation, urgent blood gas measurements should be undertaken and the patient is likely to need ventilatory support.

\section{SECTION 9: EMERGENCY USE OF OXYGEN IN AMBULANCES, COMMUNITY AND PREHOSPITAL SETTINGS}

This section applies to a range of clinical settings to include emergency oxygen use in patients' homes, GP practices or health centres and during emergency ambulance journeys to hospital. Management in some prehospital settings such as a Primary Care Centre or in a paramedic ambulance may be almost identical to hospital management. Readers are referred to section 10 for advice concerning choice of oxygen delivery devices and systems.

Readers are referred to tables $1-4$ and charts 1 and 2 (figs 1 and 2) for a summary of the key elements of oxygen therapy in common medical emergencies. A brief summary of this section can be downloaded from www.brit-thoracic.org.uk.

Ongoing care at home of chronically hypoxaemic patients is not covered by this guideline. There is little literature on which to base any recommendations when such patients have an acute exacerbation of their condition, but patient safety should be the priority. The NICE guidelines on COPD recommend that patients receiving long-term oxygen and those with an arterial $\mathrm{PO}_{2}$ of $<7 \mathrm{kPa}$ should be considered for treatment in hospital during exacerbations (recommendation 135 in NICE guideline $^{25}$ ). The BTS Emergency Oxygen Guideline Group would add that chronically hypoxaemic patients with a clinical exacerbation associated with a $3 \%$ or greater fall in oxygen saturation on their usual oxygen therapy should be assessed in hospital with blood gas estimations. Arterial $\mathrm{PO}_{2}$ of $<7 \mathrm{kPa}$ equates to $\mathrm{SpO}_{2}$ below approximately $85 \%$.

\subsection{Pulse oximetry and availability of oxygen}

It is essential to provide optimal oxygen therapy at the earliest possible opportunity while the acutely breathless patient is being assessed and treated in the community and during transfer to hospital. For most such patients the main concern is to give sufficient oxygen to support their needs. Hypoxaemia can lead to cardiac arrhythmias, renal damage and, ultimately, cerebral damage. However, excessive oxygen therapy can also be dangerous for some patients, especially those with advanced COPD. Target saturation should be used; pulse oximetry is necessary to achieve this. Section 10.4 .2 provides advice concerning the choice of oxygen cylinders in primary care practices.

Emergency ambulances and emergency/fast response type vehicles and ambulance service motorbikes and cycles should be equipped with oxygen and oximeters germane to the mode of transport. Thus, fast response cars/motorbikes and cycles will require handheld finger oximeter-type devices and staff initiating oxygen in the home will need a portable or finger oximeter. Community First Responder (CFR) schemes are encouraged to seek the opinion of the ambulance service to which they are affiliated to discuss the purchase and use of pulse oximeters Likewise Voluntary Aid Societies (VAS) medical directors are encouraged to discuss the purchase and use of pulse oximieters.

\section{Recommendations}

18. Pulse oximetry must be available in all locations where emergency oxygen is being used (see also the limitations of using pulse oximetry section 7.1.2). [Grade D]

19. Emergency oxygen should be available in primary care medical centres, preferably using oxygen cylinders with integral high-flow regulators. Alternatively, oxygen cylinders fitted with highflow regulators (delivering over $6 \mathrm{l} / \mathrm{min}$ ) must be used. [Grade D]

20. All documents which record oximetry measurements should state whether the patient is breathing air or a specified dose of supplemental oxygen. [Grade C]

\subsection{Clinical assessment by initial responder(s) (GP, nurse or ambulance team)}

It is suggested that the first healthcare professional(s) to encounter an acutely breathless patient should perform an initial "ABC" assessment (airway, breathing, circulation), followed by obtaining a quick history from the patient and/or family or friends. Immediate assessment should include a recording of pulse rate and respiratory rate and pulse oximetry should be recorded.

- Clinical assessment of a breathless patient starts with "ABC" (airway, breathing, circulation) (see recommendation 7).

- A brief history should be taken from the patient or other informant.

- Initial assessment should include pulse and respiratory rate in all cases (see recommendation 7).

- Pulse oximetry should always be measured in patients with breathlessness or suspected hypoxaemia (see recommendation 9).

- Disease-specific measurements should also be recorded (eg, peak expiratory flow in asthma, blood pressure in cardiac disease).

\subsection{Immediate management of hypoxaemic patients}

Having ascertained that the airway is clear, the emergency responders should commence oxygen treatment if the oxygen saturation is below the target. The initial oxygen therapy should follow the general principles given in tables $1-4$ and charts 1 and 2 (figs 1 and 2). There is some evidence that bronchodilator therapy, however given, can cause increased V/ $\mathrm{Q}$ mismatch and reduced blood oxygen levels in acutely ill patients shortly after treatment (see section 10.8.1).

\section{Recommendations}

- The initial oxygen therapy to be used in the various clinical situations is given in tables 1-4.

- If there is a clear history of asthma or heart failure or other treatable illness, appropriate treatment should be instituted in accordance with guidelines or standard management plans for each disease. [Grade D]

21. The oxygen saturation should be monitored continuously until the patient is stable or arrives at hospital for a full assessment. The oxygen concen- 
tration should be adjusted upwards or downwards to maintain the target saturation range. [Grade $D$ ]

22. In most emergency situations oxygen is given to patients immediately without a formal prescription or drug order. The lack of a prescription should never preclude oxygen being given when needed in an emergency situation. However, a subsequent written record must be made of what oxygen therapy has been given to every patient (in a similar manner to the recording of all other emergency treatment). [Grade D]

\subsection{Patients with known COPD}

A proportion of breathless patients will have COPD (chronic bronchitis and emphysema). Unfortunately, a recent Cochrane review of oxygen therapy for COPD in the prehospital setting found no relevant studies. ${ }^{282}$

Audits of emergency admissions in UK hospitals have shown that about $25 \%$ of breathless medical patients who require hospital admission have COPD as a main diagnosis. Many of these patients will require carefully controlled oxygen therapy because they are at risk of carbon dioxide retention or respiratory acidosis. In a large UK study, ${ }^{34} 47 \%$ of patients with exacerbated COPD had $\mathrm{PaCO}_{2}>6.0 \mathrm{kPa}$ (45 mm Hg), $20 \%$ had respiratory acidosis $\left(\mathrm{pH}<7.35\right.$ or $\left.\left[\mathrm{H}^{+}\right]>45 \mathrm{nmol} / \mathrm{l}\right)$ and $4.6 \%$ had severe acidosis ( $\mathrm{pH}<7.25$ or $\left[\mathrm{H}^{+}\right]>56 \mathrm{nmol} / \mathrm{l}$ ). Acidosis was more common if the blood oxygen was $>10 \mathrm{kPa}$ (75 mm Hg). Plant and colleagues ${ }^{34}$ recommended that patients with acute $\mathrm{COPD}$ should be maintained within a $\mathrm{PaO}_{2}$ range of $7.3-10 \mathrm{kPa}(55-75 \mathrm{~mm} \mathrm{Hg})$ to avoid the dangers of hypoxaemia and acidosis.

\section{Recommendation (see table 3)}

- Patients with COPD should initially be given oxygen via a Venturi $28 \%$ mask at a flow rate of $4 \mathrm{l} / \mathrm{min}$ or a $24 \%$ Venturi mask at a flow rate of $2 \mathrm{l} / \mathrm{min}$. Some patients may benefit from higher flow rates via the Venturi mask (see recommendation 32). The target oxygen saturation should be 88$92 \%$ in most cases or an individualised saturation range based on the patient's blood gas measurements during previous exacerbations. [Grade C]

\subsection{Patients who should be assumed to have COPD}

One of the challenges faced by the initial clinical response staff is that the diagnosis may be unclear and the patient's medical records or detailed history may not be available. It has been shown that ambulance teams may be aware of a diagnosis of COPD in only $58 \%$ of cases. ${ }^{283}$

The guidelines group consider that an initial diagnosis of COPD should be assumed if there is no clear history of asthma and the patient is $>50$ years of age and a long-term smoker or ex-smoker with a history of longstanding breathlessness on minor exertion. The diagnosis should be reassessed on arrival at hospital where more information will probably become available, and the $\mathrm{FEV}_{1}$ should be measured unless the patient is too breathless to undertake spirometry.

\section{Recommendation}

- If the diagnosis is unknown, patients $>50$ years of age who are long-term smokers with a history of chronic breathlessness on minor exertion such as walking on level ground and no other known cause of breathlessness should be treated as if having COPD for the purposes of this guideline.
Patients with COPD may also use terms such as chronic bronchitis and emphysema to describe their condition but may sometimes mistakenly use "asthma" (see table 3).

9.6 Other patients at risk of hypercapnic respiratory failure with respiratory acidosis

- Any patient with severe kyphoscoliosis or severe ankylosing spondylitis.

- Severe lung scarring from old tuberculosis (especially with thoracoplasty).

- Morbid obesity (body mass index $>40 \mathrm{~kg} / \mathrm{m}^{2}$ ).

- Patients with neuromuscular disorders (especially if muscle weakness has led to wheelchair use).

- Any patient on home mechanical ventilation.

- Use of home mechanical ventilation.

- Overdose of opiates, benzodiazepines or other drugs causing respiratory depression.

\subsection{0xygen alert cards and $24 \%$ or $28 \%$ Venturi masks in patients with COPD (and others at risk of respiratory acidosis) who have had an episode of hypercapnic respiratory failure}

The administration of high oxygen concentrations in acute COPD and other conditions (see section 8.12) leads to worsening of hypercapnic respiratory failure and respiratory acidosis. ${ }^{34}$ Patients with COPD and a $\mathrm{PaO}_{2}>10 \mathrm{kPa}(75 \mathrm{~mm} \mathrm{Hg})$ and a $\mathrm{PaCO}_{2}$ $>6.0 \mathrm{kPa}$ (45 $\mathrm{mm} \mathrm{Hg}$ ) may be assumed to have had excessive oxygen therapy. If a patient is found to have respiratory acidosis due to excessive oxygen therapy, the oxygen therapy should not be discontinued immediately because the oxygen level will fall significantly over 1-2 min by virtue of the alveolar gas equation (see section 5.2.1) whereas the carbon dioxide level will take much longer to correct itself (see section 6.3.2). In this situation the oxygen treatment should be stepped down to $28 \%$ or $24 \%$ oxygen from a Venturi mask depending on oxygen saturation and blood gas results. A saturation target of $88-92 \%$ is recommended for acidotic patients in type 2 respiratory failure and non-invasive ventilation is required if the acidosis does not resolve quickly. ${ }^{25} 34$ This avoidable problem has occurred historically during the transfer to hospital, prior to measurement of arterial blood gases or before a definitive diagnosis is known. Furthermore, ambulance teams are often not informed at present of a diagnosis of COPD ${ }^{283}$ and may not be aware of the presence of other high-risk conditions such as kyphoscoliosis or respiratory failure due to neuromuscular conditions. These patients can be issued with an oxygen alert card and a $24 \%$ or $28 \%$ Venturi mask based on previous blood gas results. The recommended oxygen saturation will be based on the clinical scenario for each individual patient but will usually be $88-92 \%$, occasionally $85-88 \%$ or $85-90 \%$ based on previous blood gas results. Patients should be instructed to show this card to the ambulance crew and emergency department staff in order to avoid the use of high oxygen concentrations. This scheme can be successful. ${ }^{284}$ The ambulance service can also be informed about which patients are issued with oxygen alert cards. ${ }^{285}$ The current Joint Royal Colleges Ambulance Liaison Committee (JRCALC) guideline for the use of oxygen in COPD are being revised to accommodate these changes. ${ }^{274}$ An example of an oxygen alert card is shown in fig 8 .

\section{Recommendations}

23. Patients with COPD (and other at-risk conditions) who have had an episode of hypercapnic respiratory failure should be issued with an oxygen alert card 
Oxygen alert card

Name:

I am at risk of type II respiratory failure with a raised $\mathrm{CO}_{2}$ level.

Please use my $\quad \%$ Venturi mask to achieve an

oxygen saturation of ___ $\%$ to __ $\%$ during exacerbations

Use compressed air to drive nebulisers (with nasal oxygen at $2 \mathrm{l} / \mathrm{min}$ )

If compressed air not available, limit oxygen-driven nebulisers to 6 minutes.

Figure 8 Example of oxygen alert card.

and with a $24 \%$ or $28 \%$ Venturi mask. They should be instructed to show the card to the ambulance crew and emergency department staff in the event of an exacerbation. [Grade C]

24. The content of the alert card should be specified by the physician in charge of the patient's care, based on previous blood gas results. [Grade D]

25. The primary care team and ambulance service should also be informed by the responsible clinician that the patient has had an episode of hypercapnic respiratory failure and carries an oxygen alert card. The home address and ideal oxygen dose or target saturation ranges of these patients can be flagged in the ambulance control systems and disseminated to ambulance crews when required. [Grade D]

26. Out-of-hours services providing emergency primary care services should be informed by a responsible clinician that the patient has had an episode of hypercapnic respiratory failure and carries an oxygen alert card. Use of oxygen in these patients will be guided by the instructions on the alert card. [Grade D]

27. During ambulance journeys oxygen-driven nebulisers should be used for patients with asthma and may be used for patients with COPD in the absence of an air-driven compressor system. If oxygen is used for patients with known COPD, its use should be limited to $6 \mathrm{~min}$. This will deliver most of the nebulised drug dose but limit the risk of hypercapnic respiratory failure (section 10.8.2). [Grade D]

28. If a patient is suspected to have hypercapnia or respiratory acidosis due to excessive oxygen therapy, the oxygen therapy should not be discontinued but should be stepped down to $28 \%$ or $24 \%$ oxygen from a Venturi mask depending on oxygen saturation and subsequent blood gas results. [Grade $\mathrm{C}$ ]

\subsection{Choice of devices in prehospital care}

The range of oxygen delivery devices is very wide as discussed in section 10. However, most patients can be managed with one of five types of oxygen delivery device.
Recommendation

29a. It is recommended that the following delivery devices should be available in prehospital settings where oxygen is administered (see section 10): [Grade D]

- high concentration reservoir mask (non-rebreathe mask) for high-dose oxygen therapy;

- nasal cannulae (preferably) or a simple face mask for medium-dose oxygen therapy;

- $28 \%$ Venturi mask for patients with definite or likely COPD (patients who have an oxygen alert card may have their own $24 \%$ or $28 \%$ Venturi mask);

- tracheostomy masks for patients with tracheostomy or previous laryngectomy.

\section{SECTION 10: PRACTICAL ASPECTS OF OXYGEN THERAPY}

Oxygen delivery systems can be considered as two components:

- the method of storage and provision of oxygen (eg, cylinders);

- the method of delivery to the patient (eg, Venturi mask).

The options available for both will depend on the environment in which it is being used and the needs of the patient.

\subsection{0xygen storage and provision}

10.1.1 Cylinders (compressed gas)

Cylinders contain compressed gas held under a very high pressure. They come in an array of sizes and hence capacity, ranging from small portable cylinders for individual patient use to large cylinders suitable for hospital use (table 10). These can be used for bedside administration where piped oxygen is not available or can be the supply for a piped system.

With recent changes in technology, high pressure cylinders are now available (ie, filled to 200 bar rather than 137 bar which can contain $54 \%$ more gas for the same size cylinder). It is important for all users of oxygen to be aware that most oxygen cylinders are colour-coded (black cylinder with white shoulder). Small lightweight cylinders are also available for ambulatory use (eg, some weigh $3.2 \mathrm{~kg}$ when full). All systems containing compressed gases in the UK are subject to the Pressure Systems Safety Regulations 2000 (SI 2000 No 128). These regulations are intended to prevent the risk of injury from pressurised systems.

- Trusts must ensure that they have a policy in place which ensures the safety of patients, staff and contractors in the provision, storage, use and maintenance of compressed gas systems as required by the Health and Safety at Work etc Act 1974.

- Clinicians using oxygen cylinders should check the labelling of the cylinder to ensure that it is an oxygen cylinder and checks should be made to ensure that the cylinder is not empty or near-empty.

\subsubsection{Liquid oxygen}

Liquid oxygen is contained in pressure tanks and is obtained from atmospheric oxygen by fractional distillation. It has to be evaporated into a gas before use. Large tanks are often used by

Table 10 Examples of common oxygen cylinder sizes and capacities

\begin{tabular}{|c|c|c|c|c|c|c|c|c|}
\hline \multicolumn{9}{|c|}{ Oxygen cylinder sizes } \\
\hline Size & $\mathrm{C}$ & $\mathrm{CD}$ & $\mathrm{D}$ & $E$ & $\mathrm{~F}$ & $G$ & $H X$ & $\mathrm{~J}$ \\
\hline Height $(\mathrm{cm})$ & 36 & 49 & 46 & 79 & 86 & 124 & 94 & 145 \\
\hline Capacity (I) & 170 & 460 & 340 & 680 & 1360 & 3400 & 2300 & 6800 \\
\hline
\end{tabular}


hospitals and small tanks can be used domestically. Portable liquid oxygen is also available in small portable containers which can be filled from the larger tanks.

\subsubsection{0xygen concentrators}

Oxygen concentrators are largely used in the domiciliary setting for the provision of long-term oxygen therapy and are therefore not used in the acute setting so will not be covered further.

\subsection{Patient delivery methods/interfaces}

10.2.1 High concentration reservoir mask (non-rebreathing mask) (fig 9)

This type of mask delivers oxygen at concentrations between $60 \%$ and $90 \%$ when used at a flow rate of $10-151 / \mathrm{min}^{286}$ The concentration is not accurate and will depend on the flow of oxygen and the patient's breathing pattern. These masks are most suitable for trauma and emergency use where carbon dioxide retention is unlikely (table 1 ).

\subsubsection{Simple face mask (fig 10)}

This type of mask delivers oxygen concentrations between $40 \%$ and $60 \%$. It is sometimes referred to as an MC Mask, Medium Concentration Mask, Mary Catterall Mask or as a "Hudson Mask", but the latter description is discouraged because the Hudson Company make many types of mask (including high concentration reservoir masks). The guideline group favours the term "simple face mask". The oxygen supplied to the patient will be of variable concentration depending on the flow of oxygen and the patient's breathing pattern. The concentration can be changed by increasing or decreasing the oxygen flows between 5 and $10 \mathrm{l} / \mathrm{min}$. However, different brands of simple face mask can deliver a different oxygen concentration at a given flow rate. Flows of $<51 / \mathrm{min}$ can cause increased resistance to breathing, and there is a possibility of a build-up of carbon dioxide within the mask and rebreathing may occur. ${ }^{287}$

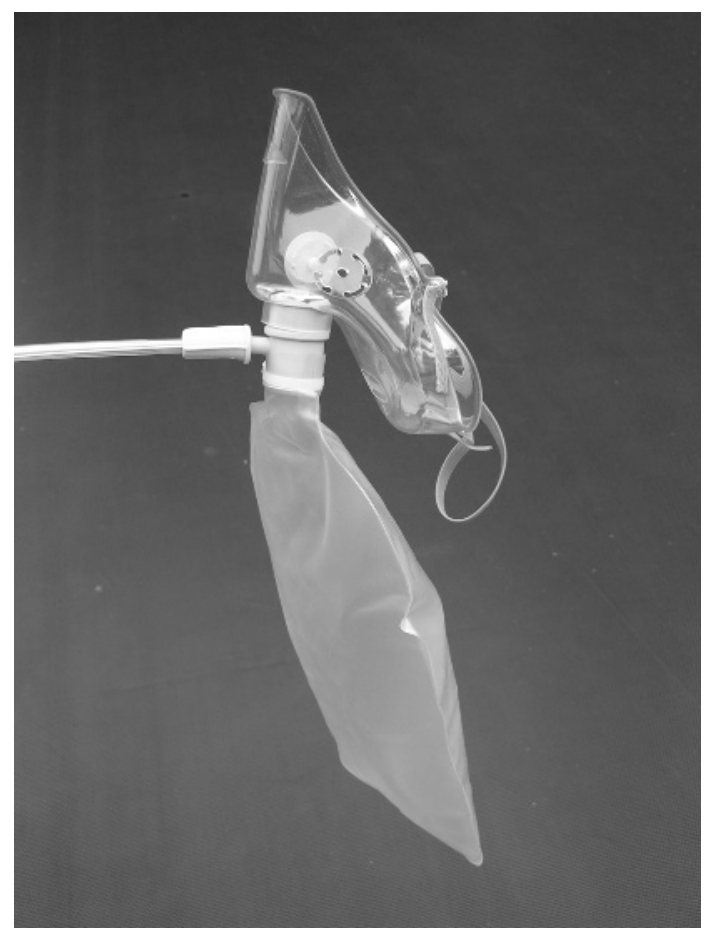

Figure 9 High concentration reservoir mask (non-rebreathing mask).

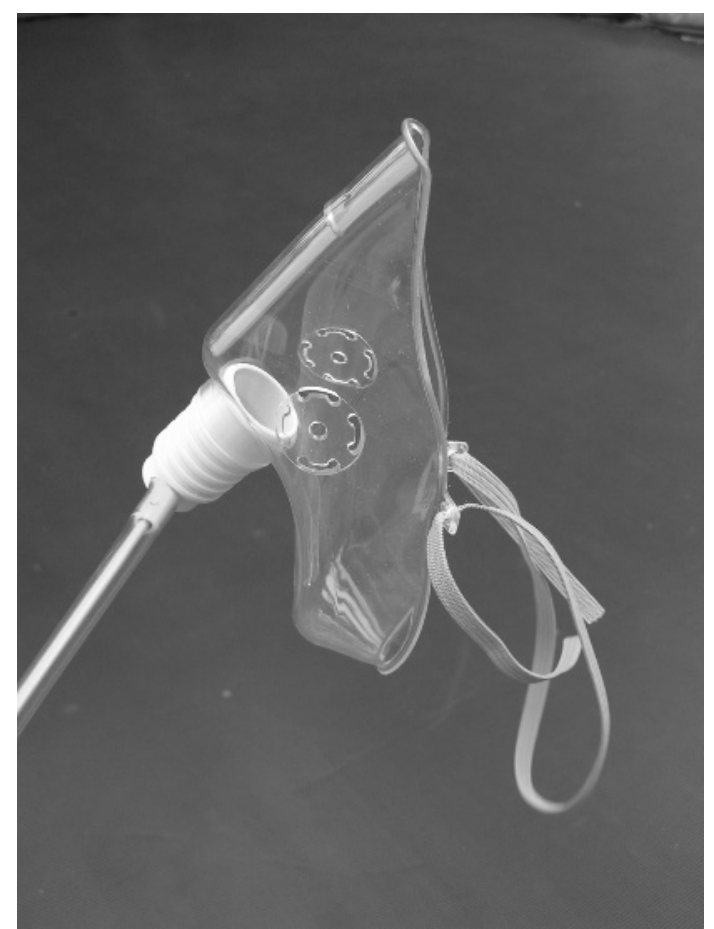

Figure 10 Simple face mask.

This mask is suitable for patients with hypoxaemic respiratory failure (type 1) but is not suitable for patients with hypercapnic (type 2) respiratory failure. The mask may deliver a high concentration of oxygen $(>50 \%)$ and is therefore not recommended for patients who require low-dose oxygen therapy because of the risk of carbon dioxide retention. Patients using a simple face mask may have an inspiratory flow rate greater than the gas flow rate from the mask, so the simple face mask should not be used at flow rates below $5 \mathrm{l} / \mathrm{min}{ }^{287}$ Several publications have shown that patients who require medium-dose oxygen therapy tend to prefer nasal cannulae to simple face masks and the cannulae are more likely to be left in position by the patient and less likely to fall off. ${ }^{288-291}$

\subsubsection{Venturi mask (fig 11)}

A Venturi mask will give an accurate concentration of oxygen to the patient regardless of oxygen flow rate (the minimum suggested flow rate is written on each Venturi device and the available options are shown in table 11). The oxygen concentration remains constant because of the Venturi principle. The gas flow into the mask is diluted with air which is entrained via the cage on the Venturi adaptor. The amount of air sucked into the cage is related to the flow of oxygen into the Venturi system. The higher the flow the more air is sucked in. The proportions remain the same and therefore the Venturi mask delivers the same concentration of oxygen as the flow rate is increased.

Venturi masks are available in the following concentrations: $24 \%, 28 \%, 35 \%, 40 \%$ and $60 \%$. They are suitable for all patients needing a known concentration of oxygen, but $24 \%$ and $28 \%$ Venturi masks are particularly suited to those at risk of carbon dioxide retention (eg, patients with COPD). A further benefit of Venturi masks is that the flow rate of gas from the mask will usually exceed the inspiratory flow rate of the patient. One study has shown that patients with a respiratory rate $>30$ breaths/min often have an inspiratory flow rate above 

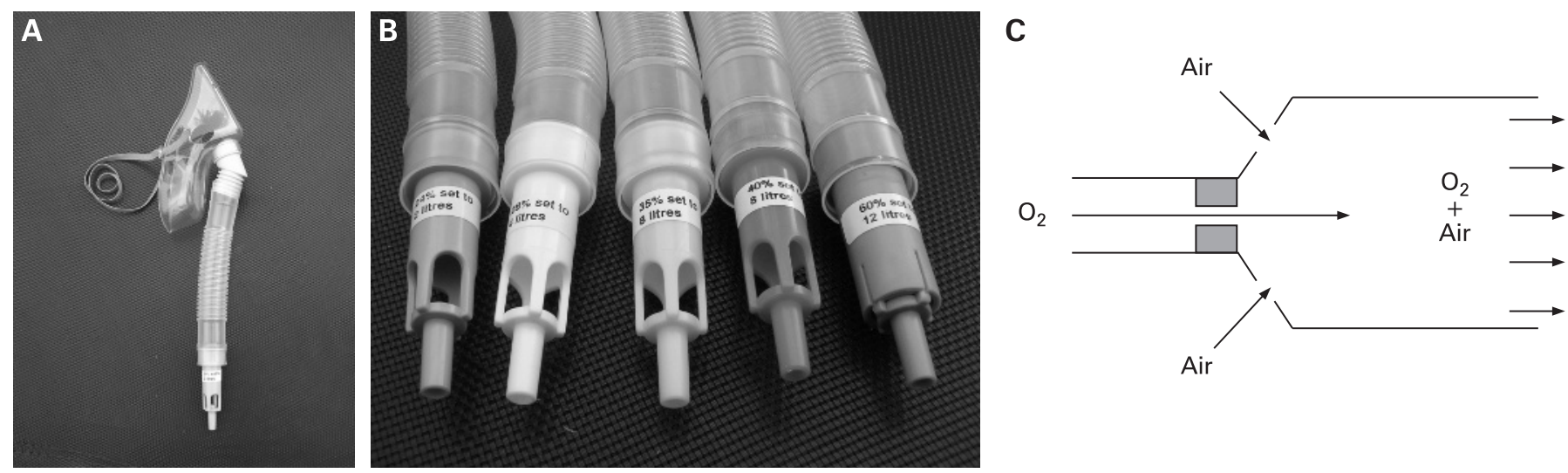

Figure 11 (A) Venturi mask. (B) Range of concentrations available. (C) Operation of Venturi valve. For 24\% Venturi mask the typical oxygen flow of 2 $\mathrm{l} / \mathrm{min}$ gives a total gas flow of $51 \mathrm{l} / \mathrm{min}$. For $28 \%$ Venturi mask, $4 \mathrm{l} / \mathrm{min}$ oxygen flow gives a total gas flow of $44 \mathrm{l} / \mathrm{min}$ (table 11 ).

the minimum flow rate specified on the mask packaging. ${ }^{254}$ Therefore, for patients with a high respiratory rate, it is suggested that the flow rate for Venturi masks should be set above the minimum flow rate listed on the packaging (increasing the oxygen flow rate into a Venturi mask does not increase the concentration of oxygen which is delivered). The accuracy of oxygen delivery from a Venturi mask is greatly reduced if the mask is not accurately placed on the patient's face. $^{292}$

- Patients with a respiratory rate $>30$ breaths/min often have a flow rate which is above the minimum delivered by the Venturi system as specified by the flow rate recommended for the mask. [Evidence III]

Venturi masks deliver a constant percentage of oxygen but the effect on the patient will depend on the condition being treated and on the breathing pattern and baseline oxygen saturation of the patient. As might be expected from the oxygen dissociation curve, patients with an oxygen saturation that is already in the normal range will have a very small rise in oxygen saturation (although the arterial oxygen tension is likely to rise substantially). However, patients with very low oxygen saturation will have a marked rise if given even a small dose of oxygen. This is because the oxygen dissociation curve is actually a "rapid escalator" rather than a "slippery slope". 9397 This is illustrated in fig 12 which uses actual oxygen saturations from King et $a l^{246}$ and Warrel et al $l^{65}$ together with calculated saturations from Bone et a ${ }^{293}$ (two different groups), DeGaute et $a l^{294}$ and Schiff and Massaro. ${ }^{245}$

\subsubsection{Nasal cannulae (fig 13)}

Nasal cannulae can be used to deliver low- and medium-dose oxygen concentrations. However, there is wide variation in patients' breathing patterns so the same flow rate of nasal oxygen may have widely different effects on the blood oxygen and carbon dioxide levels of different patients. Nasal cannulae at $1-4 \mathrm{l} / \mathrm{min}$ can have effects on oxygen saturation approximately equivalent to those seen with $24-40 \%$ oxygen from Venturi masks. ${ }^{188} 295$ The oxygen dose continues to rise up to flows above $6 \mathrm{l} / \mathrm{min},{ }^{188} 296$ but some patients may experience discomfort and nasal dryness at flows above $4 \mathrm{l} / \mathrm{min}$, especially if maintained for several hours. Although one might expect mouth breathing to reduce the efficiency of nasal cannulae, the majority of studies have shown that mouth breathing results in either the same inspired oxygen concentraton or a higher concentration, especially when the respiratory rate is increased. ${ }^{296}$ This is important because patients with acute breathlessness are likely to breathe quickly and via the mouth rather than the nose. As there is marked individual variation in breathing pattern, the flow rate must be adjusted based on oximetry measurements and, where necessary, blood gas measurements. A crossover comparison of nasal cannulae versus a Venturi mask (both adjusted to give satisfactory initial oxygen saturation) showed that the oxygen saturation of patients with exacerbated COPD fell below $90 \%$ for $5.4 \mathrm{~h} /$ day during treatment with a Venturi mask compared with only $3.7 \mathrm{~h}$ /day during treatment with nasal cannulae. ${ }^{297}$

The upper range of oxygen delivery from nasal cannulae is a little lower than the output of a simple face mask, but the lower range goes a lot lower than a simple face mask which should not be used below a flow rate of $5 \mathrm{l} / \mathrm{min}$ (about $40 \%$ oxygen). ${ }^{287}$ The performance and variation of nasal cannulae for medium concentration oxygen therapy is broadly similar to that of the simple face mask, both in laboratory experiments ${ }^{188} 296$ and in clinical practice. ${ }^{289-291298} 299$ One study ${ }^{299}$ suggested that the saturation was lower with nasal cannulae than with simple face masks in a subgroup of men following abdominal surgery. Further studies are required to see if this was a chance finding or

Table 11 Total gas flow rate ( $/ / \mathrm{min})$ from Venturi masks at different oxygen flow rates

\begin{tabular}{|c|c|c|c|c|c|}
\hline \multirow[b]{2}{*}{ Oxygen flow (l/min) } & \multicolumn{5}{|c|}{ Venturi values } \\
\hline & $24 \%$ oxygen & $28 \%$ oxygen & $35 \%$ oxygen & $40 \%$ oxygen & 60\% oxygen \\
\hline 15 & & & 84 & 82 & 30 \\
\hline 12 & & & 67 & 50 & 24 \\
\hline 10 & & & 56 & 41 & \\
\hline 8 & & 89 & 46 & & \\
\hline 6 & & 67 & & & \\
\hline 4 & 102 & 44 & & & \\
\hline 2 & 51 & & & & \\
\hline
\end{tabular}


Figure 12 0xygen saturation response to treatment with $24 \%, 28 \%$ and $35 \%$ oxygen in patients with COPD.
Oxygen saturation on air and controlled oxygen therapy

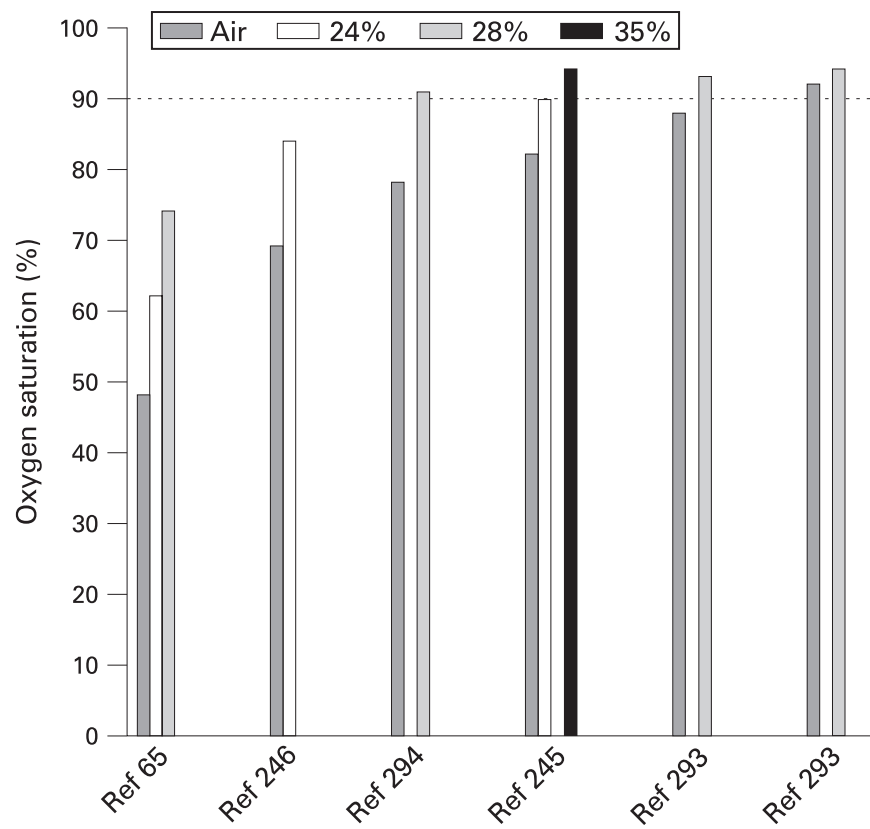

a genuine clinical difference between the devices. Three patient preference studies comparing nasal cannulae with simple face masks in postoperative care found that patient preference was strongly in favour of nasal cannulae with up to $88 \%$ of patients preferring cannulae to masks. ${ }^{289-291}$ Another advantage of cannulae over simple face masks is that they are less likely to be removed accidentally and they allow the patient to speak and eat. ${ }^{290} 300$ There are no comparisons of these devices in acute care, but there is no reason to believe that the results would be any different for patients requiring medium-dose oxygen therapy.

Advantages of nasal cannulae compared with simple face masks for medium-dose oxygen therapy (Evidence level III):

- Comfort (but a minority of patients dislike the flow of oxygen into the nose, especially above $4 \mathrm{l} / \mathrm{min}$ ).

- Adjustable flow gives wide oxygen dose range (flow rate of $1-6 \mathrm{l} / \mathrm{min}$ gives $\mathrm{FIO}_{2}$ from approximately $24 \%$ to approximately 50\%).

- Patient preference.

- No claustrophobic sensation.

Figure 13 Nasal cannulae.
- Not taken off to eat or speak and less likely to fall off.

- Less affected by movement of face.

- Less inspiratory resistance than simple face masks.

- No risk of rebreathing of carbon dioxide.

- Cheaper. Disadvantages of nasal cannulae:

- May cause nasal irritation or soreness.

- Will not work if nose is severely congested or blocked.

\subsubsection{Tracheostomy mask (fig 14)}

These devices are designed to allow oxygen to be given via a tracheostomy tube or to patients with previous laryngectomy (ie, "neck breathing patients"). The oxygen flow rate should be adjusted to achieve saturation in accordance with tables 1-4 and chart 1 (fig 1). Oxygen given in this way for prolonged periods needs constant humidification and patients may need suction to remove mucus from the airway.
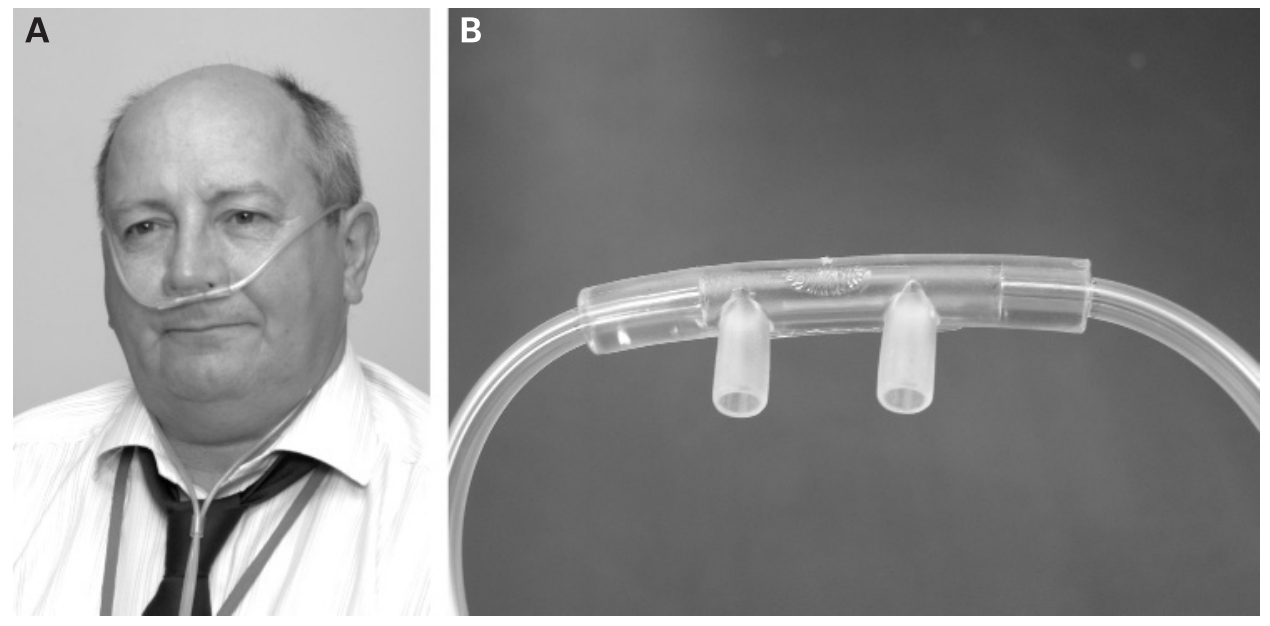


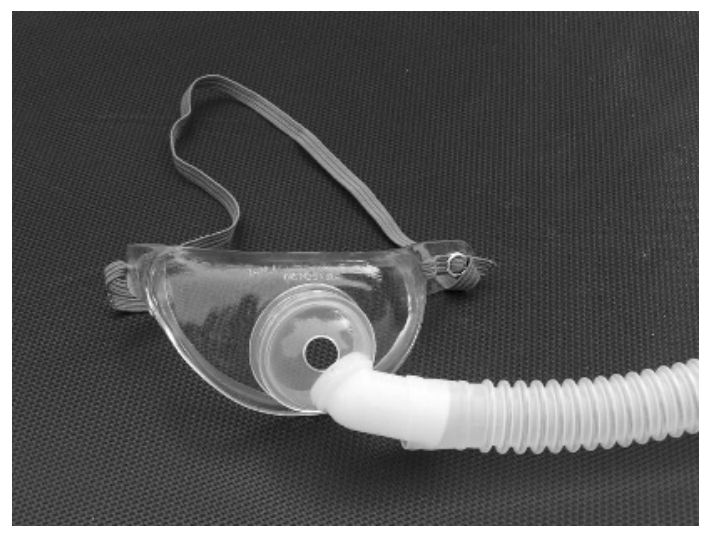

Figure 14 Tracheostomy mask.

\subsubsection{Continuous positive airways pressure and non-invasive ventilation}

These treatment options are beyond the scope of the present guideline. Readers are referred to the BTS guideline concerning the use of non-invasive ventilation in patients with exacerbations of COPD. ${ }^{32}$

\subsection{0xygen carriage and delivery during patient transport in ambulances}

Transport of oxygen cylinders in vehicles comes under the Transport of Dangerous Substances Act or the Carriage Regulations only if 1000 litres or more (measured by the water capacity of the cylinder) is carried at any one time. Ambulances are therefore exempt from this. Normal health and safety requirements will still apply. ${ }^{301} 302$

\subsubsection{Health and Safety Executive guidance for safe use of oxygen} cylinders $^{301} 302$

- All cylinders must be secured appropriately so they cannot move in transit (includes portable cylinders).

- No smoking in the vicinity of cylinders.

- Cylinders must be checked regularly for obvious signs of leakage.

- Cylinders must be kept out of direct sunlight.

- Green warning triangle "compressed gas" should be displayed on the vehicle.

- Cylinders should never be lifted by the neck.

- They should only be changed by suitably trained personnel.

- Apart from portable cylinders, all cylinders should be moved using a cylinder trolley.

\subsubsection{0xygen use by UK ambulance services}

Currently within the UK the Ambulance Service-whether NHS or private-has a range of vehicles and oxygen delivery systems at their disposal. There is an increasing use of cycle response units which tend to use the lightweight $A Z$ or $C$ sized cylinder with a capacity of 170 litres. Motorcycle response units are generally equipped with the same AZ or C sized cylinders. Fast response units based on cars tend to be equipped with at least two of the lightweight CD sized cylinders which hold 460 litres. The CD cylinder is also the size favoured by mountain, cave and mines rescue teams.

Front-line ambulances are usually equipped with piped oxygen fittings (Schraeder type) and supplied from two HX sized (2300 litres) cylinders, as well as carrying at least two CD sized cylinders to power a portable oxygen-powered resuscitator. The piped supply has several outlet points placed in strategic positions to which are attached standard Schrader flow meters $(0-15 \mathrm{l} / \mathrm{min})$. This enables oxygen to be given throughout the patient's journey. The ambulance is also equipped with a portable supply which can be used at the site of an accident, taken into a patient's home or can be used when transferring a patient. They carry a range of patient interfaces for delivering the oxygen under the different circumstances encountered.

Portable resuscitators are always capable of supplying freeflow oxygen therapy as well as their resuscitator facility. Again, there are a variety of portable oxygen-powered resuscitators and it is beyond the scope of these guidelines to describe each one available for use in prehospital care. It is strongly suggested that those practitioners who need to work closely with the Ambulance Service should become familiar with the equipment used by their local Ambulance Service provider. With the possible exception of the cycle response units, all types of Ambulance Service response will have portable resuscitators, bag-valve-mask devices and portable suction as a minimum. Front-line emergency department ambulances will also have vehicle-powered suction available.

It is also very common now for patient transport service ambulances to be equipped with an oxygen supply, normally an HX cylinder (2300 litres) delivering the oxygen via a flow meter attached directly to the cylinder. Such vehicles also tend to carry basic hand-held suction devices. The masks available are generally high concentration reservoir masks and are provided specifically for emergency use for patients who might become ill on the vehicle. Vehicles that are equipped with an oxygen supply should also carry oximeters to ensure appropriate use of oxygen (see section 9.8 for advice on which oxygen delivery devices should be carried in ambulances).

\subsection{0xygen carriage in other vehicles and in primary care settings and patients' homes}

10.4.1 0xygen carriage in private cars (Health and Safety Executive ${\text { Guidance })^{301} 302}$

When travelling by car, patients have the freedom to carry their own portable oxygen cylinder. Some general practitioners in rural areas also carry oxygen in their cars. However, it is advised that certain safety precautions should be followed:

- It is good practice for the car to display a green warning triangle for "compressed gas".

- The cylinder should be secure within the car and cannot move during transport or in the event of an accident.

\subsubsection{Medical centres and primary care practices}

The majority of medical centres and practices should have a supply of oxygen for emergency use. Generally, cylinders with integral high-flow regulators should be ordered. Otherwise, the cylinder must be fitted with a high-flow regulator capable of delivering a flow of $>6 \mathrm{l} / \mathrm{min}$ in order to deliver medium and high-dose oxygen therapy. A recommended list of oxygen delivery devices for use in prehospital care is given in section 9.8.

- Emergency oxygen should be available in primary care medical centres, preferably using oxygen cylinders with integral high-flow regulators. Alternatively, oxygen cylinders fitted with high-flow regulators (delivering $>6 \mathrm{l} / \mathrm{min}$ ) must be used (see recommendation 19). 


\subsubsection{Patients' homes}

In patients' homes oxygen is either provided for long-term therapy where an oxygen concentrator is provided (with or without a lightweight cylinder for ambulatory needs) or for short-term/short-burst therapy. Long-term oxygen therapy is covered in other guidelines. This existing home oxygen supply may be used by a patient or general practitioner in an emergency situation before the arrival of an ambulance.

The patient/carers should be made aware of the following Health and Safety recommendations: ${ }^{301}$

- All cylinders should be stored on a cylinder trolley or suitably secured so they cannot be knocked over.

- There should be no trailing oxygen tubing.

- A green warning triangle for "compressed gas" should be displayed by the front door (warns emergency services in the event of a fire).

- The minimum number of cylinders should be stored in the house.

- There should be no smoking in the vicinity of oxygen cylinders.

- Cylinders must be checked regularly for obvious signs of leakage.

- Cylinders must be kept out of direct sunlight.

- Oxygen must not be used near a naked flame.

\subsection{Oxygen delivery systems in hospitals}

Most hospitals have piped oxygen systems as described previously, although some wards can still be found where piped oxygen is not available and large compressed gas cylinders are used to supply the oxygen. Acute hospitals can spend up to $£ 100000$ per annum on liquid oxygen, so any device that uses lower oxygen flow rates could have significant economic savings for hospitals (eg, nasal cannulae instead of a simple face mask for medium-dose oxygen).

\subsubsection{Postoperative care on general surgical wards}

Medium concentration masks and nasal cannulae are usually sufficient (target saturation 94-98\%) except for patients with known significant COPD who should receive oxygen from a $24 \%$ or $28 \%$ Venturi mask or $1-21 / \mathrm{min}$ from nasal cannulae aiming at a saturation range of $88-92 \%$.

\subsubsection{Emergency departments}

Medium or high concentration oxygen is normally used (via nasal cannulae, simple face mask or reservoir mask), but particular attention should be given to patients who have type 2 respiratory failure when a $24 \%$ or $28 \%$ Venturi mask or nasal cannulae at a flow rate of $1-21 / \mathrm{min}$ would be appropriate.

\subsubsection{General wards and respiratory wards}

The method of oxygen delivery will depend on the following circumstances:

- Expected duration of treatment.

- Type of respiratory illness.

- Pattern of breathing (high or low respiratory rate and drive).

- Need for humidification.

- Risk of carbon dioxide retention.

- Presence of confusion and its effect on potential compliance. Nasal cannulae, simple face masks, reservoir masks and Venturi masks should be used where appropriate (see tables 1-4 and charts 1 and 2 (figs 1 and 2)). Nasal cannulae at flow rates or $0.5-1.0 \mathrm{l} / \mathrm{min}$ are sometimes used as a substitute for Venturi masks in acute or post-acute patients with COPD on respiratory wards (adjusting flow as necessary to achieve the desired arterial blood gas tensions). This practice requires the use of paediatric flow meters to ensure consistent and finely calibrated oxygen delivery and is not recommended outside specialist units.

\subsubsection{Devices used in emergency oxygen therapy}

Based on the advantages of each delivery system discussed above, the following recommendations are made for delivery of oxygen in medical emergencies. It is likely that additional equipment will be maintained in specialist units, but specialist treatment is outside the scope of the present guideline.

\section{Recommendations (see tables 1-4)}

29b. Most hospital patients can be managed with the same delivery device as in recommendation 29a, but a $24 \%$ Venturi mask should also be available.

- The high concentration reservoir mask at $10-15 \mathrm{l} / \mathrm{min}$ is the preferred means for delivering high-dose oxygen to critically ill patients. [Grade D]

- Nasal cannulae should be used rather than simple face masks in most situations requiring medium-dose oxygen therapy. Nasal cannulae are preferred by patients for reasons of comfort and they are less likely to be removed during meals (see section 10.2.4). There is also a cost saving. [Grade $\mathbf{C}$ ]

- The flow rate from nasal cannulae for medium-dose oxygen therapy should be adjusted between 2 and $6 \mathrm{l} /$ min to achieve the desired target saturation. [Grade $\mathbf{C}$ ]

- Venturi masks are recommended for patients requiring precise control of $\mathrm{FIO}_{2}$. Venturi masks can deliver a constant $\mathrm{FIO}_{2}$ of $24 \%, 28 \%, 35 \%, 40 \%$ and $60 \%$ oxygen with a greater gas flow than a simple face mask. In those at risk of developing hypercapnic respiratory failure with oxygen therapy, the use of Venturi masks may reduce this risk. Furthermore, there is less likelihood of dilution of the oxygen stream by room air if the patient's inspiratory flow rate exceeds the flow rate delivered by the face mask. [Grade D]

30. For many patients Venturi masks can be substituted with nasal cannulae at low flow rates (1-2 $1 / \mathrm{min})$ to achieve the same target range once patients have stabilised. [Grade D]

31. The flow rate from simple face masks should be adjusted between 5 and $10 \mathrm{l} / \mathrm{min}$ to achieve the desired target saturation. Flow rates below $5 \mathrm{l} / \mathrm{min}$ may cause carbon dioxide rebreathing and increased resistance to inspiration. [Grade $\mathrm{C}$ ]

32. Patients with COPD with a respiratory rate of $>\mathbf{3 0}$ breaths/min should have the flow rate set to $50 \%$ above the minimum flow rate specified for the Venturi mask and/or packaging (increasing the oxygen flow rate into a Venturi mask increases the total gas flow from the mask but does not increase the concentration of oxygen which is delivered). [Grade $\mathrm{C}$ ]

\subsubsection{Flow meters}

All oxygen delivery systems must have a method of taking the high pressure/flow of gas and reducing it so it can be administered to the patient at a specific flow depending on the individual's needs and mask being used. 
Piped oxygen points have Schrader flow meters and cylinders have pressure and flow regulators. Most oxygen flow meters use a floating ball to indicate the flow rate. The centre of the ball should be aligned with the appropriate flow rate marking. The example shown in fig 15 indicates the correct setting to deliver $2 \mathrm{l} / \mathrm{min}$.

\subsubsection{Oxygen tubing and oxygen wall outlets}

Oxygen tubing is needed to connect flow meters and regulators to the patient delivery device. It is important to ensure that all tubing is connected correctly at both ends. The National Patient Safety Agency has reported frequent adverse events related to oxygen use, including four reports of instances where an oxygen mask was connected in error to a compressed air outlet instead of an oxygen outlet. Compressed air outlets are often used to drive nebulisers on in hospitals because they are quieter than electrical compressors. However, the flow meter looks very similar to an oxygen flow meter and is often mounted beside an oxygen flow meter so it is very important to ensure that air flow meters are clearly labelled. There is a similar risk with other piped gas outlets such as those delivering nitrous oxide in some hospitals. Air flow meters are never required in an emergency and should be removed from wall sockets or covered by a designated "hood" when not in use. The guideline authors are also aware of some cases where twin oxygen outlets were in use and the wrong one had been turned on or off. For example, one patient tried to turn off the oxygen flow after finishing a nebulised treatment but accidentally turned off the oxygen flow to a neighbouring patient with serious consequences. It is recommended that patients should not be allowed to adjust oxygen flow, especially if there are dual outlets.

\section{Recommendation}

33. Trusts should take measures to eliminate the risk of oxygen tubing being connected to the incorrect wall oxygen outlet or to outlets that deliver compressed air or other gases instead of oxygen. Air flow meters should be removed from the wall sockets or covered with a designated air outlet cover when not in use.
Special care should be taken if twin oxygen outlets are in use. [Grade D]

\subsection{Use of humidified oxygen}

\subsubsection{Rationale for use of humidified oxygen}

The upper airway normally warms, moistens and filters inspired gases. When these functions are impaired by a pathological process or when they are bypassed by an artificial airway, it is common practice to provide humidification. The main reason for using humidification, especially with high-flow oxygen, is that it may reduce the sensation of dryness in the upper airways that oxygen can cause. However, in the non-intubated population there appears to be little scientific evidence of any benefit from humidified oxygen except that single doses of nebulised isotonic saline have been shown to assist sputum clearance and reduce breathlessness in patients with COPD. ${ }^{303} 304$ There is also evidence that humidification, when combined with physiotherapy, can increase sputum clearance in bronchiectasis. ${ }^{305}$ Randomised controlled trials of the effects of humidified high-flow oxygen on patient comfort are required.

\section{Recommendations}

34. Humidification is not required for the delivery of low-flow oxygen or for the short-term use of highflow oxygen. It is not therefore required in prehospital care. Pending the results of clinical trials, it is reasonable to use humidified oxygen for patients who require high-flow oxygen systems for more than $24 \mathrm{~h}$ or who report upper airway discomfort due to dryness. [Grade B]

35. In the emergency situation, humidified oxygen use can be confined to patients with tracheostomy or an artificial airway although these patients can be managed without humidification for short periods of time (eg, ambulance journeys). [Grade D]

36. Humidification may also be of benefit to patients with viscous secretions causing difficulty with expectoration. This benefit can be achieved using nebulised normal saline. [Grade C]
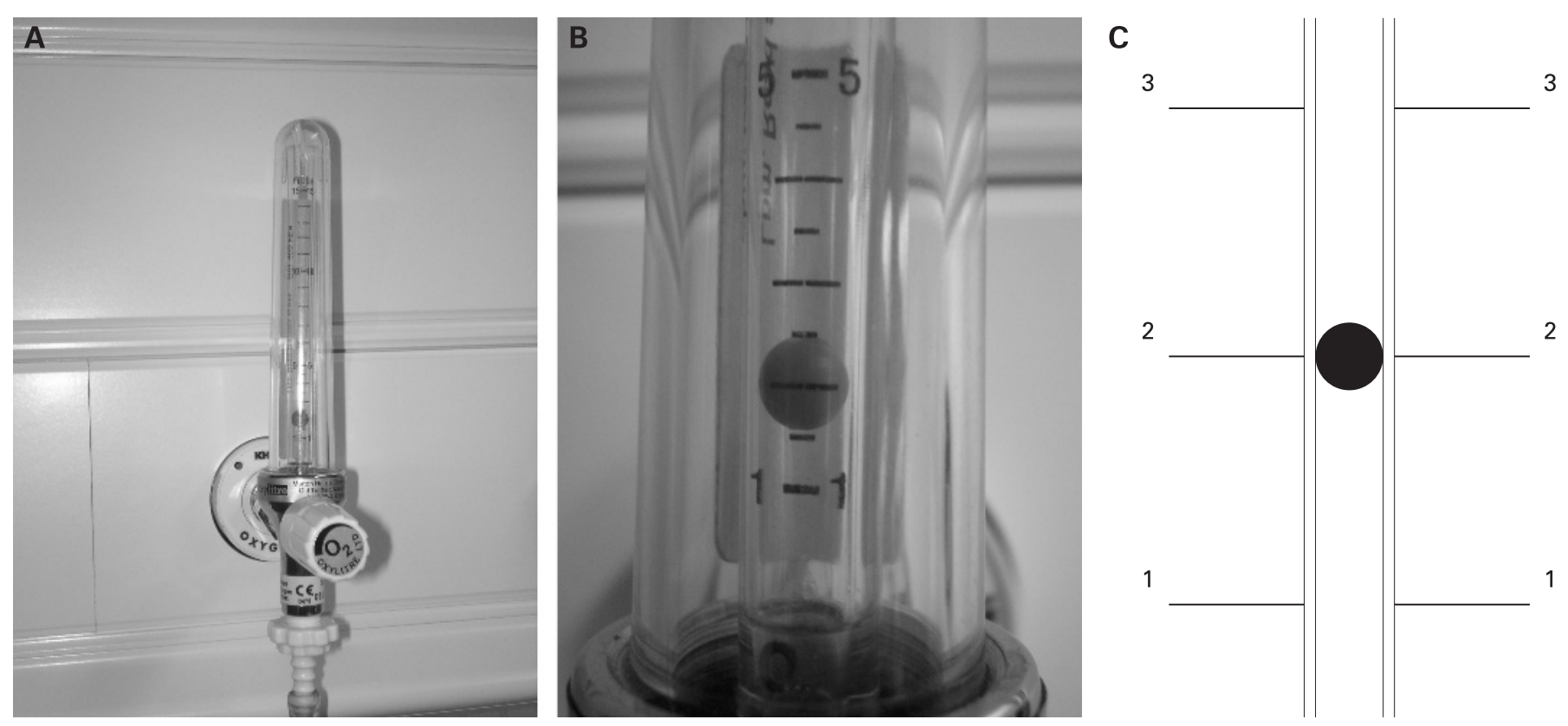

Figure 15 Flow meter showing correct setting to deliver oxygen at a rate of $2 \mathrm{l} / \mathrm{min}$. 


\subsubsection{Use of bubble humidification systems}

Humidified oxygen is widely administered in hospitals across the UK and this is presumed to alleviate nasal and oral discomfort in the non-intubated patient. Humidification of supplemental oxygen is commonly delivered by bubbling oxygen through either cold or warm sterile water before it reaches the patient. However, the effect on patient comfort is negligible. ${ }^{306} 307$ Bubble humidifiers may, however, represent an infection hazard and should not be used. ${ }^{308}$

- There is no evidence of a clinically significant benefit from "bubble bottle" systems but there is an infection risk. [Evidence level III]

\section{Recommendation}

37. Bubble bottles should not be used because there is no evidence of a clinically significant benefit but there is a risk of infection. [Grade C]

\subsubsection{Large volume nebulisation-based humidifiers}

If humidification is required, it should ideally deliver the inspired gas at a of $32-36^{\circ} \mathrm{C}$. Cold water humidifiers are simple and inexpensive but less efficient than a warm water system (about $50 \%$ relative humidity at ambient temperatures). The warm water option is more effective, targeting a relative humidity of $100 \%$, but both systems are thought to be a potential infection control risk. Warm water humidifiers are expensive and mostly confined to intensive care units and high dependency units and thus outside the scope of this guideline.

Newer humidifying systems are really "giant nebulisers" with a 1-litre reservoir of saline or sterile water and an adjustable Venturi device (fig 16). These systems are attached directly to the oxygen flow meter and connected to an aerosol mask via Flex tube. They allow delivery of precise oxygen concentrations of $28 \%, 35 \%, 40 \%$ and $60 \%$ oxygen via their Venturi device. This requires a specific oxygen flow rate as well as adjusting the Venturi nozzle on the device. It is possible to deliver $24 \%$ oxygen using a special adaptor. These large volume humidifiers have a high humidification output. The main indication for use is to assist with expectoration of viscous sputum. There are no published randomised studies involving these devices, but it has been shown that single doses of nebulised saline can assist sputum production and relieve breathlessness in patients with COPD. ${ }^{303} 304$

- Patients requiring high flow rates or longer term oxygen might benefit from a large volume oxygen humidifier device, especially if sputum retention is a clinical problem. [Evidence level III]

- In the absence of an artificial airway the decision to humidify supplemental oxygen needs to be made on an individual basis but this practice is not evidence-based. [Evidence level IV]

\subsection{Use of oxygen in patients with tracheostomy or laryngectomy}

The number of patients with a tracheostomy being cared for in a ward setting is increasing as critical care personnel use this as a method of facilitating weaning from mechanical ventilation. In the absence of a pressurised circuit, oxygen is predominantly delivered via tracheostomy mask. This is a variable performance device and delivers concentrations up to $60-70 \%$. If the patient deteriorates and requires an increased oxygen concentration exceeding the concentration that a variable performance

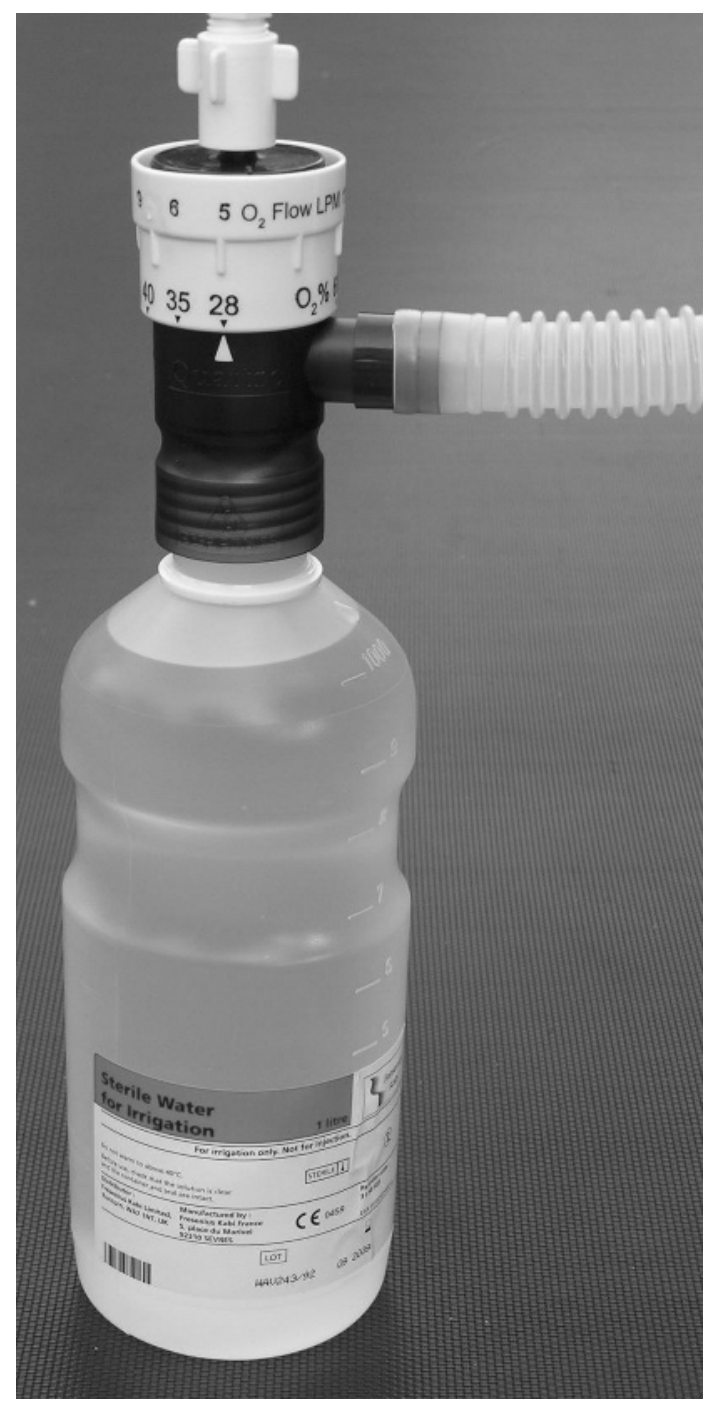

Figure 16 Large volume nebulisation-based humidifier.

interface can deliver (60-70\%), it will be necessary to seek an alternative delivery system, usually a T-piece device fitted directly to the tracheostomy tube.

With a mask system the interface will be connected to a humidification system via elephant tubing. As inserting a tracheostomy tube bypasses the patient's natural mechanisms to warm and moisturise inspired gases, it is essential to humidify any supplemental oxygen being delivered to the tracheostomised patient. This will help maintain a patent tracheostomy tube, reducing the build-up of secretions within the inner tube or the tracheostomy itself and minimising any subjective discomfort that the patient may experience.

\section{Recommendation}

38. When oxygen is required by patients with prior tracheostomy or laryngectomy, a tracheostomy mask (varying the flow as necessary) should achieve the desired oxygen saturation (tables 1-4). An alternative delivery device, usually a two-piece device fitted directly to the tracheostomy tube, may be necessary if the patient deteriorates. [Grade D] 


\subsection{Delivering oxygen to patients who require nebulised bronchodilator therapy}

\subsubsection{Nebulised bronchodilator therapy in asthma}

In patients with acute severe asthma oxygen should be used as the driving gas for the nebulised bronchodilators whenever possible at a gas flow rate of $6-81 /$ min because these patients are at risk of hypoxaemia. If the available cylinders in general practice do not produce this flow rate, an air-driven nebuliser (with electrical compressor) should be used with supplemental oxygen by nasal cannulae at $2-61 / \mathrm{min}$ to maintain an appropriate oxygen saturation level. There is some evidence that bronchodilator therapy (whether delivered by nebuliser or by metered dose inhaler) may cause pulmonary vasodilation leading to increased $\mathrm{V} / \mathrm{O}$ mismatch and reduced blood oxygen levels in acutely ill patients and in patients with stable COPD and asthma. ${ }^{309-311}$ The lowest oxygen saturation occurs not during bronchodilator therapy but about 25 min later. ${ }^{311}$ One group reported a rise in oxygen saturation during bronchodilator therapy. ${ }^{312}$

- There is some evidence that bronchodilator therapy, however given, can cause increased $\mathrm{V} / \mathrm{O}$ mismatch and reduced blood oxygen levels in acutely ill patients shortly after treatment. [Evidence level III]

10.8.2 Nebulised bronchodilator therapy for patients with COPD and other risk factors for hypercapnic respiratory failure

When an oxygen-driven nebuliser is given to patients with COPD there is a risk of hypercapnia and acidosis due to the high $\mathrm{FIO}_{2}$ which is delivered. In acute exacerbations of COPD the carbon dioxide level can rise substantially within 15 min of commencing high-dose oxygen therapy. ${ }^{102}$ When nebulised bronchodilators are given to hypercapnic patients, they should ideally be given using an electrical compressor and, if necessary, supplementary oxygen should be given concurrently by using nasal cannulae at $1-4 \mathrm{l} / \mathrm{min}$ to maintain an oxygen saturation of $88-92 \%$. If an electrical compressor is not available, compressed air can be used to drive the nebuliser but care should be taken to ensure that the nebuliser is not attached an oxygen outlet (see section 10.5.6). Once the nebulised treatment is completed, controlled oxygen therapy with a fixed concentration (Venturi) device should be reinstituted.

Compressed air is not routinely available during ambulance journeys. In this situation oxygen-driven nebulisers may be used but should be limited to 6 min for patients with known COPD because the nebuliser mask delivers a high dose of oxygen (about 60\%). Most of the effective dose from a nebuliser chamber is delivered within $6 \mathrm{~min}$ and it is known that highdose oxygen can cause significant hypercapnia in acute COPD within 15 min. ${ }^{102}$ Limiting oxygen-driven nebulisers to $6 \mathrm{~min}$ will limit the risk of hypercapnic respiratory failure while delivering most of the nebulised drug dose It must be recognised that patients with COPD may develop hypercapnia and respiratory acidosis if they are left on high-flow oxygen via a nebuliser mask system long after the nebulisation process has finished.

- High-dose oxygen therapy can cause hypercapnia within $15 \mathrm{~min}$ in acute COPD. [Evidence level III]

\section{Recommendations}

39. For patients with asthma, nebulisers should be driven by piped oxygen or from an oxygen cylinder fitted with a high-flow regulator capable of delivering a flow rate of $>61 / \mathrm{min}$. The patient should be changed back to his/her usual mask when nebuliser therapy is complete. If the cylinder does not produce this flow rate, an air-driven nebuliser (with electrical compressor) should be used with supplemental oxygen by nasal cannulae at $2-61 / \mathrm{min}$ to maintain an appropriate oxygen saturation level. [Grade D]

40. When nebulised bronchodilators are given to patients with hypercapnic acidosis, they should be driven by compressed air and, if necessary, supplementary oxygen should be given concurrently by nasal cannulae at $2-4 \mathrm{l} / \mathrm{min}$ to maintain an oxygen saturation of $88-92 \%$. The same precautions should be applied to patients who are at risk of hypercapnic respiratory failure prior to the availability of blood gas results. Once the nebulised treatment is completed for patients at risk of hypercapnia, controlled oxygen therapy with a fixed concentration (Venturi) device should be reinstituted. [Grade D]

- During ambulance journeys, oxygen-driven nebulisers may be used in the absence of an air-driven compressor system. If oxygen is used, its use should be limited to $6 \mathrm{~min}$ for patients with known COPD. This will deliver most of the nebulised drug dose but limit the risk of hypercapnic respiratory failure (see recommendation 27).

\section{SECTION 11: PRESCRIPTION, ADMINISTRATION AND MONITORING OF OXYGEN THERAPY}

\subsection{Safe prescription and administration of oxygen}

11.1.1 Legal status of medical oxygen: does it need a prescription? Medical oxygen is a drug. However, the legal status of oxygen in the UK is that of a medicinal product on the General Sales List (Medicines and Healthcare Products Regulatory Agency, personal communication). This means that the sale or dispensation of oxygen does not technically require a prescription because it is not a "prescription only medicine". This status was conferred for practical reasons to facilitate the use of oxygen in the domiciliary setting where the distribution system no longer involves pharmacies. However, the use of oxygen by paramedics, nurses, doctors, physiotherapists and others in emergency situations is similar to the use of all other medicinal products by these people. Clinical governance requires that the intentions of the clinician who initiates oxygen therapy should be communicated clearly to the person who actually administers oxygen to the patient and an accurate record must be kept of exactly what has been given to the patient. In this respect, oxygen is in the same category as paracetamol, aspirin, ibuprofen, antihistamines, anti-emetics and many other medicines that do not require a prescription if purchased by a patient for his/her own use but do require accurate documentation if administered by a health professional to a patient. In healthcare settings, all of these medicines are conventionally recorded on a "prescription chart" or "drug kardex" alongside drugs in the "prescription only" category such as antibiotics.

A hospital "prescription chart" is a document in which doctors and other prescribing clinicians make a list of all drugs and medicinal products required by a patient and where nurses and other clinicians make a record of all drugs that have been administered to the patient. These documents are also used by hospital pharmacies to dispense medicines but, for technical reasons, these hospital documents (or the equivalent documents in primary care settings or the Patient Report Form (PRF) or ePRF used by the ambulance services) do not have the same 
legal status as a prescription that is given to a patient to take to a community pharmacist for the purposes of dispensing a medicine. The "prescription" of oxygen in acute healthcare settings is therefore not technically a prescription for two reasons: (1) oxygen is not a prescription only medicine; and (2) a hospital drug chart is not truly a prescription. The second issue applies to all medicines used in healthcare settings, not just to oxygen. However, the words "prescription" or "prescription chart" are widely used to describe the documents which record the use of medicines in UK hospitals and other healthcare settings. For this reason, the present guideline will use the term "prescribe" and "prescription chart" for all orders for oxygen use. The important issue is that there must be a clear written record of all medicinal products, including oxygen, which are given to a patient by healthcare professionals. Ideally, this document should be prepared at the time when oxygen therapy is commenced. In emergencies, however, clinicians will treat the patient first and subsequently make written records of all treatments given, including oxygen therapy.

11.1.2 Reasons to prescribe oxygen therapy (see sections 5 and 6) Oxygen is prescribed for hypoxaemic patients to increase oxygen tension and decrease the work of breathing necessary to maintain a given $\mathrm{PaO}_{2}$. The concentration of oxygen required depends on the condition being treated; an inappropriate concentration may have serious or even lethal effects. Appropriate target saturation ranges for common medical emergencies are given in section 8 of this guideline and in tables 1-4. Each hospital should have an agreed patient group directive for oxygen prescribing to allow staff to adjust oxygen delivery devices and to give oxygen in emergency situations prior to the availability of a prescription.

\subsubsection{Implementing an oxygen prescription policy}

Oxygen prescriptions should include starting dose and initial mode of delivery and whether the oxygen therapy should be continuous or as-required. The most important aspect of the prescription is to give a target range. The clinicians who administer oxygen (usually nurses, midwives or physiotherapists) should be trained and empowered to adjust the oxygen dose upwards and downwards as necessary to maintain the patient in the target saturation range. This will require all hospitals to have an agreed oxygen administration programme with universal access to educational materials about oxygen administration. The clinicians who monitor the oxygen saturation (often health care assistants) should be trained to inform those who have been trained to administer oxygen if the oxygen saturations fall above or below the target saturations. Those doing the monitoring should also understand the importance for the patient of keeping in the target range. Implementing this policy will require all hospitals to have agreed patient group directives and training programmes for all clinical staff and regular training programmes in the safe use of oxygen and audit of outcomes.

\subsubsection{Administration and monitoring of oxygen therapy}

The appropriate device should be used to provide the prescribed oxygen and the effects should be monitored using pulse oximetry, monitoring of respiration rate and close observation of the patient. Arterial or capillary blood gas analysis should be repeated if clinical progress is not satisfactory and in all cases of hypercapnia and acidosis.

\subsubsection{Education of health professionals}

The clinician or healthcare professional administering the oxygen therapy should be aware of the hazards of hypoxaemia and hyperoxaemia and the signs and symptoms of inadequate or excessive oxygen delivery.

\subsubsection{How to prescribe oxygen effectively}

In the past, oxygen was often not prescribed at all or prescribed on a standard hospital drug chart as "oxygen". It was unusual for the prescription to include full details of what device to use, what flow rate(s) to administer and whether the prescription was for a fixed dose of oxygen or to aim at a specific oxygen saturation target. ${ }^{2-8}$

It has been shown that a purpose-designed oxygen prescription sheet can improve oxygen prescribing in the short term, but experience has shown that free-standing oxygen prescription charts are often forgotten and unused.

Recent audit studies by members of the guideline group (not yet published) have shown improved standards of prescribing with the use of a preprinted section for oxygen use in all hospital drug charts. This system was further enhanced by setting a desired saturation range for each patient. Suggested target saturations for common medical conditions are given in sections 8 and 9 of this guideline. It is important that healthcare professionals where oxygen is administered are familiar with the optimal saturation ranges for common conditions (summary guideline for hospitals; web appendix 2), and it is also important that those delivering the oxygen are familiar with the equipment in use and the best types of device to deliver low, medium and high-dose oxygen therapy. Chart 3 in fig 17 shows a working example of a preprinted oxygen section for a hospital prescription chart, and charts 1 and 2 (figs 1 and 2) give advice to prescribers and advice to those delivering oxygen on wards.

The safe use of oxygen includes careful consideration of the appropriate delivery device (mask, cannulae, etc) together with an appropriate source of oxygen and an appropriate oxygen flow rate.

- For hypoxaemic patients, oxygen therapy should continue during other treatments such as nebulised therapy (see recommendations 27,39 and 40 ).

Recommendations for safe prescribing and safe administration of oxygen

41 Every healthcare facility should have a standard oxygen prescription document or, preferably, a designated oxygen section on all drug prescribing cards. [Grade C]

- Oxygen saturation should be measured in all breathless patients and supplemental oxygen should be given to all breathless hypoxaemic patients and to all critically ill patients. Oxygen saturation should be measured under as optimal conditions as possible e.g. nail varnish should be removed (see recommendation 9).

- Clinicians should assess the clinical status of the patient prior to prescribing oxygen and the patient's condition should be reassessed frequently during oxygen use (see recommendations 7-9).

42 A prescription for oxygen should always be written, except in life-threatening emergencies when it must be started immediately. [Grade D]

43 Doctors and other prescribers should prescribe oxygen using a target saturation range (sections 5 , 
Oxygen The method and rate of oxygen delivery should be altered by nurses or other healthcare professionals in order to achieve the target saturation range as per hospital guideline. For most conditions, oxygen should be prescribed to achieve a target saturation of $94-98 \%$ (or $88-92 \%$ for those at risk of hypercapnic respiratory failure). The nurse should sign this prescription chart on every drug round. The delivery device and flow rate should be recorded alongside the oxygen saturation on the bedside observation chart or Early Warning Score chart.

*Saturation is indicated in almost all cases except for palliative terminal care.

\section{Drug OXYGEN}

Circle target oxygen saturation

$88-92 \% \quad 94-98 \% \quad$ Other

Starting device/flow rate

PRN/continuous (refer to $\mathrm{O}_{2}$ guideline)

Tick here if saturation not indicated *

Date and signature

Print name

\section{Drug OXYGEN}

Circle target oxygen saturation

$88-92 \% \quad 94-98 \% \quad$ Other

Starting device/flow rate

PRN/continuous (refer to $\mathrm{O}_{2}$ guideline)

Tick here if saturation not indicated *

Date and signature

Print name
Date administered

\begin{tabular}{|l|l|l|l|l|l|l|l|l|l|l|l|l|l|l|}
\hline & & & & & & & & & & & & & & \\
\hline 06 & & & & & & & & & & & & & & \\
\hline 09 & & & & & & & & & & & & & & \\
\hline 14 & & & & & & & & & & & & & & \\
\hline 18 & & & & & & & & & & & & & & \\
\hline 22 & & & & & & & & & & & & & & \\
\hline
\end{tabular}
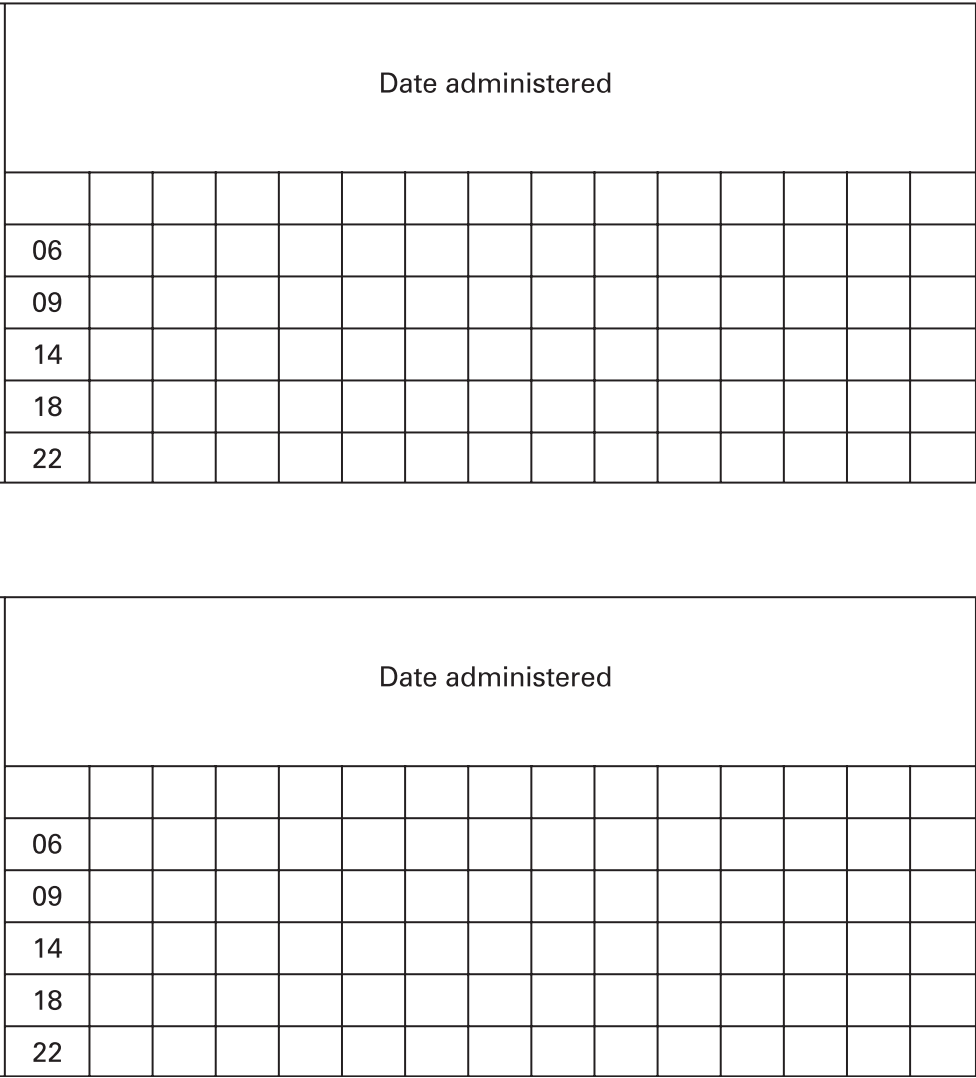

Figure 17 Chart 3: Working example of oxygen section for hospital prescription charts (two panels are required on the prescription chart because oxygen may change from continuous to "as required" (PRN) as a patient improves).

6, 8, 9 and 11) and sign the drug chart (chart 3, fig 17). [Grade D]

44 In all situations where repeated blood gas measurements are required (see recommendations 13, 60 and 66), they should be measured as soon as possible, usually within $30 \mathrm{~min}$, to determine if the proposed target saturations are appropriate. [Grade D]

45 Adjustments should only be made by staff who have been trained to administer oxygen if the oxygen saturation falls below the prespecified range and the dose should be reduced if the saturation rises above this range. If the monitoring of oxygen saturation is performed by other staff (eg, health care assistants), they should inform staff who are trained to administer oxygen if the oxygen saturation is above or below the target saturation. [Grade D]

All clinicians prescribing oxygen should have appropriate training and access to written or electronic oxygen prescribing guidelines based on this national guideline. [Grade D]
47 Every hospital should have a training programme to ensure that clinical staff are familiar with the hospital's oxygen administration policies. [Grade D]

11.1.7 How to use the oxygen section in a drug chart (chart 3 , fig 17) Recommendations

- Oxygen should be prescribed on the drug chart using a target saturation range (see recommendations $1-5$ and $41-$ 47).

- In most emergency situations, oxygen is given to patients immediately without a formal prescription. The lack of a prescription should never preclude oxygen being given when needed in an emergency situation. However, a subsequent written record must be made of what oxygen therapy has been given to every patient in a similar manner to the recording of all other emergency treatment (see recommendation 42).

48 Nurses should sign the drug chart at every drug round and check that the patient is receiving oxygen therapy. This is to check that the patient is within the target saturation and also to check whether 
weaning and discontinuation should be instituted (see recommendations 73-76). [Grade D]

49 Most patients are prescribed continuous oxygen. However, some patients may be prescribed oxygen as required (PRN). In this scenario, if patients are on air at the time of the drug round, nurses should still sign the drug chart but the observation chart should be filled in using the code A for air (see chart 4, fig 18). [Grade D]

\subsection{Starting oxygen therapy}

Safe prescribing and safe administration of oxygen are closely linked. In emergencies oxygen therapy should be started immediately and prescribed as soon as possible. In all other situations oxygen should be prescribed in accordance with the standards described in section 11.1 before administration is commenced. The healthcare professional who administers the oxygen therapy (usually a nurse or physiotherapist) should be fully trained and should follow local or national protocols as described in section 11.1.

\section{Recommendations}

- The administering healthcare professional should note the oxygen saturation before commencing oxygen therapy (see recommendation 9).
50 The healthcare professional should commence oxygen therapy using an appropriate delivery system and flow rate as specified in sections 8, 9 and 10 of this guideline. The target oxygen saturation and whether the patient is having continuous oxygen, PRN oxygen or no oxygen therapy should be added on the respiratory section of the observation chart. [Grade D]

51 Whenever possible, patients should be given an oxygen information sheet (example in web appendix 5 of this guideline). [Grade D]

\subsection{Monitoring oxygen therapy}

\subsubsection{Pulse oximeters}

Pulse oximetry should be available to all healthcare staff managing patients receiving oxygen therapy and they should be trained in their use (see section 7 for technical and practical information regarding oximeter use). Clinicians should be aware that pulse oximetry gives no information about the $\mathrm{PCO}_{2}$ or $\mathrm{pH}$ and most pulse oximeters are unreliable when a patient's $\mathrm{SpO}_{2}$ falls below about $85 \%$. Pulse oximetry is dependent on pulsatile flow, and it may not be possible to achieve a satisfactory oximeter reading in patients with cold hands, especially those with severe Raynaud's phenomenon due to collagen vascular diseases (which may also cause hypoxic lung disease). The readings may also be affected by shock, skin pigmentation, nail

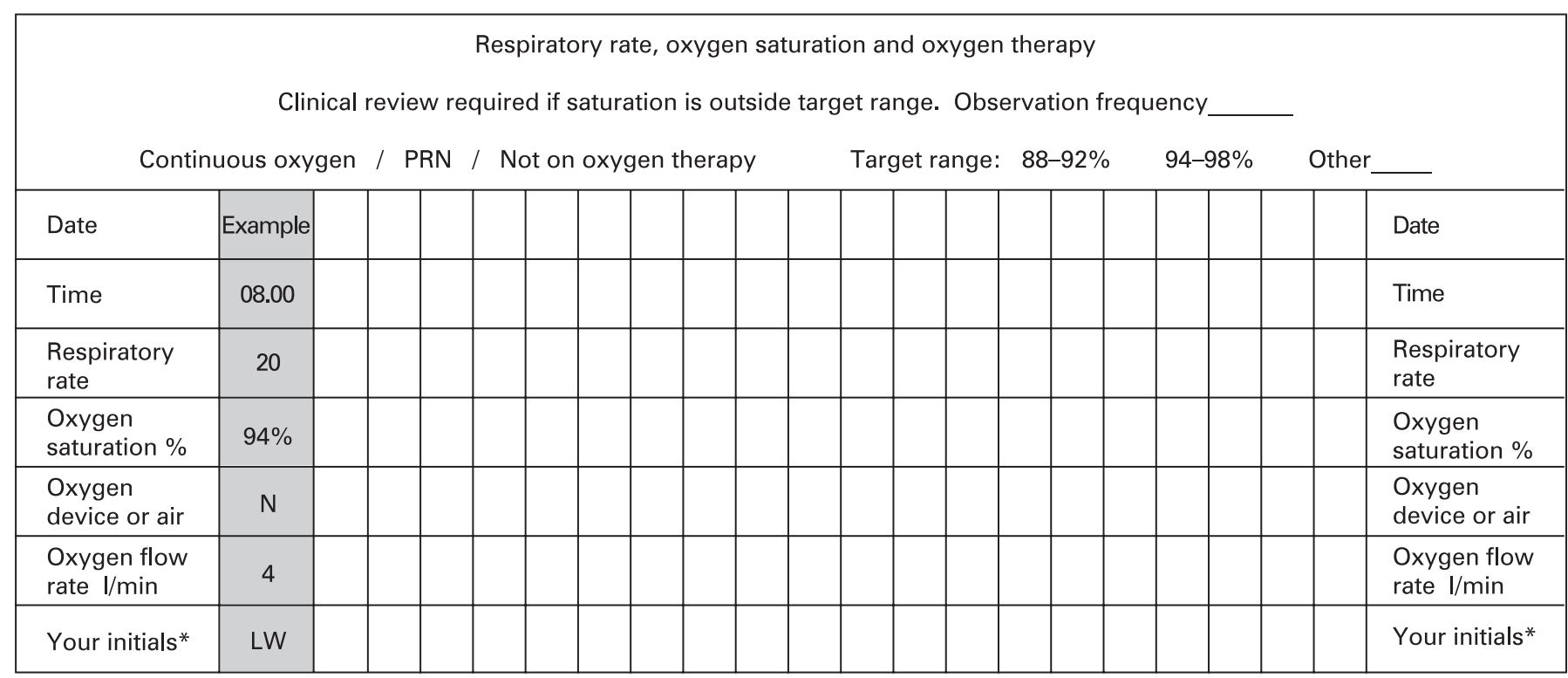

${ }^{*}$ All changes to oxygen delivery systems must be initialled by a registered nurse or equivalent.

If the patient is medically stable and in the target range on two consecutive rounds, report to a registered nurse to consider weaning off oxygen.

${ }^{*}$ Codes for recording oxygen delivery on observation chart

\begin{tabular}{|c|c|c|c|c|c|}
\hline A & \multicolumn{3}{|c|}{ Air (not requiring oxygen, or weaning or on "PRN" oxygen) } & $\mathrm{H} 28$ & $\begin{array}{l}\text { Humidified oxygen at } 28 \% \\
\text { (also } \mathrm{H} 35, \mathrm{H} 40, \mathrm{H} 60 \text { for humidified oxygen at } 35 \%, 40 \%, 60 \% \text { ) }\end{array}$ \\
\hline $\mathrm{N}$ & \multirow{2}{*}{\multicolumn{3}{|c|}{ Nasal cannulae }} & RM & Reservoir mask \\
\hline & & & & TM & Tracheostomy mask \\
\hline SM & \multicolumn{3}{|l|}{ Simple mask } & & $\begin{array}{l}\text { Patient on CPAP system } \\
\text { Patient on NIV system }\end{array}$ \\
\hline V24 & Venturi $24 \%$ & Ventri $28 \%$ & Venturi $35 \%$ & OTH & Other device: \\
\hline V40 & Venturi $40 \%$ & Venturi $60 \%$ & & & (specify which) \\
\hline
\end{tabular}

Figure 18 Chart 4: Working example of respiratory section of observation chart for hospital use. 
varnish, etc (see section 7). It is essential to record the oxygen delivery system alongside the oximetry result.

- All measurements of oxygen saturation should be recorded in the observation chart along with the code for the oxygen delivery system that is being used. If the patient is breathing air at the time of the observation, this should also be recorded in the chart (see recommendation 9 and chart 4 (fig 18))

\subsubsection{Arterial or arteriolised capillary blood gases}

- Arterial or arteriolised capillary blood gases should be measured and the inspired oxygen concentration noted on arrival at hospital (or at the time when oxygen therapy becomes necessary) for most patients requiring emergency oxygen therapy (see recommendations 11-13).

- Blood gas measurements should be repeated in all critically ill patients and in many other cases according to the response to treatment (see recommendations 11-13).

\subsubsection{Physiological monitoring: "track and trigger" systems}

- Early Warning Scoring systems (EWS or mEWS) are useful for monitoring patients. [Evidence Level III]

- Tachypnoea is a sensitive indicator of deteriorating respiratory function. [Evidence Level III]

- All acutely ill patients should have physiological monitoring using Early Warning Scores or a similar physiological assessment system in addition to pulse oximetry (see recommendation 8 ).

11.3.4 Monitoring during the first hour of oxygen therapy Recommendations

52 All patients should have their oxygen saturation observed for at least five minutes after starting oxygen therapy. [Grade D]

53 If the oxygen saturation should fall below the target saturation and the patient is unstable, medical advice should be sought. [Grade D]

54 If the oxygen saturation is above the target saturation range and the patient is stable, the delivery system and oxygen flow rate should be reduced accordingly. [Grade D]

55 Patients who have a target saturation of $88-92 \%$ should have their blood gases measured within 30 $60 \mathrm{~min}$. This is to ensure that the carbon dioxide level is not rising. This recommendation also applies to those who are at risk of developing hypercapnic respiratory failure but who have a normal $\mathrm{PaCO}_{2}$ on the initial blood gas measurement. [Grade D]

56 Stable patients whose oxygen saturation is within their target saturation range of $94-98 \%$ do not need repeat blood gas measurements within 30$60 \mathrm{~min}$ if there is no risk of hypercapnia and acidosis and may not need any further blood gas measurements. [Grade D]

\subsubsection{Subsequent monitoring}

The exact requirements for monitoring will depend on the clinical condition of each patient. Saturations are usually measured after $1 \mathrm{~h}$ of oxygen therapy and then 4-hourly. Stable patients should be monitored four times a day. However, critically ill patients will require continuous monitoring of oxygen saturation and other physiological measurements.

\section{Recommendations}

57

58

59

65

\subsubsection{When to increase oxygen therapy}

In most instances, failure to achieve the desired oxygen saturation is due to the severity of the patient's illness but it is worth checking that the oxygen delivery device and the oxygen flow rate are correct. If the oxygen is being delivered from a cylinder, clinicians should check the labelling of the cylinder to ensure that it is an oxygen cylinder and checks should be made to ensure that the cylinder is not empty or nearempty.

\section{Recommendations}

68 If a patient's oxygen saturation is lower than the prescribed target range, first check all aspects of 
the oxygen delivery system for faults or errors. [Grade D]

69

If a patient's oxygen saturation is consistently lower than the prescribed target range, there should usually be a medical review and the oxygen therapy should be increased according to an agreed written protocol. [Grade D]

70 The patient should be observed for $5 \mathrm{~min}$ after oxygen therapy has been increased. [Grade D]

71

If the oxygen saturation fails to rise following 5$10 \mathrm{~min}$ of increased oxygen therapy or if there is clinical concern following medical review, then blood gas measurements should be repeated. [Grade D]

72 If the target saturation is in the $88-92 \%$ range, blood gas measurements should be repeated at 30$60 \mathrm{~min}$ after any increase in oxygen therapy to ensure that the carbon dioxide level is not rising. [Grade D]

\subsubsection{When to lower oxygen therapy}

Most conditions which require supplemental oxygen therapy will improve with treatment and it will then be possible to reduce the amount of oxygen administered to the patient. Improvement will usually be confirmed by observing an improving oxygen saturation and a reduction in the physiological score on the mEWS observation chart as discussed in section 7 .

\section{Recommendations}

73 Lower the oxygen dose if the target saturation is higher than the prescribed range. [Grade D]

74 Lower the oxygen dose if the patient is clinically stable and the oxygen saturation has been in the upper zone of the target range for some time (usually 4-8 h). [Grade D]

Saturations should be observed for $5 \mathrm{~min}$ following a change of oxygen therapy or rechecked after 5 min on the lower dose of oxygen. [Grade D]

If the target saturation is maintained, the new delivery system and flow should be continued. Repeat blood gas measurements are not required. If the patient is stable the process can be repeated and the patient can eventually be weaned off oxygen (see section 12). [Grade D]

\section{SECTION 12: WEANING AND DISCONTINUATION OF OXYGEN THERAPY}

In most acute illnesses, oxygen therapy will be reduced gradually as the patient recovers and oxygen therapy can be discontinued once the patient can maintain a saturation of 94 98\% while breathing air (or the patient's baseline oxygen saturation level if known). However, some patients may be continued on oxygen therapy to palliate breathlessness, often on a PRN basis (as required, not continuous). Some patients may have episodic hypoxaemia during recovery from acute illness (eg, patients with COPD with intermittent mucus plugging) and some convalescent patients may be comfortable at rest with a normal oxygen saturation but may desaturate and become breathless when they start to mobilise. There is no evidence that oxygen, either before or after exercise, is of benefit to non-hypoxaemic patients either by relieving breathlessness or by shortening length of stay in hospital. More research in this area is needed.

Some patients with chronic lung diseases will already be established on long-term oxygen therapy and should be tapered slowly to their usual maintenance dose of oxygen.

A small number of patients who have suffered major respiratory or cardiac injury may require a prescription for home oxygen to permit safe discharge from hospital. However, many patients with COPD exacerbations may have a low $\mathrm{PaO}_{2}$ on discharge from hospital but a reasonable $\mathrm{PaO}_{2}$ at a subsequent clinic visit, so decisions about long-term oxygen should not be made on the basis of blood gas measurements made during acute exacerbations of COPD (see Royal College of Physicians clinical guideline for domiciliary oxygen services ${ }^{313}$ ).

\subsection{How to discontinue oxygen therapy for stable patients}

\section{Recommendations}

- Reduce oxygen therapy gradually for stable patients (see section 11.3.7)

77 Most stable convalescent patients will eventually be stepped down to $2 \mathrm{1} / \mathrm{min}$ via nasal cannulae prior to cessation of oxygen therapy. Patients at risk of hypercapnic respiratory failure may be stepped down to $1 \mathrm{l} / \mathrm{min}$ via nasal cannulae or a $24 \%$ Venturi mask at $21 / \mathrm{min}$ as the lowest oxygen dose prior to cessation of oxygen therapy. [Grade D]

78 Oxygen therapy should be stopped once a patient is clinically stable on low-dose oxygen and the oxygen saturation is within the desired range on two consecutive observations. Oxygen should also be stopped if the patient is on a written protocol of timed oxygen (eg, postoperatively). [Grade D]

79 Oxygen saturation on room air should be monitored for $5 \mathrm{~min}$ after stopping oxygen therapy. If it remains in the desired range it should be rechecked at $1 \mathrm{~h}$. [Grade D]

80 If the oxygen saturation and physiological "track and trigger" score (eg, mEWS) is satisfactory at $1 \mathrm{~h}$, the patient has safely discontinued oxygen therapy but saturation and physiology should continue to be monitored on a regular basis according to the patient's underlying clinical condition. [Grade D]

81 If the saturation falls on stopping oxygen therapy, recommence the lowest dose that maintained the patient in the target range and monitor for $5 \mathrm{~min}$. If this restores the saturation into the target range, continue oxygen therapy at this level and attempt discontinuation of oxygen therapy again at a later date provided the patient remains clinically stable. [Grade D]

82 If a patient requires oxygen therapy to be restarted at a higher dose than before to maintain the same target saturation range, the patient should have a clinical review to establish the cause for this deterioration. [Grade D]

83 Some patients may have episodic hyoxaemia (eg, after minor exertion) after they have safely discontinued continuous oxygen therapy. If these patients require intermittent oxygen therapy, they should have a prescription for oxygen as required (PRN) but PRN oxygen should not be routinely prescribed for those who have stopped continuous oxygen therapy. [Grade D] 
Cross oxygen off the drug chart when oxygen discontinued (and sign to confirm discontinuation). [Grade C]

\section{SECTION 13: OUTCOMES AND AUDIT \\ 13.1 Audit}

Successful audits of oxygen prescription and use have been carried out at the pilot hospitals in Salford and Southend and the use of oxygen prescription linked to "track and trigger" or mEWS scoring systems has also been audited in conjunction with the outreach teams.

One year after publication of this guideline the Emergency Oxygen Guideline Committee will organise a UK-wide audit to establish how many hospital, PCT providers and ambulance services have introduced the guideline. Audit of oxygen use within individual trusts will also be strongly encouraged.

\subsection{Audit of compliance with guidelines}

It is recommended that all users of oxygen will audit their own practice against the suggested optimal practice suggested in this guideline. This applies especially to high-frequency users such as ambulance services, emergency departments and medical wards.

\section{"On the job audit"}

Regular reviews of the drug card and observation chart on medical rounds at the pilot sites have been very important in the successful introduction of this policy. This has provided instant feedback for doctors, nurses and health care assistants and has produced successful change.

\section{Whole hospital and ward audits}

An audit tool has been developed at the pilot sites (web appendix 7). This has been successfully used hospital-wide and on individual wards. It is suggested that audits are done soon after the introduction of the policy.

Other audit questions could include the following:

- Does the trust have an oxygen prescribing policy based on this guideline?

- Is this policy known to all staff and available at all times on the trust intranet?

- Was oxygen prescribed for every patient who was using oxygen at the time of the audit?

- Did every patient have a target oxygen saturation specified (and was this target appropriate)?

- Was oxygen given in accordance with the present guideline?

- Was FEV 1 measured in patients with a diagnosis of COPD (either acutely or prior to discharge and subsequently during follow-up)?

\section{SECTION 14: DISSEMINATION AND IMPLEMENTATION OF THE GUIDELINE}

\subsection{Dissemination}

Dissemination of this guideline will be encouraged and supported strongly by the societies involved in the production of the guideline. It is hoped that each specialist Society or College will alert members to the key recommendations in this guideline. Copies will be sent to the chief executives, medical directors and head nurses of all hospital and primary care trusts and ambulance trusts and voluntary and private ambulance providers in the UK and also to the directors of education at nursing schools, medical schools and at Education for Health (National Respiratory Training Centre).

\subsection{Local guidelines}

It is recognised that many trusts tend to modify national guidelines for local use. Short "user friendly" versions of the guideline website for acute hospitals, ambulance services and general practice are shown in appendices 2 and 4 on the BTS website (www.brit-thoracic.org.uk).

Educational materials will be made available on the BTS website to include guideline summaries and flow charts in addition to teaching slides. It is hoped that the shortened version of this guideline (or a customised local version) will be made available on the website of every NHS trust.

\subsection{Local oxygen policy}

It is usual for a new policy to be presented to the local policy committee. A specimen example of a local policy is available in web appendix 3 to help with the production of this policy in individual trusts.

\subsection{New prescription chart}

The introduction of the guideline will require a new "oxygen section" in the prescription chart in all hospitals. A specimen example is available in chart 3 (fig 17). From experience at the two pilot sites, it is recommended that oxygen should be at the start of the prescription chart because so many patients are prescribed oxygen. The oxygen prescription may be missed if it is placed in another part of the drug chart.

\subsection{Staff education}

Medical staff education will be required before the introduction of the guideline and regularly thereafter. Teaching slides are available on the BTS website. It is thought that these would be suitable for FY1, FY2 and specialty training lectures and other educational material. They are also suitable for undergraduate medical education. Nursing staff and nursing students will also require education as will physiotherapists, pharmacists, midwives and other clinicians who use oxygen. Lecture studies have also been produced for this purpose and are available on the BTS website. It is suggested that small groups of 5-10 nurses from wards are taught in sessions lasting 30 min each day before the introduction of the guideline locally. This has been found to be more successful than relying on training days. We believe that this would take too long to train enough staff adequately. Slides are available for ambulance staff and primary care staff on the BTS website.

\subsection{Local champions}

The guideline committee has tried to identify local champions in hospitals, PCT providers and ambulance services who will help to introduce these guidelines. The local champions are listed in web appendix 13 on the BTS website. It is hoped that the champions will help organise the introduction of a local guideline and oxygen policy, a new prescription chart and help organise staff education.

Clinical governance leads will also need to become committed to the implementation of the emergency oxygen guideline and audit of this process.

\subsection{Benefits of nationwide implementation}

One major benefit of nationwide implementation will be that, when staff transfer between organisations, they will be familiar with the oxygen prescription and administration system. 


\section{SECTION 15: AREAS REQUIRING FURTHER RESEARCH}

Because of the life and death nature of many conditions for which emergency oxygen therapy is used, it seems that clinicians have been wary of conducting controlled trials of oxygen therapy for most of the commoner indications. It is worrying that the few existing trials of oxygen therapy given to non-hypoxaemic patients in common conditions such as heart attacks, strokes and difficult labour have failed to show benefit, and there have been suggestions of possible harm in these trials despite the near universal use of oxygen for such conditions in the past.

Further research is required in many areas including:

- Use of oxygen in myocardial infarction.

- Use of oxygen in unstable coronary syndromes.

- Use of oxygen in chest pain of presumed cardiac origin.

- Use of oxygen in obstetric emergencies.

- Benefits of alert cards and personalised oxygen masks for patients with prior hypercapnic respiratory failure.

- Possible benefits of alert cards for all patients with COPD.

- Clinical outcomes of patients exposed to hyperoxia.

- Studies to determine if different types of oxygen mask can affect clinical outcomes.

- Comparisons of nasal cannulae and simple face masks, especially after abdominal surgery.

- Studies to determine if implementation of this guideline improves patient outcomes.

- Prospective studies to establish the ideal target saturation range in patients with exacerbated COPD; for example, should the target range be $88-92 \%$ or slightly lower or slightly higher for optimal outcome?

- Prospective studies of the effect of oxygen in nonhypoxaemic patients with major trauma and head injury.

- Audit studies of survival outcomes in patients given oxygen therapy.

- Oxygen levels and outcomes in a wide range of conditions.

- Use of oxygen PRN or as required for relief or breathlessness in non-hypoxaemic patients with acute illness.

- Effects of humidified high-flow oxygen on patient comfort.

- Effects of increased flow rates from Venturi masks on patient comfort and oxygen saturation.

- Use of nasal cannulae to deliver low-dose oxygen therapy (eg, nasal cannulae at $0.5-1.0 \mathrm{l} / \mathrm{min}$ compared with $24 \%$ and $28 \%$ face masks).

- Clinical trials of efficacy and patient tolerance using nasal cannulae at higher flow rates such as $6-101 / \mathrm{min}$.

- Effect of cigarette smoking on blood levels of oxygen and carbon dioxide in acute illness.

- Randomised trial of "precautionary use" of oxygen in critical illness compared with a conservative policy of monitoring carefully and giving oxygen only if the saturation falls below the target range.

- Studies to compare the outcome of oxygen therapy in critical illness, comparing the North American target saturation of $>90 \%$ with the higher target saturations in this guideline (94-98\%).

\section{SECTION 16: MEMBERSHIP OF WORKING PARTY AND AUTHORSHIP}

\subsection{Membership of Working Party}

Physicians in general/respiratory medicine: R O'Driscoll, Salford (Co-Chair), British Thoracic Society; A Davison, Southend (CoChair), British Thoracic Society; M Elliott, Leeds, Royal College of Physicians; Respiratory physicians: L Howard, London, British Thoracic Society; J Wedzicha, London, British Thoracic Society and Editor of Thorax; Emergency medicine: K Mackway-Jones, Manchester, British Association for Emergency Medicine; Emergency physician (EAU): P Jenkins, Norwich, Society for Acute Medicine (SAMUK); Intensive care: R Kishen, Salford, Intensive Care Society; General practitioner: M Levy, London, Royal College of General Practitioners and GP in Airways Group (GPIAG); Nurse: S Perrott, Cambridge, Royal College of Nursing; Physiotherapist: L Mansfield, Newcastle upon Tyne, Chartered Society of Physiotherapy; ARTP representative: A Evans, North Staffs, Association for Respiratory Technology and Physiology; Patient/lay representative: S Panizzo, London, Nominated by Royal College of Physicians; Ambulance service representatives: F Moore, London, Joint Royal Collages Ambulance Liaison Committee; D Whitmore, London, British Paramedic Association Senior Clinical Advisor to the Medical Director, LAS; Cardiologist: S Gibbs, London, British Cardiovascular Society; Anaesthetist: B Martin, London, Royal College of Anaesthetists; Obstetrician: K Hinshaw, Sunderland, Royal College of Obstetricians and Gynaecologists.

\subsection{Authorship of sections of the guideline}

The outline of the guideline was developed and refined by the entire group at various meetings and email discussions as described in section 2 and each section was edited by all group members, but the work of preparing the main draft for each section was divided as follows:

\subsubsection{Main text of guideline}

1. Introduction: R O'Driscoll, A Davison

2. Methodology: R O'Driscoll, A Davison

3. Normal values and definitions: R O'Driscoll, A Davison, M Elliott, L Howard

4. General blood gas physiology: L Howard, R O'Driscoll, A Davison

5. Advanced blood gas physiology: L Howard, R Kishen, $M$ Elliott

6. Hypoxia, hyperoxia, etc: L Howard, R O’Driscoll, A Davison, R Kishen

7. Clinical/laboratory assessment: R O'Driscoll, M Elliott

8. Hospital settings: R O'Driscoll

9. Prehospital settings: A Davison, D Whitmore, F Moore, M Levy

10. Practical aspects: A Evans, L Mansfield, S Perrott, L Howard

11. Prescription of oxygen: A Davison, S Perrott, R O'Driscoll

12. Weaning and discontinuation: A Davison, R O'Driscoll

13. Outcomes and audit: R O'Driscoll, A Davison

14. Dissemination/implementation: A Davison, R O'Driscoll

15. Areas requiring further research: R O'Driscoll, A Davison

16.2.2 Web appendices (available on BTS website: www.britthoracic.org.uk)

1. Summary of recommendations: whole group

2. Summary of Guideline for hospital use: A Davison/ R O'Driscoll

3. Example of local oxygen policy: N Linaker, L Ward, R Smith, R O'Driscoll, A Davison

4. Summary for prehospital settings: A Davison, R O'Driscoll, D Whitmore, M Levy 
5. Patient information sheet: L Ward, A Davison, R O'Driscoll, S Panizzo (with feedback from patients)

6. Summary of prescription, etc: A Davison, R O'Driscoll

7. Example of audit tool: L Ward, R O'Driscoll, A Davison,

8. Lecture for doctors: A Davison, R O'Driscoll

9. Teaching aids for nurses: L Ward, A Davison, R O'Driscoll

10. Key points for hospital managers and oxygen champions: R O'Driscoll, A Davison

11. Key points for PCT managers: A Davison, R O'Driscoll

12. Key points for ambulance service managers: D Whitmore, A Davison, R O'Driscoll

13. List of NHS Trusts with oxygen champions: A Davison

14. Details of search methodology: R O'Driscoll

Acknowledgements: The Guideline Development Group would like to thank many individuals and societies who have contributed to the development of this guideline. Peer reviewers: R Beasley, P Calverley, I Coutts, C Davidson, J Gibson, B Higgins, W Macnee, W S Lim, N Linaker, M Morgan, G Nolan, M Pearson, S Prigmore, L Restrick, M Roberts, R Smith, L Ward.

The following have offered advice, either via the BTS website or directly: J Annandale, I Babin-Cooper, H Booth, R Bright-Thomas, E Buckman, K Butterfield, D Child, R Clearkin, L Cubitt, L Cutler, R Dent, M Dodd, A Duck, H Ellis, A Frew, A Groome, M Griffiths, G Hands, J Hughes, A Jones, W McConnell, S Madge, J MacMahon, H Morrison, E Myers, A Patel, G Price, A Reinhardt, R Russell, J Scullion, G Simpson, A Snelson, A Stone, C Subbe, K Swarbrick, J Tuggey, C Turnbull, C Ward, P Wills. BTS staff: S Edwards, J Moon, A Morgan, K Reid, C Routh, S Welham. Special thanks are also due to Dr Norman Johnson, Chairman of the BTS Standards of Care Committee for support and advice throughout the guideline development process.

Competing interests: No member of the Guideline Development Group is aware of any competing interests. In particular, no member of the group has any financial involvement with any company that is involved in oxygen therapy.

\section{REFERENCES}

1. Hale K, Gavin C, O'Driscoll R. An audit of oxygen use in emergency ambulances and in a hospital emergency department. Emerg Med J 2008 (in press).

2. Wilson AT, Channer KS. Hypoxaemia and supplemental oxygen therapy in the first 24 hours after myocardial infarction: the role of pulse oximetry. J $R$ Coll Physicians Lond 1997;31:657-61.

3. Cook DJ, Reeve BK, Griffith $L E$, et al. Multidisciplinary education for oxygen prescription. A continuous quality improvement study. Arch Intern Med 1996;156:1797-801

4. Small D, Duha A, Wieskopf B, et al. Uses and misuses of oxygen in hospitalized patients". Am J Med 1992;92:591-5.

5. Dodd ME, Kellet F, Davis $A$, et al. Audit of oxygen prescribing before and after the introduction of a prescription chart. BMJ 2000;321:864-5.

6. Howell M. Howell M. An audit of oxygen prescribing in acute general medical wards. Prof Nurse 2001;17:221-4.

7. Boyle M, Wong J. Prescribing oxygen therapy. An audit of oxygen prescribing practices on medical wards at North Shore Hospital, Auckland, New Zealand. NZ Med J 2006:119

8. Kbar FA, Campbell IA. Oxygen therapy in hospitalized patients: the impact of local guidelines. J Eval Clin Pract 2006;12:31-6.

9. Murphy R, Mackway-Jones K, Sammy I, et al. Emergency oxygen therapy for the breathless patient. Guidelines prepared by North West Oxygen Group. Emerg Med J 2001:18:421-3.

10. Murphy R, Driscoll P, O'Driscoll R. Emergency oxygen therapy for the COPD patient. Emerg Med J 2001;18:333-9.

11. Crapo RO, Jensen RL, Hegewald M, et al. Arterial blood gas reference values for sea level and an altitude of 1,400 meters. Am J Respir Crit Care Med 1999;160/5 Pt 1):1525-31

12. Hardie JA, Morkve 0, Ellingsen I. Effect of body position on arterial oxygen tension in the elderly. Respiration 2002;69:123-8.

13. Hardie JA, Vollmer WM, Buist AS, et al. Reference values for arterial blood gases in the elderly. Chest 2004:125:2053-60.

14. Guenard H, Marthan R. Pulmonary gas exchange in elderly subjects. Eur Respir J 1996:9:2573-7

15. Blom $\mathbf{H}$, Mulder M, Verweij W. Arterial oxygen tension and saturation in hospital patients: effect of age and activity. BMJ 1988;297:720-1.

16. Cerveri I, Zoia MC, Fanfulla F, et al. Reference values of arterial oxygen tension in the middle-aged and elderly. Am J Respir Crit Care Med 1995;152:934-41.

17. Hansen JE, Casaburi R. Patterns of dissimilarities among instrument models in measuring $\mathrm{PO}_{2}, \mathrm{PCO}_{2}$ and $\mathrm{pH}$ in blood gas laboratories. Chest 1998:113:780-7.

18. Beall CM. Oxygen saturation increases during childhood and decreases during adulthood among high altitude native Tibetans residing at 3800-4200 m. High Altitude Med Biol 2000;1:25-32.
19. Beall CM. Andean, Tibetan and Ethiopian patterns of adaptation to high-altitude hypoxia. Integr Comp Biol 2006;46:18-24.

20. Peacock AJ, Jones PL. Gas exchange at extreme altitude: results from the British 40th Anniversary Everest Expedition. Eur Respir J 1997;10:1439-44.

21. Bowton DL, Scuderi PE, Haponik EF. The incidence and effect on outcome of hypoxemia in hospitalised medical patients. Am J Med 1994;97:38-46.

22. Brain Trauma Foundation, American Association of Neurological Surgeons, Congress of Neurological Surgeons, Joint Section on Neurotrauma and Critical Care. Guidelines for the management of severe traumatic brain injury: cerebral perfusion pressure. New York: Brain Trauma Foundation, 2003:14. http://www.guideline.gov/ summary/summary.aspx?doc id $=3794 \#$ s23

23. Jubran A, Tobin MJ. Reliability of pulse oximetry in titrating supplemental oxygen therapy in ventilator-dependent patients. Chest 1990;97:1420-5.

24. Slutsky AS. Consensus conference on mechanical ventilation. Part I. European Society of Intensive Care Medicine, the ACCP and the SCCM. Intensive Care Med 1994;20:64-79

25. National Institute for Clinical Excellence (NICE). Chronic obstructive pulmonary disease. NICE Guideline CG12. 2004. http://www.nice.org.uk/guidance/CG12/ niceguidance/pdf/English.

26. Gries RE, Brooks LJ. Normal oxyhemoglobin saturation during sleep. How low does it go? Chest 1996;110:1489-92.

27. Hurtado A, Aste-Salazar H. Arterial blood gases and acid-base balance at sea level and at high altitude. J Appl Physiol 1948:1:304-25.

28. Levin KP, Hanusa BH, Rotondi A, et al. Arterial blood gas and pulse oximetry in initial management of patients with community-acquired pneumonia. J Gen Intern Med 2001;16:590-8.

29. Manley G, Knudson MM, Morabito D, et al. Hypotension, hypoxia, and head injury frequency, duration, and consequences. Arch Surg 2001;136:1118-23.

30. Moed BR, Boyd DW, Andring RE. Clinically inapparent hypoxemia after skeletal injury. The use of the pulse oximeter as a screening method. Clin Orthop 1993:293:269-73

31. Considine $\mathbf{J}$. The reliability of clinical indicators of oxygenation: a literature review. Contemp Nurs 2005;18:258-67.

32. British Thoracic Society Standards of Care Committee. Non-invasive ventilation in acute respiratory failure. Thorax 2002;57:192-211.

33. Brealey D, Singer M. Mitochondrial dysfunction in sepsis. Curr Infect Dis Rep 2003:5:365-71

34. Plant PK, Owen JL, Elliott MW. One year period prevalence study of respiratory acidosis in acute exacerbations of COPD: implications for the provision of noninvasive ventilation and oxygen administration. Thorax 2000;55:550-4.

35. Cutaia M, Rounds S. Hypoxic pulmonary vasoconstriction. Physiologic significance mechanism, and clinical relevance. Chest 1990;97:706-18.

36. Wetzel RC, Herold CJ, Zerhouni EA, et al. Hypoxic bronchodilation. J Appl Physiol 1992:73:1202-6.

37. Fidone SJ, Gonzalez C. Initiation and control of chemoreceptor activity in the carotid body. In: Cherniack NS, Widdicombe JG, eds. Handbook of physiology. Section 3: The respiratory system. Bethesda, Maryland: American Physiology Society, 1986:247-312

38. Lahiri S, Mulligan E, Nishino T, et al. Relative responses of aortic body and carotid body chemoreceptors to carboxyhemoglobinemia. J Appl Physiol 1981:50:580-6.

39. Matsuoka T, Saiki C, Mortola JP. Metabolic and ventilatory responses to anemic hypoxia in conscious rats. J Appl Physiol 1994;77:1067-72.

40. Johannes $\mathbf{T}$, Mik EG, Nohé $\mathrm{B}$, et al. Acute decrease in renal microvascular $\mathrm{PO}_{2}$ during acute normovolemic hemodilution. Am J Physiol Renal Physiol 2007:292:F796-803.

41. Rosenberger C, Mandriota S, Jürgensen JS, et al. Expression of hypoxia-inducible factor-1alpha and -2alpha in hypoxic and ischemic rat kidneys. J Am Soc Nephrol 2002:13:1721-32.

42. Eckardt KU, Bernhardt WM, Weidemann A, et al. Role of hypoxia in the pathogenesis of renal disease. Kidney Int Suppl 2005;99:S46-51.

43. Tyuma I. The Bohr effect and the Haldane effect in human hemoglobin. Jpn J Physiol 1984;34:205-16.

44. Kahler RL, Goldblatt A, Braunwald E. The effects of acute hypoxia on the systemic venous and arterial systems and on myocardial contractile force. J Clin Invest 1962;41:1553-63

45. Heistad DD, Abboud FM. Dickinson W. Richards Lecture: Circulatory adjustments to hypoxia. Circulation 1980;61:463-70.

46. Clifford PS, Hellsten Y. Vasodilatory mechanisms in contracting skeletal muscle. J Appl Physiol 2004:97:393-403.

47. Berne RM, Levy MN. Transport of oxygen and carbon dioxide: tissue oxygenation In: Physiology. 5th ed. Philadelphia: Mosby, 2005

48. Mitchell RA, et al. Respiratory responses mediated through superficial chemosensitive areas on the medulla. J Appl Physiol 1963;18:523-33.

49. Dorrington KL, Talbot NP. Human pulmonary vascular responses to hypoxia and hypercapnia. Pflugers Arch 2004;449:1-15.

50. Balanos GM, et al. Human pulmonary vascular response to $4 \mathrm{~h}$ of hypercapnia and hypocapnia measured using Doppler echocardiography. J Appl Physiol 2003:94:1543-51.

51. Lumb AB. Nunn's applied respiratory physiology. 6th ed. Oxford: Butterworth Heinemann, 2005

52. Marks B, Mitchell DG, Simelaro JP. Breath-holding in healthy and pulmonarycompromised patients: effects of hyperventilation and oxygen inspiration. J Magn Reson Imaging 1997:7:595-7. 
53. Danias PG, Stuber M, Bontar RM, et al. Navigator assessment of breath-hold duration: impact of supplemental oxygen and hyperventilation. Am J Roentgenol 1998;171:395-7.

54. McCarthy RM, Shea S, Deshpande VS, et al. Coronary MR angiography: true FISP imaging improved by prolonging breath holds with preoxygenation in healthy volunteers. Radiology 2003;227:283-8

55. Hebert PC, Wells G, Blajchman MA, et al. A multicenter, randomized, controlled clinical trial of transfusion requirements in critical care. Transfusion Requirements in Critical Care Investigators, Canadian Critical Care Trials Group. N Engl J Med 1999;340:409-17.

56. Bishop MH, Shoemaker WC, Appel PL, et al. Prospective, randomised trial of survivor values of cardiac index, oxygen delivery, and oxygen consumption as resuscitation endpoints in severe trauma. J Trauma 1995;38:780-7.

57. Velmahos GC, Demetriades D, Shoemaker WC, et al. Endpoints of resuscitation of critically injured patients: normal or supranormal? A prospective randomized trial. Ann Surg 2000;232:409-18.

58. Kern JW, Shoemaker WC. Meta-analysis of hemodynamic optimization in high-risk patients. Crit Care Med 2002;30:1686-92.

59. Boyd 0. Optimisation of oxygenation and tissue perfusion in surgical patients. Intensive Crit Care Nurs 2003;19:171-81.

60. Severinghaus JW. Simple, accurate equations for human blood $\mathrm{O}_{2}$ dissociation computations. J Appl Physiol 1979; 46:599-602.

61. Hoffman CE, Clark RT, Brown EB. Blood oxygen saturations and duration of consciousness in anoxia at high altitudes. Am J Physiol 1946;145:685-92.

62. Comroe JH, Bahnson ER, Coates EO. Mental changes occurring in chronically anoxaemic patients during oxygen therapy. JAMA 1950;143:1044-8.

63. Boycott AE, Haldane JS. The effects of low atmospheric pressure on respiration. J Physiol (Lond) 1908;37:355-77.

64. Yoneda I, Tomoda M, Tokumaru 0, et al. Time of useful consciousness determination in aircrew members with reference to prior altitude chamber experience and age. Aviat Space Environ Med 2000;71:72-6.

65. Warrel DA, Edwards RHT, Godfrey S, et al. Effect of controlled oxygen therapy on arterial blood gases in acute respiratory failure. BMJ 1970;2:452-5.

66. Campbell EJM. The management of acute respiratory failure in chronic bronchitis and emphysema. Am Rev Respir Dis 1967;96:26-639.

67. Henrion J, Minette P, Colin L, et al. Hypoxic hepatitis caused by acute exacerbation of chronic respiratory failure: a case-controlled, hemodynamic study of 17 consecutive cases. Hepatology 1999;29:427-33.

68. Henrion J, Schapira M, Luwaert R, et al. Hypoxic hepatitis: clinical and hemodynamic study in 142 consecutive cases. Medicine (Baltimore) 2003;82:392406.

69. Akero A, Christensen CC, Edvardsen A, et al. Hypoxaemia in chronic obstructive pulmonary disease patients during a commercial flight. Eur Respir J 2005;25:72530.

70. Humphreys S, Deyermond R, Bali I, et al. The effect of high altitude commercial air travel on oxygen saturation. Anaesthesia 2005;60:458-60

71. Cottrell JJ, Lebovitz BL, Fennell RG, et al. Inflight arterial saturation: continuous monitoring by pulse oximetry. Aviat Space Environ Med 1995;66:126-30.

72. Quarterman CP, Thomas AN, McKenna M, et al. Use of a patient information system to audit the introduction of modified early warning scoring. J Eval Clin Pract 2005;11:133-8.

73. Subbe $\mathbf{C P}$, Kruger $\mathrm{M}$, Rutherford $\mathrm{P}$, et al. Validation of a modified Early Warning Score in medical admissions. $0 \mathrm{~J}$ Med 2001;94:521-6.

74. Northfield TC. Oxygen therapy for spontaneous pneumothorax. BMJ 1971;4:86-8.

75. British Thoracic Society. Guidelines for the management of pleural disease. 2003. http://www.brit-thoracic.org.uk/c2/uploads/PleuralDiseaseSpontaneous.pdf

76. Tan A, Schulze A, O'Donnell CPF, et al. Air versus oxygen for resuscitation of infants at birth. Cochrane Database Syst Rev 2005;(2):CD002273.

77. Kuisma M, Boyd J, Voipio V, et al. Comparison of 30 and the $100 \%$ inspired oxygen concentrations during early post-resuscitation period: a randomised controlled pilot study. Resuscitation 2006;69:199-206.

78. Grubb NR, Simpson C, Sherwood RA, et al. Prediction of cognitive dysfunction after resuscitation from out-of-hospital cardiac arrest using serum neuron-specific enolase and protein S-100. Heart 2007;93:1268-73.

79. Greif $\mathbf{R}$, Akca O, Horn EP, et al. Supplemental perioperative oxygen to reduce the incidence of surgical-wound infection. Outcomes Research Group. N Engl J Med 2000;342:161-7.

80. Belda FJ, Aguilera L, Garcia de la Asuncion J, et al for the Spanish Reduccion de la Tasa de Infeccion Quirurgica Group. Supplemental perioperative oxygen and the risk of surgical wound infection: a randomized controlled trial. JAMA 2005;294:203542.

81. Pryor KO, Fahey TJ 3rd, Lien CA, et al. Surgical site infection and the routine use of perioperative hyperoxia in a general surgical population: a randomized controlled trial. JAMA 2004;291:79-87.

82. Attard JA, Raval MJ, Martin GR, et al. The effects of systemic hypoxia on colon anastomotic healing: an animal model. Dis Colon Rectum 2005;48:1460-70.

83. Garcia-Botello SA, Garcia-Granero E, Lillo R, et al. Randomized clinical trial to evaluate the effects of perioperative supplemental oxygen administration on the colorectal anastomosis. Br J Surg 2006;93:698-706.

84. Kranke P, Bennett M, Roeckl-Wiedmann I, et al. Hyperbaric oxygen therapy for chronic wounds. Cochrane Database Syst Rev 2004;(2):CD004123.

85. Whitney JD, Heiner S, Mygrant Bl, et al. Tissue and wound healing effects of short duration postoperative oxygen therapy. Biol Res Nurs 2001;2:206-15.
86. May A, Leone M, Afra J, et al, EFNS Task Force. EFNS guidelines on the treatment of cluster headache and other trigeminal-autonomic cephalalgias. Eur J Neurol 2006;13:1066-77.

87. Fogan L. Treatment of cluster headache. A double-blind comparison of oxygen $v$ air inhalation. Arch Neurol 1985;42:362-3.

88. Kober A, Fleischackl R, Scheck T, et al. A randomized controlled trial of oxygen for reducing nausea and vomiting during emergency transport of patients older than 60 years with minor trauma. Mayo Clinic Proc 2002;77:35-8.

89. Smith E. Oxygen for reducing nausea and vomiting during emergency ambulance transportation: a systematic review of randomised controlled trials. J Emerg Primary Health Care 2003;1:10.

90. Turan A, Apfel CC, Kumpch M, et al. Does the efficacy of supplemental oxygen for the prevention of postoperative nausea and vomiting depend on the measured outcome, observational period or site of surgery? Anaesthesia 2006;61:628-33.

91. Greif R, Laciny S, Rapf B, et al. Supplemental oxygen redices the incidence of postoperative nausea and vomiting. Anaesthesiology 1999;921:1246-52.

92. Ziavra NV, Yen Pick Sang FD, Golding JF, et al. Effect of breathing supplemental oxygen on motion sickness in healthy adults. Mayo Clinic Proc 2003;78:574-8.

93. Downs JB. Has oxygen administration delayed appropriate respiratory care? Fallacies regarding oxygen therapy. Respir Care 2003;48:611-20.

94. Lopez-Majano V, Dutton RE. Regulation of respiration during oxygen breathing in COLD. Am Rev Respir Dis 1973;108:232-40.

95. Massaro DJ, Katz S, Luchsinger PC. Effect of various modes of oxygen administration on the arterial gas values in patients with respiratory acidosis. BMJ 1962;2:627-9.

96. Thomson AJ, Webb DJ, Maxwell SR, et al. Oxygen therapy in acute medical care. BMJ 2002;324:1406-7.

97. Beasley R, Adlington S, Robinson G. Is it time to change the approach to oxygen therapy in the breathless patient. Thorax 2007;62:840-1

98. Berry RB, Mahutte CK, Kirsch JL, et al. Does the hypoxic ventilatory response predict the oxygen-induced falls in ventilation in COPD? Chest 1993;103:820-4.

99. Dick CR, Liu Z, Sassoon CS, et al. $\mathrm{O}_{2}$-induced change in ventilation and ventilatory drive in COPD. Am J Respir Crit Care Med 1997;155:609-14.

100. Erbland ML, Ebert RV, Snow SL. Interaction of hypoxia and hypercapnia on respiratory drive in patients with COPD. Chest 1990;97:1289-94.

101. Castaing Y, Manier G, Guénard H. Effect of $26 \%$ oxygen breathing on ventilation and perfusion distribution in patients with cold. Bull Eur Physiopathol Respir 1985;21:17-23

102. Aubier M, Murciano D, Milic Emili J, et al. Effects of the administration of $\mathrm{O}_{2}$ on ventilation and blood gases in patients with chronic obstructive pulmonary disease during acute respiratory failure. Am Rev Respir Dis 1980;122:747-54.

103. Pain MCF, Read DJC, Read J. Changes of arterial carbon-dioxide tension in patients with chronic lung disease breathing oxygen. Aust Ann Med 1965;14:195.

104. Robinson TD, Freiberg DB, Regnis JA, et al. The role of hypoventilation and ventilation-perfusion redistribution in oxygen-induced hypercapnia during acute exacerbations of chronic obstructive pulmonary disease. Am J Respir Crit Care Med 2000;161:1524-9.

105. Feller-Kopman D, Schwartzstein R. The role of hypoventilation and ventilationperfusion redistribution in oxygen-induced hypercapnia during acute exacerbations of chronic obstructive pulmonary disease. Am J Respir Crit Care Med 2001;163:1755.

106. Johnson JE, Peacock MD, Hayes JA, et al. Forced expiratory flow is reduced by $100 \%$ oxygen in patients with chronic obstructive pulmonary disease. South Med J 1995;88:443-9.

107. Rawles JM, Kenmure AC. Controlled trial of oxygen in uncomplicated myocardial infarction. BMJ 1976;1:1121-3.

108. Ronning $\mathbf{0 M}$, Guldvog B. Should stroke victims routinely receive supplemental oxygen? A quasi-randomized controlled trial. Stroke 1999;30:2033-7.

109. Bryan CL, Jenkinson SG. Oxygen toxicity. Clin Chest Med 1988;9:141-52.

110. Saugstad OD. Bronchopulmonary dysplasia-oxidative stress and antioxidants Semin Neonatol 2003;8:39-49.

111. Cannon RO 3rd. Mechanisms, management and future directions for reperfusion injury after acute myocardial infarction. Nat Clin Pract Cardiovasc Med 2005;2:8894.

112. Fu ES, Downs JB, Schweiger JW, et al. Supplemental oxygen impairs detection of hypoventilation by pulse oximetry. Chest 2004;126:1552-8.

113. Doelman CJ, Bast A. Oxygen radicals in lung pathology. Free Radic Biol Med 1990;9:381-400

114. Smith LL. Mechanism of paraquat toxicity in lung and its relevance to treatment Hum Toxicol 1987;6:31-6.

115. Cersosimo RJ, Matthews SJ, Hong WK. Bleomycin pneumonitis potentiated by oxygen administration. Drug Intell Clin Pharm 1985;19:921-3.

116. Nader-Djalal N, Knight PR 3rd, Thusu K, et al. Reactive oxygen species contribute to oxygen-related lung injury after acid aspiration. Anesth Analg 1998;87:127-33.

117. Knight PR, Kurek C, Davidson BA, et al. Acid aspiration increases sensitivity to increased ambient oxygen concentrations. Am J Physiol Lung Cell Mol Physiol 2000;278:L1240-7.

118. Refsum HE. Relationship between state of consciousness and arterial hypoxaemia and hypercapnia in patients with pulmonary insufficiency, breathing air. Clin Sci 1963;25:361-7.

119. Seevers MH. The narcotic properties of carbon dioxide. NY State J Med 1944:44:597-602. 
120. Dripps RD, Comroe $\mathbf{J H}$. The respiratory and circulatory response of normal man to inhalation of 7.6 and 10.4 per cent $\mathrm{CO}_{2}$. Am J Physiol 1947;149:43-51.

121. Sieker HO, Hickam JB.Carbon dioxide intoxication. Medicine (Baltimore) 1956:35:389-423.

122. Westlake EK, Simpson T, Kaye M. Carbon dioxide narcosis in emphysema. Q J Med 1955:94:155-73.

123. Barer GR, Howard P, McCurrie JR, et al. The effect of carbon dioxide and changes in blood pH on pulmonary vascular resistance in cats. Clin Sci 1967;32:361-76.

124. Kiely DG, Cargill Rl, Lipworth BJ, et al. Effects of hypercapnia on hemodynamic, inotropic, lusitropic, and electrophysiologic indices in humans. Chest 1996;109:1215-21.

125. Loeppky JA, Scotto P, Riedel CE, et al. Effects of acid-base status on acute hypoxic pulmonary vasoconstriction and gas exchange. J Appl Physiol 1992;72:1787-97.

126. Domino KB, Swenson ER, Hlastala MP, et al. Hypocapnia-induced ventilation/ perfusion mismatch: a direct $\mathrm{CO}_{2}$ or pH-mediated effect? Am J Respir Crit Care Med 1995;152(5 Pt 1):1534-9.

127. Cullen DJ, Eger El. Cardiovascular effects of carbon dioxide in man. Anesthesiology 1974; 41:345-9.

128. Goldstein B, Shannon DC, Todres ID, et al. Supercarbia in children: clinical course and outcome. Crit Care Med 1990;18:166-8.

129. O'Neill B, Mahon JM, Bradley J. Short-burst oxygen therapy in chronic obstructive pulmonary disease. Respir Med 2006;100:1129-38.

130. Philip J, Gold M, Milner A, et al. A randomized, double-blind, crossover trial of the effect of oxygen on dyspnea in patients with advanced cancer. J Pain Symptom Manage 2006;32:541-50.

131. Clemens KE, Klaschik E. Symptomatic treatment of dyspnoea in patients receiving palliative care nasal delivery of oxygen compared with opioid administration. Dtsch Med Wochenschr 2007:132:1939-43.

132. Clemens KE, Klaschik E. Symptomatic therapy of dyspnea with strong opioids and its effect on ventilation in palliative care patients. J Pain Symptom Manage 2007;33:473-81.

133. Gattinoni L, Chiumello D. Therapeutic approach to respiratory failure. In: Webb AR, Shapiro MJ, Singer M, Suter PM, eds. Oxford textbook of critical care. New York: Oxford University Press, 1999:74-8.

134. Gallagher $\mathbf{R}$, Roberts D. A systematic review of oxygen and airflow effect on relief of dyspnea at rest in patients with advanced disease of any cause. J Pain Palliat Care Pharmacother 2004;18:3-15.

135. Rowat AM, Wardlaw JM, Dennis MS, et al. Patient positioning influences oxygen saturation in the acute phase of stroke. Cerebrovasc Dis 2001;12:66-72.

136. Tyson SF, Nightingale P. The effects of position on oxygen saturation in acute stroke: a systematic review. Clin Rehabil 2004;18:863-71.

137. Bourke SJ, Munro NC, White JE, et al. Platypnoea-orthodeoxia in cryptogenic fibrosing alveolitis. Thorax 1995;89:387-9.

138. National Institute for Clinical Health and Excellence (NICE). Acutely ill patients in hospital. http://guidance.nice.org.uk/CG50.

139. Bota GW, Rowe BH. Continuous monitoring of oxygen saturation in prehospital patients with severe illness: the problem of unrecognized hypoxemia. J Emerg Med 1995:13:305-11.

140. Brown LH, Manring EA, Kornegay HB, et al. Can prehospital personnel detect hypoxemia without the aid of pulse oximeters? Am J Emerg Med 1996:14:43-4.

141. Lambert MA, Crinnion J. The role of pulse oximetry in the accident and emergency department. Arch Emerg Med 1989;6:211-5.

142. Goodacre S, Turner J, Nicholl J. Prediction of mortality amongst emergency medical admissions. Emerg Med J 2006;23:372-375.

143. Thrush DN, Downs JB, Hodges M, et al. Does significant arterial hypoxemia alter vital signs? J Clin Anesth 1997:9:355-7.

144. Howes DW, Field B, Leary T, et al. Justification of pulse oximeter costs for paramedic prehospital providers. Prehosp Emerg Care 2000;4:151-5.

145. Macnab AJ, Susak L, Gagnon FA, et al. The cost-benefit of pulse-oximeter use in the prehospital environment. Prehosp Disaster Med 1999;14:245-50.

146. Jensen LA, Onyskiw JE, Prasad NG. Meta-analysis of arterial oxygen saturation monitoring by pulse oximetry in adults. Heart Lung 1998;27:387-408

147. Aughey K, Hess D, Eitel D, et al. An evaluation of pulse oximetry in prehospital care. Ann Emerg Med 1991;20:887-91.

148. Modica R, Rizzo A. Accuracy and response time of a portable pulse oximeter. The Pulsox-7 with a finger probe. Respiration 1991:58:155-7.

149. Severinghaus JW, Naifeh $\mathrm{KH}$. Accuracy of response of six pulse oximeters to profound hypoxia. Anesthesiology 1987;67:551-8.

150. Severinghaus JW, Naifeh $\mathrm{KH}$, Koh SO. Errors in 14 pulse oximeters during profound hypoxia. J Clin Monit 1989;5:72-81.

151. Kelly AM, McAlpine R, Kyle E. How accurate are pulse oximeters in patients with acute exacerbations of chronic obstructive airways disease? Respir Med 2001;95:336-40.

152. Perkins GD, McAuley DF, Giles $S$, et al. Do changes in pulse oximeter oxygen saturation predict equivalent changes in arterial oxygen saturation? Crit Care 2003:7:R67-71

153. Pedersen T, Dyrlund Pedersen B, Moller AM. Pulse oximetry for perioperative monitoring. Cochrane Database Syst Rev 2003;(3):CD002013.

154. Falconer RJ, Robinson BJ. Comparison of pulse oximeters: accuracy at low arterial pressure in volunteers. Br J Anaesth 1990;65:552-7.

155. Lee WW, Mayberry K, Crapo R, et al. The accuracy of pulse oximetry in the emergency department. Am J Emerg Med 2000;18:427-31.
156. Bickler PE, Feiner JR, Severinghaus JW. Effects of skin pigmentation on pulse oximeter accuracy at low saturation. Anesthesiology 2005;102:715-9.

157. Lee KH, Hui KP, Tan WC, et al. Factors influencing pulse oximetry as compared to functional arterial saturation in multi-ethnic Singapore. Singapore Med J 1993; 34:385-7.

158. Zeballos RJ, Weisman IM. Reliability of noninvasive oximetry in black subjects during exercise and hypoxia. Am Rev Respir Dis 1991:144:1240-4.

159. Comber JT, Lopez BL. Evaluation of pulse oximetry in sickle cell anemia patients presenting to the emergency department in acute vasoocclusive crisis. Am J Emerg Med 1996:14:16-8.

160. Ortiz F0, Aldrich TK, Nagel RL, et al. Accuracy of pulse oximetry in sickle cell disease. Am J Respir Crit Care Med 1999:159:447-51.

161. Barker SJ. "Motion-resistant" pulse oximetry: a comparison of new and old models. Anesth Analg 2002;95:967-72.

162. Gehring $\mathbf{H}$, Hornberger $\mathrm{C}$, Matz $\mathrm{H}$, et al. The effects of motion artifact and low perfusion on the performance of a new generation of pulse oximeters in volunteers undergoing hypoxemia. Respir Care 2002;47:48-60.

163. Barker SJ, Hyatt J, Shah NK, et al. The effect of sensor malpositioning on pulse oximeter accuracy during hypoxemia. Anesthesiology 1993;79:248-54.

164. Hamber EA, Bailey PL, James SW, et al. Delays in the detection of hypoxemia due to site of pulse oximetry probe placement. J Clin Anesth 1999;11:113-8.

165. Tittle M, Flynn MB. Correlation of pulse oximetry and co-oximetry. Dimens Crit Care Nurs 1997:16:88-95.

166. Eaton T, Rudkin S, Garrett JE. The clinical utility of arterialized earlobe capillary blood in the assessment of patients for long-term oxygen therapy. Respir Med 2001:95:655-60.

167. Murphy R, Thethy S, Raby S, et al. Capillary blood gases in acute exacerbations of COPD. Respir Med 2006;100:682-6.

168. Zavorsky GS, Cao J, Mayo NE, et al. Arterial versus capillary blood gases: a metaanalysis. Respir Physiol Neurobiol 2007;155:268-79.

169. Wimpress S, Vara DD, Brightling CE. Improving the sampling technique of arterialized capillary samples to obtain more accurate $\mathrm{PaO}_{2}$ measurements. Chron Respir Dis 2005;2:47-50.

170. Pitkin AD, Roberts CM, Wedzicha JA. Arterialised earlobe blood gas analysis: an underused technique. Thorax 1994;49:364-6.

171. Dar K, Williams T, Aitken R, et al. Arterial versus capillary sampling for analysing blood gas pressures. BMJ 1995;310:24-5.

172. Lightowler JVJ, Elliott MW. Local anaesthetic infiltration prior to arterial puncture a survey of current practice and a randomised double blind placebo controlled trial. J R Coll Physicians Lond 1997:31:645-6.

173. Valentine RJ, Modrall JG, Clagett GP. Hand ischemia after radial artery cannulation. J Am Coll Surg 2005:201:18-22.

174. Tatevossian RG, Wo CC, Velmahos GC, et al. Transcutaneous oxygen and $\mathrm{CO}_{2}$ as early warning of tissue hypoxia and hemodynamic shock in critically ill emergency patients. Crit Care Med 2000;28:2248-53.

175. Slinger $\mathbf{P}$, Blundell PE, Metcalf IR, et al. Management of massive grain aspiration. Anesthesiology 1997;87:993-5.

176. Cham GW, Tan WP, Earnest A, et al. Clinical predictors of acute respiratory acidosis during exacerbation of asthma and chronic obstructive pulmonary disease. Eur J Emerg Med 2002:9:225-32.

177. Kelly AM, Kyle E, McAlpine R. Venous pCO(2) and $\mathrm{pH}$ can be used to screen for significant hypercarbia in emergency patients with acute respiratory disease. J Emerg Med 2002;22:15-9.

178. Malatesha G, Singh NK, Bharija A, et al. Comparison of arterial and venous $\mathrm{pH}$, bicarbonate, $\mathrm{PCO}_{2}$ and $\mathrm{PO}_{2}$ in initial emergency department assessment. Emerg Med J 2007:24:569-71.

179. Rang LC, Murray HE, Wells GA, et al. Can peripheral venous blood gases replace arterial blood gases in emergency department patients? CJEM 2002;4:7-15.

180. Levine RL, Wayne MA, Miller CC. End-tidal carbon dioxide and outcome of out-ofhospital cardiac arrest. N Engl J Med 1997;337:301-6.

181. Grmec S, Klemen P. Does the end-tidal carbon dioxide $\left(\mathrm{EtCO}_{2}\right)$ concentration have prognostic value during out-of-hospital cardiac arrest? Eur $\mathrm{J}$ Emerg Med 2001:8:263-9.

182. Ahrens T, Schallom $\mathrm{L}$, Bettorf $\mathrm{K}$, et al. End-tidal carbon dioxide measurements as a prognostic indicator of outcome in cardiac arrest. Am J Crit Care 2001;10:391-8.

183. Isserles SA, Breen PH. Can changes in end-tidal $\mathrm{PCO}_{2}$ measure changes in cardiac output? Anesth Analg 1991;73:808-14.

184. Kocher S, Rohling R, Tschupp A. Performance of a digital $\mathrm{PaCO}_{2} / \mathrm{SPO}_{2}$ ear sensor J Clin Monit Comput 2004:18:75-9.

185. Parker SM, Gibson GJ. Evaluation of a transcutaneous carbon dioxide monitor ("TOSCA") in adult patients in routine respiratory practice. Respir Med 2007:101:261-4.

186. Raffin TA. Indications for arterial blood gas analysis. Ann Intern Med 1986;105:390-8.

187. British Thoracic Society. British guideline on the management of asthma. 2008 http://www.brit-thoracic.org.uk/Portals/0/Clinical\%20Information/Asthma/ Guidelines/asthma final2008.pdf.

188. Waldau T, Larsen VH, Bonde J. Evaluation of five oxygen delivery devices in spontaneously breathing subjects by oxygraphy. Anaesthesia 1998;53:256-63.

189. Resuscitation Council UK. Guideline for Adult Advance Life Support. 2005. http:// www.resus.org.uk/pages/als.pdf.

190. Forst H. Maximising oxygen delivery in critically ill patients: a rational therapeutic approach (in German). Anaesthesist 1997:46:46-52. 
191. Gueugniaud PY, Bertin Maghit M, Hirschauer C, et al. In the early stage of major burns, is there a correlation between survival, interleukin-6 levels and oxygen delivery and consumption? Burns 1997;23:426-31.

192. Holm C, Melcer B, Horbrand F, et al. The relationship between oxygen delivery and oxygen consumption during fluid resuscitation of burn-related shock. J Burn Care Rehabil 2000;21:147-54.

193. Steltzer H, Hiesmayr M, Mayer N, et al. The relationship between oxygen delivery and uptake in the critically ill: is there a critical or optimal therapeutic value? A metaanalysis. Anaesthesia 1994;49:229-36.

194. Dellinger RP, Carlet JM, Masur H, et al, Surviving Sepsis Campaign Management Guidelines Committee. Surviving Sepsis Campaign guidelines for management of severe sepsis and septic shock. Crit Care Med 2004;32:858-73.

195. Reinhart K, Bloos F, Konig F, et al. Reversible decrease of oxygen consumption by hyperoxia. Chest 1991;99:690-4.

196. Rossi P, Tauzin L, Weiss M, et al. Could hyperoxic ventilation impair oxygen delivery in septic patients? Clin Physiol Funct Imaging 2007;27:180-4.

197. Mildh LH, Piilonen A, Kirvela OA. Supplemental oxygen is not required in trauma patients treated with IV opiates. Am J Emerg Med 2003;21:35-8.

198. Price GF, Goodwin NM. Hypoxaemia and leucopenia after fractures. Injury 1980;12:115-8.

199. Wong MW, Tsui HF, Yung SH, et al. Continuous pulse oximeter monitoring for inapparent hypoxemia after long bone fractures. J Trauma 2004;56:356-62.

200. Gentleman D. Causes and effects of systemic complications among severely head injured patients transferred to a neurosurgical unit. Int Surg 1992;77:297-302.

201. Pfenninger EG, Lindner KH. Arterial blood gases in patients with acute head injury at the accident site and upon hospital admission. Acta Anaesthesiol Scand 1991;35:148-52.

202. Robertson C. Desaturation episodes after severe head injury: influence on outcome. Acta Neurochir Suppl (Wien) 1993;59:98-101.

203. Adamides AA, Winter CD, Lewis PM, et al. Current controversies in the management of patients with severe traumatic brain injury. Aust NZ J Surg 2006;76:163-74.

204. Magnoni S, Ghisoni L, Locatelli M, et al. Lack of improvement in cerebral metabolism after hyperoxia in severe head injury: a microdialysis study. J Neurosurg 2003;98:952-8.

205. Longhi L, Stoccehetti N. Hyperoxia in head injury: therapeutic tool? Curr Opin Crit Care 2004;10:105-9.

206. Diringer MN, Aiyagari V, Zazulia AR, et al. Effect of hyperoxia on cerebral metabolic rate for oxygen measured using positron emission tomography in patients with acute severe head injury. J Neurosurg 2007;106:526-9.

207. Kao LW, Nanagas KA. Toxicity associated with carbon monoxide. Clin Lab Med 2006;26:99-125.

208. Juurlink DN, Buckley NA, Stanbrook MB, et al. Hyperbaric oxygen for carbon monoxide poisoning. Cochrane Database Syst Rev 2005;(1):CD002041.

209. Weaver LK, Valentine KJ, Hopkins RO. Carbon monoxide poisoning: risk factors for cognitive sequelae and the role of hyperbaric oxygen. Am J Respir Crit Care Med 2007; 176:491-7.

210. Ford DJ, Rothwell RP. 'Safe oxygen' in acute asthma: prospective trial using 35\% Ventimask prior to admission. Respir Med 1989;83:189-94.

211. Rodrigo GJ, Rodriguez Verde M, Peregalli V, et al. Effects of short-term $28 \%$ and $100 \%$ oxygen on $\mathrm{PaCO}_{2}$ and peak expiratory flow rate in acute asthma: a randomized trial. Chest 2003;124:1312-7.

212. Chien JW, Ciufo R, Novak R, et al. Uncontrolled oxygen administration and respiratory failure in acute asthma. Chest 2000;117:728-33.

213. British Thoracic Society. Guideline for pneumonia. http://www.brit-thoracic.org. uk/bts_guidelines_pneumonia_html.

214. Dudgeon DJ, Lertzman M. Dyspnea in the advanced cancer patient. J Pain Symptom Manage 1998;16:212-9.

215. Ripamonti C. Management of dyspnea in advanced cancer patients. Supportive Care in Cancer 1999;7:233-43.

216. de Bruera $\mathbf{E}$, Stoutz N, Velasco-Leiva A, et al. Effects of oxygen on dyspnoea in hypoxaemic terminal-cancer patients. Lancet 1993;342:13-4.

217. Booth S, Kelly MJ, Cox NP, et al. Does oxygen help dyspnoea in patients with cancer? Am J Respir Crit Care Med 1996;153:1515-8.

218. Navigante AH, Cerchietti LCA, Cabalar ME. Morphine plus midazolam versus oxygen therapy on severe dyspnea management in the last week of life in hipoxemic advanced cancer patients. Medicina Paliativa 2003;10:14-9.

219. Booth S, Wade R, Johnson M, et al. The use of oxygen in the palliation of breathlessness. A report of the expert working party of the Scientific Committee of the Association of Palliative Medicine. Respir Med 2004;98:66-77.

220. Jardin F, Gurdjian F, Desfonds $P$, et al. Hemodynamic factors influencing arterial hypoxemia in massive pulmonary embolism with circulatory failure. Circulation 1979;59:909-12.

221. Creamer JE, Edwards JD, Nightingale P. Hemodynamic and oxygen transport variables in cardiogenic shock secondary to acute myocardial infarction, and response to treatment. Am J Cardiol 1990;65:1297-300.

222. Aubier M, Trippenbach T, Roussos C. Respiratory muscle fatigue during cardiogenic shock and prolongs survival, which may bear important therapeutic implications. J Appl Physiol 1981;51:499-508.

223. Swedberg K, Cleland J, Dargie $\mathrm{H}$, et al, Task Force for the Diagnosis and Treatment of Chronic Heart Failure of the European Society of Cardiology. Guidelines for the diagnosis and treatment of chronic heart failure: executive summary (update 2005). Eur Heart J 2005;26:1115-40.
224. Vaisanen IT, Rasanen J. Continuous positive airway pressure and supplemental oxygen in the treatment of cardiogenic pulmonary edema. Chest 1987;92:481-5.

225. Kallio T, Kuisma M, Alaspaa A, et al. The use of prehospital continuous positive airway pressure treatment in presumed acute severe pulmonary edema. Prehosp Emerg Care 2003; 7:209-13

226. Nadar S, Prasad N, Taylor RS, et al. Positive pressure ventilation in the management of acute and chronic cardiac failure: a systematic review and metaanalysis. Int J Cardiol 2005;99:171-85.

227. Masip J, Roque M, Sánchez B, et al. Noninvasive ventilation in acute cardiogenic pulmonary edema: systematic review and meta-analysis. JAMA 2005;294:312430.

228. Moller JT, Johannessen NW, Espersen K, et al. Randomized evaluation of pulse oximetry in 20,802 patients: II. Perioperative events and postoperative complications. Anesthesiology 1993;78:445-53.

229. Scottish Intercollegiate Guideline Network (SIGN). Postoperative management in adults. Guideline No 77. 2004. http://www.sign.ac.uk/pdf/sign77.pdf.

230. Strachan L, Noble DW. Hypoxia and surgical patients-prevention and treatment of an unnecessary cause of morbidity and mortality. J R Coll Surg Edinb 2001;46:297302.

231. Robieux IC, Kellner KD, Coppes MJ. Analgesia in children with sickle cell crisis; comparison of intermittent opioids vs continuous infusion of morphine and placebo controlled study of oxygen inhalation. Paediatr Haematol Oncol 1992;9:317-26.

232. Zipursky A, Robieux IC, Brown EJ, et al. Oxygen therapy in sickle cell disease. Am J Pediatr Haematol Oncol 1992;14:222-8.

233. Homi J, Levee L, Higgs D, et al. Pulse oximetry in a cohort study of sickle disease Clinic Lab Haematol 1997;19:17-22.

234. Rees DC, Olujohungbe AD, Parker N, et al. Guidelines for the management of the acute painful crisis in sickle cell disease. Br J Haematol 2003;120:744-52.

235. Joosten SA, Koh MS, Bu X, et al. The effects of oxygen therapy in patients presenting to an emergency department with exacerbation of chronic obstructive pulmonary disease. Med J Aust 2007;186:235-8.

236. Denniston AK, O'Brien C, Stableforth D. The use of oxygen in acute exacerbations of chronic obstructive pulmonary disease: a prospective audit of pre-hospital and hospital emergency management. Clin Med 2002;2:449-51.

237. Jeffrey AA, Warren PM, Flenley DC. Acute hypercapnic respiratory failure in patients with chronic obstructive lung disease: risk factors and use of guidelines for management. Thorax 1992;47:34-40.

238. Gomersall CD, Joynt GM, Freebairn RC, et al. Oxygen therapy for hypercapnic patients with chronic obstructive pulmonary disease and acute respiratory failure: a randomized, controlled pilot study. Crit Care Med 2002;30:113-6.

239. Esteban A, Cerda E, De La Cal MA, et al. Hemodynamic effects of oxygen therapy in patients with acute exacerbations of chronic obstructive pulmonary disease. Chest 1993;104:471-5.

240. Schumaker GL, Epstein SK. Managing acute respiratory failure during exacerbation of chronic obstructive pulmonary disease. Respir Care 2004;49:766-82.

241. Warren PM, Flenley DC, Millar JS, et al. Respiratory failure revisited: acute exacerbations of chronic bronchitis between 1961-68 and 1970-76. Lancet 1980;1:467-70.

242. Hutchison DCS, Flenley DC, Donald KW. Controlled oxygen therapy in respiratory failure. BMJ 1964;2:1159-66.

243. Mithoefer JC, Karetsky MS, Mead GD. Oxygen therapy in respiratory failure. N Engl J Med 1967;277:947-9.

244. Rudolf M, Turner JAMcM, Harrison BDW, et al. Changes in arterial blood gases during and after a period of oxygen breathing in patients with chronic hypercapnic respiratory failure and in patients with asthma. Clin Sci 1979;57:389-96.

245. Schiff MM, Massaro D. Effect of oxygen administration by a Venturi apparatus on arterial blood gas values in patients with respiratory failure. $N$ Engl J Med 1967:277:950-3

246. King TKC, Ali N, Briscoe WA. Treatment of hypoxia with 24 per cent oxygen. Am Rev Respir Dis 1973;108:19-29.

247. Rudolf $\mathbf{M}$, Banks RA, Semple SJ. Hypercapnia during oxygen therapy in acute exacerbations of chronic respiratory failure. Lancet 1977;2:483-6.

248. McNichol MW, Campbell EJM. Severity of respiratory failure. Lancet 1965;1:3368.

249. Smith JP, Stone RW, Muschenheim C. Acute respiratory failure in chronic lung disease. Am Rev Respir Dis 1968;97:791-803.

250. Campbell EJM. The relation between oxygen concentrations of inspired air and arterial blood. Lancet 1960;2:10-11.

251. Campbell EJM. A method of controlled oxygen administration which reduces the risk of carbon-dioxide retention. Lancet 1960;2:12-4

252. Lal S. Blood gases in respiratory failure. Lancet 1965;1:339-41.

253. Madden BP, Kariyawasam H, Siddiqi AJ, et al. Noninvasive ventilation in cystic fibrosis patients with acute or chronic respiratory failure. Eur Respir J 2002;19:310 3.

254. Jones HA, Turner SL, Hughes JM. Performance of the large-reservoir oxygen mask (Ventimask). Lancet 1984;1:427-31.

255. Quint JK, Ward L, Davison AG. Previously undiagnosed obesity hypoventilation syndrome. Thorax 2007:62:402-3.

256. McNulty PH, King N, Scott S, et al. Effects of supplemental oxygen administration on coronary blood flow in patients undergoing cardiac catheterization. Am J Physiol Heart Circ Physiol 2005;288:H1057-62. 
257. Harten JM, Anderson KJ, Kinsella J, et al. Normobaric hyperoxia reduces cardiac index in patients after coronary artery bypass surgery. J Cardiothorac Vasc Anesth 2005;19:173-5.

258. Frobert $\mathbf{0}$, Moesgaard J, Toft E, et al. Influence of oxygen tension on myocardial performance. Evaluation by tissue Doppler imaging. Cardiovasc Ultrasound 2004;2:22.

259. Maroko PR, Radvany P, Braunwald E, et al. Reduction of infarct size by oxygen inhalation following acute coronary occlusion. Circulation 1975;52:360-8

260. Madias JE, Hood WB. Reduction of precordial ST-segment elevation in patients with anterior myocardial infarction by oxygen breathing. Circulation 1976;53:I198200.

261. Fillmore SJ, Shapiro M, Killip T. Arterial oxygen tension in acute myocardial infarction: serial analysis of clinical state and blood gas changes. Am Heart $J$ 1970;79:620-9.

262. Waring WS, Thomson AJ, Adwani SH, et al. Cardiovascular effects of acute oxygen administration in healthy adults. J Cardiovasc Pharmacol 2003;42:245-50.

263. Kaneda T, Ku K, Inoue T, et al. Postischemic reperfusion injury can be attenuated by oxygen tension control. Jpn Circ J 2001;65:213-8.

264. Haque WA, Boehmer J, Clemson BS, et al. Hemodynamic effects of supplemental oxygen administration in congestive heart failure. J Am Coll Cardiol 1996;27:353-7.

265. McNulty PH, Robertson BJ, Tulli MA, et al. Effect of hyperoxia and vitamin $\mathrm{C}$ on coronary blood flow in patients with ischemic heart disease. J Appl Physiol 2007; 102:2040-5.

266. Nicholson C. A systematic review of the effectiveness of oxygen in reducing acute myocardial ischaemia. J Clin Nurs 2004;13:996-1007.

267. Beasley R, Aldington $S$, Weatherall $M$, et al. Oxygen therapy in myocardial infarction: an historical perspective. J $R$ Soc Med 2007:100:130-3.

268. Neill WA. The effects of arterial hypoxemia and hyperoxia on oxygen availability for myocardial metabolism: patients with and without coronary artery disease. Am J Cardiol 1969;24:166-71.

269. Lal S, Savidge RS, Chhabra GP. Oxygen administration after myocardial infarction. Lancet 1969:1:381-3.

270. Anon. The pre-hospital management of acute heart attacks. Recommendations of a Task Force of the The European Society of Cardiology and The European Resuscitation Council. Eur Heart J 1998;19:1140-64.

271. Van de Werf F, Ardissino D, Betriu A, et al, Task Force on the Management of Acute Myocardial Infarction of the European Society of Cardiology. Management of acute myocardial infarction in patients presenting with ST-segment elevation. Eur Heart J 2003;24:28-66.

272. Scottish Intercollegiate Guideline Network (SIGN). Acute coronary syndromes. A national clinical guideline. Guideline No 93. 2007. http://www.sign.ac. uk/pdf/sign93.pdf.

273. Arntz HR, Bossaert L, Filippatos GS, European Resuscitation Council. European Resuscitation Council guidelines for resuscitation 2005. Section 5. Initial management of acute coronary syndromes. Resuscitation 2005;67(Suppl 1):S8796.

274. Fisher JD, Brown N, Cooke MW. UK ambulance service clinical practice guidelines 2006. Norwich: Edexcel. http://www2.warwick.ac.uk/fac/med/research/hsri/ emergencycare/jrcalc_2006/guidelines/oxygen_oxg.pdf (accessed February 2008).

275. Royal College of Physicians. National clinical guideline for stroke. 2nd ed. 2004 http://www.strokecenter.org/prof/guidelines.htm.

276. Bamber JH, Dresner M. Aortocaval compression in pregnancy: the effect of changing the degree and direction of lateral tilt on maternal cardiac output. Anaesth Analg 2003;97:256-8

277. Fawole B, Hofmeyr GJ. Maternal oxygen administration for fetal distress. Cochrane Database Syst Rev 2003;(4):CD000136.

278. Saisch SG, Wessely S, Gardner WN. Patients with acute hyperventilation presenting to an inner-city emergency department. Chest 1996:110:952-7.

279. Steurer J, Hoffmann U, Dur $\mathrm{P}$, et al. Changes in arterial and transcutaneous oxygen and carbon dioxide tensions during and after voluntary hyperventilation. Respiration 1997:64:200-5.

280. Callaham M. Hypoxic hazards of traditional paper bag rebreathing in hyperventilating patients. Ann Emerg Med 1989;18:622-8.

281. Demeere JL. Paraquat toxicity. The use of hypoxic ventilation. Acta Anaesthesiol Belg 1984;35:219-30.

282. Austin M, Wood-Baker R. Oxygen therapy in the pre-hospital setting for acute exacerbations of chronic obstructive pulmonary disease. Cochrane Database Syst Rev 2006; (3):CD005534.

283. Durrington HJ, Flubacher M, Ramsay CF, et al. Initial oxygen management in patients with an exacerbation of chronic obstructive pulmonary disease. $0 \mathrm{~J} \mathrm{Med}$ 2005:98:499-504.

284. Gooptu B, Ward L, Ansari SO, et al. Oxygen alert cards and controlled oxygen: preventing emergency admissions at risk of hypercapnic acidosis receiving high inspired oxygen concentrations in ambulances and A\&E departments. Emerg Med J 2006;23:636-8.

285. Tooley C, Ellis D, Greggs D, et al. Too much of a good thing? Oxygen alert cards are helpful for COPD patients at risk of oxygen toxicity. Thorax 2006;61(Suppl II):112.

286. Boumphrey SM, Morris EA, Kinsella SM. 100\% inspired oxygen from a Hudson mask: a realistic goal? Resuscitation 2003:57:69-72.

287. Jensen AG, Johnson A, Sandstedt S. Rebreathing during oxygen treatment with face mask. The effect of oxygen flow rates on ventilation. Acta Anaesthesiol Scand 1991;35:289-92.
288. Milross J, Young $\mathrm{HH}$, Donnelly P. The oxygen delivery characteristics of the Hudson Oxy-one face mask. Anaesth Intensive Care 1989;17:180-4.

289. Stausholm K, Rosenberg-Adamsen S, Skriver M, et al. Comparison of three devices for oxygen administration in the late postoperative period. $\mathrm{Br} J$ Anaesth 1995;74:607-9

290. Costello RW, Liston R, McNicholas WT. Compliance at night with low flow oxygen therapy: a comparison of nasal cannulae and Venturi face masks. Thorax 1995;50:405-6.

291. Zevola DR, Maier CB. Use of nasal cannula versus face mask after extubation in patients after cardiothoracic surgery. Crit Care Nurs 2001;21:47-53.

292. Hunter J, Olson LG. Performance of the Hudson Multi-Vent oxygen mask. Med J Aust 1988:148:444-7.

293. Bone RC, Pierce AK, Johnson RL Jr. Controlled oxygen administration in acute respiratory failure in chronic obstructive pulmonary disease: a reappraisal. Am J Med 1978:65:896-902.

294. DeGaute JP, Domenighetti G, Naeije R, et al. Oxygen delivery in acute exacerbation of chronic obstructive pulmonary disease. Effects of controlled oxygen therapy. Am Rev Respir Dis 1981;124:26-30.

295. Bazuaye EA, Stone TN, Corris PA, et al. Variability of inspired oxygen concentration with nasal cannulas. Thorax 1992:47:609-11.

296. Wettstein RB, Shelledy DC, Peters Jl. Delivered oxygen concentrations using low flow and high-flow nasal cannulas. Respir Care 2005;50:604-9.

297. Agusti AG, Carrera M, Barbe F, et al. Oxygen therapy during exacerbations of chronic obstructive pulmonary disease. Eur Respir J 1999;14:934-9.

298. Williams AB, Jones PL, Mapleson WW. A comparison of oxygen therapy devices used in the postoperative recovery period. Anaesthesia 1988;43:131-5.

299. Bolton J, Russell WJ. Are nasal spectacles adequate for supplementary oxygen in patients after anaesthesia? Int J Nurs Pract 2001;7:329-35.

300. Nolan KM, Winyard JA, Goldhill DR. Comparison of nasal cannulae with face mask for oxygen administration to postoperative patients. Br J Anaesth 1993;70:440-2.

301. Health and Safety Executive. Safe use of gas cylinders. 2004. http://www.hse. gov.uk/cdg/pdf/safusgc.pdf.

302. Anon. The carriage of dangerous goods and use of transportable pressure equipment regulations 2004. Statutory Instrument 2004 No. 568. http://www.opsi. gov.uk/si/si2004/20040568.htm.

303. Khan SY, O'Driscoll BR. Is nebulized saline a placebo in COPD? BMC Pulm Med 2004:4:9.

304. Poole PJ, Brodie SM, Stewart JM, et al. The effects of nebulised isotonic saline and terbutaline on breathlessness in severe chronic obstructive pulmonary disease (COPD). Aust NZ J Med 1998;28:322-6.

305. Conway JH, Fleming JS, Perring $S$, et al. Humidification as an adjunct to chest physiotherapy in aiding tracheo-bronchial clearance in patients with bronchiectasis. Respir Med 1992;86:109-14.

306. Andres D, Thurston N, Brant R, et al. Randomized double-blind trial of the effects of humidified compared with nonhumidified low flow oxygen therapy on the symptoms of patients. Can Respir J 1997:4:76-80.

307. Campbell EJ, Baker MD, Crites-Silver P. Subjective effects of humidification of oxygen for delivery by nasal cannula. A prospective study. Chest 1988;93:289-93.

308. Cameron JL, Reese WA, Tayal VS, et al. Bacterial contamination of ambulance oxygen humidifier water reservoirs: a potential source of pulmonary infection. Ann Emerg Med 1986;15:1300-2.

309. Khoukaz G, Gross NJ. Effects of salmeterol on arterial blood gases in patients with stable chronic obstructive pulmonary disease. Comparison with albuterol and ipratropium. Am J Respir Crit Care Med 1999:160:1028-30.

310. Gunawardena KA, Patel B, Campbell IA, et al. Oxygen as a driving gas for nebulisers: safe or dangerous? BMJ (Clin Res Ed) 1984;288:272-4.

311. Hedges JR, Cionni DJ, Amsterdam JT, et al. Oxygen desaturation in adults following inhaled metaproterenol therapy. J Emerg Med 1987;5:77-81.

312. Higgins RM, Cookson WO, Chadwick GA. Changes in blood gas levels after nebuhaler and nebulizer administration of terbutaline in severe chronic airway obstruction. Bull Eur Physiopathol Respir 1987;23:261-4.

313. Royal College of Physicians of London. Domiciliary oxygen therapy services. Clinical guidelines and advice for prescribers. Report of a Working Party of the Royal College of Physicians, 1999

\section{APPENDICES}

\section{List of appendices available on the British Thoracic Society website (www.brit-thoracic.org.uk)}

Table 1 Critical illnesses requiring high levels of supplemental oxygen.

Table 2 Serious illnesses requiring moderate levels of supplemental oxygen if the patient is hypoxaemic

Table 3 Patients requiring controlled or low-dose oxygen therapy.

Table 4 Conditions for which oxygen is not required unless the patient is hypoxaemic

Chart 1 Oxygen prescription for acutely hypoxaemic patients in hospital.

Chart 2 Flow chart for oxygen administration on general wards in hospitals.

Chart 3 Working example of oxygen section for a hospital prescription chart.

Chart 4 Working example of the respiratory section of an observation chart for hospital use (all of the above are available within the guideline but indexed here for the benefit of online users). 
Appendix 1 Summary of recommendations concerning administration and monitoring of oxygen therapy.

Appendix 2 Summary of guideline for hospital use.

Appendix 3 Example of local oxygen policy.

Appendix 4 Summary of guideline and flow charts for emergency oxygen use in ambulances, community and prehospital settings (including example of oxygen alert card).

Appendix 5 Patient information sheet.

Appendix 6 Summary of prescription, administration, monitoring, weaning and discontinuation of oxygen therapy.

Appendix 7 Example of audit tool.

Appendix 8 Lecture on emergency oxygen use for doctors.

Appendix 9 Teaching aids on emergency oxygen use for nurses, midwives, pharmacists, physiotherapists and other practitioners who use oxygen.

Appendix 10 Key points for hospital managers and oxygen champions.

Appendix 11 Key points for primary care trust managers, practice-based commissioning groups and general practice managers.

Appendix 12 Key points for ambulance service managers.

Appendix 13 List of NHS Trusts with oxygen champions.

Appendix 14 Details of search methodology.

\section{Abbreviation \\ ABG \\ BTS \\ $\mathrm{CaO}_{2}$ \\ $\mathrm{CO}$ \\ $\mathrm{CO}_{2}$ \\ COPD \\ CPAP \\ $\mathrm{Do}_{2}$ \\ DPG \\ ELBG \\ EPR \\ EWS (mEWS) \\ $\mathrm{FlO}_{2}$}

$\left[\mathrm{H}^{+}\right]$

$\mathrm{Hb}$
HPV

ICU

IPPV

$\mathrm{kPa}$

MC mask

$\mathrm{mm} \mathrm{Hg}$

NICE

NIPPV

NIV

$\mathrm{O}_{2}$

$\mathrm{PCO}_{2}$

$\mathrm{PaCO}_{2}$

$\mathrm{PACO}_{2}$

$\mathrm{PO}_{2}$

$\mathrm{PaO}_{2}$

$\mathrm{PAO}_{2}$

$\mathrm{pH}$

$\mathrm{PlO}_{2}$

PRN

ROS

SD

SIGN

$\mathrm{SaO}_{2}$

$\mathrm{SpO}_{2}$

$\dot{\mathrm{V}}_{2}$

$\mathrm{V} / 0$

$\mathrm{V} / 0$ mismatch earlobe blood gases

electronic patient record

Early Warning Score System or modified EWS

fraction of inspired oxygen (eg, $21 \%$ oxygen $=\mathrm{FlO}_{2}$ 2.21)

hydrogen ion concentration (normal range $35-45 \mathrm{nmol} / \mathrm{l}(\mathrm{pH}$

7.35-7.45): lower levels are alkalotic, higher levels are acidotic)

haemoglobin (carries oxygen in the blood stream)

Symbols

$>$

$<$

$\geqslant$

$\leqslant$ hypoxic pulmonary vasoconstriction

intensive care unit

intermittent positive pressure ventilation

kilo Pascal (unit of measurement for pressures) $1 \mathrm{kPa}$

$=7.5 \mathrm{~mm} \mathrm{Hg}$

medium concentration mask (also known as simple face mask) millimetres of mercury (unit of measurement for pressures)

National Institute for Health and Clinical Excellence

non-invasive positive pressure ventilation

non-invasive ventilation

oxygen

carbon dioxide tension (partial pressure) in blood or alveolus arterial carbon dioxide tension (partial pressure): normal range is $4.6-6.1 \mathrm{kPa}(34-46 \mathrm{~mm} \mathrm{Hg})$

alveolar carbon dioxide tension

oxygen tension (partial pressure) in blood or alveolus or tissues arterial oxygen tension (normal ranges shown in table 7)

alveolar oxygen tension

unit of measurement for acidity of blood (normal range 7.35-

$7.45\left(\left[\mathrm{H}^{+}\right] 35-45 \mathrm{nmol} / \mathrm{l}\right)$ : lower levels are acidotic, higher levels are alkalotic

inspired oxygen tension

(on prescriptions) as required, as the need arises (from Latin pro re nata)

reactive oxygen species

standard deviation from the mean

Scottish Intercollegiate Guideline Network

arterial oxygen saturation

arterial oxygen saturation measured by pulse oximetry

rate of oxygen consumption by the body (normal $\sim 250 \mathrm{ml} / \mathrm{min}$ ) ratio of ventilation to perfusion in the lungs

discrepancy between ventilation and blood flow in localised areas of the lung, causing decrease in oxygen level and rise in $\mathrm{CO}_{2}$ level 
Corrections

doi:10.1136/thx.2008.102947corr1

O'Driscoll B R, Howard L S, Davison A G on behalf of the British Thoracic Society. BTS guideline for emergency oxygen use in adult patients. Thorax 2008; 63(Suppl 6):vi1-vi68.

The Guideline Development Group regret that no advice was offered for the management of status epilepticus. As this is a life-threatening condition where a patient may suffer from cerebral hypoxia (and oximetry may not be possible), patients with status epilepticus should be treated in accordance with table 1 (reservoir mask until clinically stabilised). This advice also applies to other rare conditions that may cause life-threatening hypoxaemia that are not listed specifically in table 1.

Roberts JA. Benchmarking chronic obstructive pulmonary disease across an inner city primary care trust: one year on. Thorax 2008;63(Suppl vii):A8 (abstract S12).

The correct authors for this abstract are as follows: Roberts JA, Bakerly ND.

Roberts JA. Should chronic obstructive pulmonary disease service delivery in an inner city primary care trust be targeted at general practice or practice-based commissioning cluster level? Thorax 2008;63(Suppl vii):A8 (abstract S13).

The correct authors for this abstract are as follows: Roberts JA, Bakerly ND.

Tsartsali L, Fleming L, Regamey N, et al. Relationship between non-invasive inflammatory markers and the current level of clinical control in childhood asthma. Thorax 2008;63(Suppl vii):A33 (abstract S70).

This abstract has been withdrawn.

Baird S, Ashish A, O'Connor J, et al. Respiratory assessment centre: does it increase the number of patients taken home with the early supported discharge team? Thorax 2008;63(Suppl vii):A67 (abstract S154).

It has come to the attention of the Scientific Committee that this abstract was not seen by all the authors prior to submission and is therefore withdrawn.

doi:10.1136/thx.2007.088831 corr1

Creagh-Brown B C, Nicholson A G, Showkathali R, et al. Pulmonary veno-occlusive disease presenting with recurrent pulmonary oedema and the use of nitric oxide to predict response to sildenafil Thorax 2008;63:933-4.

The address for correspondence should be: Dr B C Creagh-Brown, Adult Intensive Care Unit, Royal Brompton Hospital, London SW3 6NP, UK; drbencb@gmail.com. 
Corrections

doi:10.1136/thx.2008.102947corr1

O'Driscoll B R, Howard L S, Davison A G on behalf of the British Thoracic Society. BTS guideline for emergency oxygen use in adult patients. Thorax 2008; 63(Suppl 6):vi1-vi68.

The Guideline Development Group regret that no advice was offered for the management of status epilepticus. As this is a life-threatening condition where a patient may suffer from cerebral hypoxia (and oximetry may not be possible), patients with status epilepticus should be treated in accordance with table 1 (reservoir mask until clinically stabilised). This advice also applies to other rare conditions that may cause life-threatening hypoxaemia that are not listed specifically in table 1.

Roberts JA. Benchmarking chronic obstructive pulmonary disease across an inner city primary care trust: one year on. Thorax 2008;63(Suppl vii):A8 (abstract S12).

The correct authors for this abstract are as follows: Roberts JA, Bakerly ND.

Roberts JA. Should chronic obstructive pulmonary disease service delivery in an inner city primary care trust be targeted at general practice or practice-based commissioning cluster level? Thorax 2008;63(Suppl vii):A8 (abstract S13).

The correct authors for this abstract are as follows: Roberts JA, Bakerly ND.

Tsartsali L, Fleming L, Regamey N, et al. Relationship between non-invasive inflammatory markers and the current level of clinical control in childhood asthma. Thorax 2008;63(Suppl vii):A33 (abstract S70).

This abstract has been withdrawn.

Baird S, Ashish A, O'Connor J, et al. Respiratory assessment centre: does it increase the number of patients taken home with the early supported discharge team? Thorax 2008;63(Suppl vii):A67 (abstract S154).

It has come to the attention of the Scientific Committee that this abstract was not seen by all the authors prior to submission and is therefore withdrawn.

doi:10.1136/thx.2007.088831 corr1

Creagh-Brown B C, Nicholson A G, Showkathali R, et al. Pulmonary veno-occlusive disease presenting with recurrent pulmonary oedema and the use of nitric oxide to predict response to sildenafil Thorax 2008;63:933-4.

The address for correspondence should be: Dr B C Creagh-Brown, Adult Intensive Care Unit, Royal Brompton Hospital, London SW3 6NP, UK; drbencb@gmail.com. 